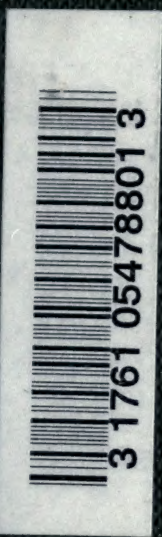





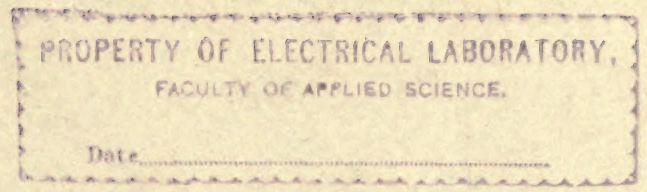


Digitized by the Internet Archive in 2007 with funding from Microsoft Corporation 


\section{ADVANCED \\ ELECTRICITY AND MAGNETISM}




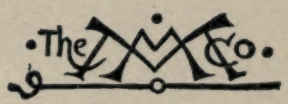

THE MACMILLAN COMPANY

NEW YORK BOSTON CHICAGO

DALLAS SAN FRANCISCO

MACMILLAN \& CO., Limited

LONDON BOMBAY CALCUTTCA

MELBOURNE

THE MACMILLAN CO. OF CANADA, LtD. TORONTO 


\title{
ADVANCED THEORY OF
}

\section{ELECTRICITY AND MAGNETISM}

\author{
A TEXT-BOOK FOR COLLEGES \\ AND TECHNICAL SCHOOLS
}

BY

WILLIAM S. FRANKLIN AND BARRY MACNUTT

Nelw York THE MACMILLAN COMPANY

London: Macmillan \& Co., LtD. I9I 5

All rights reserved 


\section{Copyright I9r5}

\section{By The Macmillan Company}

Set up and electrotyped. Published February, xgr5

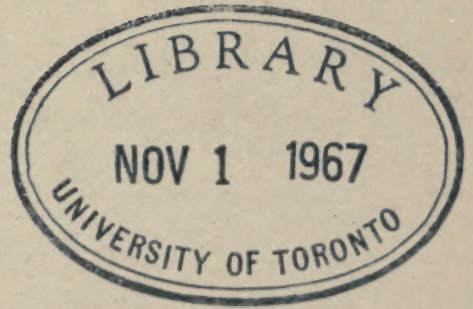




\section{PREFACE}

To make the study of theoretical physics something besides a purely formal mathematical exercise, it is necessary to keep physical things always clearly and vividly in mind. It is comparatively easy to do this in the study of applied science, but it is extremely difficult to do it in the study of many of the unfamiliar topics which are discussed in this book. The character of the treatment in this book has been determined throughout by the desire to keep the student's mind jammed up tight against physical things! The chapter on potential, for example, is kept within severely concrete limits, and the student who wishes to get an insight into the elementary mathematical theory of potential should read chapter IX of Franklin, MacNutt and Charles' Calculus, published by the authors, South Bethlehem, Pa., I9I3.

The authors desire to express their appreciation of the great assistance which Professor R. L. Charles has rendered in the preparation of this book.

JANUARY 16, 1915.

W. S. Frankín

BARRY MACNUTT. 



\section{TABLE OF CONTENTS}

\section{PART I}

Chapter I

Elementary Theory of Ferro-Magnetism . . . . . I-I7

Chapter II

Elementary Theory of Electro-Magnetism . . . . 18-36

Chapter III

Induced Electromotive Force and Inductance • • • 37-74

Chapter IV

The Magnetic Properties of Iron . . . . . . 75-103

Chapter V

Ship's Magnetism and the Compensation on the Compass . 104-120 PART II

Chapter VI

Electric Charge and the Condenser . . . . . . I2I-I36

Chapter VII

Theory of the Electric Field . . . . . . . . . I37-I64

Chapter ViII

Theory of Potential . . . . . . . . . . I65-I92

Chapter IX

Electric Oscillations and Electric Waves . • . . 193-273 PART III

Chapter $\mathrm{X}$

The Election Theory . . . . . . • . . • . 274-297

vii 



\section{ADVANCED \\ ELECTRICITY AND MAGNETISM.}

\section{CHAPTER I.}

ELEMENTARY THEORY OF MAGNETISM.

I. Ferromagnetism and electromagnetism.-There are two groups of magnetic phenomena, namely, $(a)$ the phenomena of ferromagnetism, that is to say, the phenomena which are associated with magnetized iron and steel, and $(b)$ the phenomena of electromagnetism, that is to say, the magnetic phenomena which are exhibited by the electric current. In developing the theory of magnetism it is best to consider some phases of ferromagnetism first, because the phenomena of ferromagnetism are more familiar than the phenomena of electromagnetism and because important magnetic measurements are based upon ferromagnetism.

The phenomena of electromagnetism are comparatively obscure, and in many cases imperceptible, except where they are enhanced by the presence of iron. Thus a dynamo or a transformer would operate if all iron parts were removed, but the effects produced would be in most cases nearly imperceptible. Practically, the phenomena of ferromagnetism and the phenomena of electromagnetism are inextricably mixed up with each other.

2. Poles of a magnet.-The familiar property of a magnet, namely, its attraction for iron, is possessed only by certain parts of the magnet. These parts of a magnet are called the poles of the magnet. For example, the poles of a straight bar-magnet are usually at the ends of the bar. 
When a bar-magnet is suspended in a horizontal position by a fine thread, it places itself approximately north and south like a compass needle. The north pointing end of the magnet is called its north pole, and the south pointing end of the magnet is called its south pole.

The north poles of two magnets repel each other, the south poles of two magnets repel each other, and the north pole of one magnet attracts the south pole of another magnet; that is to say, like magnetic poles repel each other, and unlike magnetic poles attract each other.

The mutual force action of two magnets is, in general, resolvable into four parts, namely, the forces with which the respective poles of one magnet attract or repel the respective poles of the other magnet. In the following discussion we consider only the force with which one pole of a magnet acts upon one pole of another magnet, not the forces with which one complete magnet acts on another complete magnet.

3. Distributed poles and concentrated poles.-The poles of a bar magnet are always distributed over considerable portions of the bar. This is especially the case with short thick bars. In the case of a long slim bar magnet, however, the poles are ordinarily approximately concentrated at the ends of the bar. The forces of attraction and repulsion of concentrated magnet

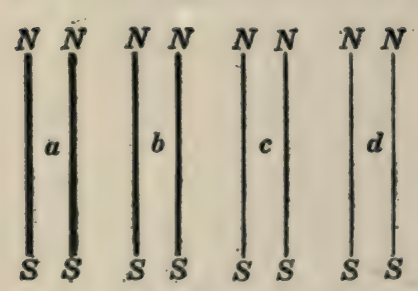

Fig. 1.

Pairs of exactly similar magnets. poles are easily formulated, therefore the following discussion applies to ideally concentrated poles at the ends of ideally slim bar magnets.

4. Definition of unit pole.-Consider a large number of pairs of magnets $a, b, c, d$, etc., as shown in Fig. I, the two magnets of each pair being exactly alike.* From such a set it would be possi-

* That is, the magnets of each pair are made of identically the same kind of steel, sebjected to the same kind of heat treatment and magnetized by the same means. 
ble to select a pair of magnets such that the north pole of one magnet would repel the north pole of the other with a force of one dyne when they (the two north poles) are one centimeter apart; each pole of such a pair is called a unit pole. That is, a unit pole is a pole which will exert a force of one dyne upon another unit pole at a distance of one centimeter.

5. Strength of pole.-Let us choose a slim magnet with unit poles, and let us use one of these poles as a "test pole." Any

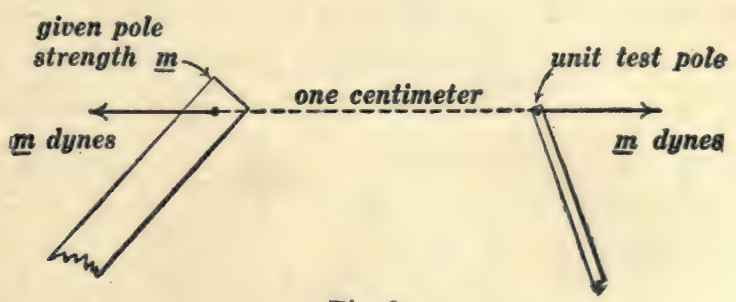

Fig. 2 .

given magnet pole is said to have more or less strength according as it exerts more or less force on our "test pole" at a given distance. And the force $m$ (in dynes) with which the given pole attracts or repels (or is attracted or repelled by) the unit test pole at a distance of one centimeter is taken as the measure of the strength of the given pole. That is, a given pole has $m$ units of strength when it will exert a force of $m$ dynes on a unit pole at a distance of one centimeter, as indicated in Fig. 2.

6. Attraction and repulsion of magnet poles.-Unlike poles attract and like poles repel each other, as stated in Art. 2. When the two attracting or repelling poles are unit poles their attraction or repulsion is equal to one dyne when they are one centimeter apart, and the attraction or repulsion of two poles whose respective strengths

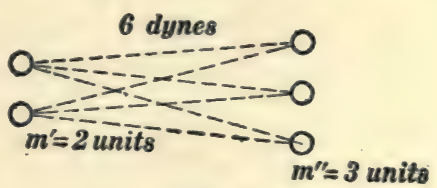

Fig. 3. are $m^{\prime}$ and $m^{\prime \prime}$ is equal to $m^{\prime} m^{\prime \prime}$ dynes when the poles are one centimeter apart. One may think of each unit of $m^{\prime}$ as exerting 
a force of one dyne on each unit of $m^{\prime \prime}$. Thus if $m^{\prime}=3$ units and $m^{\prime \prime}=2$ units, then the force of attraction or repulsion will be six dynes, as indicated in Fig. 3, where each dotted line represents one dyne.

7. Complete expression for the force of attraction or repulsion of two magnet poles.-Coulomb discovered in 1800 that the force of attraction or repulsion of two magnet poles is inversely proportional to the square of the distance between them (Coulomb's law). But the force of attraction or repulsion of two magnet poles when they are one centimeter apart is $m^{\prime} m^{\prime \prime}$ dynes as explained in Art. 6. Therefore, according to Coulomb's law, the force of attraction or repulsion is $\frac{m^{\prime} m^{\prime \prime}}{r^{2}}$ dynes when the poles are $r$ centimeters apart. That is:

$$
F=\frac{m^{\prime} m^{\prime \prime}}{r^{2}}
$$

in which $m^{\prime}$ and $m^{\prime \prime}$ are the respective strengths of two magnet poles, $r$ is their distance apart in centimeters, and $F$ is the force in dynes with which the poles attract or repel each other.

Algebraic sign of magnet pole. It is customary to consider a north pole as positive and a south pole as negative. That is, $m$ is positive when it expresses the strength of a north pole and negative when it expresses the strength of a south pole. Therefore, the product $m^{\prime} m^{\prime \prime}$ is positive when both poles are north poles or when both poles are south poles, and in this case the force $F$ in equation (I) is a repulsion. The product $m^{\prime} m^{\prime \prime}$ is negative when one pole is a north pole and the other pole is a south pole, and in this case the force $F$ in equation (I) is an attraction. Therefore, when $F$ in equation (I) is positive it is a repulsion, and when it is negative it is an attraction.

8. Direction and intensity of a magnetic field at a point.A magnetic field may be defined as a region in which a suspended magnetic needle tends to point in a definite direction, and the 
"north pole" of the needle points* in what is called the direction of the field. Thus the entire region surrounding the earth is a magnetic field; the region surrounding a magnet is a magnetic field; and the region surrounding a wire in which an electric current is flowing is a magnetic field. Figure 4 shows a compass needle placed at a point $p$ near a large magnet. The dotted arrow shows the direction of the magnetic field at $p$.

The poles of a magnet are acted upon by equal and oppo-

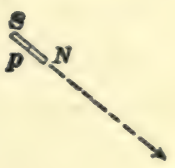

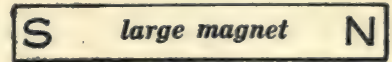

Fig. 4.

The dotted line shows the direction in which the small magnet at $p$ points.

site forces when the magnet is placed in a magnetic field. (This statement refers to what is called a uniform magnetic field, see Art. II.)

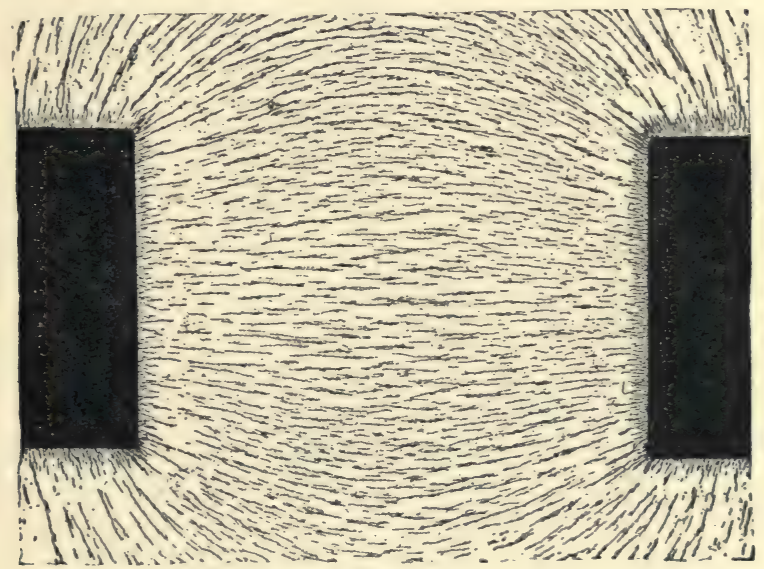

Fig. 5a.

Figure $5^{a}$ is a photograph of the figure obtained by dusting iron filings on a pane of glass which is placed over two large

* If the needle is perfectly balanced. 
magnet poles, a north pole and a south pole, facing each other. The filaments of iron filings show what are called the lines of force of the magnetic field; a line of force being a line drawn so as to be at each point in the direction of the field at that point. The magnetic field in the central region in Fig. $5 a$ is approximately uniform.

Figure $5^{b}$ represents a small magnet held in the approximately uniform magnetic field between two large magnet poles, and the arrows represent the equal and opposite forces which are exerted on the small magnet by the approximately uniform field.
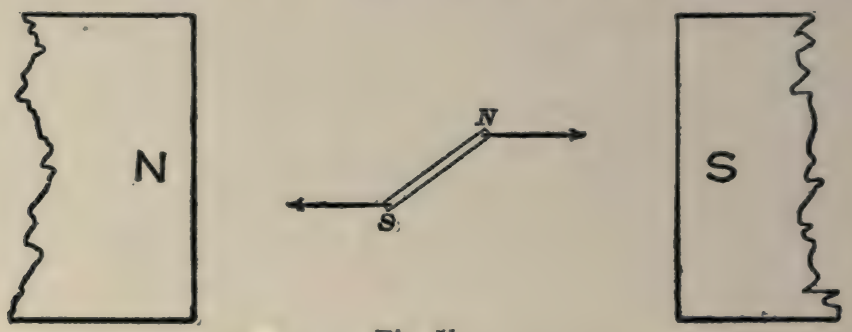

Fig. $5 b$.

The arrows show the forces which act upon the poles of the small magnet.

The force $H$ (in dynes) which a magnetic field exerts upon a unit test pole is used as a measure of the intensity or strength of the magnetic field, and this force-per-unit-pole is hereafter spoken of simply as the intensity or strength of the field. The unit of magnetic field intensity (one dyne per unit pole) is called a gauss. That is, a magnetic field has an intensity of one gauss when it will exert a force of one dyne upon a unit pole.

Complete expression for the force exerted on a magnet pole by a magnetic field.-A magnetic field of which the intensity is $H$ gausses exerts a force of $H$ dynes upon a unit pole as above explained, and it exerts a force of $m H$ dynes upon a pole of which the strength is $m$ units. That is:

$$
F=m H
$$

in which $F$ is the force in dynes which is exerted on a pole of strength $m$ by a field of intensity $H$. 
Uniform and non-uniform fields.-A magnetic field is said to be uniform when it has everywhere the same direction and the same intensity, otherwise the field is said to be non-uniform. The earth's magnetic field is sensibly uniform throughout a room. The magnetic field surrounding a magnet is non-uniform. The magnetic field surrounding an electric wire is non-uniform.

9. Direction and intensity of the magnetic field surrounding an "isolated " magnet pole of strength $M$.-By an "isolated" magnet pole is meant one pole of a very long slim magnet-the other pole being so far away as to be negligible in its action.

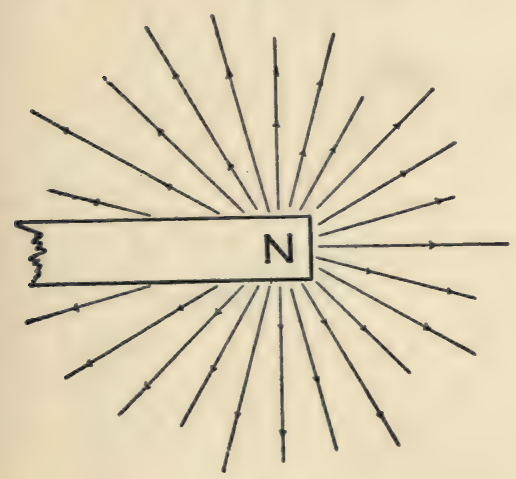

Fig. 6.

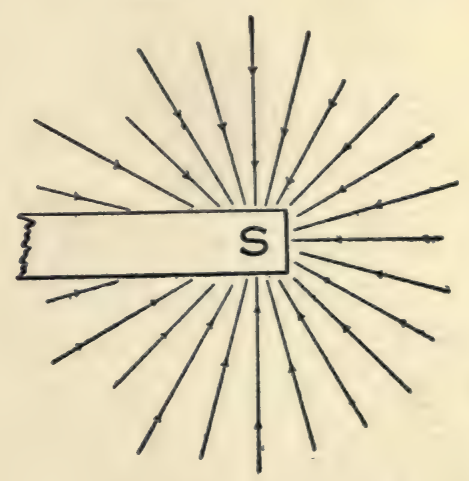

Fig. 7.

The magnetic field in the neighborhood of an isolated north pole is everywhere directed away from the pole as shown by the radiating straight lines (lines of force, as they are called) in Fig. 6. The magnetic field in the neighborhood of an isolated south pole is everywhere directed towards the pole as indicated in Fig. 7.

Consider two magnet poles $M$ and $m$ which are at a distance of $r$ centimeters apart as shown in Fig. 8. The force $F$ with which $M$ repels $m$ is equal to $\frac{M m}{r^{2}}$, according to Art. 7 ; but the force exerted on $m$ can also be expressed as equal to $m H$ where $H$ is the intensity at $m$ of the magnetic field which is due 
to $M$. Therefore $m H=\frac{M m}{r^{2}}$, whence we have:

$$
H=\frac{M}{r^{2}}
$$

in which $H$ is the intensity of the magnetic field produced by the pole $M$ at a place which is $r$ centimeters from $M$.

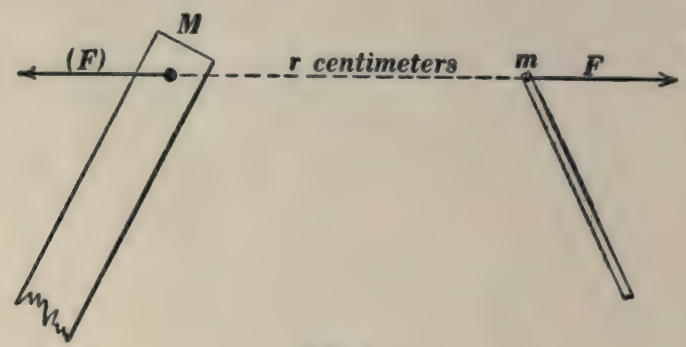

Fig. 8.

10. Action of a magnetic field on iron or steel.-When an iron or steel rod is placed in a magnetic field, the length of the rod being parallel to the direction of the field, the rod becomes a magnet. Thus the iron rod $A B$ in Fig. 9 is magnetized by the field due to the large magnet pole $N$, the end $A$ becomes a south pole and the end $B$ becomes a north pole.
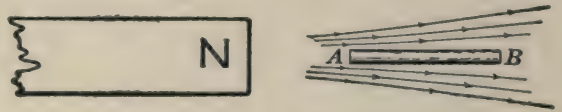

Fig. 9.

The effect of the magnetic field on the iron $\operatorname{rod} A B$ is to magnetize it, the end $A$ becoming a south pole.

II. Behavior of a magnet in a magnetic field, (a) Behavior in a uniform field.-The equal and opposite forces which are exerted on the poles of a magnet by a uniform magnetic field tend only to turn the magnet into the direction of the field, the forces do not tend to produce translatory motion of the magnet. Consider a magnet $l$ centimeters long placed in a uniform magnetic field of which the intensity is $H$, the angle between the axis of the 
magnet and the direction of the field being $\theta$ as shown in Fig. Io. By length of magnet is meant the distance between its poles. The poles of the magnet are acted upon by the two opposite forces

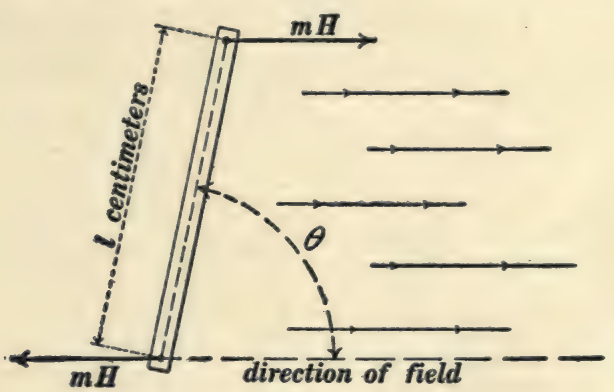

Fig. 10.

The equal and opposite forces which act upon the poles of the magnet constitute a torque which tends to turn the magnet into the direction of the field.

$m H$ as shown, and the combined torque action of these forces about an axis perpendicular to the plane of the paper is $m l H$ $\sin \theta$. That is:

$$
T=-m l H \sin \theta
$$

where $T$ is the torque action of the forces $m H$ in Fig. Io. The negative sign is chosen because the torque $T$ tends to reduce $\theta$ which may be considered to be a positive angle. This equation expresses $T$ in dyne-centimeters. If the angle $\theta$ is always very small then the value of $\theta$ in radians is sensibly equal to $\sin \theta$, and in this case equation (I) becomes:

$$
T=-m l H \cdot \theta
$$

This equation shows* that a suspended magnet when started will perform harmonic vibrations about its axis of suspension such that:

$$
\frac{4 \pi^{2} K}{t^{2}}=m l H
$$

in which $K$ is the moment of inertia of the steel bar (the magnet) about the axis of suspension, and $t$ is the period of one complete

* See Arts. 42 and 66, Franklin and MacNutt's Mechanics and Heat. 
vibration. This equation is true only when the amplitude of the vibrations is very small, that is, when the angle $\theta$ never becomes large.

(b) Behavior in a non-uniform field.-The forces which are exerted upon the poles of a magnet in a non-uniform magnetic

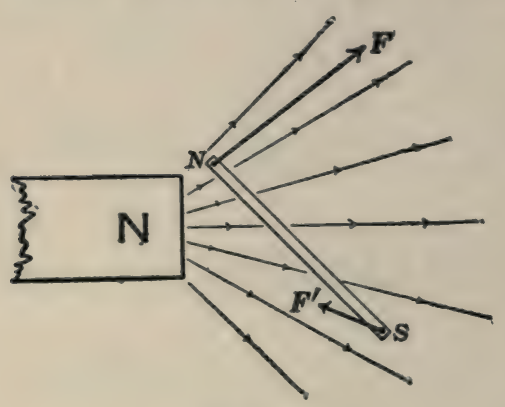

Fig. 11.

Showing the unequal forces with which a non-uniform field acts upon the poles of a magnet. field are, in general, not equal in value and not opposite in direction, and therefore such forces tend not only to turn the magnet but also to impart to it a motion of translation. Thus Fig. II shows the two forces $F$ and $F^{\prime}$ which are exerted upon the poles of a small magnet by a non-uniform magnetic field. The forces $F$ and $F^{\prime}$ are not the same in value and not opposite in direction.

In order that a particle of iron may be attracted by a magnet it is necessary for the particle of iron to be magnetized, and also it is necessary for the magnetized particle to be in a non-uniform magnetic field. If the particle of iron is in a uniform field, equal and opposite forces are exerted upon its two poles, and it tends only to turn and point in a certain direction.

The magnetic field near a flat ended magnet pole is approximately uniform (lines of force parallel straight lines) as shown in Fig. 12a. Near the sharp corners of the pole, however, the field is distinctly non-uniform (lines of force diverge strongly). Therefore particles of iron are not appreciably attracted by the flat end of the pole, whereas the sharp corners of the pole attract particles of iron very strongly. This is strikingly shown by passing a flat ended magnet pole over a table on which a very few iron filings have been placed. The filings are all caught by the corners of the pole.

The lines of force in the neighborhood of a sharp pointed 
magnet pole diverge very greatly indeed as shown in Fig. I2b, that is to say, the magnetic field in the neighborhood of a pointed pole is non-uniform to a high degree, and such a magnet pole has a strong attraction for small particles of magnetic material. Surgeons make use of a pointed magnet in removing particles of

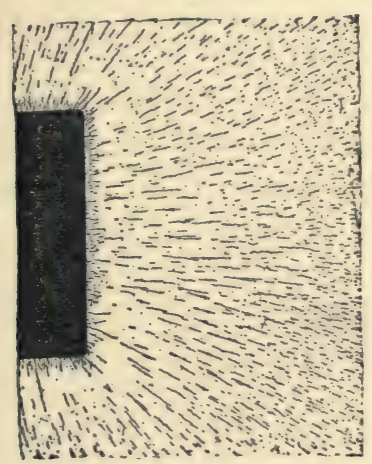

Fig. 12a.

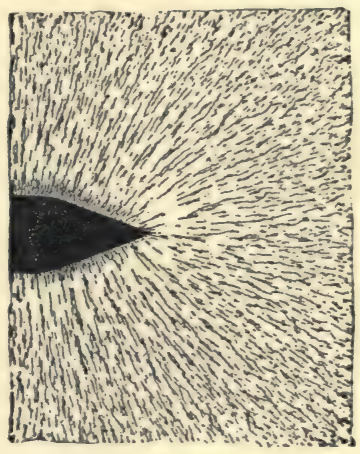

Fig. $12 b$.

iron or steel from the eye. If it is desired to separate magnetic particles from non-magnetic particles in a finely crushed ore, a pointed magnet pole must be used to attract the magnetic particles.

\section{I2. Gauss's method for measuring the horizontal com-} ponent $H^{\prime}$ of the earth's magnetic field.-From about 1860 to I880 the measurement of the intensity of the horizontal component of the earth's magnetic field, $H^{\prime}$, by the method of Gauss was of fundamental importance, because the tangent galvanometer was then extensively used for measuring electric current, and to measure an electric current by the tangent galvanometer, the value of $H^{\prime}$ must be known. The tangent galvanometer has, however, been superseded by the electrodynamometer (see description of Weber's form of electrodynamometer in Art. 22) for the fundamental measurement of electric current, and therefore Gauss's method is now important only in the making of magnetic surveys. The method is however of very 
great interest in that it brings the abstract discussion of Arts. 4 to 9 into relation with actual experiment.

Gauss's method involves two independent sets of observations with two different arrangements of apparatus, as follows:

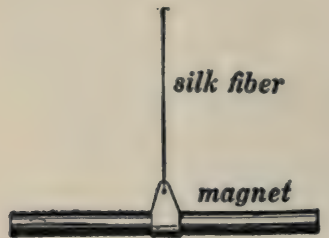

Fig. 13.

First arrangement.-A bar magnet is suspended, as shown in Fig. I3, at the place where the value of $H^{\prime}$ is to be determined, and set vibrating through a small amplitude about the suspending fiber as an axis; and the time $t$ of one complete vibration is observed. Then from equation (3) of Art. I I we have:

$$
\frac{4 \pi^{2} K}{t^{2}}=m l H^{\prime}
$$

The moment of inertia $K$ of the bar magnet is known, being determined from the measured dimensions and mass (in grams) of the bar. Therefore the quantities $m, l^{*}$ and $H^{\prime}$, only, are unknown.

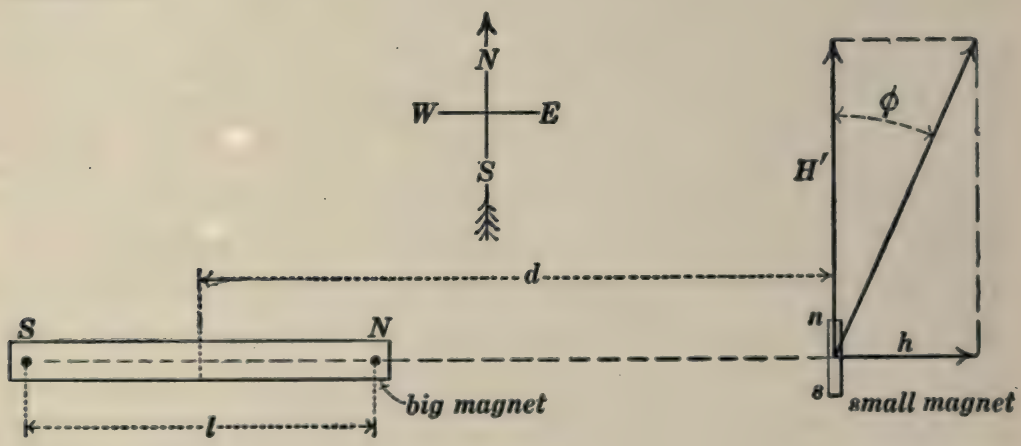

top view.

Fig. 14.

Second arrangement.-A very small magnet, ns, Fig. 14, is suspended by a fine silk fiber at the place occupied by the large bar magnet in the first arrangement. This small magnet $n s$,

* Of course $l$ is somewhat less than the length of the bar. 
being free to turn, points in the direction of the magnetic field in which it is placed, that is, in the direction of $H^{\prime}$.

The large magnet (the one used in the first arrangement) is now placed at a measured distance $d$ due magnetic east or west of $n s$, and the small magnet $n s$ turns through the angle $\phi$ and points in the direction of the resultant of $H^{\prime}$ and $h$, where $h$ is the intensity at $n s$ of the magnetic field due to both poles of the large bar magnet. The angle $\phi$ is observed, and we have:

$$
\tan \phi=\frac{\frac{m}{\left(d-\frac{1}{2} l\right)^{2}}-\frac{m}{\left(d+\frac{1}{2} l\right)^{2}}}{H^{\prime}}
$$

in which the only unknowns are $m, l$ and $H^{\prime}$.

The large bar magnet is now placed at a measured distance $d^{\prime}$ due magnetic east or west of $n s$, and the angle $\phi^{\prime}$ (corresponding to the angle $\phi$ in Fig. 14) is observed. Then we have

$$
\tan \phi^{\prime}=\frac{\frac{m}{\left(d^{\prime}-\frac{1}{2} l\right)^{2}}-\frac{m}{\left(d^{\prime}+\frac{1}{2} l\right)^{2}}}{H^{\prime}}
$$

in which the only unknowns are $m, l$ and $H^{\prime}$.

The three unknown quantities can be calculated with the help of equations (I), (2) and (3).

Derivation of equation (2).-The intensity $h$ of the magnetic field at $n s$ due to the big magnet in Fig. I4 is the algebraic sum of the field intensities at $n s$ due to the two poles of the big magnet. The field intensity at $n s$ due to the north pole of the big magnet is $\frac{m}{\left(d-\frac{1}{2} l\right)^{2}}$ according to Art. 9, and this field is to the right in Fig. I4. Similarly, the field intensity at $n s$ due to the south pole is $\frac{m}{\left(d+\frac{1}{2} l\right)^{2}}$ to the left in Fig. I4. Therefore $h$ in Fig. 14 is:

$$
h=\frac{m}{\left(d-\frac{1}{2} l\right)^{2}}-\frac{m}{\left(d+\frac{1}{2} l\right)^{2}}
$$


where $m$ is the strength of each of the poles of the big magnet. Furthermore, we have from Fig. I4:

$$
\tan \phi=\frac{h}{H^{\prime}}
$$

Therefore, using the above expression for $h$ we get equation (2).

\section{PROBLEMS.}

I. Two permanent magnets I centimeter $\times 0.5$ centimeter $\times 30$ centimeters long are magnetized to an intensity of 700 units pole per square centimeter of sectional area. (a) Calculate the strength of each pole. (b) Calculate the force with which the north pole of one rod attracts the south pole of the other rod when the poles are at an approximate distance of ro centimeters from each other. Ans. (a) 350 units pole. (b) 1,225 dynes.

Note.-In this and the following problems, assume the poles of the magnet to be concentrated at the center of the ends of the bars. The intensity of magnetization of an iron rod is the strength of pole on one end divided by the sectional area of the rod.

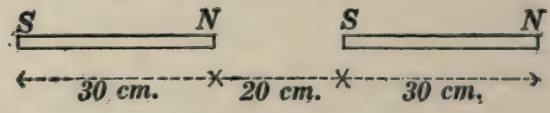

Fig. $p 2$.

2. The two magnets specified in problem $\mathrm{I}$ are arranged as shown in Fig. p2. Find the total force with which one magnet acts upon the other magnet. Ans. 227.39 dynes attraction.

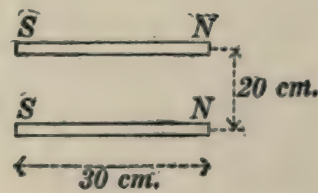

Fig. p3.

3. The two magnets specified in problem $\mathrm{I}$ are arranged as shown in Fig. p3. Find the total force with which one magnet acts on the other magnet. Ans. 507.8 dynes repulsion. 
4. The two magnets specified in problem I are arranged as shown in Fig. p4. Find the total force with which one magnet acts on the other. Ans. 507.8 dynes attraction.

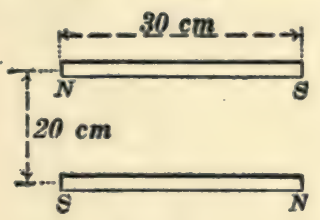

Fig. $p 4$.

5. A magnet I .o by 0.25 by 40 centimeters long, having 800 units pole per square centimeter of sectional area, is laid across one of the magnets specified in problem $\mathrm{I}$, as shown in Fig. p5. Find

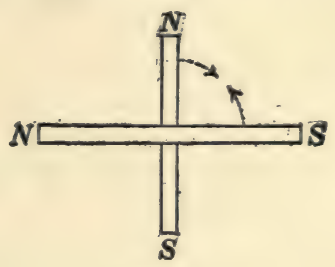

Fig. $p 5$.

the total force with which one magnet acts on the other. Ans. 5,376 dyne-centimeters of torque tending to turn magnets as shown by arrows in Fig. p5.

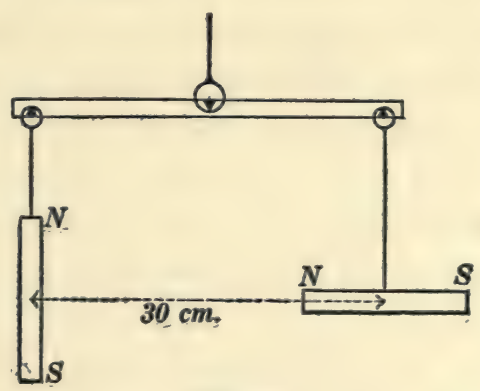

Fig. $p 6$.

6. The two magnets specified in problem I are hung from a balance beam as indicated in Fig. p6. Assuming that the 
magnets exactly balance each other before they are magnetized, find the number of grams which must be added to one pan to balance the magnets after they are magnetized, and specify to which pan the weight must be added. Ans. 0.7I5 gram must be added to the left pan.

7. Determine the intensity $H$ of the magnetic field at a point $p$ distant 18 centimeters from one pole and 24 centimeters from the other pole of one of the magnets specified in problem $\mathbf{I}$, and

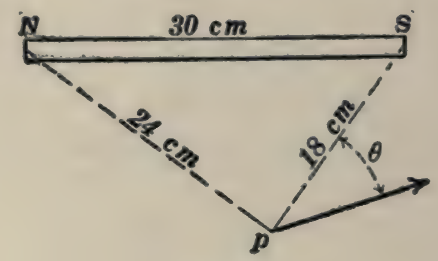

Fig. $p 7$.

determine the value of the angle $\theta$, as shown in Fig. p7. Ans. $H=\mathrm{I} .24$ gausses, $\theta=66^{\circ} \mathrm{I} 3^{\prime} \cdot 5$.

Note.-When two causes are acting together to produce a magnetic field, the field which is produced by both causes together is represented by the diagonal of the parallelogram whose sides represent the field intensities at the point due to the respective causes separately.

8. The horizontal component of the earth's magnetic field at a given place is 0.18 gauss, and its direction is due north. It is desired to produce at the given place a resultant magnetic field of 0.02 gauss intensity in a due easterly direction. Find the distance and direction from the place to the point at which an isolated north magnet pole of 600 units strength must be placed to produce the desired result. Ans. $57.6 \mathrm{~cm}$., $6^{\circ} 20^{\prime}$ west of north.

9. The intensity of the earth's magnetic field at Washington is $0.5^{8}$ gauss and its dip is $62^{\circ}$. Find its horizontal and vertical components. Ans. Horizontal component $=0.272$ gauss; vertical component $=0.512$ gauss.

Io. Find the direction and intensity of the resultant magnetic field at a point 30 centimeters due magnetic north of an isolated 
north pole of 600 units strength at Washington. Ans. 1.07 gausses, north, and dipping $28^{\circ} 36^{\prime}$ below the horizontal.

II. One of the magnets specified in problem I is balanced horizontally on a knife edge at Washington. The magnet weighs I20 grams. Find the horizontal distance from the knife edge to the center of the bar. Use the data specified in Problem 9. Ans. 0.046 centimeter.

12. The moment of inertia of one of the magnets specified in problem I is $9,000 \mathrm{gr} .-\mathrm{cm} .{ }^{2}$. Calculate the time of one complete oscillation of this magnet when it is suspended horizontally at Washington. Ans. II.I5 seconds.

I3. A magnet makes one complete oscillation per second in a magnetic field of which the intensity is 0.2 gauss. Another magnet is twice as long, twice as wide, and twice as thick, it is magnetized to twice the intensity (units pole per unit sectional area) and it is suspended in a field of which the intensity is o.I gauss. What is its period of oscillation? Ans. 2 seconds.

Note. - The moment of inertia of a rotating body is equal to the product of the mass of the body into the square of its radius of gyration. Given two bodies of exactly the same shape, their radii of gyration are proportional to their linear dimensions, whereas their masses are proportional to their volumes.

I4. A suspended magnet makes 20 oscillations in 184.5 seconds at one place, and 20 oscillations in 215.8 seconds at another place. What is the ratio of the intensities of the horizontal component of the earth's magnetic field at the two places, and at which place is it the more intense? Ans. I.367. Field more intense at first place. 


\section{CHAPTER II.}

\section{ELEMENTARY THEORY OF ELECTROMAGNETISM. THE MAGNETIC MEASUREMENT OF CURRENT.}

I3. Strength of electric current magnetically defined.-Consider a straight electric wire stretched across a uniform magnetic field, the wire being at right angles to the field as shown in Fig. I5. Let us suppose, for a moment, that the field is of unit

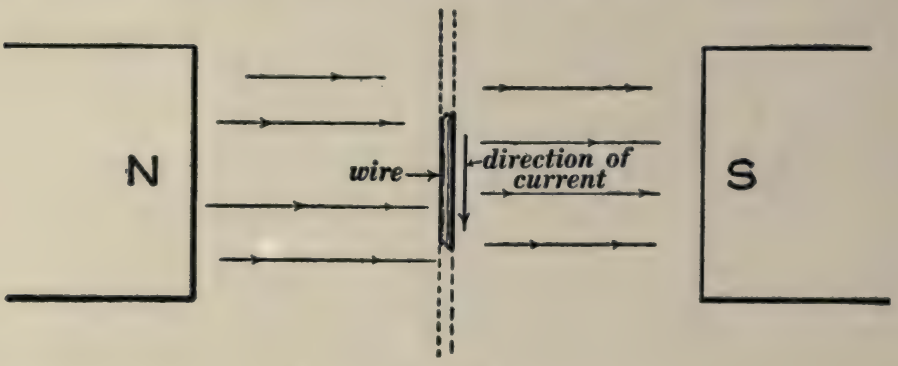

Fig. 15.

intensity. The force in dynes with which this unit field pushes sidewise on one centimeter of the electric wire has been adopted as the fundamental measure of the strength of the current in the wire. This force-per-unit-length-of-wire-per-unit-field-intensity is called simply the strength of the current in the wire, and it is represented by the letter $I$. The force pushing sidewise on $l$ centimeters of the wire is $l I$ dynes; and if the field intensity is $H$ gausses instead of one gauss, then the force is $H$ times as great, or $l I H$ dynes. That is

$$
F=l I H
$$

in which $F$ is the force in dynes pushing sidewise on $l$ centimeters of wire at right angles to a uniform magnetic field of 
which the intensity is $H$ gausses, and $I$ is the strength of the current in the wire.

Definition of the abampere.A wire is said to carry a current of one abampere when one centimeter of the wire is pushed sidewise with a force of one dyne, when the wire is stretched across a magnetic field of which the intensity is one gauss, the wire being at right angles to the field. The current $I$ in equation ( $I$ ) is expressed in abamperes when $F$ is expressed in dynes, $l$ in centimeters and $H$ in gausses.

\section{Definition of the ampere.-} The ampere is defined as one tenth of an abampere.

The c.g.s. system of electrical units.-In earlier days, the resistance of a particular piece of wire would be used as a unit of resistance, the electromotive force of a particular voltaic cell would be used as a unit of electromotive force, and current values were often specified in terms of the deflections of a particular galvanometer. The introduction of a uniform system of units was a great improvement on this old procedure, and it was brought about chiefly by Weber and Gauss in Germany and by Maxwell and Kelvin in England. This uniform system of units was based on the units already in use in mechanics, namely, the centimeter, the gram and the second; and the units of this c.g.s. system were called absolute units to distinguish them from the units formerly used.

The electrical units now almost universally employed, namely, the ampere, the volt, the ohm, the coulomb, the farad, and so forth, are not the c.g.s. units but convenient multiples or submultiples of them. The c.g.s. units as a rule have no names, therefore it is convenient to call the c.g.s. unit of current the 
abampere, the c.g.s. unit of resistance the abohm, the c.g.s. unit of electromotive force the abvolt, the c.g.s. unit of capacity the abfarad, and so forth.

The c.g.s. units here referred to are the so-called "electromagnetic" c.g.s. units. The c.g.s. units of the "electrostatic system" are entirely ignored in this text.

Definition of the abohm.A wire has a resistance of one abohm when one erg of heat is generated in it in one second by a current of one abampere.

Definition of the abvolt.An electric generator has an electromotive force of one abvolt when it delivers one erg per second of power with a current output of one abampere.

The abvolt may be defined on the basis of Ohm's law as the electromotive forcebetween the terminals of a resistance of one abohm when a current of one abampere is flowing through it.
Definition of the ohm.-A wire has a resistance of one ohm when one joule of heat is generated in it in one second by a current of one ampere. The $\mathrm{ohm}$ is equal to $\mathrm{IO}^{9}$ abohms.

\section{Definition of the volt.-An} electric generator has an electromotive force of one volt when it delivers one joule per second (one watt) of power with a current output of one ampere. The volt is equal to ${ }_{10}^{8}$ abvolts.

The volt may be defined on the basis of Ohm's law as the electromotive force between the terminals of a resistance of one ohm when a current of one ampere is flowing through it.

\section{The intensity of the magnetic field at the center of a} circular coil of wire.-If we can calculate the force with which a current in a circular coil of wire acts on a magnet pole of given strength placed at the center of the circular coil, we can derive an expression for the intensity of the magnetic field at the center of the coil due to the current in the coil, because the force exerted 
on the magnet pole by the coil of wire must be equal to $m h$ where $m$ is the strength of the pole and $h$ is the intensity at the pole of the field due to the coil.

Consider, therefore, a magnet pole of strength $m$ placed at the center of the circular coil as shown in Fig. I6. This pole produces a magnetic field of which the intensity at the wire is $\frac{m}{r^{2}}$, according to Art. 9, and the lines of force of this field are at right angles to the wire. Therefore, according to Art. I3, the wire is pushed sidewise (towards the reader in Fig. 16) with a force of $2 \pi r Z$ $\times I \times \frac{m}{r^{2}}$ dynes where $2 \pi r Z$ is the length of the wire ( $Z$ being the number of turns of

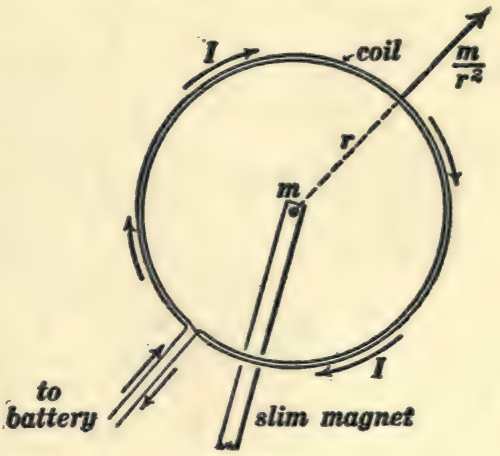

Fig. 16.

Circular coil of two turns with current $I$ flowing in it and with magnet pole $m$ at its center.

wire in the coil), and $I$ is the strength of the current in the coil in abamperes.

Now the force exerted on the coil by $m$ is equal and opposite to the force exerted on $m$ by the coil. Therefore, disregarding signs, we have

$$
m h=2 \pi r Z \times I \times \frac{m}{r^{2}}
$$

or

$$
h=\frac{2 \pi Z I}{r}
$$

where $h$ is the intensity at the center of the coil of the magnetic field due to a current of $I$ abamperes in the coil, $r$ is the radius of the coil in centimeters, and $Z$ is the number of turns of wire in the coil. 
I5. The tangent galvanometer.*-The tangent galvanometer consists essentially of a circular coil of wire at the center of which

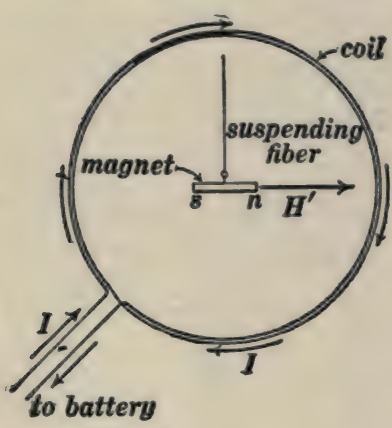

Fig. 17.

Circular coil of two turns with small magnet suspended at its center.

a small magnet is suspended as shown in Fig. 17. The suspended magnet carries a pointer which plays over a divided circle by means of which one may observe the angle $\phi$ through which the suspended magnet is turned when a current $I$ is sent through the coil. The coil is mounted with its plane vertical and magnetic north and south as may be seen from Fig. I8.

When no current is flowing through the coil the suspended magnet $n s$ points in the direction of $H^{\prime}$ (the horizontal component of the earth's magnetic field). When a current of $I$

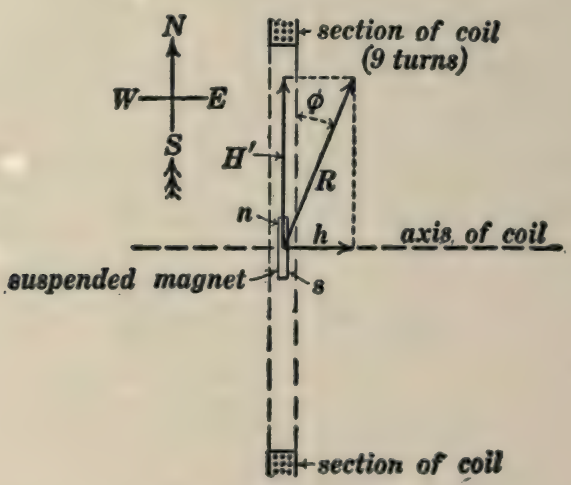

Fig. 18.

The plane of the paper is a horizontal plane.

abamperes flows through the coil the field $h\left(=\frac{2 \pi Z I}{r}\right.$ according to Art. I4) is produced, and the suspended magnet points in the

\footnotetext{
* See statement concerning the tangent galvanometer in Art. I2.
} 
direction of the resultant field $R$. Now $\tan \phi=\frac{h}{H^{\prime}}$, from Fig. 18. Therefore, using $\frac{2 \pi Z I}{r}$ for $h$ and solving for $I$, we have:

$$
I \text { in abamperes }=\frac{r H^{\prime}}{2 \pi Z} \cdot \tan \phi
$$

or

$$
I \text { in amperes }=\frac{5^{r H^{\prime}}}{\pi Z} \cdot \tan \phi
$$

I6. Intensity of the magnetic field at any point in the axis of a circular coil.-Consider the point $m$, Fig. I9, in the axis of a

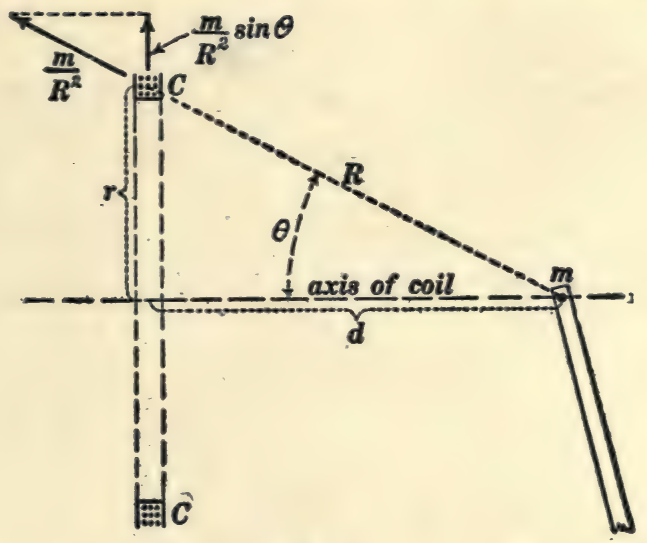

Fig. 19.

circular coil $C C$ of radius $r$ centimeters; the distance of $m$ from the plane of the coil being $d$ centimeters. Imagine a magnet pole of strength $m$ to be placed at $m$. This pole produces at $C$ a magnetic field of which the intensity is $\frac{m}{R^{2}}$ $\left(=\frac{m}{r^{2}+d^{2}}\right)$. The component of this field which is parallel to the axis of the coil pushes radially outwards on each part of the coil, tending only to spread the coil. But the component of $\frac{m}{R^{2}}$ which lies in the plane of the coil, namely, 


$$
\frac{m}{R^{2}} \cdot \sin \theta\left(=\frac{m}{r^{2}+d^{2}} \cdot \frac{r}{\sqrt{r^{2}+d^{2}}}=\frac{m r}{\left(r^{2}+d^{2}\right)^{3 / 2}}\right)
$$

pushes sidewise on the coil (to the right or left in Fig. 19), and the total force $F$ with which the magnet pole at $m$ pushes to the right or left on the coil in Fig. I9 is equal to the product of three factors, namely $(a)$ the length of wire in the coil which is $2 \pi r Z$, (b) the strength of the current in the coil in abamperes, and $(c)$ the radial component, $\frac{m}{R^{2}} \cdot \sin \theta$, of the magnetic field at $C$ due to $m$. Therefore:

$$
F=\frac{2 \pi r^{2} Z I m}{\left(r^{2}+d^{2}\right)^{3 / 2}}
$$

But the force with which the pole pushes on the coil is equal and opposite to the force with which the coil acts on the pole, and the force with which the coil acts on the pole may be expressed as $m h$, where $h$ is the intensity at the pole of the magnetic field due to the coil. Therefore, ignoring algebraic signs, we have:

or

$$
m h=\frac{2 \pi r^{2} Z I m}{\left(r^{2}+d^{2}\right)^{3 / 2}}
$$

$$
h=\frac{2 \pi r^{2} Z I}{\left(r^{2}+d^{2}\right)^{3 / 2}}
$$

in which $h$ is the intensity at the point $m$ in Fig. 19 of the magnetic field due to a current of $I$ abamperes in the circular coil $C C, Z$ is the number of turns of wire in the coil, $r$ is the radius of the coil, and $d$ is the distance of the point $m$ from the plane of the coil in centimeters.

I7. Magnetic field intensity inside of a very long coil.-It is desired to find the intensity at the point p, Fig. 20, of the magnetic field due to a very long cylindrical coil having $z$ turns of wire per centimeter of length, the current in the coil being $I$ abamperes. Let $\Delta H$ be the field intensity at $p$ due to the element $c c$ of the coil, $z \cdot d x$ being the number of turns of wire 
in the element $c c$. Then, according to equation (3) of Art. I6, we have:

$$
\Delta H=\frac{2 \pi r^{2} I z \cdot d x}{\left(r^{2}+x^{2}\right)^{3 / 2}}
$$

Every element of the long coil produces at $p$ a field which is in the same direction (parallel to the axis of the coil), and therefore the field at $p$ due to the entire coil is found by integrating ( $\mathrm{r}$ ) between the limits $x=-B$ to $x=+A$. For a very long coil the limits of the integration are from $x=-\infty$ to $x=+\infty$, and in this case we get:

$$
H=4 \pi z I
$$

where $H$ is the strength of the magnetic field inside of a very long coil having $z$ turns of wire per unit of length, and $I$ is the current in the coil in abamperes.

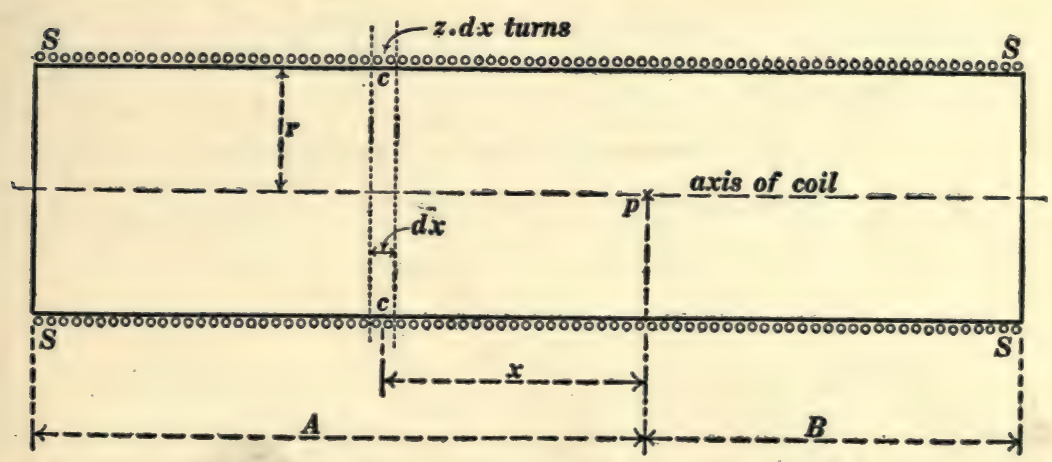

Fig. 20.

According to the above derivation, equation (2) gives the intensity of the magnetic field along the axis of a very long cylindrical coil, but as a matter of fact, the magnetic field inside of the coil is uniform, that is to say, it has everywhere the same intensity and it is everywhere in the same direction.

I8. Contribution to the magnetic field at a given point by one element of an electric wire.-The region surrounding an electric circuit is a magnetic field, and each element of the circuit (each 
element of the wire which constitutes the circuit) may be thought of as contributing its share to the field intensity at each point. In fact we have:

$$
\Delta H=\frac{I \sin \theta \cdot \Delta l}{r^{2}}
$$

in which $\Delta H$ is the field intensity at $m$ in Fig. $2 \mathrm{I}$ due to the short piece of wire $\Delta l$ which is a part of a circuit in which a current of $I$ abamperes is flowing, $r$ is the distance of $m$ from $\Delta l$, and $\theta$ is the angle shown in the figure; $r$ and $\Delta l$ are, of course, expressed in centimeters.

Imagine a magnet pole of strength $m$ placed at the point $m$ in Fig. 2I. If we can find an expression for the force exerted on

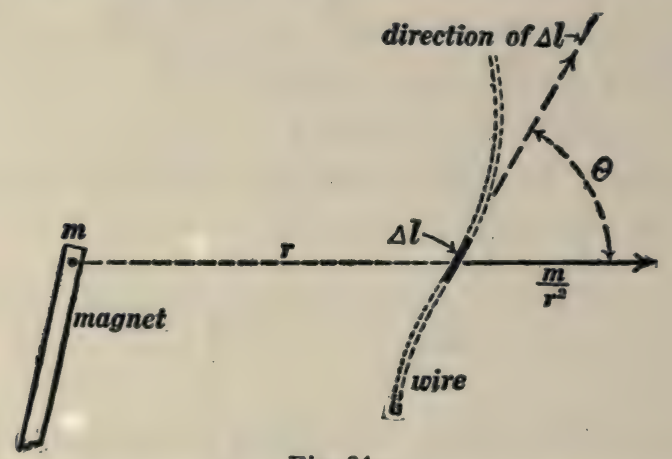

Fig. 21.

The field at $m$ due to $\Delta l$ is at right angles to the plane of the paper.

$\Delta l$ by the pole $m$, we will have an expression for the force exerted on the pole by $\Delta l$; but the force exerted on $m$ by $\Delta l$ is equal to the product of $m \cdot \Delta H$, where $\Delta H$ is the field intensity at $m$ due to $\Delta l$.

The field intensity at $\Delta l$ due to $m$ is $\frac{m}{r^{2}}$. The component of this field at right angles to $\Delta l$ is $\frac{m}{r^{2}} \sin \theta$, and this component pushes sidewise on $\Delta l$ with a force equal to $\Delta l \times I \times \frac{m}{r^{2}} \cdot \sin \theta$ according to equation (I) of Art. 13 ; and the force with which 
$\Delta l$ acts on $m$ is equal and opposite* to this. Therefore, ignoring algebraic signs, we have:

$$
m \cdot \Delta H=\Delta l \times I \times \frac{m}{r^{2}} \cdot \sin \theta
$$

from which equation (I) follows at once.

Proposition.-The intensity of the magnetic field at a given point in the neighborhood of a given coil of wire is proportional to the strength of the current in the coil, and the direction of the field at that point is fixed. That is to say, if the strength of the current is doubled, the intensity of the field will be everywhere doubled, but the direction of the field will be everywhere the same as before. The trend of the lines of force of the magnetic field due to a coil or circuit depends only on the shape and size of the coil or circuit, not at all on the strength of the current. $\dagger$

I9. Magnetic field due to a long straight wire.-The lines of force of the magnetic field surrounding a long straight electric wire are circles with their planes at right angles to the wire and

* A curious absurdity is involved here. Figure 22 shows an edgewise view of Fig. 21; $F$ is the force with which $m$ acts on $\Delta l$, and $F^{\prime}$ is the equal and opposite force with which $\Delta l$ acts on $m$; and these forces do not have the same line of action. This absurdity is due to the non-physical character of an element

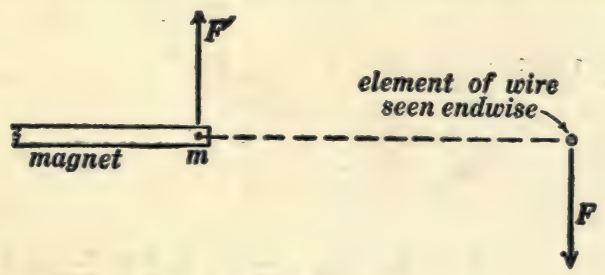

Fig. 22.

$\Delta l$ of an electric circuit. In so far as magnetic effect is concerned, an electric circuit is always complete. Thus the increasing electrical stress in the dielectric of a condenser which is being charged is equivalent magnetically to a flow of current through the dielectric of the condenser.

The impossible consequences of the physical absurdity in equation (I) of Art. I8 always disappear when the equation is integrated around a complete circuit.

$\dagger$ This proposition may be established by an argument based upon equation (I) above, the essential point being that when equation $(I)$ is integrated the constant factor $I$ can be taken from under the integral sign. 
with their centers on the axis of the wire. The intensity of the field at the point $m$ in Fig. 23 can be determined by integrating

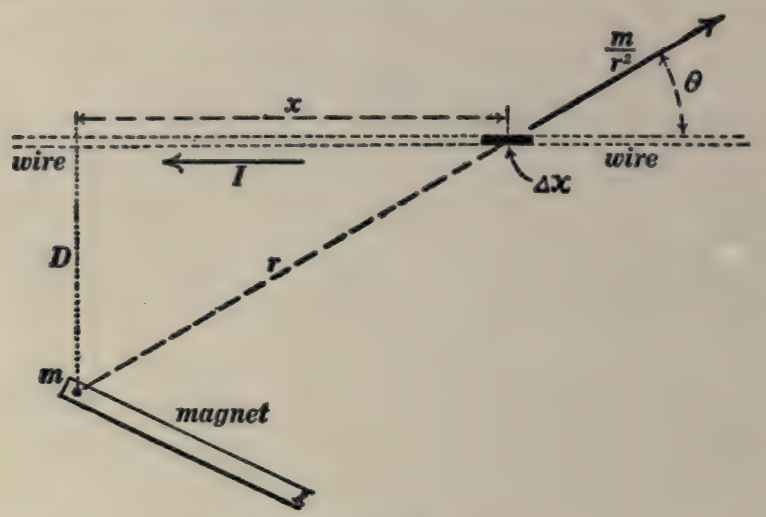

Fig. 23.

The field at $m$ due to $\Delta x$ is directed towards the reader.

equation (I) of Art. 18 as follows: Substitute $\Delta x$ for $\Delta l$, substitute $\left(D^{2}+x^{2}\right)$ for $r^{2}$, and substitute $\frac{D}{\sqrt{D^{2}+x^{2}}}$ for $\sin \theta$. Equation (I) of Art. 18 then becomes:

$$
\Delta H=I D \frac{\Delta x}{\left(D^{2}+x^{2}\right)^{3 / 2}}
$$

Therefore, by integrating between the limits $x=-\infty$ to $x=+\infty$ we have:

$$
H=\frac{2 I}{D}
$$

Thus at a distance of Io centimeters $(=D)$ from a long straight wire carrying a current of 50 abamperes $(=I)$ the intensity of the magnetic field is Io gausses $\left(=^{\circ} H\right)$.

20. Torque exerted on a coil which is suspended in a uniform magnetic field. Case I. Rectangular coil with two of its edges parallel to the field as shown in Fig. 24.-The forces FF in Fig. 24 are each equal to $l Z \times I \times H$ according to equation (I) of Art. I3, where $l$ is the dimension shown in Fig. 24, $Z$ is the number of 
turns of wire in the coil, $I$ is the current in the coil in abamperes, and $H$ is the intensity of the field in gausses. Multiplying

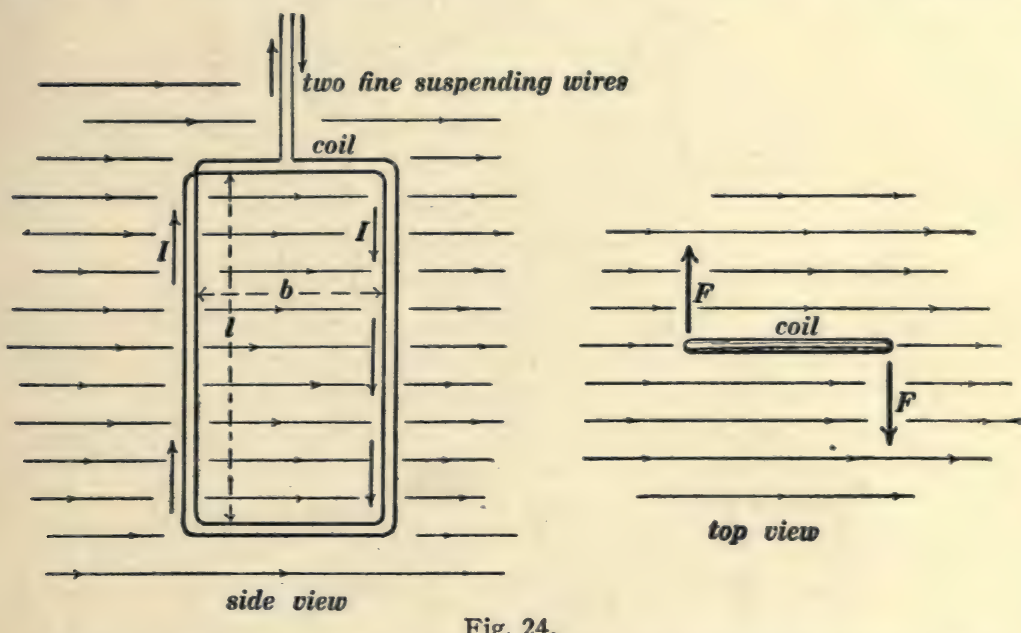

The two sides of the coil in the side view are pushed towards and away from the reader respectively as indicated in the top view.

each force $F$ by its lever arm $\frac{b}{2}$ and adding the two results, we get the torque $T$ which tends to turn the coil around the axis of suspension. That is:

$$
T=b l Z I H
$$

Case II. Circular coil with its plane parallel to the field as shown in Fig. 25. - The vertical dotted line in Figs. 25 and 26 is the axis about which the torque is to be determined. Consider an element $\Delta l$ of the coil. The length of wire in this element is $Z \cdot \Delta l$ where $Z$ is the number of turns of wire in the coil. The resolved part of $H$ at right angles to $\Delta l$ is $H \sin \theta$, and the side force $F$ (perpendicular to the plane of the paper in Fig. 26) exerted on $\Delta l$ is $Z \cdot \Delta l \times I \times H \sin \theta$. The perpendicular distance of $\Delta l$ from the axis in Fig. 26 is $r \sin \theta$, so that the torque action of $F$ is $\Delta T=F r \sin \theta$. That is:

$$
\Delta T=r Z I H \sin ^{2} \theta \cdot \Delta l
$$




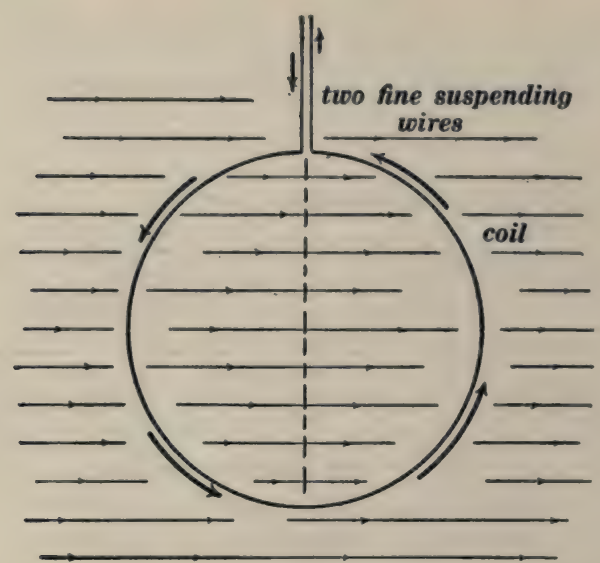

Fig. 25.

Circular coil of wire suspended in a uniform magnetic field.

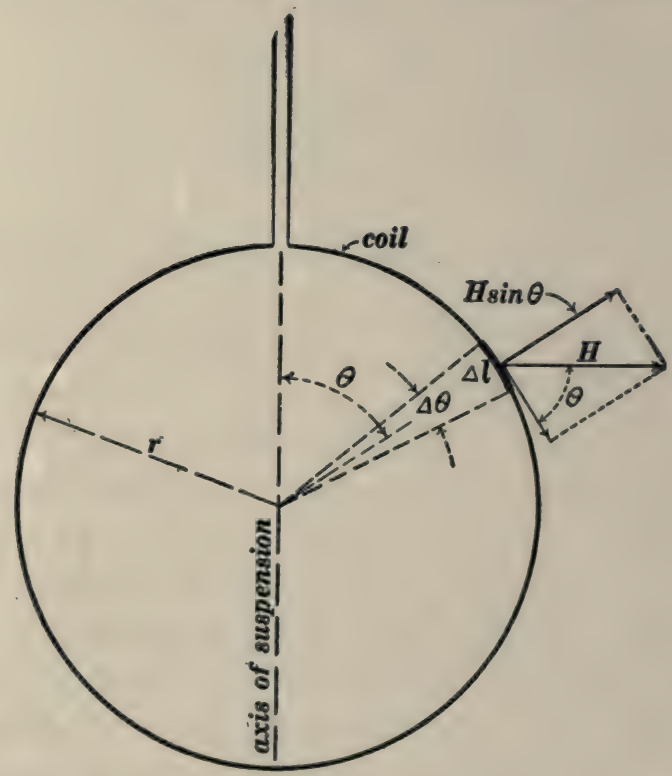

Fig. 26.

Let $\Delta \theta$ be the angle in radians which is subtended by $\Delta l$; then $\Delta l=r \cdot \Delta \theta$, and equation (2) becomes:

$$
\Delta T=Z I H r^{2} \sin ^{2} \theta \cdot \Delta \theta
$$


THE MAGNETIC MEASUREMENT OF CURRENT.

Therefore

$$
T=Z I H r^{2} \int_{\theta=0}^{\theta=2 \pi} \sin ^{2} \theta \cdot d \theta
$$

or

$$
T=\pi r^{2} Z I H
$$

Note.-When the field $H$ is at right angles to the axis about which the torque $T$ is reckoned but inclined to the plane of

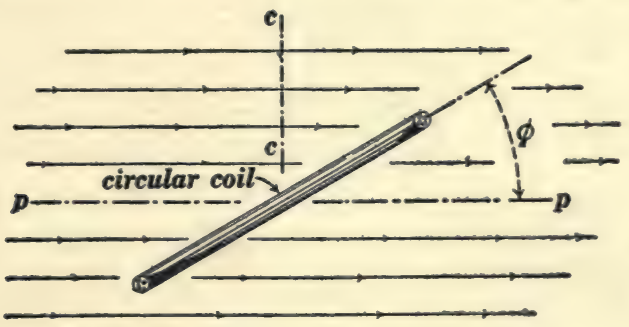

Fig. 27.

Top view of suspended coil.

the coil as shown in Fig. 27, then $H \cos \phi$ is the component of the field which lies in the plane of the coil, and substituting $H \cos \phi$ for $H$ in equations (I) or (5) we get the correct expression for $T$ for the case shown in Fig. 27.

2I. The Siemens electrodynamometer.-A general view of a Siemens electrodynamometer is shown in Fig. 28, and the essential features of the instrument are shown in Fig. 29. A stationary coil $A$ and a suspended coil $B$ are

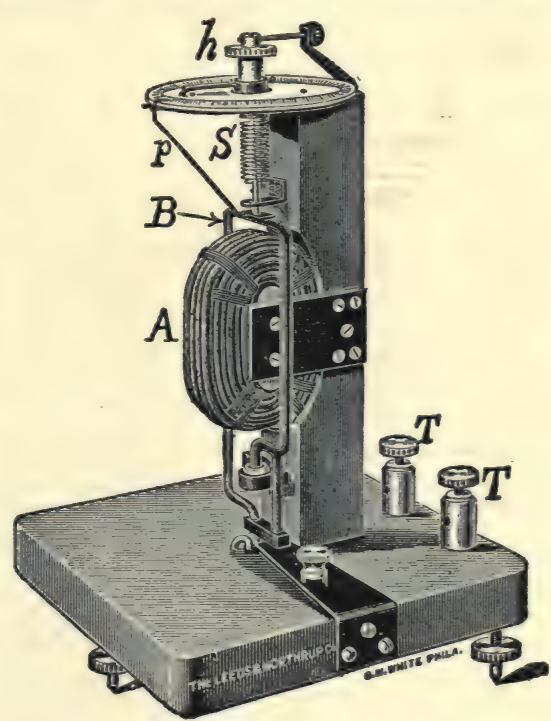

Fig. 28. 
connected electrically in series, and the current $I$ to be measured flows through both coils. The suspended coil $B$ is shown in both figures as a single turn of copper rod, and electrical con-

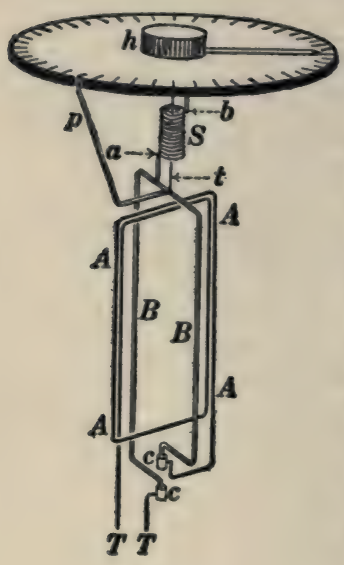

Fig. 29. nections are made with $B$ through the mercury cups $c c$. The coil $B$ is suspended by a fine thread $t$. A coiled spring $S$ is attached at one end to the suspended coil $B$ (at the point $a$ ), and the other end of $S$ is attached (at the point $b$ ) to the head $h$ so that by turning $h$ the spring $S$ can be twisted and the angle of twist $\phi$ can be read off the divided circle. When the spring $S$ is untwisted and when no current is flowing through $A$ and $B$, the pointer $p$ stands in a certain zero position. When the current $I$, to be measured, flows through $A$ and $B$ a torque or turning force is exerted on $B$ by $A$, and this torque is balanced by turning $h$ through an observed angle $\phi$ until the pointer $p$ again stands at its zero position. Then $I$ is proportional to $\sqrt{\phi}$, that is:

$$
I=k \sqrt{\phi}
$$

in which $k$ is a constant which is called the reduction factor of the instrument.

The correctness of equation (I) may be made evident as follows: The stationary coil $A$ produces a magnetic field which pushes sidewise on the vertical legs of coil $B$. If the current $I$ is doubled this field is doubled, and the doubled field acting on doubled current in coil $B$ pushes sidewise on the legs of $B$ with quadrupled force, according to equation (I) of Art. 13; but to balance this quadrupled force a quadrupled twist of the spring $S$ is necessary. Therefore $\phi$ is proportional to $I^{2}$, or $I$ is proportional to $\sqrt{\phi}$. 
22. The Weber electrodynamometer.-In order to measure a current by means of the Siemens electrodynamometer, the reduction factor of the instrument must be determined by observing the value of $\phi$ for a known value of the current $I$ [see equation (I) of Art. 2I]. The Weber electrodynamometer, on the other hand, is so constructed that the value of $k$ can be calculated from the dimensions of the coils $A$ and $B$ and their positions relative to each other. The Weber electrodynamometer is sometimes called an "absolute" electrodynamometer because the measurement of current by the Weber instrument involves only geometrical and mechanical measurements.

Imagine a large circular coil of radius $R$, and having $Z^{\prime}$ turns of wire, to be placed with its axis along $p p$ and its plane along $c c$ in Fig. 27, and let us think of the magnetic field $H$ of Fig. 27 as due to a current of $I$ abamperes in this large circular coil. Then

$$
H=\frac{2 \pi Z^{\prime} I}{R}
$$

according to equation (I) of Art. I4.

Let the coil which is shown in Fig. 27 be a comparatively small circular coil of radius $r$ and having $Z^{\prime \prime}$ turns of wire, and suppose this small coil and the large coil to be connected in series so that the current $I$ flows through both. Then the torque exerted on the small circular coil placed as shown in Fig. 27 will be:

$$
T=\pi r^{2} Z^{\prime \prime} I H \cos \phi
$$

according to equation (5) of Art. 20 as modified by the note at the end of that article. Therefore, substituting the value of $H$ from equation (I) in equation (2) we have:

$$
T=\frac{2 \pi^{2} r^{2} Z^{\prime} Z^{\prime \prime} I^{2} \cos \phi}{R}
$$

from which the value of $I$ in abamperes can be calculated when $T$ (a mechanical quantity), $r, R$ and $\phi$ have been measured, $Z^{\prime}$, and $Z^{\prime \prime}$ being known. 
Note.-Lord Rayleigh in his determination of the electrochemical equivalent of silver* used an electrodynamometer of which the essential features are shown in Fig. 30. A horizontal cir-

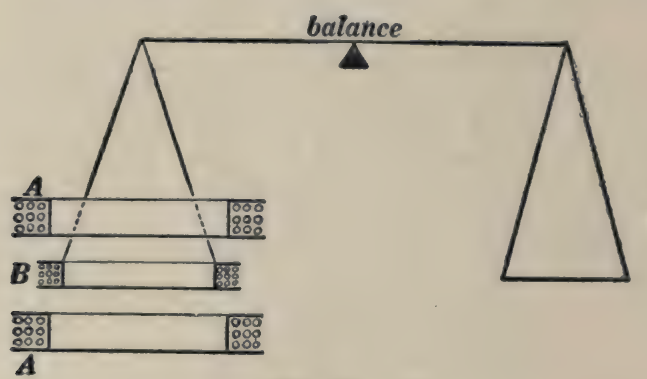

Fig. 30.

cular coil $B$ is suspended from one end of a balance arm and pushed up or down by the electromagnetic action of two stationary coils $A A$. All three coils are connected in series, and the value of the current is calculated from the observed push on $B$ and the sizes and relative positions of the coils.

\section{PROBLEMS.}

I. A circular coil of wire of 20 centimeters radius has 15 turns of wire. How much current is required in the coil to produce at the center of the coil a field intensity of 0.57 gauss? Ans. 0.I2I abampere.

2. A certain current gives a deflection of $42^{\circ}$ on a tangent galvanometer at a place where the horizontal component of the earth's magnetic field has a value of 0.18 gauss. The diameter of the coil of the galvanometer is 30 centimeters and the coil contains 5 turns of wire. Find the value of the current in abamperes. Ans. 0.0774 ampere.

Note-Assume the section of the coil itself to be very small; that is to say, take 30 centimeters as the mean diameter of the coil.

3. A tangent galvanometer has two coils of wire $A$ and $B$ placed side by side as shown in Fig. p3. The needle $n s$ of the

* See a paper on The Electrochemical Equivalent of silver by Lord Rayleigh and Mrs. H. Sidgwick, Philosophical Transactions, 1884, part II, pages 41 I-460. 
galvanometer is suspended midway between the coils as shown. The horizontal intensity $H^{\prime}$ of the earth's magnetic field is equal to o.I 8 gauss. The coils $A$ and $B$ each consist of one turn of wire. Find the deflection of the needle which is produced by a current of 0.12 abampere flowing in each coil. Ans. $16^{\circ} 40^{\prime}$.

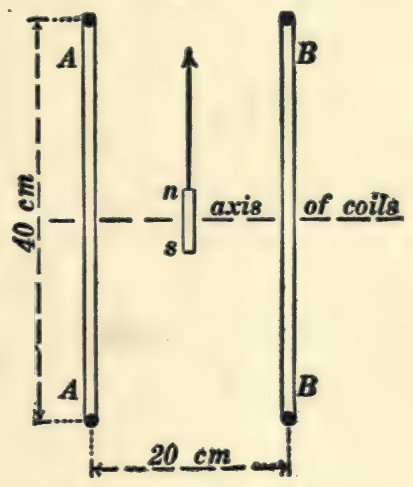

Fig. $p 3$.

4. A thin brass tube 2 inches in diameter and 6 feet long is wound with 1,400 turns of wire. Calculate the field intensity inside of this coil when a current of $\mathbf{5}$ amperes flows through the wire. Ans. 48.I gausses.

5. A long straight wire carrying a current of ro amperes is placed horizontally and magnetic north and south at a distance of 40 centimeters directly above a short compass needle. Find the deflection of the compass needle, the horizontal intensity of the earth's field at the compass needle being 0.18 gauss. Ans. $29^{\circ} 3^{\prime}$.

6. The two straight parallel wires of an electric light pole-line are 20 inches apart center to center, and a current of 500 amperes is flowing out in one wire and back in the other. Find: (a) The intensity of the magnetic field due to the wires at a point midway between them, and (b) the intensity of the magnetic field due to the two wires at a point which is 20 inches from the axis of one wire and 40 inches from the axis of the other wire. Ans. (a) 7.88 gausses. (b) 1.97 gausses. 
7. A rectangular frame $25 \times 40$ centimeters has Io turns of wire wound upon it. The frame is balanced horizontally upon an axis pointing due magnetic east and west. A current of 28 amperes is sent through the wire. Required the distance from the axis at which a Io-gram (9,800-dyne) weight must be hung to balance the torque action due to the earth's magnetic field at a place where its intensity is 0.57 gauss and its dip is $63^{\circ}$. Ans. 0.74 centimeter.

8. A circular coil has 100 turns of wire. The diameter of the mean turn is 16 centimeters, and a current of 15 amperes flows through the coil. This coil is suspended with its plane lying vertical and magnetic north and south. (a) Calculate the torque in dyne-centimeters with which the horizontal component of the earth's field (0.2 gauss) acts upon the coil, and specify the direction of the axis about which this torque is exerted.

Calculate the torque in dyne-centimeters with which the vertical component of the earth's field (o.68 gauss) acts on the coil, and specify the direction of the axis about which this torque is exerted. Ans. (a) Axis, vertical; torque, 6,032 dyne-centimeters. (b) Axis, north and south; torque, 20,508 dynecentimeters.

9. A circular coil to centimeters in diameter, having 50 turns of wire, is hung by a phosphor-bronze wire at the center of a large circular coil $\mathbf{I} 20$ centimeters in diameter, having 500 turns of wire. The suspending wire is free from twist when the planes of the two coils are at right angles, and a torque of 250 dynecentimeters twists the wire through one radian of angle. How much current must pass through the two coils in series to cause the suspended coil to turn $30^{\circ}$ from its position of equilibrium? What happens if the current is reversed in one coil? What happens if the current is reversed in both coils? Ans. 0.27 ampere. 


\section{CHAPTER III.}

ELEMENTARY THEORY OF ELECTROMAGNETISM.

INDUCED ELECTROMOTIVE FORCE AND INDUCTANCE.

23. Lenz's principle.-The wires on a dynamo armature are said to "cut" the magnetic lines of force as they move sidewise across the magnetic field in the gap space between the field poles and the armature core, and this "cutting" of the lines of force induces an electromotive force in the armature wires.

(a) In the motor, an electric current (from a battery, say) is forced through wires which are allowed to move sidewise across a magnetic field in the direction of the side push of the field on the wires. This motion of the wires across the field induces in them an electromotive force which opposes the flow of current, and the work done by the battery in overcoming this opposition all reappears as the mechanical work done by the side push as the wires move sidewise.

(b) In the generator, wires are pushed (by an engine, say) across a magnetic field. This motion induces an electromotive force in the wires, which in turn produces a current in the wires and in the outside circuit to which the generator is connected. This current causes the magnetic field to push sidewise on the moving wires in a direction opposite to their motion, and the work done by the engine in overcoming this opposition reappears as the electrical work done by the electromotive force in maintaining the induced current.

The two statements $(a)$ and $(b)$ express a principle which was first definitely pointed out by Lenz in 1834 , and it is called Lenz's principle.

24. Law of induced electromotive force.-Let $b c$, Fig. 3I, be a wire stretched across a magnetic field as shown, the intensity of the field being $H$ gausses. The side force $F$ (in dynes) 
exerted on the wire by the field is equal to $l I H$ dynes where $l$ is the length of the wire in centimeters and $I$ is the strength of the current in the wire in abamperes.

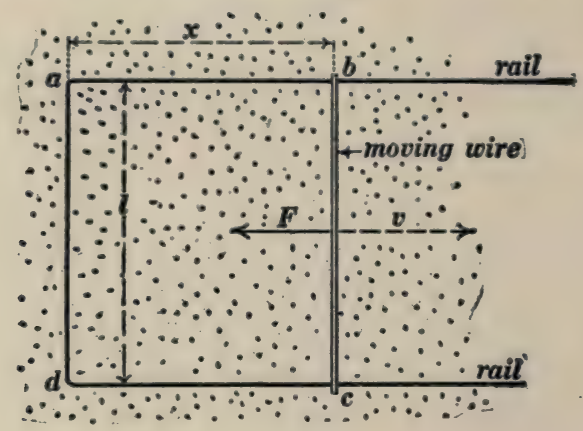

Fig. 31.

Dots represent magnetic lines of force which are perpendicular to the plane of the paper.

Suppose the wire to be moving in the direction of the dotted arrow at a velocity of $v$ centimeters per second. Then work will be done at the rate of $F v$ ergs per second in moving the wire in opposition to the force $F$; but, according to Lenz's principle, all of the work thus spent reappears as the electrical work done by the induced electromotive force in maintaining the current $I$. Let $E$ be the electromotive force in abvolts which is induced in the moving wire, then $E I$ is the rate at which work is done by the induced electromotive force in maintaining the current. Therefore, according to Lenz's principle we must have

whence we get

$$
E I=F v=l I H v
$$

$$
E \text { (in abvolts) }=l H v
$$

that is, the electromotive force $E$ in abvolts which is induced in the moving wire in Fig. $3 \mathrm{I}$ is equal to the product of the length $l$ of the wire in centimeters, the intensity, $H$, of the magnetic field in gausses, and the velocity, $v$, of the wire in centimeters per second. 
25. Definition of magnetic flux.-Figure 32 represents a plane area of $a$ square centimeters at right angles to a uniform magnetic field of which the intensity is $H$ gausses. The product $a H$ is called the magnetic flux across the area.

The unit of magnetic flux is the flux across an area of one square centimeter $(a=\mathrm{I})$ when the area is at right angles to a magnetic field of which the intensity is one gauss ( $H=\mathrm{I}$ ), or it is the flux across $n$ square centimeters of area when the area is at right angles to a magnetic

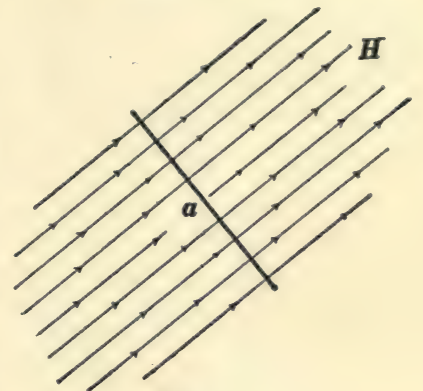

Fig. 32 . field of which the intensity is $I / n$th of a gauss. The unit of magnetic flux is properly called the maxwell; the common usage however is to call the unit of flux a line of force for the following reason:

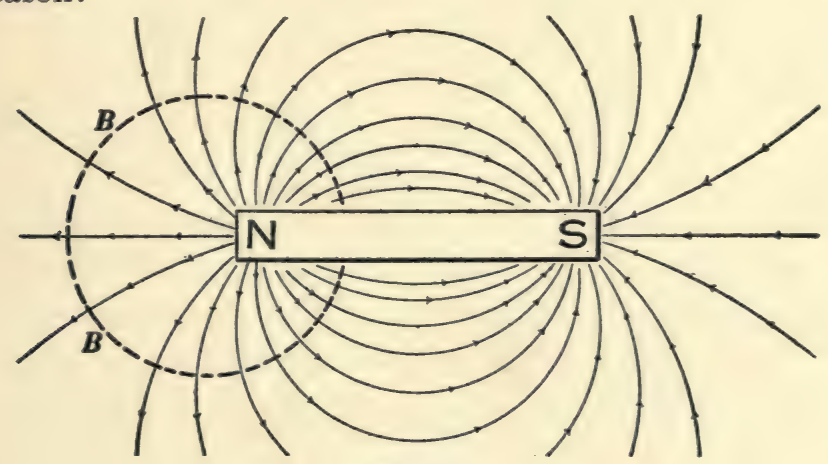

Fig. 33.

Consider any magnetic field whatever, and imagine a surface or shell $B B$ which is everywhere at right angles to the field as shown in Fig. 33. Imagine the surface $B B$ to be divided up into small squares such that one unit of magnetic flux crosses each square, and imagine lines of force to be drawn so that one line of force passes through each square. Then the magnetic flux across any other surface or shell $A A$ placed anywhere in the 
field will be equal to the number of these lines of force which cross AA.

26. Proposition.-The amount of magnetic flux $\Phi$ which emanates from a north magnet pole is equal to $4 \pi M$, where $M$ is the strength of the pole. Imagine a spherical surface of radius $r$ drawn with its center at the pole $M$ as shown in Fig. 34. The

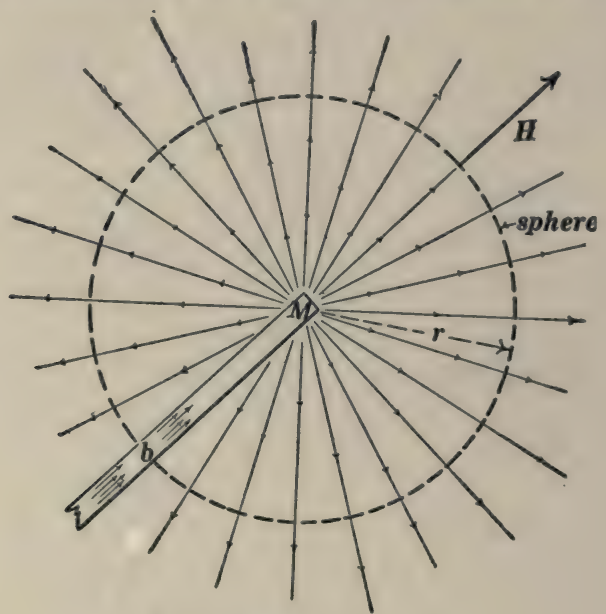

Fig. 34.

area of this spherical surface is $4 \pi r^{2}$ (neglecting the small portion of the sphere which falls inside of the slim magnet at $b$ ). The magnetic field at the spherical surface due to $M$ is everywhere at right angles to the surface, and its intensity at the surface is $H=\frac{M}{r^{2}}$ according to Art. 9. Therefore, the magnetic flux across the spherical surface is equal to $4 \pi r^{2} \times \frac{M}{r^{2}}$ or $4 \pi M$, according to Art. 25. But the flux across the spherical surface is the flux that emanates from the pole. Therefore the flux that emanates from the pole is $4 \pi M$.

Magnetic flux through the steel bar at $b$, Fig. 34.-The magnetic flux which emanates from the north pole $M$ in Fig. 34, enters the magnet at its south pole and passes along the bar to 
the nurth pole. Therefore the magnetic flux inside of the bar at $b$ is $4 \pi M$ lines.*

27. The electromotive force in abvolts which is induced in the moving wire $b c$ in Fig. $3 \mathrm{I}$ is equal to the rate at which the wire cuts magnetic flux.-Consider the sidewise distance $\Delta x$ moved by the wire $b c$ in Fig. 3I during the short time interval $\Delta t$. Then:

$$
\Delta x=v \cdot \Delta t
$$

The area swept over by the wire during the time interval $\Delta t$ is $l \cdot \Delta x$, and the amount of flux crossing this area is $\Delta \Phi=H l \cdot \Delta x$, or, using the value of $\Delta x$ from (I), we have:

$$
\Delta \Phi=H l v \cdot \Delta t
$$

and, of course, this is the amount of flux which is "cut" by the wire during the time interval $\Delta t$. Therefore, dividing $\Delta \Phi$ by $\Delta t$ we have the rate at which the wire cuts flux [in lines of force (or maxwells) per second], and from equation (2) we have:

$$
\frac{\Delta \Phi}{\Delta t}=H l v
$$

That is the rate at which the wire "cuts" flux is equal to $\mathrm{Hlv}$, but $H l v$ is the electromotive force in abvolts induced in the moving wire, according to equation (I) of Art. 24. Therefore the electromotive force in abvolts induced in the moving wire bc in Fig. 3I is equal to the number of lines of force (maxwells) cut by the wire per second.

28. Fundamental equation of the direct-current dynamo.The electromotive force induced in the armature winding of a direct-current dynamo is:

$$
E \text { (in abvolts) }=\Phi Z n
$$

in which $\Phi$ is the magnetic flux which enters the armature core from the north pole of the field magnet and leaves the armature core to enter the south pole of the field magnet as indicated by

* The bar magnet being very long and slim so that the small portion of the sphere which is inside of the bar at $b$ may be neglected. 
the lines of force in Fig. 35, $Z$ is the number of wires lying lengthwise on the outside surface of the armature, and $n$ is the speed of the armature in revolutions per second. This equation applies to the direct-current dynamo having a two-pole field magnet.* While one of the armature wires moves from $a$ to $b$ in Fig. 35

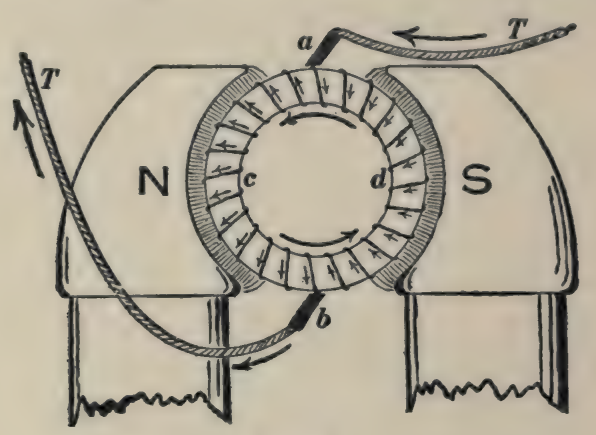

Fig. 35.

it cuts all of the magnetic flux $\Phi$ which enters the armature core from the north pole of the field magnet. The time of one revolution of the armature is $\mathrm{I} / n$th of a second, and the time required for a wire to move from $a$ to $b$ in Fig. 35 is $\mathrm{I} / 2 n$th of a second. Therefore, dividing $\Phi$ (the amount of flux cut) by $\mathbf{I} / 2 n$th of a second gives $2 n \Phi$ as the average rate of cutting of flux or the average electromotive force induced in an armature wire while it moves from $a$ to $b$. But the average electromotive force in $a$ given wire while it is moving from $a$ to $b$, is the same as the average electromotive force in all of the armature wires between $a$ and $b$ at any instant. Therefore $2 n \Phi$ is the average induced electromotive force in the wires $\left(\frac{1}{2} Z\right.$ in number) on the halfarmature $a c b$; but the wires on the half-armature are all in series, and therefore the electromotive force available at the brushes is $\frac{Z}{2} \times 2 n \Phi$ from which equation (I) follows at once.

The induced electromotive force $E$ in equation (I) may be

* The general form of the equation is discussed on pages 99-100 of Franklin and Esty's Dynamos and Motors, The Macmillan Co., New York, 1909. 
determined by connecting a voltmeter between the brushes of the dynamo and taking the reading of the voltmeter, provided the dynamo brushes are properly located and provided there is but very little current flowing through the armature. When a considerable current is flowing through the armature a portion of the total induced electromotive force is lost in overcoming the resistance of the armature windings.

29. Expression of induced elect omotive force in terms of the rate of change of the magne'ic flux which passes through a circuit.-Figure $3 \mathrm{I}$ shows a wire $b c$ sliding sidewise at a velocity of $v$ centimeters per second along two rails. The electromotive force induced in the moving wire is Hlv according to Art. 24, and this electromotive force acts to produce current in the circuit $a b c d$; the intensity of the magnetic field in Fig. 3I being $H$ gausses at right angles to the plane of the paper.

The area of $a b c d$ is $l x$ square centimeters, and the magnetic flux $\Phi$ which passes through the opening $a b c d$ is found by multiplying $H$ by the area $l x$; that is:

$$
\Phi=H l x
$$

Now if $x$ changes, $\Phi$ must change $H l$ times as fast, that is:

$$
\frac{d \Phi}{d t}=H l \frac{d x}{d t}
$$

But, $\frac{d x}{d t}$ is the velocity $v$ at which the wire moves sidewise. Therefore equation (2) becomes:

$$
\frac{d \Phi}{d t}=H l v
$$

Therefore, remembering that $H l v$ is the electromotive force induced in $b c$, and remembering that $\Phi$ is the magnetic flux through the opening $a b c d$, we have the following proposition: When the magnetic flux $\Phi$ through the opening of a circuit changes, an electromotive force is induced in the circuit, and 
this induced electromotive force in abvolts is equal to the rate of change of $\Phi$ in lines of force (maxwells) per second. That is:

$$
E=-\frac{d \Phi}{d t}
$$

Experiment shows that this equation is true not only when the change of magnetic flux is due to motion as in Fig. 31, but also when the change of magnetic flux is due to a varying magnetic field or to a varying state of magnetism of an iron core as in the transformer or induction coil.

The negative sign is chosen in equation (4) for the following reason: The flux $\Phi$ is thought of as going through the opening of a circuit in the direction of the magnetic field. Suppose that $\Phi$ is increasing. Then $\frac{d \Phi}{d t}$ is positive, and the induced electromotive force $E$ is in the direction around the circuit in which a lefthanded screw would have to be turned in order that it might travel in the direction of $\Phi$. If we choose the direction-through-theopening-of-a-circuit which is to be considered as positive, then the positive direction around the circuit is considered to be the direction in which a right-handed screw would have to be turned in order that the screw might travel in the positive direction through the opening of the circuit. Therefore when $\frac{d \Phi}{d t}$ is positive $E$ is negative.

Equation (4) expresses the electromotive force which is induced in a single turn of wire. When a region of changing magnetic flux is encircled by $Z$ turns of wire, then the induced electromotive force is multiplied $Z$ times, and equation (4) becomes:

$$
E=-Z \frac{d \Phi}{d t}
$$

in which $\frac{d \Phi}{d t}$ represents the rate of change of the flux in lines of force (maxwells) per second, and $E$ is the induced electromotive force in abvolts. 
30. Electromotive force relations of the alternating-current transformer.-Figure 36 is a diagram showing one coil of a transformer connected to I,IOO-volt alternating-current street mains, and the other coil of the transformer delivering current to house wires. A remarkable thing about a transformer is that the primary coil may have, say, one ohm of resistance and yet but very little current will flow through it when it is connected directly across I, Ioo-volt mains. As a matter of fact the electromotive force (the back electromotive force $b$ as described below) which is induced in the primary coil by the magnetic reversals of the transformer core is at each instant opposite to and very nearly equal to the electromotive force across the supply mains.

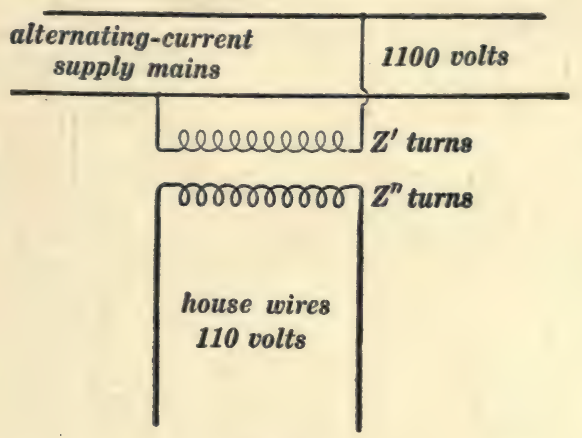

Fig. 36.

It is very difficult to make an intelligible argument concerning an alternating electromotive force or current unless some simple scheme is used to represent successive instantaneous values of electromotive force or current. Imagine the line $E^{\prime}$, Fig. 37 , to rotate uniformly about $O$ in the direction of the arrow $f$, the number of revolutions per second of the line being equal to the number of cycles per second of the alternating electromotive force across the supply mains in Fig. 36 . Then the length of $E^{\prime}$ may be chosen so that its projection $e^{\prime}$ on the fixed line $c d$ represents the actual electromotive force between the supply mains in Fig. 36 at each instant. 
The back electromotive force $b$ which is induced in the primary coil of the transformer is very nearly equal and opposite to $e^{\prime}$ at each instant. For the sake of simplicity let us think of the back electromotive force $b$ as being exactly equal and opposite to $e^{\prime}$ at each instant. Then $b$ will be represented at each instant by the projection of the rotating line $O B$ which is equal in length to $E^{\prime}$ and always opposite in direction to $E^{\prime}$.

In the above discussion $b$ is the value at any instant of the electromotive force induced in the primary coil. Let $e^{\prime \prime}$ be the value at any instant of the electromotive force induced in the secondary coil. Then $e^{\prime \prime}$ is smaller than $b$ in the same ratio that $Z^{\prime \prime}$. is smaller than $Z^{\prime}$ (see Fig. 36), because a definite

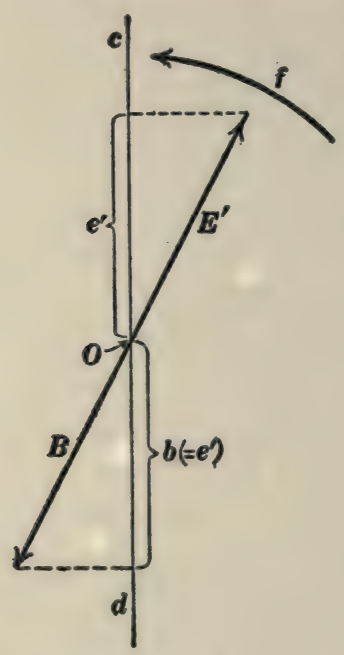

Fig. 37 .

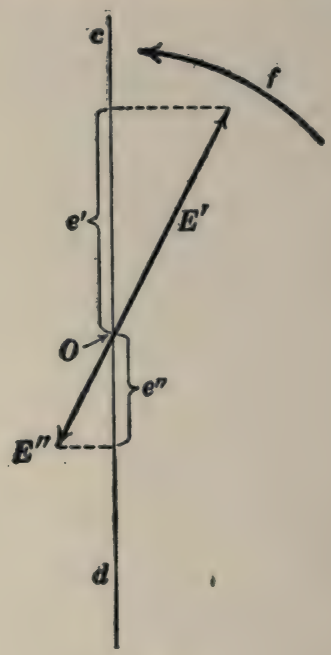

Fig. 38.

amount of electromotive force is induced in each turn of wire that encircles the core. Therefore the electromotive force $e^{\prime \prime}$ may be represented at each instant by the projection of the rotating line $E^{\prime \prime}$ which is parallel to $O B$ and $\frac{Z^{\prime \prime}}{Z^{\prime}}$ as long as $O B$. That is, the line $E^{\prime \prime}$ whose projection represents $e^{\prime \prime}$ at each instant is always opposite to the rotating line $E^{\prime}$ whose projection repre- 
sents $e^{\prime}$, and $E^{\prime \prime}$ is $\frac{Z^{\prime \prime}}{Z^{\prime}}$ as long as $E^{\prime}$, as shown in Fig. 38 .

Figure 38 applies to a transformer in which $Z^{\prime}=2 Z^{\prime \prime}$.

3r. Magnetic flux through the opening of a circuit due to the current in the circuit. Definition of inductance.-Consider a coil consisting of a single turn of wire as shown in Fig. 39. The
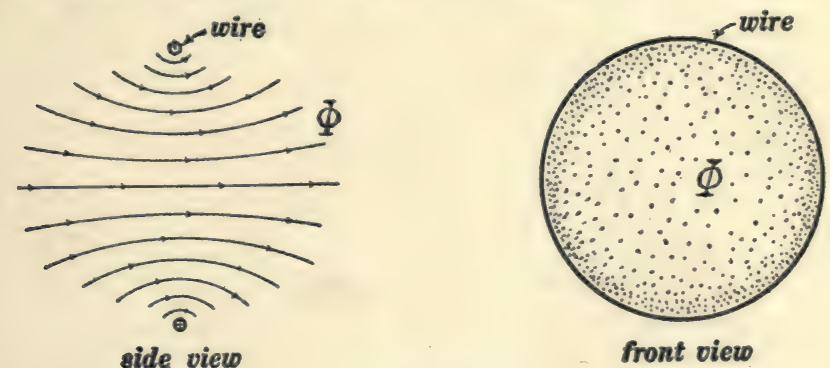

Fig. 39.

lines of force of the magnetic field due to a current in the coil are shown in the side view. The amount of magnetic flux $\Phi$ through the opening of the coil is proportional to the current $I$ in the coil. That is, we may write:

$$
\Phi=L I
$$

where $L$ is a proportionality factor which depends on the size and shape of the circuit. It is called the inductance of the circuit.

Equation (I) is exactly true only when there is no iron in the neighborhood of the coil. Therefore, the remainder of this chapter applies strictly to electric coils or circuits without iron.

It is best to use c.g.s. units (abamperes, abvolts, etc.) in any equation involving magnetic field intensity $H$ or magnetic flux $\Phi$, because the units of $H$ and $\Phi$ which are generally used, namely, the gauss and the maxwell (or line of force), are c.g.s. units. The c.g.s. unit of inductance is called the abhenry, and $L$ in equation ( $\mathrm{I}$ ) is expressed in abhenrys when $\Phi$ is expressed in maxwells (lines of force) and $I$ in abamperes.

When the coil has more than one turn of wire the expression 
"flux through the opening of the coil" has a somewhat complicated meaning. It is a fundamental principle in geometry that in considering the area of a multiple loop each part of the area must be counted $n$ times, where $n$ is the number of loops encircling that part. Thus the shaded area $A$ in Fig. 40 would

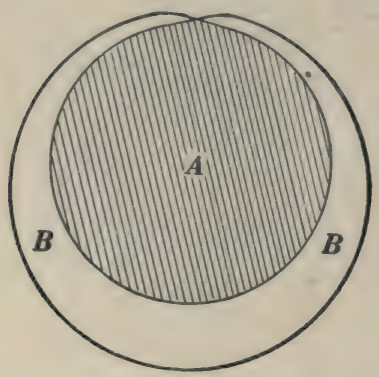

Fig. 40 .

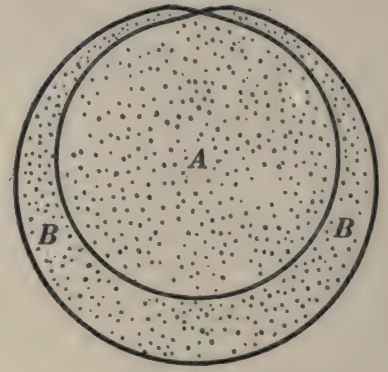

Fig. 41.

be counted twice, and the area $B$ would be counted once. Similarly in considering the magnetic flux through the opening of a coil each part of the flux is counted $n$ times where $n$ is the number of turns of wire encircling that part. Thus the flux $A$ in Fig. $4 \mathrm{I}$ would be counted twice and the flux $B$ would be counted once. If the flux $A$ is one million lines (maxwells) and if $B$ is half a million lines, then the flux $\Phi$ through the opening of the coil would be thought of as $(2 A+B)$ or $2,500,000$ lines. With this explanation of the meaning of $\Phi$, equation (I) becomes entirely general, and the definition of inductance as above given becomes entirely general.

Another point of view. Mean flux per turn.-In many cases it is convenient to consider the average or mean flux per turn of wire in a coil, and to multiply this by the number of turns to get the quantity $\Phi$ as above defined. Let $\Phi^{\prime}$ be the average or mean flux per turn, then the quantity $\Phi$, as above defined, is equal to $Z \Phi^{\prime}$ where $Z$ is the number of turns of wire in the coil. Therefore equation (I) may be written:

$$
Z \Phi^{\prime}=L I
$$


Example.-A coil is made by winding a layer of insulated wire on a very long wooden or paper cylinder. The length of the coil is $l$ centimeters, its radius is $r$ centimeters and the number of turns of wire is $Z$. What is its inductance in abhenrys? The number of turns of wire per centimeter is $\frac{Z}{l}[=z$ of equation (2) of Art. 17]. Therefore, according to Art. I7, the intensity of the magnetic field inside of the long coil is $H=4 \pi \times \frac{Z}{l} \times I$. Therefore the amount of magnetic flux passing through the middle part of the coil is $\pi r^{2} H$ or $4 \pi^{2} r^{2} \times \frac{Z}{l} \times I$. But this flux is encircled by $Z$ turns of wire. Therefore $Z \Phi^{\prime}$ is equal to $\frac{4 \pi^{2} r^{2} Z^{2}}{l} \times I$, and this is equal to $L I$ according to equation (2) above. Therefore we have:

$$
L=\frac{4 \pi^{2} r^{2} Z^{2}}{l}
$$

which expresses the value of $L$ in abhenrys. This equation is only approximate because the flux $\pi r^{2} H$ which passes through the middle part of the coil spreads out and does not all pass through the turns near the ends of the coil as shown in Fig. 42.

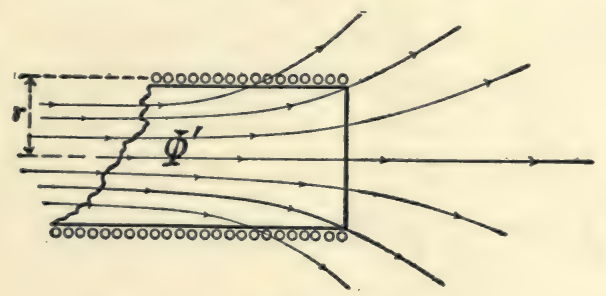

Fig. 42.

32. Self-induced electromotive force.-When the current in a circuit increases, the flux through the opening of the circuit also increases, according to equation (I) of Art. 3I. Let us base our discussion, however, on equation (2) of Art. 3I, where $Z$ 
is the number of turns of wire in a coil and $\Phi^{\prime}$ is the average flux per turn. Differentiating* this equation, we have:

$$
Z \frac{d \Phi^{\prime}}{d t}=L \frac{d I}{d t}
$$

Now $\Phi^{\prime}$ has here exactly the same meaning that $\Phi$ has in equation (5) of Art. 29. Therefore $-\frac{d \Phi^{\prime}}{d t}$ is the electromotive force which is induced in each turn of wire, and $-Z \frac{d \Phi^{\prime}}{d t}$ is the electromotive force $e$ which is induced in the entire coil by the changing current. Therefore from equation (I) we have:

$$
e=-L \frac{d I}{d t}
$$

When the current in a circuit is increasing, then $\frac{d I}{d t}$ is positive and the negative sign indicates that $e$ is opposed to the current. That is, an increasing current induces in a circuit an opposing electromotive force which is equal to $L$ times the rate of increase of the current. A decreasing current induces in a circuit a helping electromotive force which is equal to $L$ times the rate of decrease of the current.

33. Electromotive force required to make a current increase. Imagine a boat to move without frictional opposition; then the propelling force would be used wholly to cause the velocity to increase (to produce acceleration), and we would have:

$$
E=L \frac{d I}{d t}
$$

Imagine a circuit having no resistance; then an electromotive force acting on the circuit would be used wholly to cause the current to increase, and we would have:

$$
E=L \frac{d I}{d t}
$$

* This simple case of differentiation occurs so frequently in physical arguments that it is very important that the student understand it as a proposition in arithmetic. If $\Phi^{\prime}$ is always $\frac{L}{Z}$ times as large as $I$, then it must change $\frac{L}{Z}$ times as fast as $I$. That is we must have $\frac{d \Phi^{\prime}}{d t}=\frac{L}{Z} \cdot \frac{d I}{d t}$ or $Z \frac{d \Phi^{\prime}}{d t}=L \frac{d I}{d t}$. 
where $E$ is the propelling force, $L$ is the mass of the boat, and $\frac{d I}{d t}$ is the rate of increase of the velocity. If $\frac{d I}{d t}$ is negative it signifies decreasing velocity. where $E$ is the electromotive force acting on the circuit (of zero resistance), $L$ is the inductance of the circuit, and $\frac{d I}{d t}$ is the rate of increase of the current. If $\frac{d I}{d t}$ is negative it signifies decreasing current.

The units of force, mass and acceleration which are used in equation (I) are familiar to the student of elementary mechanics.

When the rate of increase of current in equation (2) is expressed in abamperes per second and $E$ in abvolts, then $L$ is expressed in abhenrys. That is to say, a circuit has an inductance of one abhenry when an electromotive force of one abvolt (in excess of the electromotive force $R I$ required to overcome the resistance of the circuit) will cause the current in the circuit to increase at the rate of one abampere per second.

When the rate of increase of current is expressed in amperes per second and $E$ in volts, then $L$ is expressed in henrys. - That is to say, a circuit has an inductance of one henry when an electromotive force of one volt (in excess of the electromotive force $R I$ required to overcome the resistance of the circuit) will cause the current in the circuit to increase at the rate of one ampere per second. One henry is equal to $10^{9}$ abhenrys.

\section{Further discussion of self-induced electromotive force.-} According to Art. 32 an electromotive force equal to $-L \frac{d I}{d t}$ is induced in a circuit by a changing current, and according to Art. 33 an electromotive force equal to $+L \frac{d I}{d t}$ must act on a circuit (in addition to the electromotive force $R I$ required to overcome resistance) to cause the current to increase. This matter may be made clear as follows: 
When a boat moves at velocity $I$, the drag of the water opposes the motion of the boat, and this frictional opposition is approximately proportional to I.* Therefore the propelling force $E$ must be equal to $R I$ to maintain the motion of the boat, $R$ being a constant.

When the velocity of a boat is increasing at the rate $\frac{d I}{d t}$, then the propelling force must exceed $R I$ (the backward drag of the water) by an amount which is equal to $L \frac{d I}{d t}$, where $L$ is the mass of the boat and $\frac{d I}{d t}$ is the rate at which the velocity of the boat is increasing.

The backward drag of the water on a canal boat shows itself as a backward pull by the boat on the mule which draws the boat. This backward pull of a boat due to friction may be called the resistance reaction of the boat and it is equal to $R I$.

When the speed of a canal boat is increasing at the rate
When a current $I$ flows through a circuit, something analogous to a frictional resistance opposes the flow of current, and to maintain the current an electromotive force equal to $R I$ must act on the circuit according to Ohm's law.

When a current in a circuit is increasing at the rate $\frac{d I}{d t}$, then the electromotive force which acts on the circuit must exceed $R I$ by an amount which is equal to $L \frac{d I}{d t}$, where $L$ is the inductance of the circuit and $\frac{d I}{d t}$ is the rate at which the current is increasing.

When a current is pushed through a circuit, the resistance of the circuit shows itself as a resistance reaction which is equal to $R I$.

When the value of the current in a circuit is increasing at

* The backward drag of the water on the moving boat is by no means proportional to the velocity of the boat except when the velocity is quite small. The exact proportional relationship is here assumed for the sake of the analogy. 
$\frac{d I}{d t}$, the pull of the mule must exceed $R I$ by an amount $L \frac{d I}{d t}$ as stated above, and the boat pulls back on the mule with a force which exceeds $R I$ by an amount $L \frac{d I}{d t}$. This additional backward pull of the boat on the mule $\left(L \frac{d I}{d t}\right)$ is called the inertia reaction of the boat. the rate $\frac{d I}{d t}$, the propelling electromotive force must exceed $R I$ by an amount $L \frac{d I}{d t}$ as stated above, because the propelling electromotive force must overcome not only the resistance reaction $R I$ of the circuit, but it must also overcome the self-induced electromotive force due to the increasing current.

Self-induced electromotive force in a circuit is exactly analogous to the inertia reaction of a boat.

\section{Kinetic energy of a current in a circuit.}

Work done on a body which moves without friction would all go to increase the kinetic energy stored in the body.

If $W$ is the amount of kinetic energy stored in a body, then $\frac{d W}{d t}$ (the rate of increase of $W$ ) is the rate at which work is done on the body to cause its speed to increase.

The rate at which work is done by a propelling force $E$ is $E I$, where $I$ is the velocity of the body. Therefore, using the value of $E$ from equation (I) of Art. 33, and remembering that the rate at which work
Work done on a circuit which has zero resistance would all go to increase the kinetic energy stored in the circuit.

If $W$ is the amount of kinetic energy stored in a circuit, then $\frac{d W}{d t}$ (the rate of increase of $W$ ) is the rate at which work is done on the circuit to cause the current to increase.

The rate at which work is done by a "propelling" electromotive force $E$ is $E I$, where $I$ is the current flowing in the circuit. Therefore, using the value of $E$ from equation.(2) of Art. 33, and remembering 
is done on a body is equal to $\frac{d W}{d t}$, as above explained, we have:

$$
\frac{d W}{d t}=L I \frac{d I}{d t}
$$

The kinetic energy of a bcdy which is moving at velocity $I$ is:

$$
W=I / 2 L I^{2}
$$

where $-L$ is the mass of the body. This equation may be established as follows:

Integrating equation (I), we have:

- $W=\mathrm{I} / 2 L I^{2}+$ a constant (5) but we are here concerned with the energy which depends upon $I$, and which is zero when $I$ is zero. The constant term in equation (5) therefore disappears and equation (5) reduces to equation (3).

\section{The starting of a boat.}

At a certain instant a constant force $E$ begins to act on a boat, and it is required to find an algebraic expression for the increasing velocity $i$ of the boat, on the assumption that the frictional drag of the water is exactly proportional to $i$, or equal to $R i$. that the rate at which work is done on a circuit is equal to $\frac{d W}{d t}$, as above explained, we have:

$$
\frac{d W}{d t}=L I \frac{d I}{d t}
$$

The kinetic energy of a circuit in which a current $I$ is flowing is:

$$
W=\mathrm{I} / 2 L I^{2}
$$

where $L$ is the inductance of the circuit. This equation may be established as follows:

Integrating equation (2), we have:

$$
W=\mathrm{r} / 2 L I^{2}+\mathrm{a} \text { constant }
$$

but we are here concerned with the energy which depends on $I$, and which is zero when $I$ is zero. The constant term in equation (6) therefore disappears and equation (6) reduces to equation (4).

Growth of current in a circuit. At a certain instant a constant electromotive force $E$ begins to act on a circuit, and it is required to find an algebraic expression for the growing current $i$. 
If we subtract the force which is required to overcome the backward drag of the water (namely, Ri) from the total propelling force $E$ we get the portion of the propelling force which is used to cause the velocity of the boat to increase. Therefore, according to Art. 33, we have:

$$
E-R i=L \frac{d i}{d t}
$$

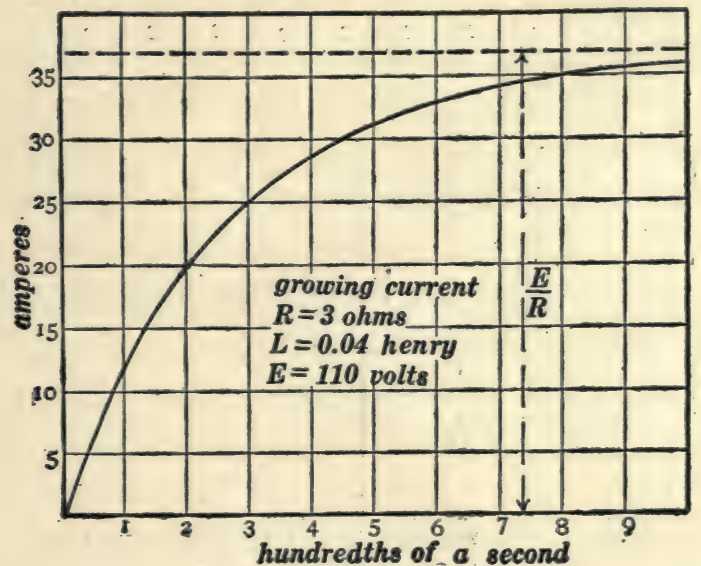

Fig. 43a.

The general solution of this differential equation, ${ }^{*}$ subject to the condition that the velocity of the boat is zero at the beginning ( $i=0$ when $t=0$ ), is:

* See Franklin, MacNutt and Charles's Calculus, pages I68-I8I (published by the authors, South Bethlehem, Pa., I913). The general solution of this
differential equation, ${ }^{*}$ subject
to the condition that the cur-
rent in the circuit is zero at the
beginning ( $i=0$ when $t=0$ ),
is:
If we subtract the voltage which is required to overcome the resistance of the circuit (namely, $R i$ ) from the total propelling electromotive force $E$ we get the portion of the propelling electromotive force which is used to cause the current to increase. Therefore, according to Art. 33, we have:

$$
E-R i=L \frac{d i}{d t}
$$




$$
i=\frac{E}{R}-\frac{E}{R} \cdot \epsilon^{-(R / L) \cdot t}
$$

where $R$ is the proportionality factor in equation (I), $L$ is the mass of the boat, $E$ is the constant propelling force, and $i$ is the velocity of the boat $t$ seconds after $E$ begins to act.

$$
i=\frac{E}{R}-\frac{E}{R} \cdot \epsilon^{-(R / L) \cdot t}
$$

where $R$ is the resistance of the circuit, $L$ is the inductance of the circuit, $E$ is the constant propelling electromotive force, and $i$ is the current in the circuit $t$ seconds after $E$ begins to act.

Equation (4) is plotted as a curve in Fig. 43a, in which abscissas represent elapsed times $t$ and ordinates represent the increasing values of the current $i$.

\section{The stopping of a boat.}

A boat is moving at given velocity $I$, and the propelling force suddenly ceases to act. It is required to find an algebraic expression for the decreasing velocity $i$ of the boat, on the assumption that the frictional drag of the water is exactly proportional to $i$, or equal to $R i$.

The frictional drag of the water is a force $R i$ which opposes $i$. Therefore the force $R i$ is to be considered as negative, and, according to Art. 33, we have:

$$
-R i=L \frac{d i}{d t}
$$

It is evident from this equation
Decay of current in a circuit.

A current $I$ is flowing in a circuit, and the propelling electromotive force suddenly ceases to act. It is required to find an algebraic expression for the decreasing current $i$ in the circuit.

The flow of current is opposed by a kind of frictional drag (an electromotive force) which is equal to Ri. Therefore the electromotive force $R i$ is to be considered as negative, and, according to Art. 33, we have:

$$
-R i=L \frac{d i}{d t}
$$

It is evident from this equation 
that $\frac{d i}{d t}$ must be negative, that $\mid$ that $\frac{d i}{d t}$ must be negative, that is $\frac{d i}{d t}$ is the rate of decrease of the velocity of the boat. is $\frac{d i}{d t}$ is the rate of decrease of the current in the circuit.

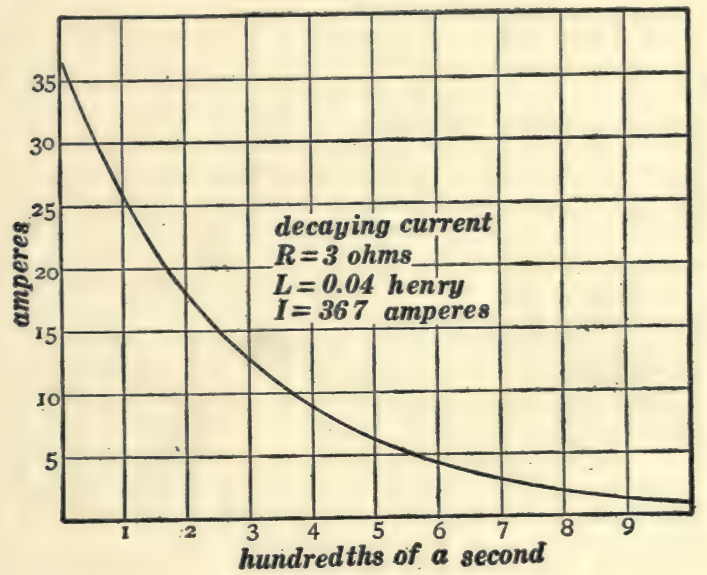

Fig. $43 b$.

The general solution of equation (I) which satisfies the necessary condition that the velocity of the boat is equal to $I$ at the beginning $(i=I$ when $t=0$ ), is:

$$
i=I \epsilon^{-(R \mid L) \cdot t}
$$

where $R$ is the proportionality factor in equation (I), $L$ is the mass of the boat, $I$ is the velocity of the boat when the propelling force ceases to act, and $i$ is the velocity of the boat $t$ seconds after the propelling force ceases to act.
The general solution of equation (2) which satisfies the necessary condition that the current in the circuit is equal to $I$ at the beginning $(i=I$ when $t=0$ ), is:

$$
i=I \epsilon^{-(R / L) \cdot t}
$$

where $R$ is the resistance of the circuit, $L$ is the inductance of the circuit, $I$ is the current in the circuit when the propelling electromotive force ceases to act, and $i$ is the value of the current $t$ seconds after the propelling electromotive force ceases to act. 
Equation (4) is plotted as a curve in Fig. $43 b$.

Note.-Something should be said as to how the propelling electromotive force may be made to cease as assumed in the above discussion of equations (2) and (4). It is not by breaking the circuit, because to do this would increase the resistance $R$ indefinitely. In order that the current in a circuit may die away according to equation (4) the battery must first be short-circuited by a metal connector; and after being short-circuited in this way the battery itself may be disconnected.

38. The ratio of the inductances of two entirely similar coils is equal to the ratio of their corresponding dimensions.-To illustrate the meaning of this proposition consider two exactly

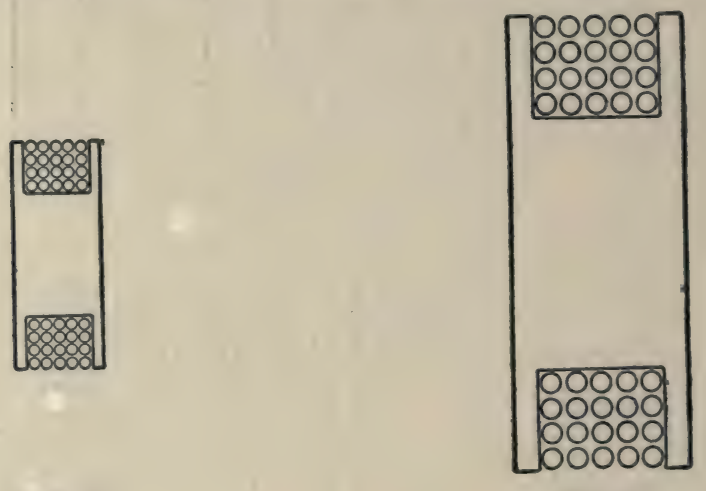

Fig. 44.

Fig. 45.

similar coils as shown in Figs. 44 and 45. The coil in Fig. 45 is twice as long and twice as deep, and wound with wire twice as large in diameter as the coil in Fig. 44, and the inductance of the coil in Fig. 45 is twice as great as the inductance of the coil in Fig. 44 .

Of course the two coils in Figs. 44 and 45 have the same number of turns of wire.

The above proposition may be proven on the basis of equation (I) in Art. 18, by showing that the field intensity at any point in Fig. 45 is half as great as at the corresponding point in Fig. 44 
for the same strength of current in the wire; from which it follows that the magnetic flux through each turn of wire in Fig. 45 is twice as great as the magnetic flux through the corresponding turn of wire in Fig. 44 for the same current in the wires. Therefore the value of $Z \Phi^{\prime} \quad$ (= LI according to Art. 3I) in Fig. 45 is twice as great as in Fig. 44 for the same strength of current in the wire. Therefore $L$ in Fig. 45 is twice as great as $L$ in Fig. 44.

The inductance of a coil of given size and shape is proportional to the square of the number of turns of wire.-To understand this proposition consider Figs. 46 and 47. These figures represent

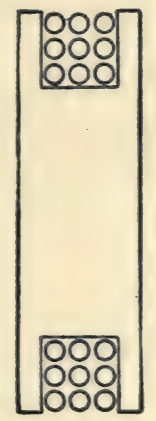

Fig. 46.

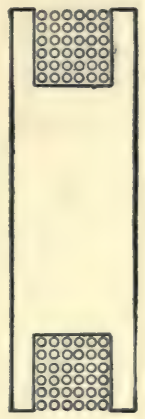

Fig. 47.

a spool of given size and shape wound full of coarse wire and of fine wire respectively. In fact, the diameter of the wire in Fig. 47 is half as great as the diameter of the wire in Fig. 46. Therefore there are twice as many turns in each layer of wire, and twice as many layers; and consequently four times as many turns of wire in Fig. 47. Therefore, according to the above proposition, the coil in Fig. 47 has sixteen times as much inductance as the coil in Fig. 46.

The above proposition may be established as follows: Let the coil in Fig. 47 contain $n$ times as many turns of wire as the coil in Fig. 46. Then each particular turn of wire in Fig. 46 corresponds to a bundle of $n$ wires in Fig. 47 , and to have a current 
of $I$ amperes in the coil of Fig. 47 is exactly the same as to have $n I$ amperes in the coil of Fig. 46. Now with a current of $n I$ in Fig. 46 the total kinetic energy is $\frac{1}{2} L^{\prime}(n I)^{2}$, and with a current of $I$ amperes in Fig. 47 the total kinetic energy is $\frac{1}{2} L^{\prime \prime} I^{2}$; and these two amounts of energy are equal according to the above statement. Therefore we have:

$$
\frac{1}{2} L^{\prime}(n I)^{2}=\frac{1}{2} L^{\prime \prime} I^{2}
$$

or

$$
n^{2} L^{\prime}=L^{\prime \prime}
$$

where $L^{\prime}$ is the inductance of the coil in Fig. 46 and $L^{\prime \prime}$ is the inductance of the coil in Fig. 47. Therefore according to equation (I) the inductance of the coil in Fig. 47 is $n^{2}$ times as great as the inductance of the coil in Fig. 46.

39. The inductance of a transmission line.-The inductance of a two-wire transmission line in henrys per mile is:

$$
L=0.001483 \log _{10}\left(\frac{D-R}{R}\right)
$$

where $D$ is the distance of the wires apart center to center, and $R$ is the radius of each wire; $D$ and $R$ must both be expressed in the same unit. This equation is only approximately true, as will appear in the following discussion.

Figure 48 is a sectional view of the two wires $W^{\prime}$ and $W^{\prime \prime}$ of a transmission line with an outflowing current of $I$ abamperes in one wire and an equal inflowing current in the other wire. In order to determine the inductance in abhenrys of one mile of the line it is sufficient to calculate the magnetic flux $\Phi$ which passes between* the wires for the given current $I$ : Then $\Phi=L I$ or $L=\frac{\Phi}{I}$ according to Art. $3 \mathrm{I}$.

Now the magnetic flux between the wires is the flux which crosses the plane which is represented by the dotted line $P P$

* The flux which crosses through the material of the wires is neglected in this discussion, and therefore equation (I) is only approximately true. 
in Fig. 48, and to calculate this flux let us consider an element of this plane $\Delta x$ centimeters wide and $16 \mathrm{I}, 000$ centimeters long (= one mile), the distance of the element from the axes of the

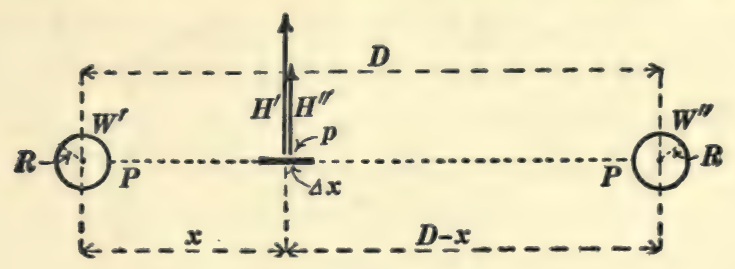

Fig. 48.

wires being $x$ and $D-x$ centimeters respectively. The magnetic field intensity at the element $\Delta x$ due to the wire $W^{\prime}$ is:

$$
H^{\prime}=\frac{2 I}{x}
$$

and the magnetic field intensity at the element $\Delta x$ due to the wire $W^{\prime \prime}$ is:

$$
H^{\prime \prime}=\frac{2 I}{D-x}
$$

according to Art. 19. The total magnetic field intensity at the element is $H^{\prime}+H^{\prime \prime}$, and the flux across the element is $\left(H^{\prime}+H^{\prime \prime}\right)$ multiplied by the area of the element, namely 161,000 $\Delta x$ square centimeters. Therefore:

$$
\Delta \Phi=322,000 I\left(\frac{\Delta x}{x}+\frac{\Delta x}{D-x}\right)
$$

Integrating this expression from $x=R$ to $x=D-R$, we get:

$$
\Phi=644,000 I \log _{e}\left(\frac{D-R}{R}\right)
$$

Therefore, since $\frac{\Phi}{I}=L$ according to Art. 3I, we have:

$$
L=644,000 \log _{\mathrm{e}} \frac{D-R}{R}
$$


This gives the inductance of one mile of the line in abhenrys. To reduce to henrys divide by $10^{9}$, and then reduce the Naperian logarithm to a common logarithm and we get equation (I).

40. Mechanical analog of the transformer.-The use of the lever for the multiplication of mechanical force is familiar to everyone, and the alternating-current transformer is mathematically analogous to the lever; not however to a lever with an immovable fulcrum, but to a lever of which the fulcrum is a very massive body entirely free to move as shown in Fig. 49

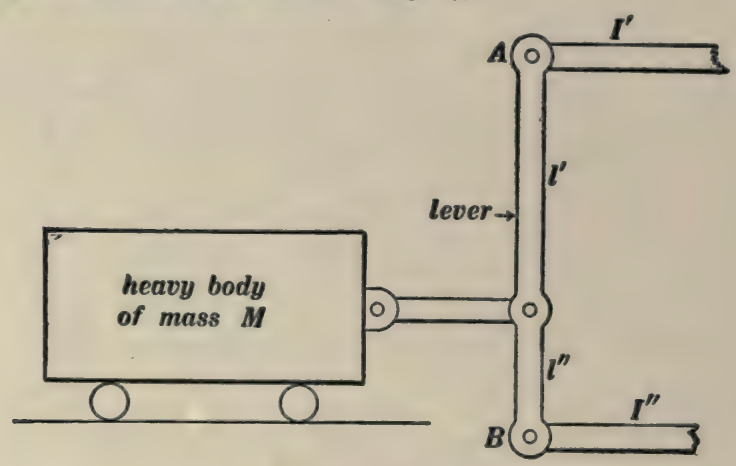

Fig. 49.

A steady force would eventually produce an indefinitely large velocity of the body $M$ (wheels supposed to be frictionless). Therefore the lever shown in Fig. 49 can be used only for multiplying a rapidly alternating force (applied to end $A$ ).

Imagine a very rapidly alternating force applied to end $A$ of the lever in Fig. 49. The
A steady electromotive force would eventually produce an indefinitely ' large current through the primary coil of a transformer (resistance of coil assumed to be very small). Therefore the transformer can be used only for multiplying a rapidly alternating electromotive force (applied to the primary coil).

An alternating electromotive force is applied to the primary coil $A$ of a step-up trans- 
end $A$ will move with large back and forth velocity, and the end $B$ will move with small back and forth velocity; but end $B$ will exert a large force.

In fact, if we ignore the slight movement of the heavy body, we will have:

$$
\frac{e^{\prime}}{e^{\prime \prime}}=\frac{l^{\prime \prime}}{l^{\prime}}
$$

and

$$
\frac{i^{\prime}}{i^{\prime \prime}}=\frac{l^{\prime}}{l^{\prime \prime}}
$$

where, at any instant, $e^{\prime}$ is the force exerted on end $A$, $i^{\prime}$ is the velocity of end $A$, $e^{\prime \prime}$ is the force exerted by end $B$, and $i^{\prime \prime}$ is the velocity of end $B$. former. Then a large current flows back and forth through the primary coil and a small current flows back and forth through the secondary coil; but the secondary coil acts upon the receiving circuit with a large electromotive force.

In fact, if we ignore the small current through the primary coil which magnetizes the iron core, we will have:

$$
\frac{e^{\prime}}{e^{\prime \prime}}=\frac{Z^{\prime}}{Z^{\prime \prime}}
$$

and

$$
\frac{i^{\prime}}{i^{\prime \prime}}=\frac{Z^{\prime \prime}}{Z^{\prime}}
$$

as explained in part in Art. 30: where, at any instant, $e^{\prime}$ is the electromotive force acting on the primary coil, $i^{\prime}$ is the current flowing through the primary coil, $e^{\prime \prime}$ is the electromotive force induced in the secondary coil, and $i^{\prime \prime}$ is the current flowing through the secondary coil.

The analogy above outlined is complete, but it is not worth while to develop it in full. Thus the movement of the massive body $M$ is exactly analogous to the magnetizing current in a transformer, and the mass of the lever itself leads to behavior which is exactly analogous to magnetic leakage in a transformer. This latter is indeed developed in Art. 43; but from a point of view somewhat different from the usual discussion* of magnetic leakage in transformer theory.

* See Chapter XI, Elements of Electrical Engineering, Vol. II., Franklin and Esty, The Macmillan Co., I908. 
4I. Definition of mutual inductance.-Consider two adjacent circuits, and let one circuit be called the primary circuit and the other the secondary circuit to distinguish them. Let $i_{1}$ be the current in the primary circuit, and let $i_{2}$ be the current in the secondary circuit.

Let $\Phi_{2}$ be the magnetic flux* through the secondary circuit due to a current of $i_{1}$ abamperes in the primary circuit. It is evident from Art. I 8 that $\Phi_{2}$ is proportional to $i_{1}$. Therefore we may write:

$$
\Phi_{2}=M i_{1}
$$

in which the proportionality factor $M$ is called the coefficient of mutual induction or simply the mutual inductance of the two circuits.

Similarly, we have:

$$
\Phi_{1}=M i_{2}
$$

It is shown in the next article that the coefficient $M$ has identically the same value in equations (I) and (2).

If $i_{1}$ changes it is evident that $\Phi_{2}$ will change $M$ times as fast as $i_{1}$, because it is always $M$ times as large as $i_{1}$ according to equation (I). Therefore $\frac{d \Phi_{2}}{d t}=M \frac{d i_{1}}{d t}$. But $e_{2}=-\frac{d \Phi_{2}}{d t}$ according to Arts. 29 and 3I. Therefore we have:

$$
e_{2}=-M \frac{d i_{1}}{d t}
$$

where $e_{2}$ is the electromotive force induced in the secondary circuit by the changing value of the current in the primary circuit.

Similarly we may show that:

$$
e_{1}=-M \frac{d i_{2}}{d t}
$$

42. Kinetic energy of two circuits.-The kinetic energy of two circuits is given by the equation:

$$
W=\frac{1}{2} L_{1} i_{1}^{2}+M i_{1} i_{2}+\frac{1}{2} L_{2} i_{2}^{2}
$$

* Each part of the flux being counted $n$ times, where $n$ is the number of turns of wire encircling that part of the flux. 
in whicin $L_{1}$ is the inductance of the primary circuit and $L_{2}$ is the inductance of the secondary circuit as defined in Art. 3I, $i_{1}$ is the current in the primary circuit, $i_{2}$ is the current in the secondary circuit, and $M$ is the mutual inductance of the two circuits.

To establish equation (I) let us assume both circuits to have zero resistance for the sake of simplicity of statement, and let us consider $(a)$ the amount of work required to increase the primary current from zero to a given value $i_{1}$, with the secondary circuit open; (b) the amount of work (done on the primary circuit) to keep the primary current $i_{1}$ constant while the secondary current is being increased uniformly from zero to a given value $i_{2}$ in $t$ seconds; and $(c)$ the amount of work (done on the secondary circuit) to increase the secondary current from zero to the given value $i_{2}$.

The amount of work (a) is equal to $\frac{1}{2} L_{1} i_{1}{ }^{2}$ according to Art. 35 .

To find the amount of work $(b)$ assume that the secondary current is increased at the constant rate $\frac{i_{2}}{t}$ so that an electromotive force equal to $-M \frac{d i_{2}}{d t}=-\frac{M i_{2}}{t}$ is induced in the primary coil, according to equation (4) of Art. 4I. Therefore to keep $i_{1}$ constant, an electromotive force equal to $\frac{M i_{2}}{t}$ must act on the primary coil for $t$ seconds doing work at the rate $\frac{M i_{2}}{t} \times i_{1}$. Therefore the amount of work done will be $\left(\frac{M i_{2}}{t} \times i_{1}\right) \times t$ which is equal to $M i_{1} i_{2}$.

The amount of work $(c)$ is equal to $\frac{1}{2} L_{2} i_{2}{ }^{2}$ according to Art. 35 . The argument of Art. 35 applies to the calculation of the amount of work done on the secondary coil while the secondary current is increased from zero to $i_{2}$ and while the primary current is kept at a constant value, because the only electromotive force induced in the secondary coil is the self-induced electromotive force due to the increasing secondary circuit. 
The total work done to establish the two currents $i_{1}$ and $i_{2}$ is therefore equal to $\frac{1}{2} L_{1} i_{1}{ }^{2}+M i_{1} i_{2}+\frac{1}{2} L_{2} i_{2}{ }^{2}$, and the kinetic energy of the two circuits is equal to the total work done.

Note.-Imagine the current $i_{2}$ to be established first and $i_{1}$ afterwards, then another expression for the kinetic energy would be obtained. The only difference being that in one case the expression for $W$ contains the $M$ in equation (4) of Art. 4I whereas in the other case the expression for $W$ contains the $M$ in equation (3) of Art. 4I. But the two expressions for $W$ must be the same, therefore the two $M$ 's are identical.

43. Mechanical analog of the transformer again considered.The mass of the lever $A B$. in Fig. 49 was neglected in the discussion of Art. 40, but Fig. 50 shows a lever with heavy balls at its two ends and an attached mass $C$ at its center, the two lever arms $l$ and $l$ being assumed to be equal for the sake of simplicity. The velocity of $A$ is $i_{1}$, the velocity of $B$ is $i_{2}$, and

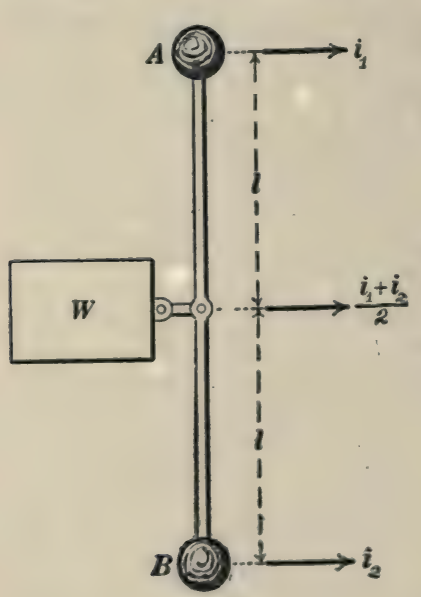

Fig. 50 . the velocity of $C$ is $\frac{i_{1}+i_{2}}{2}$; and the kinetic energy of the system is:

$$
W=\frac{1}{2} L_{1} i_{1}^{2}+M i_{1} i_{2}+\frac{1}{2} L_{2} i_{2}^{2}
$$

where, as may be easily shown:

$$
\begin{gathered}
L_{1}=A+\frac{C}{4} \\
L_{2}=B+\frac{C}{4} \\
M=\frac{C}{4}
\end{gathered}
$$

Equation (I) is identical to equation (I) of Art. 42, and the mechanism of Fig. 50 is completely analogous to two inductively related or "coupled" circuits.

44. Coupled circuits containing capacity.-A common arrangement of apparatus at the sending station for wireless telegraphy 
is shown in Fig. 5I. The condenser $C$ is charged from a high voltage supply, $a b$, until the spark gap breaks down. Then the charge on the condenser surges back and forth through the circuit $C P$. The coil $P$ is the primary of a transformer of which the secondary is the coil $S$; the back and forth surging of current through the primary circuit $C P$ induces an alternating electromotive force in the secondary coil $S$; and this induced electromotive force causes current to surge up and down in the antena. The energy is then largely radiated from the antena in the form of electric waves. Figure 52 shows a similar arrangement in

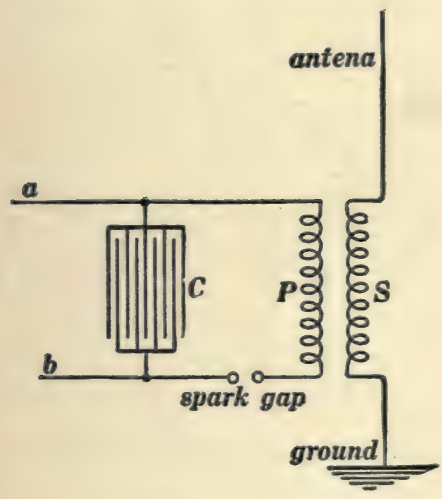

Fig. 51.

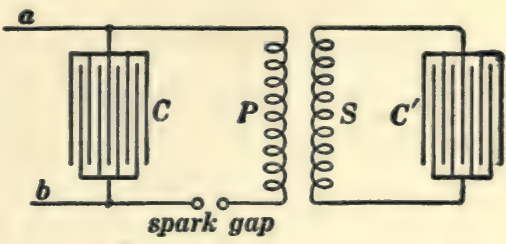

Fig. 52.

which there is no appreciable radiation from the secondary circuit $S C^{\prime}$. The two circuits $C P$ and $S C^{\prime}$ are said to be coupled through the transformer $P S$.

A mechanical arrangement which is completely analogous to Fig. 52 is shown in Fig. 53 in which the lever, $l l^{\prime}$, and the weight $W$ correspond to the transformer in Fig. 52 ; and the springs $C$ and $C^{\prime}$ correspond to the condensers in Fig. 52 .

The details of behavior of the coupled circuits in Fig. 52 can be strikingly demonstrated with the help of the arrangement in Fig. 53 as follows. The primary system $P C$ has a definite frequency of oscillation when the car $S$ is held fast, and the secondary system $S C^{\prime}$ has a definite frequency of oscillation 
when the car $P$ is held fast. When these two frequencies are adjusted to equality, the primary and secondary systems are said to be tuned to each other.

The ratio of transformation of the transformer in Fig. 52 is represented by the ratio of the two lever arms $l$ and $l^{\prime}$.

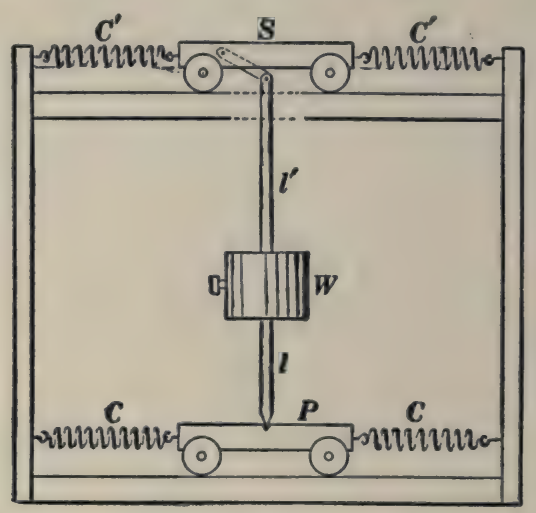

Fig. 53.

When the weight $W$ is very large or when the weights of the cars $P$ and $S$ are small, we have what is called close coupling. When the weights of the cars $P$ and $S$ are large, or when the weight $W$ is small, we have what is called loose coupling.

(a) When the primary system $P C$ is set oscillating, a series of force impulses is transmitted to the secondary system $S C^{\prime}$ by the lever, and the secondary system is quickly set into oscillation.

(b) When the primary and secondary systems are in tune with each other, the whole of the energy of the oscillating primary system is transformed into the secondary system, and the primary system comes momentarily to rest. Then the energy is transformed back from secondary to primary and so on repeatedly.

(c) When the primary and secondary systems are not in tune with each other, a definite fractional part only of the energy of the primary system is transformed to the secondary. This 
fraction depends upon the closeness of coupling and upon the closeness of tuning of the primary and secondary systems.*

\section{PROBLEMS.}

I. A room 6 meters long by 5 meters wide by 3 meters high has its longest dimension magnetic north and south. The intensity of the earth's field in the room is 0.62 gauss and the dip is $72^{\circ}$. Find the number of lines of magnetic flux across each of the walls, the ceiling, and floor of the room, and specify in each case whether the flux is passing out of the room or into the room. Ans. East and west walls, zero; north wall, 28,740 maxwells out; south wall, 28,740 maxwells in; ceiling, I76,900 maxwells in; floor, I76,900 maxwells out.

2. The pole face of the field magnet of a dynamo has an area 20 centimeters by 30 centimeters. The magnetic field between the pole face and the armature core is perpendicular to the pole face at each point and its intensity is 6,000 gausses. Calculate the number of lines of force which pass from the pole face into the armature core. Ans. 3,600,000 maxwells.

3. A slim magnet 0.25 centimeter thick and 0.5 centimeter wide is magnetized to an intensity of $\mathrm{I}, 000$ units pole per square centimeter of sectional area. Find the number of lines of magnetic flux which emanate from the north pole of this magnet. Ans. I57 I maxwells or lines.

4. A very long steel ribbon of which the thickness is o.I centimeter is magnetized so that one edge becomes a long north pole and the other edge becomes a long south pole, as shown in Fig. $p 4$, the intensity of magnetization being 800 units pole for each square centimeter of section of the steel ( 80 units pole for each centimeter length of edge). Find the intensity of the magnetic

* The mathematical theory of the electrical system in Fig. 52 and of the mechanical system in Fig. 53 is identical. This theory is developed in a paper by Louis Cohen, Bulletin of Bureau of Standards, Vol. 5, pages 5II-54I. 
field due to the north polar edge of the strip at a point distant I8 centimeters from the edge, and specifyi ts direction. Ans. 8.89 gausses directed away from the edge.

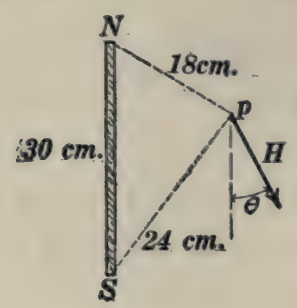

end view

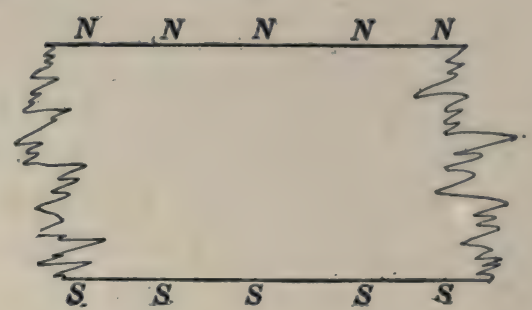

side view

Fig. p4.

Note.-The magnetic field intensity at the point $p$ due to the north polar edge of the ribbon magnet may be found as follows: Imagine a cylinder 18 centimeters in radius with its axis along the north polar edge. Let $h$ be the field intensity at the surface of this cylinder due to the north polar edge of the ribbon magnet. Then $h$ is at right angles to the cylindrical surface, and the magnetic flux through one centimeter of length of the cylindrical surface can be easily found in terms of $h$; and this flux must be equal to $4 \pi$ times the number of units pole on one centimeter of length of the north polar edge of the magnet.

5. A vertical wire 3 meters long is moved sidewise, towards magnetic east or west, at a velocity of 25 meters per second. Find the electromotive force induced in the wire in volts, the horizontal component of the earth's field being 0.18 gauss. Ans. 0.00135 volt.

6. The pole face of a dynamo is 30 centimeters long in the direction parallel to the axis of the armature, and the field intensity in the gap space between the pole face and the armature core is 6,000 gausses. The wires on the armature are 12 centimeters from the axis of the armature, and the speed of the armature is $\mathrm{I}, 800$ revolutions per minute. Find the electromotive force in volts induced in each armature wire (30 centimeters in length) as it passes across the pole face. Ans. 4.07 volts. 
7. The core of an induction coil carries 100,000 lines of magnetic flux when current is flowing through the primary coil. When the primary circuit is broken the flux in the core drops to Io,000 lines in 0.003 second. How many turns of wire are required in the secondary coil in order that an average electromotive force of 25,000 volts may be induced in this coil during the 0.003 second? Ans. 83,333 turns.

8. The ring armature of a direct-current bipolar dynamo has 260 turns of wire upon it, the armature is driven at a speed of I,200 revolutions per minute, and the magnetic flux from a pole face into the armature core is 2,200,000 lines. Calculate the electromotive force of the dynamo.in volts. Ans. II4.4 volts.

9. A circular metal disk $D D$ is Io centimeters in radius and is mounted on an axle in a long hollow coil SS having 5 turns of wire per centimeter of length as shown in Fig. p9. Two brushes maintain contact with center and edge of the disk, and the galvanometer gives no deflection when the disk runs at a speed of 75.2 revolutions per second. What is the resistance of the coil $R$ ? Ans. I, 485,000 abohms.

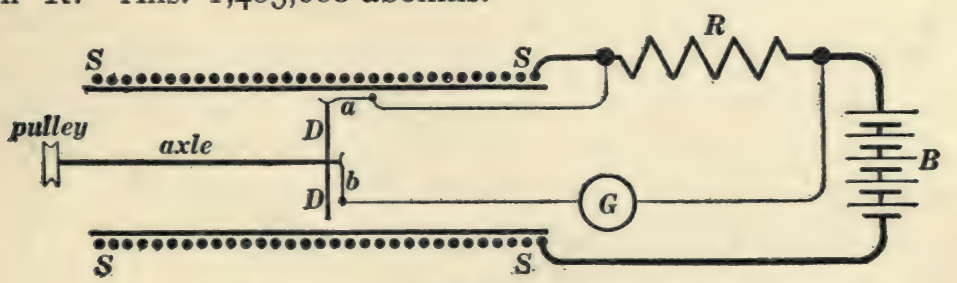

Fig. p9.

Note.-Find intensity of the magnetic field in the very long coil SS for an assumed current $I$ in the coil. Then find the magnetic flux through the disk $D D$. This amount of flux is cut per revolution by every radius of the disk, and multiplying by the revolutions per second gives the electromotive force between the brushes; and this electromotive force balances the electromotive force across the resistance $R$.

The arrangement shown in Fig. p9 was devised by L. Lorenz in 1873 for the "absolute" measurement of resistance; that is, for the measurement of resistance directly in mechanical terms.

Io. A transformer takes alternating current from supply mains at I,IOO volts and delivers current to service mains at IIO volts. 
The primary coil of the transformer has 560 turns of wire. How many turns of wire are there in the secondary coil? The transformer delivers 250 amperes to the service mains. How much current does it take from the supply mains? A usual allowance in transformer coils is $\mathrm{I}, 000$ circular mils sectional area of wire for each ampere. Find size of wire used in primary coil and in secondary coil of the transformer. Ans. 56 turns, 25 amperes, 25,000 circular mils, 250,000 circular mils.

II. Let it be assumed that the force required to propel a canal boat is proportional to the velocity of the boat. A force of 50 pounds is required to maintain a steady velocity of 5 feet per second. (a) Find the value of the coefficient by which the velocity of the boat must be multiplied to give the frictional drag, and specify the unit in terms of which this coefficient is expressed. (b) Find the velocity at which the boat would be propelled by a force of 36 pounds. (c) Find the rate at which work is done by a force of 36 pounds in propelling the boat. Ans. (a) Io pounds per foot-per-second. (b) 3.6 feet per second. (c) I29.6 foot-pounds per second.

I2. When a force of 50 pounds is applied to the above canal boat, the boat starts from rest and after some time reaches its full speed of 5 feet per second. At a given instant the velocity of the boat is 3 feet per second. At this instant: (a) Find the rate at which work is done on the boat by the propelling force. (b) Find the dragging force which is acting on the boat due to the friction of the water. (c) Find the rate at which work is dissipated in overcoming the friction of the water. (d) Explain what is becoming of the difference between $(a)$ and $(c)$. Ans. (a) 150 foot-pounds per second. (b) 30 pounds. (c) 90 foot-pounds per second. (d) It is being stored as increasing kinetic energy of the boat.

I3. An electromotive force of 50 volts acts on a circuit of which the resistance is 10 ohms. At a certain instant during the time that the current is growing from zero to its full value, the current has an actual value of 3 amperes. At this instant: $(a)$ Find the 
rate at which the generator delivers work to the circuit. Find the dragging force in volts which is opposing the flow of the current through the circuit due to the resistance of the circuit. (c) Find the rate at which work is dissipated in overcoming the resistance of the circuit. (d) Explain what is becoming of the difference between $(a)$ and $(c)$. Ans. (a) I50 watts. (b) 30 volts. (c) 90 watts. (d) It is being stored as increasing kinetic energy of the circuit.

I4. The field winding of a dynamo has 50 ohms resistance and, approximately, 7.5 henrys of inductance. Assuming that the current grows in the coil in accordance with equation (4) of Art. 36 calculate the time required for the current in the winding to reach 2 amperes, when the winding is connected to a generator of which the electromotive force is I Io volts. Ans. 0.359 second.

I5. A current has been left to die away in a circuit of $0.6 \mathrm{ohm}$ resistance and 0.05 henry inductance. Find the rate of change of the current as it passes the values Ioo amperes, Io amperes, and one ampere. Ans. - I,200 amperes per second, - I20 amperes per second, - I2 amperes per second.

I6. Find the approximate inductance in henrys of a cylindrical coil 25 centimeters long, 5 centimeters mean diameter, wound with one layer of wire containing I50 turns. Ans. 0.000222 henry.

I7. The choke coil of a lightning arrester consists of 50 turns of wire wound in one layer on a cylinder of which the diameter is I5 centimeters and the length is 50 centimeters. (a) Calculate the approximate inductance of this coil. (b) Calculate the approximate rate of increase of current in the coil at the instant that a lightning discharge jumps across two centimeters of air in preference to going through the coil. Ans. (a) o.oooIII henry, $(b)$ 360,000,000 amperes per second.

Note.-The electromotive force required to strike across 2 centimeters of air is approximately 40,000 volts.

I8. The field coil of a dynamo has 5,000 turns of wire and, when a current of one ampere flows through the field winding, 
I,500,000 lines of force are produced through the field core. Assuming that the flux is proportional to the exciting current, find the inductance of the field coil in henrys. Ans. 75 henrys.

I9. A battery having an electromotive force of Io volts and a resistance of one ohm is connected to a coil of wire wound on an iron core. The coil has I,000 turns of wire and its resistance is 4 ohms. What is the current in the coil when the magnetic flux in the core is increasing at a rate of 500,000 lines per second? Ans. I ampere.

20. A certain spool wound full of wire o.I centimeter in diameter has an inductance of 0.08 henry. The same spool is wound full of wire 0.32 centimeter in diameter? What is its inductance? Ans. 0.000762 henry.

21. A spool 5 times as large as the spool mentioned in problem 20 but similar in shape, is wound with wire 6 millimeters in diameter. What is its inductance? Ans. 0.193 henry.

22. A transmission line consists of two No. I B. \& S. wires at a distance of 2 feet apart center to center. Find the inductance in henrys of ten miles of this line. Ans. 0.0329 henry. 
All quantities in this chapter are expressed in "electromagnetic" c.g.s. units: magnetic field intensity in gausses, magnetic flux in lines (maxwells), current in abamperes, length in centimeters, area in square centimeters, etc.

\section{CHAPTER IV.}

\section{ELECTROMAGNETS. MAGNETISM OF IRON.}

45. Bunched windings and distributed windings.-The electromagnet, such as is used for the field magnet of a dynamo, consists of a rod of iron surrounded by a winding of insulated copper wire, through which an electric current flows. The iron rod is called the core of the electromagnet, and the electric current which flows through the winding of wire is called the exciting current. The exciting current produces a magnetic field in the region occupied by the iron core, and the effect of this magnetizing field, as it is called, is to magnetize the core and cause a certain amount of magnetic flux to "flow" through it. The iron rod or core usually forms a complete or nearly complete circuit, called a magnetic circuit, through which the magnetic flux "flows."

Two distinct cases occur in the arrangement of the windings of wire upon the iron core as follows:

(a) Uniformly distributed winding.-The winding of wire may be distributed uniformly along the entire length of the iron rod or core. In this case the magnetic field which the winding produces in the region occupied by the iron core has sensibly the same value at every point in the rod and is sensibly parallel to the rod at each point. The magnetizing action of such a field upon an iron rod depends simply upon the intensity of the field. Electromagnets having uniformly distributed windings are seldom used in practice except in the magnetic testing of iron, for which purpose the iron to be tested is usually made into a test ring, ${ }^{*}$ which is wound uniformly with wire.

* This applies to the ballistic method of testing iron, which is due to Rowland. In Ewing's method the test piece of iron is in the form of a long slim rod. 
(b) Bunched winding.-The winding of wire may be bunched at one or more places along the iron core. In this case the magnetic field which the winding produces in the region occupied by the iron core varies greatly in intensity from point to point in the core, and the direction of this field is not parallel to the rod at each point. The magnetizing action of such a field upon an iron rod is discussed in the next article.

Electromagnets usually have bunched windings.

46. The magnetizing action of a bunched winding. Definition of magnetomotive force.-The magnetizing action upon an iron rod of a non-uniform magnetic field, such as the magnetic field produced by a bunched winding, depends upon the average value (along the rod) of the component* of the magnetizing field parallel to the rod. The product of this average value and the length of the rod is called the magnetomotive force along the rod. That is:

$$
\mathscr{F}=l A
$$

in which $\mathcal{F}$ is the magnetomotive force along a rod in c.g.s. units, $l$ is the length of the rod in centimeters, and $A$ is the average value, along the rod, of the component of the magnetizing field parallel to the rod. The rod may be straight or curved.

A rod passing through a magnetic field determines a certain line or path through the field, and we speak of the magnetomotive force along this path, whether the rod is there or not.

47. Proposition. - The magnetomotive force along a path in a magnetic field is equal to the work per unit pole done by the magnetic field upon a magnet pole while the pole is made to travel along the path; that is, the magnetomotive force along a path is equal to $W / m$, where $W$ is the work done by the field upon a pole of strength $m$ while the pole is made to travel along the path. That is:

$$
\mathscr{F}=\frac{W}{m}
$$

in which $\mathscr{F}$ is the magnetomotive force along the path.

* At each point. 
Procf.-The product $m A$ is the average value along the path of the component parallel to the path of the force $f(=m \mathscr{r})$ with which the field acts on the pole, the meaning of $A$ being explained in Art. 46. Therefore $\operatorname{lm} A$ is the work done on the pole by the field as the pole travels from one end of the path to the other. That is:

$$
W=\operatorname{lm} A
$$

Dividing both members of this equation by $m$, and remembering that $l A=\mathcal{F}$ according to equation (I), Art. 46, we have equation (I) of this article.

The above proposition is usually taken as the definition of magnetomotive force along a path, and from this definition it may be shown that the magnetomotive force along a path is equal to what is called the line integral* of the magnetic field along the path, as follows: Consider an element $\Delta l$ of a path $p p^{\prime}$, Fig. 54. 'Let $\mathscr{H}$ represent the intensity of the magnetic field at this element and $\epsilon$ the angle between $\mathscr{H}$ and $\Delta l$. Then the component of $\mathscr{H}$ parallel to $\Delta l$ is $\mathscr{H} \cdot \cos \epsilon$.

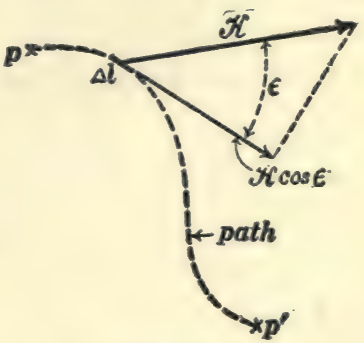

Fig. 54.

Let a magnet test pole of strength $m$ be moved along $\Delta l$. The force with which the magnetic field acts upon this pole is $m \mathscr{H}$, and the component of this force parallel to $\Delta l$ is $m \mathscr{H} \cos \epsilon$, so that $m \mathscr{F}^{\prime} \cos \epsilon \times \Delta l$ is the work $\Delta W$ done by the field on the pole as the pole moves along $\Delta l$. That is:

$$
\Delta W=m \mathscr{H} \cos \epsilon \cdot \Delta l
$$

from which, by integration, we have:

$$
\frac{W}{m}=\mathscr{F}=\int \mathscr{H} \cos \epsilon \cdot d l
$$

* A very simple and complete discussion of line and surface integrals such as are used in electromagnetic theory is given in Chapter IX of Franklin, MacNutt and Charles's Calculus, South Bethlehem, $\mathrm{Pa}$, rgr3. 
48. Magnetomotive force of a coil.-The most important case of magnetization of an iron rod or core by a bunched winding

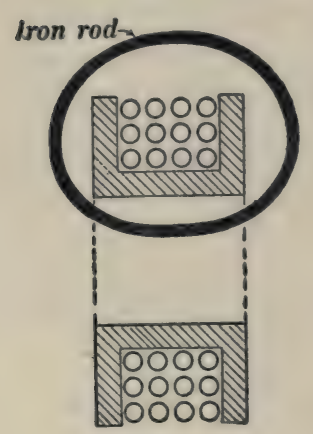

Fig. 55. is the case in which the core is in the form of an endless rod which passes through, or links with, the coil as shown in Fig. 55. The magnetomotive force along the rod in this case is entirely independent of the shape and length of the rod and of the shape and size of the coil. It depends only upon the number of turns of wire in the coil and upon the strength of the current in the wire. This definite magnetomotive force along an endless rod which links with a coil of wire in which an electric current is flowing, is called the magnetomotive force of the coil.

Examples.-Fig. 56 shows a two-pole dynamo with two field coils $Z Z$. The field-magnet iron of this dynamo and the iron of the armature together constitute a nearly endless rod or core of iron which links with both field coils, and which constitutes what is called the magnetic circuit of the dynamo.

Figure 57 is a sketch of a four-pole dynamo with four field coils $Z Z Z Z$. The iron of the field magnet and armature presents in this case four distinct

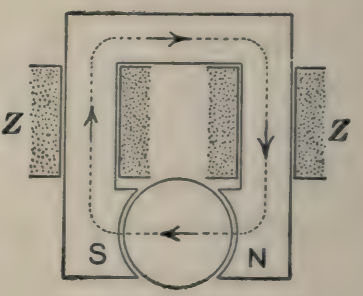

Fig. 56. magnetic circuits, as indicated by the dotted lines. Through each of these magnetic circuits, flux is forced by the combined magnetomotive forces of the two field coils through which the magnetic circuit passes, that is, with which it links.

The total magnetomotive force acting on any magnetic circuit is equal to the sum of the magnetomotive forces of the coils with which the circuit links.

49. Proposition.-The magnetomotive force of a coil is given by the equation: 


$$
\mathcal{F}=4 \pi Z i
$$

in which $Z$ is the number of turns of wire in the coil, and $i$ is the strength of the current in the wire in abamperes. When the current is expressed in amperes we have:

$$
\mathscr{F}=\frac{4 \pi}{10} Z i
$$

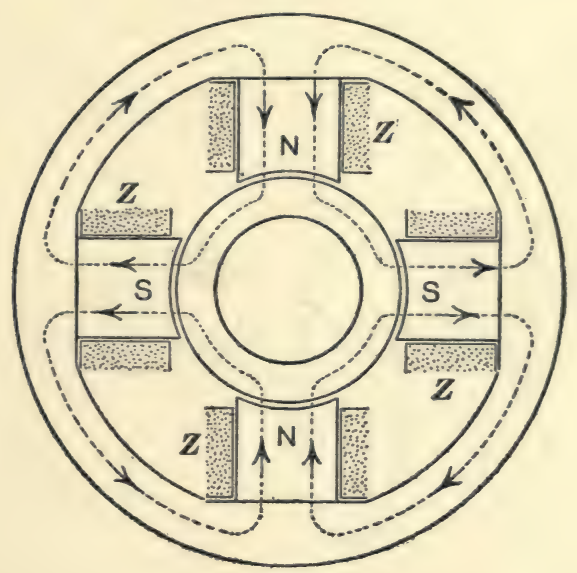

Fig. 57.

Proof of equation (I) above.-Before proceeding to the derivation of equation (I), it is necessary to find an expression for the total work $W$ done in keeping the current $i$ in a coil constant while the magnetic flux through the opening of the coil is increased by a specified amount $\Phi ; W$ being expressed in ergs, $i$ in abamperes, and $\Phi$ in maxwells or lines. Now, while the flux is increasing, a back electromotive force equal to $Z \frac{d \Phi}{d t}$ abvolts is induced in the coil, and therefore (assuming the coil to have zero resistance for the sake of simplicity of statement) an electromotive force $e=Z \frac{d \Phi}{d t}$ will have to act on the coil to keep the back electromotive force from decreasing the current. Consequently work will have to be done on the coil at the rate 
$e i$, or $Z \frac{d \Phi}{d t} \times i$, to keep the current from increasing. That is:

$$
\frac{d W}{d t}=Z i \cdot \frac{d \Phi}{d t}
$$

But $Z i$ is a constant, because $i$ is being kept from changing. Therefore by integrating (3) from $W=0$ and $\Phi=0$ we get:

$$
W=Z i \Phi
$$

in which $W$ is the work in ergs done to keep a current of $i$ abamperes constant in a coil (of zero resistance) while the mag-

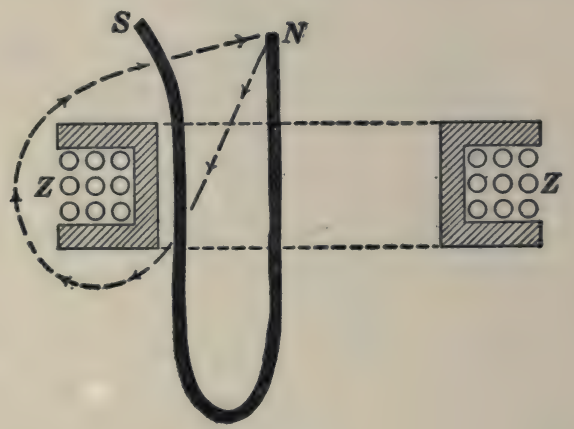

Fig. 58.

netic flux through the opening of the coil increases by the amount of $\Phi$ maxwells, and $Z$ is the number of turns of wire in the coil.

We shall now proceed to the proof of equation (I). Let $Z Z$, Fig. 58, represent a coil of $Z$ turns of wire. Imagine $N S$ to be a flexible magnet. Let the north pole of this flexible magnet

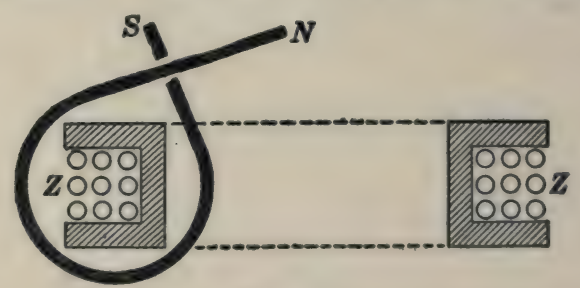

Fig. 59.

be carried through the coil and around to its initial position along the dotted path. The flexible magnet will then link with the 
coil of wire as shown in Fig. 59. Let $\mathscr{F}$ be the magnetomotive force along the dotted path, and let $m$ be the strength of the pole $N$ which has been carried around the path. Then, according to Art. $47, \mathcal{F}_{m}$ is the work done on the pole by the magnetic field of the coil. This work done on the moving pole by the magnetic field of the coil is the work which is spent in keeping the current constant in spite of the electromotive force induced in the coil by the moring pole. This is evident when we consider that work done on the pole by the field must be made good, that is, energy must be supplied from somewhere; and when we consider that the energy stored in the system is the same in Figs. $5^{8}$ and 59. Therefore, the only possible source of the energy for doing work on the moving pole is the work that is done to keep the current in the coil constant.

Now the two poles of the flexible magnet are in the same positions before and after the movement; therefore, of the total number of lines of force which radiate from these poles, the same number pass through the coil before and after the movement. On the other hand, the flux $4 \pi m$ (see Art. 26), which passes along the magnet from pole to pole, passes through the coil after the movement, so that the flux through the opening of the coil is increased by the amount $4 \pi m$ by the movement of the pole. Therefore, according to equation (4), $Z i \times 4 \pi m$ is the work spent in keeping the current constant during the movement of the pole and, since this is equal to the work $\mathscr{I}_{m}$ done upon the pole by the magnetic field of the coil, we have

or

$$
\mathscr{J F}_{m}=4 \pi i Z m
$$

$$
\mathscr{F}=4 \pi Z i
$$

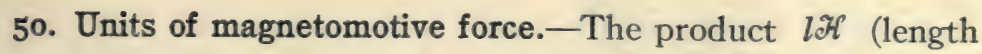
of path in centimeters multiplied by intensity of magnetic field in gausses) gives the magnetomotive force along the path (when $l$ and $\mathscr{H}$ are parallel, of course) in c.g.s. units. The name gilbert has been adopted by the American Institute of Electrical Engineers for the c.g.s. unit of magnetomotive force. 
The magnetomotive force along a path which links with one turn of wire carrying one ampere of current is called one ampereturn. The magnetomotive force of any coil, in ampere-turns, is equal to the product of the current flowing in the coil in amperes and the number of turns of wire in the coil. In magnetic calculations it is usually convenient to reduce ampere-turns of magnetomotive force to c.g.s. units (gilberts), which is done by multiplying ampere-turns by $4 \pi / 10$, according to equation (2) of Art. 49.

The product, field intensity multiplied by length of path, gives magnetomotive force, so that the quotient obtained by dividing magnetomotive force by length of path is field intensity. When a magnetomotive force which is expressed in ampere-turns is divided by length of path, we have a magnetic field intensity expressed in ampere-turns-per-centimeter, or in ampere-turns-perinch, as the case may be.

51. Magnetizing force in iron.-When an iron rod is placed in a magnetic field and is magnetized thereby, the actual magnetic field along the rod depends not only upon the original cause of the field but also upon the newly created magnetic poles of the rod itself. Thus when an iron rod is placed in a coil of wire through which an electric current is flowing, the field along the rod is due to the combined action of the coil and the poles of the rod.

The magnetizing force at a given point in an iron rod is defined as the net resultant field intensity $\mathscr{H}$ at the point due to the combined action of the external original cause and the newly created magnetic poles of the rod. The influence of the poles of a magnetized rod is always such as to reduce the intensity of the original field along the rod.

When the iron rod is slim, its poles are weak, and if the rod is long, its poles are at considerable distances from the middle portions of the rod. In this case, therefore, the poles of the rod do not produce any appreciable weakening of the original field along the middle portions of the rod. When the iron rod is in 
the form of a ring which is magnetized by a winding of wire distributed around the ring, then the rod has no poles and consequently no demagnetizing action on itself. In the case of a closed iron ring and in the case of a very long slim rod, the magnetic field produced by the winding of wire is the net actual field which magnetizes the rod.

Example.-An iron rod 20 centimeters long is placed in a magnetic field, which, but for the presence of the rod, would be a uniform magnetic field of 60 units intensity, the rod being parallel to the direction of this field. Let us suppose that the rod becomes magnetized to such a degree that the strengths of the poles at the ends of the rod are $+2,700$ units, and $-2,700$ units respectively. In this case a point near the middle of the rod is approximately Io centimeters distant from each of the poles, and the actual field $\mathscr{H}$ near the middle of the rod may be thought of as made up of three parts, namely, $(a)$ the original field of 60 units intensity which is directed towards the $N$ pole of the rod, (b) the field of $2700 / \mathrm{IO}^{2}$ units intensity due to the $N$ pole of the rod and directed towards the $S$ pole, and $(c)$ the field of $2,700 / \mathrm{IO}^{2}$ units intensity due to the $S$ pole of the rod and directed towards the $S$ pole. ${ }^{*}$ Therefore the net result is a field of which the intensity near the middle of the rod is 60 minus 27 minus 27 units, or 6 units.

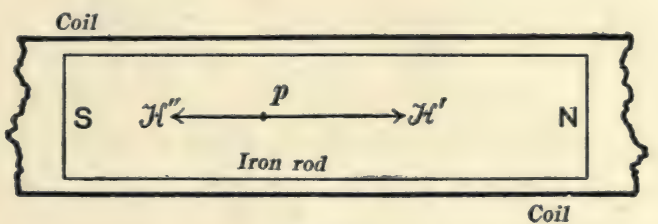

Fig. 60.

A clear idea of the significance of magnetizing force at a point in iron, as above defined, may be obtained as follows: Figure 60 shows an iron rod inside of a long coil of wire. The magnetizing field at the point $p$ is defined as the resultant field which would

* See Art. 9. 
be produced at $p$ by the combined action of the coil and of the poles $N$ and $S$ of the rod. The field which would be produced at $p$ by the coil alone is represented by the arrow $\mathscr{H}^{\prime}$, the field which would be produced at $p$ by the poles $N$ and $S$ alone is represented by the arrow $\mathscr{H}^{\prime \prime}$, and the net magnetizing field at $p$ is the resultant of $\mathscr{H}^{\prime}$ and $\mathscr{H F}^{\prime \prime}$.

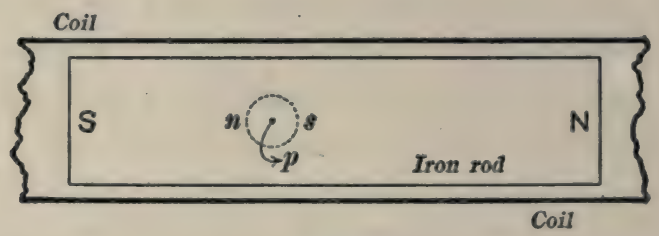

Fig. 61.

The fundamental definition of the intensity of a magnetic field at a point as the force in dynes which would act upon a unit test pole placed at the point, has no meaning unless it is possible to place the test pole at the point, that is to say, the point must be in air. But if a cavity is made around the point $p$ as shown by the dotted circle in Fig. 6I, the walls of this cavity will be

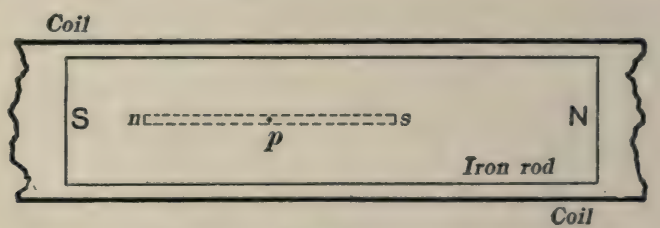

Fig. 62.

magnet poles as indicated by the letters $n$ and $s$, and the field intensity in this cavity will not be that which is due merely to the combined action of the coil and poles $N$ and $S$ at the ends of the rod. If, however, the cavity around $p$ is very long and narrow, as shown in Fig. 62, then the poles $n$ and $s$ at the ends of this cavity are weak and far away from $p$, so that these poles $n$ and $s$ do not produce at $p$ any perceptible field, and therefore the field intensity in the narrow longitudinal cavity shown in Fig. 62 is the resultant of $\mathscr{H F}^{\prime}$ and $\mathscr{H}^{\prime \prime}$ of Fig. 60. That is, the 
field intensity in a narrow longitudinal* cavity is the actual value of the magnetizing field in the iron.

In the magnetic testing of iron, the iron sample is always a very long slim rod or a closed ring so that the sample when magnetized does not have any demagnetizing action upon itself. That is to say, the magnetic field due to the magnetizing coil is the net magnetizing field.

Also, in transformers, the magnetic circuit is nearly all of iron (without any free magnet poles), and therefore the magnetizing action of the windings is not to any perceptible extent complicated by demagnetizing action of the iron core upon itself.

The above discussion (see Figs. 60, 61 and 62) is intended only to lead to a clear understanding of the term net magnetizing field; no attempt is made in this text to take account of demagnetizing action in numerical calculations. $\dagger$

52. Intensity of magnetization.-Let $m$ be the strength of each of the magnetic poles at the ends of a magnetized iron rod of which the sectional area is $s$ square centimeters. The polestrength-per-unit-sectional-area, namely $m / s$, is called the intensity of magnetization, $\mathfrak{J}$, of the rod. That is

$$
\mathfrak{d}=\frac{m}{s}
$$

53. Magnetic flux through a magnetized rod.-Consider a very long slim iron rod, $s$ square centimeters in sectional area, placed in and parallel to a magnetic field, which, but for the presence of the rod, would be a uniform magnetic field of intensity भ. Then the actual intensity of the magnetizing force along the middle portions of the rod is $\mathscr{H}$, as explained in Art. 5I. The magnetic field in the neighborhood of each end of the rod is a composite field which is the resultant of two distinct parts, $a$ and $b$; and the magnetic flux which comes up to one end of

* Parallel to the direction of magnetization of the iron.

$\dagger$ Except in a very simple way in allowing for the effect of the air gaps in the magnetic circuit of a dynamo. See Art. 57. 
the rod, flows through the rod, and passes out from the other end of the rod, consists of two distinct parts which correspond to the respective parts, $a$ and $b$, of the field near the ends of the rod.

(a) The part $a$ of the field is the original uniform field $\mathscr{H}$. The lines of force of this field are shown by the parallel straight lines in Fig. 63. The amount of magnetic flux which comes in to one end of the rod and passes out from the other end of the rod because of this uniform field $\mathscr{H}$, is $\mathscr{H}_{\mathcal{S}}$ lines.

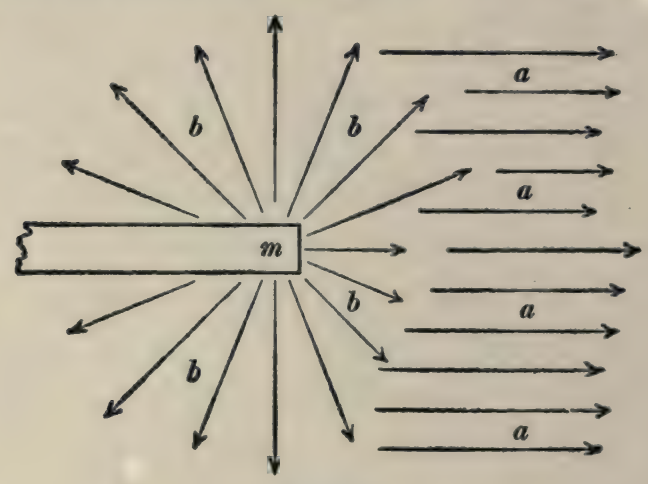

Fig. 63.

(b) The part $b$ of the field is the magnetic field due to the pole $m$ of the rod. The lines of force of this field are shown by the radiating straight lines in Fig. 63. The amount of magnetic flux which comes in to one end of the rod and passes out from the other end of the rod, because of this field, is $4 \pi m$ lines, as explained in Art. 26.

The total magnetic flux $\Phi$ coming in to one end of the rod and passing out from the other end is equal to the sum of the two parts, $a$ and $b$, so that we have:

$$
\Phi=4 \pi m+\mathscr{H} \mathcal{H}
$$

and this flux passes through the rod. If $\mathscr{H}$ is zero we have $\Phi=4 \pi m$.

Now $m=\mathfrak{I}_{s}$ from equation (I) of Art. 52, and therefore equation ( $\mathrm{I}$ ) may be written: 


$$
\Phi=4 \pi \mathfrak{d}_{s}+\mathscr{H}_{\mathcal{S}}
$$

The part $4 \pi m$ or $4 \pi \mathfrak{d} s$ of the flux through an iron rod is due to the magnetized condition of the rod, and the part $\mathscr{H} \mathcal{S}$ is due to the magnetizing force directly. This part, $\mathcal{H}_{S}$, is the amount of flux which would pass through the region occupied by the rod if the rod were removed.

54. Flux density in iron.*-The magnetic flux per unit sectional area of an iron rod, namely $\Phi / s$, is called the flux density in the rod, and it is represented by the letter $\mathfrak{B}$. That is:

$$
\mathscr{B}=\frac{\Phi}{\mathcal{S}}
$$

Substituting $\mathscr{B} s$ for $\Phi$ in equation (2) of Art. 53, we have:

$$
\mathscr{B}=4 \pi \mathfrak{d}+\mathscr{H}
$$

The flux density in an iron rod is equal to the intensity of the magnetic field in a thin crevasse cut across the rod. This is evident when we consider in the first place that flux density in air is identically the same thing as field intensity according to Art. 25, and in the second place, that a very thin crevasse does not sensibly disarrange the flux through a rod, so that the flux density in the crevasse is the same as in the rod. If the crevasse is not very narrow, a portion of the flux passes out of the rod, around the crevasse, and back into the rod again. In this case the flux density, or field intensity in the crevasse, is less than the flux density in the part of the rod which is remote from the crevasse.

55. Residual magnetism. Permanent magnets.-An iron rod retains a portion of its magnetism when it is removed from the magnetic field in which it has been magnetized, or, in case of an electromagnet, when the magnetizing current is reduced to zero. The magnetism thus left in a bar of iron (in the core of an electromagnet) is called residual magnetism. The poles of a short thick bar produce a strong demagnetizing field along the bar, and

* Frequently called magnetic induction or simply induction. 
therefore a short thick bar does not retain as much residual magnetism as a long slim bar, or as a bar which forms a closed or nearly closed magnetic circuit.

A long slim bar or a closed ring of soft annealed wrought iron or mild steel may retain as much as 90 per cent of its initial magnetism. But a very weak demagnetizing field or a slight mechanical shock is sufficient to cause the soft iron or steel to lose nearly all of its residual magnetism; cast iron and harddrawn iron wire retain a smaller portion of their magnetism, but with greater persistence; and hardened steel retains a portion of its magnetism very persistently, even when roughly handled. Magnetized bars of hardened steel are called permanent magnets.

The more persistently a sample of iron or steel retains its residual magnetism, the greater the intensity of the magnetizing force required to magnetize it. Thus hardened bars of steel are magnetized by placing them between the poles of a strong electromagnet or by placing them in a coil of wire through which a strong electric current is caused to flow.

56. Magnetization curves. - When an iron rod is acted upon by a given magnetizing force, the intensity of magnetization of the rod (and also the flux density in the rod) may have any value whatever (between certain limits), on account of the tendency of the iron to retain its previous magnetic state. Thus the intensity of magnetization of an iron rod is much greater for a given value of $\mathscr{H}$, if the given value of $\mathscr{H}$ is reached by a decrease from a higher value, than if the given value of $\mathscr{H}^{\prime}$ is reached by an increase from a lower value.

If, however, an iron rod is subjected to mechanical shocks, or if the magnetizing field pulsates slightly before it settles to a steady value, then the rod tends to settle to a state which depends only on the value of $\mathscr{H}$, that is, to a state which is independent of the previous magnetic condition of the rod. Under these conditions, therefore, definite values of $\mathfrak{B}$ and $\mathscr{J}$ are produced 
by a given value of $\mathscr{H}$. These values are called the normal values of $\mathscr{H}$ and $\mathcal{H}$ corresponding to the given value of $\mathscr{H}$. For example, a dynamo having a given exciting current in its field coils tends to settle to a state in which the magnetization of its field magnet is the normal value corresponding to the given value of its field current, because of the mechanical vibrations of the machine and because of slight variations of magnetizing action due to slight changes of field current and of armature current.

The normal $\mathscr{J}$ and $\mathscr{H}$ curve.*-The curve of which the abscissas represent values of magnetizing force $\mathscr{H}$ and of which the ordinates represent the corresponding normal values of $\mathscr{J}$ for a given sample of iron or steel is called the normal $\mathcal{J}$ and $\mathscr{H}$ curve of the sample of iron or steel. Figure 64 shows the normal $\mathfrak{D}$ and $\mathscr{H}$ curves for annealed wrought iron and for ordinary cast iron.

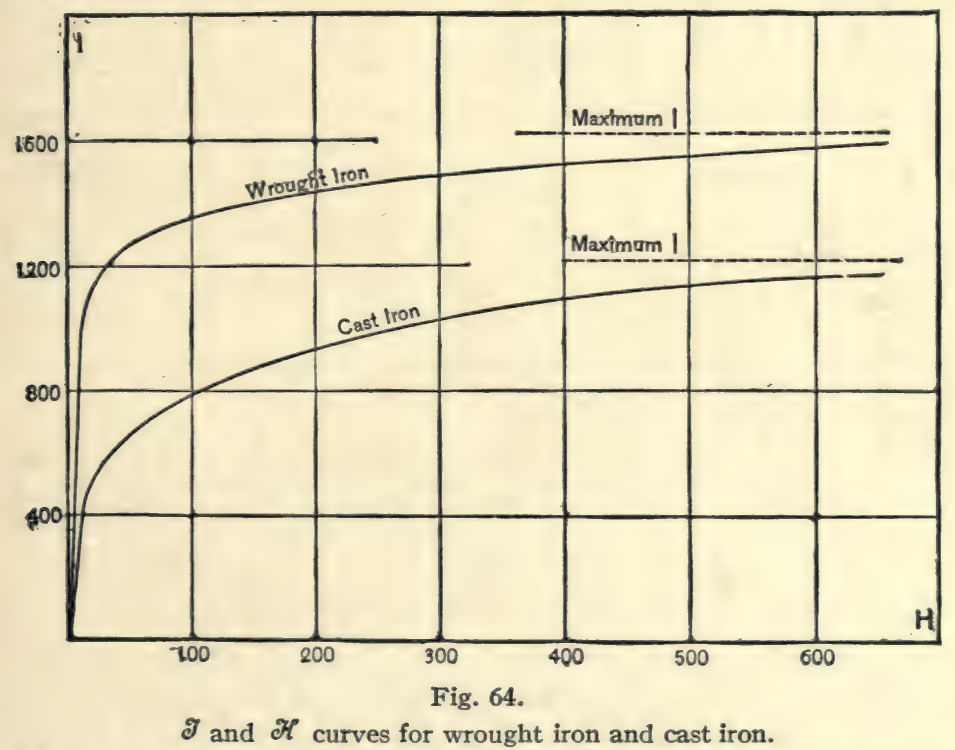

* Methods for determining these curves experimentally are explained in laboratory manuals. See especially Franklin, Crawford and MacNutt's Practical Physics, Vol. II, pages II3-I24, The Macmillan Co., New York, I908. 
The intensity of magnetization $\mathfrak{d}$ approaches a definite limiting value for a given kind of iron when $\mathscr{H}$ is increased indefinitely. This limiting or saturation value of $\mathscr{d}$ is about I,730 units pole per square centimeter for annealed wrought iron, and about I,250 units pole per square centimeter for ordinary cast iron.

The value of $\mathfrak{J}$ for a good permanent magnet made of a straight bar of hardened tungsten steel, the length of the bar being about 15 times its diameter, is about 700 or 800 units pole per square centimeter.

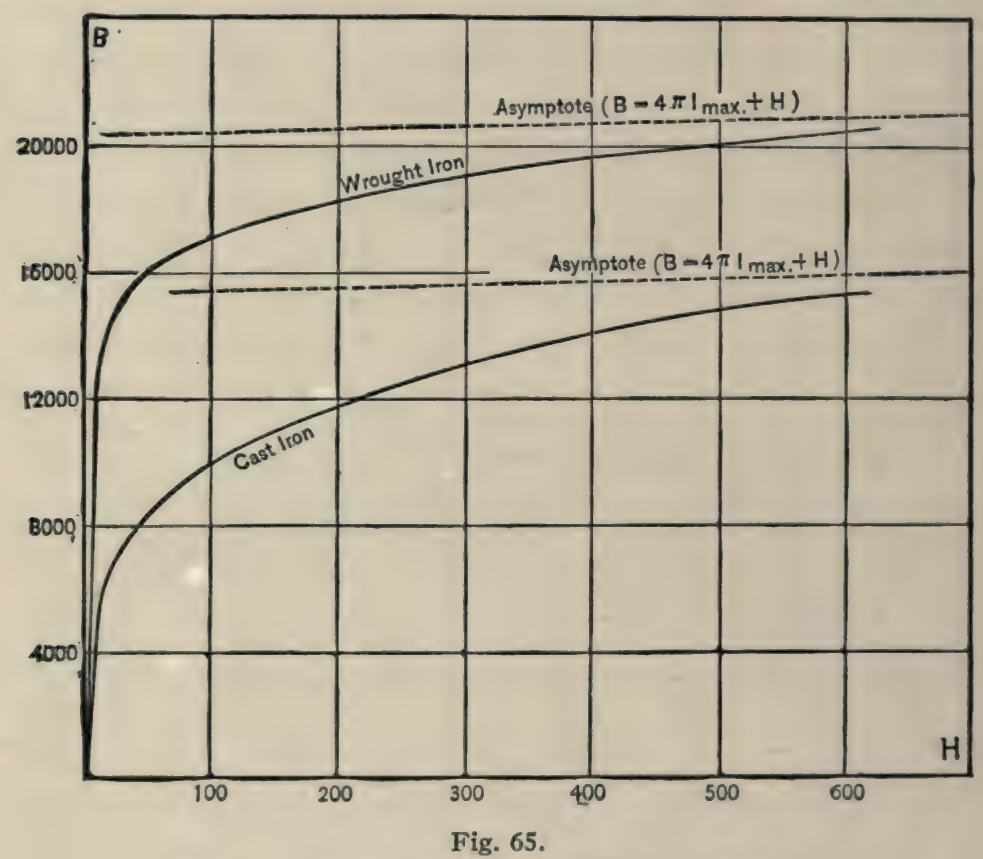

$\mathscr{B}$ and $\mathscr{H}$ curves for wrought iron and cast iron.

The normal $\mathscr{B}$ and $\mathscr{H}$ curve. The curve of which the abscissas represent values of magnetizing force $\mathscr{H}$ and of which the ordinates represent the corresponding normal values of $\mathscr{B}$ for a given kind of iron or steel is called the normal $\mathscr{B}$ and $\mathscr{H}$ curve of the iron or steel. Figure 65 shows the normal $\mathscr{B}$ and 
$\mathscr{H}$ curves for annealed wrought iron and for ordinary cast iron. The value of $\mathscr{B}$ increases indefinitely in a given sample of iron when $\mathscr{H}$ is increased indefinitely.

The sharp bend in the $\mathscr{B}$ and $\mathscr{H}$ curve is called the "knee" of the curve. The iron is approximately saturated when $\mathscr{H}$ has been increased beyond the knee of the curve, and any further increase of $\mathscr{H}$ produces only slight increase of $\mathscr{B}$. Thus a magnetizing force of ro units produces a flux density of 12,400 lines per square centimeter in a certain sample of annealed wrought iron, and a magnetizing force of double this intensity produces only 14,330 lines per square centimeter; that is, doubling the value of $\mathscr{H}$ (from Io to 20 units) produces only about I5 per cent increase of $\mathscr{B}$. Wrought iron has been subjected to a magnetizing field of about 20,000 units by Ewing who found the corresponding value of $\mathscr{B}$ to be about 40,000 lines per square centimeter, and the corresponding value of $\mathscr{I}$ to be 1,730 units pole per square centimeter.

The accompanying table gives the corresponding values of $\mathfrak{B}$ and $\mathscr{H}$ for wrought iron, for cast iron and for soft cast steel.

TABLE.

Magnetic Properties of Iron and Steel.

\begin{tabular}{|c|c|c|c|c|c|c|c|c|}
\hline \multicolumn{3}{|c|}{ Wrought Iron (Hopkinson). } & \multicolumn{3}{|c|}{$\begin{array}{l}\text { Cast Iron } \\
\text { (M. E. Thompson).* }\end{array}$} & \multicolumn{3}{|c|}{ Soft Cast Steel. } \\
\hline $\mathscr{H}$ & $\mathscr{B}$ & $\mu$ & $\mathscr{H}$ & $\mathscr{B}$ & $\mu$ & $\mathscr{H}$ & $\mathscr{B}$ & $\mu$ \\
\hline 10 & I 2,400 & $\mathrm{I}, 240$ & Io & 5,000 & 500 & Io & 9,700 & 970 \\
\hline 20 & I 4,330 & 716 & 20 & 6,600 & 330 & 20 & 13,380 & 669 \\
\hline 30 & I 5,100 & 503 & 30 & 7,290 & 246 & 30 & I 4,500 & 483 \\
\hline 40 & 15,550 & 389 & 40 & 7,850 & I95 & 40 & 15,250 & 381 \\
\hline 50 & I5.950 & 319 & 50 & 9,360 & 169 & 50 & 15,840 & 317 \\
\hline 60 & I6,280 & $27 \mathrm{I}$ & 60 & 8,800 & 146 & 60 & 16,300 & 272 \\
\hline 70 & 16,500 & 235 & 70 & 9,200 & 131 & 70 & 16,750 & 239 \\
\hline
\end{tabular}

57. Magnetic permeability.-A long coil of wire produces a magnetic field of intensity $\mathscr{H}$ in its (air-filled) interior, and this field intensity is of course equal to the magnetic flux per unit

* Thompson, Knight, and Bacon, Transactions American Institute of Electrical Engineers, Vol. IX, 1892. 
sectional area (the flux density) in the air-filled interior of the coil. If the region inside of the coil is filled with iron, the flux density is many times as great as $\mathscr{H}$, as is evident from an inspection of the $\mathscr{B}$ and $\mathscr{H}$ curves of Fig. 65. From this fact there arises the idea of the multiplying effect of an iron core, in so far as the production of magnetic flux is concerned. In conformity with this idea we may write:

$$
\mathscr{B}=\mu \mathscr{H}
$$

This quantity, $\mu$, is called the permeability of the iron. It is of course not a constant for a given kind of iron (if it were constant the $\mathscr{B}$ and $\mathscr{H}$ curve would be a straight line), but its value varies with the flux density $\mathscr{B}$, as is clearly shown in the tabulated values of $\mathscr{H}, \mathscr{B}$, and $\mu$ in Art. 56 .

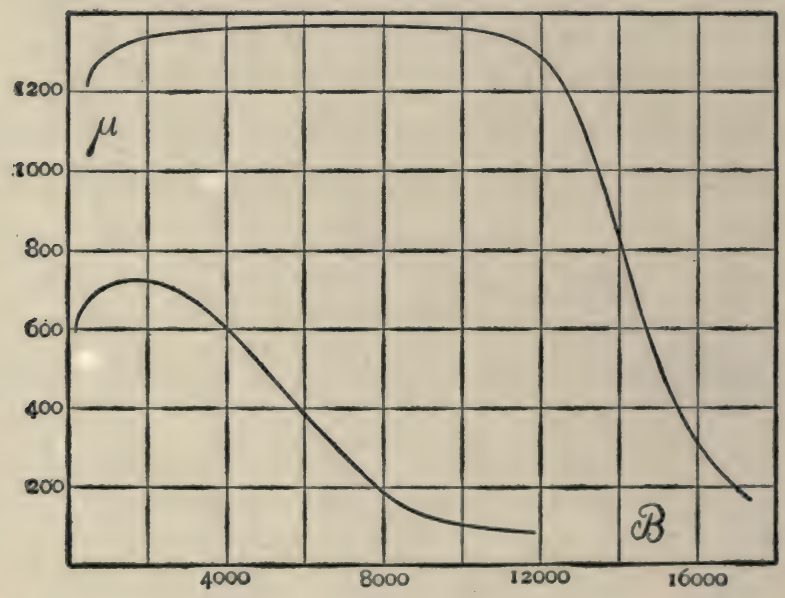

Fig. 66.

$\mu$ and $\mathscr{B}$ curves for wrought iron and cast iron.

The ordinates of the curves in Fig. 66 show the values of the permeability $\mu$ of wrought iron and of cast iron for various values of the flux densicy $\mathscr{B}$.

The permeability of air and of all non-magnetic materials such as copper, cotton, wood, etc., is unity. 
58. Magnetic circuit calculations. - When an iron rod of uniform size and quality forms a closed magnetic circuit and is subjected to a uniform magnetizing force $\mathscr{H}$ along its entire length, the value of the flux density $\mathscr{B}$ in the rod may be found from the $\mathscr{B}$ and $\mathscr{H}$ curve, and the total flux through the rod may be found by multiplying this flux density by the sectional area of the rod. Or, the uniform value of $\mathscr{H}$ required to give a specified total flux through the rod may be taken from the $\mathscr{B}$ and $\mathscr{H} t$ curve, after the value of $\mathscr{B}$ has been found, by dividing the specified flux by the sectional area of the rod.

When, however, the iron rod varies in size from point to point, or when different parts of the rod are of different kinds of iron, or when there is an air gap in the magnetic circuit, then some kind of an averaging process must be used to calculate the magnetomotive force required to produce a specified flux through the rod, or, conversely, to calculate the flux produced by a specified magnetomotive force.

This calculation is carried out as follows:

(a) To find the magnetomotive force required to produce a specified flux.-Divide the prescribed flux by the sectional area in square centimeters of each part of the circuit, wrought iron, cast iron, or air, as the case may be. This gives the flux density $\mathscr{B}$ in each part of the magnetic circuit.* Knowing $\mathscr{B}$ for each part of the circuit, take from the $\mathscr{B}$ and $\mathscr{H}$ tables or curves for the different kinds of iron, the value of $\mathscr{H}$ required to produce the known value of $\mathscr{B}$ in each part of the circuit, and multiply the value of $\mathscr{H}$ for each part of the circuit by the length in centimeters of that part. This gives the magnetomotive force required for each part; and the sum of these magnetomotive forces is the total magnetomotive force required. This total magnetomotive force may then be reduced to ampere-turns by multiplying it by $10 / 4 \pi$.

* It is here assumed that the whole of the magnetic flux passes through each portion of the iron circuit. This is not always the case, for a portion of the magnetic flux may stray through the air. This portion is called the leakage flux. Magnetic leakage is discussed in treatises on dynamo design. 
In this kind of a calculation it is to be remembered that flux density in air is $\mathscr{H}$, so that the flux density in the air gap is to be multiplied by the length in centimeters across the gap to give the magnetomotive force required for the gap.

(b) To find the flux produced by a specified magnetomotive force. - In the case of a rod of uniform size and quality this problem $(b)$ is simply the reverse of problem $(a)$. When, however, the parts of a magnetic circuit are of different sizes or of different materials, the flux produced by a specified magnetomotive force is best determined as follows: Calculate, as explained under $(a)$, the magnetomotive force required to produce a series of arbitrarily assumed values of flux. Arrange these results in tabular form, plot them as a curve, and from this plot find the flux corresponding to the prescribed magnetomotive force.

59. Analogy between the magnetic circuit and the electric circuit. Definition of magnetic reluctance.-The statements given in Art. $58(a)$ and $(b)$ are complete statements of the fundamental principles and methods of calculation of the magnetic circuit. A slight modification of the fundamental methods outlined in Art. 58 is, however, extensively used. This modified method is based upon an analogy between the magnetic circuit and the electric circuit, but it contains no physical or mathematical principles in addition to those involved in the fundamental method outlined in Art. 58 , and its only advantage is that the fundamental equation is rearranged so as to correspond exactly in form to the familiar equation for Ohm's law.

The fundamental equation

$$
\mathscr{B}=\mu \mathscr{H}
$$

as applied to an iron rod which forms a magnetic circuit may be transformed as follows: The magnetic flux $\Phi$ through the rod is equal to $\mathfrak{B}_{S}$, whence equation (I) may be written:

$$
\Phi=\mu \varsigma \mathscr{H}
$$


Now the magnetomotive force $\mathscr{F}$ along the rod is equal to $\mathscr{H}$ where $l$ is the length of the rod. Substituting, therefore, $\frac{\mathscr{F}}{l}$ for $\mathscr{H}$ in equation (2) we have:

$$
\Phi=\frac{\mu S \mathcal{F}}{l}
$$

This equation may be rewritten thus:

$$
\Phi=\frac{\mathscr{F}}{\mathscr{R}}
$$

in which $\mathscr{R}$ is written for $\frac{\mathrm{I}}{\mu} \cdot \frac{l}{s}$. That is,

$$
\mathscr{R}=\frac{\mathbf{I}}{\mu} \cdot \frac{l}{s}
$$

The quantity $\mathscr{R}$ is called the reluctance of the magnetic circuit, and the reciprocal of the permeability of the iron, $I / \mu$, is called its specific reluctance or reluctivity.

A portion of a magnetic circuit one centimeter in length $(l=\mathrm{I})$, and one square centimeter in sectional area $(s=\mathrm{I})$, made of a material having a permeability of unity $(\mu=\mathrm{I}$, which is the value of $\mu$ for air), has unit reluctance. The name oersted has been adopted for this unit of reluctance by the American Institute of Electrical Engineers.

Equation (4) is exactly similar in form to the equation expressing Ohm's law, namely, $I=E / R$; and equation (5) is similar in form to the equation for calculating the resistance of a wire, having given its length and section and the specific resistance or resistivity of its material. This analogy between the magnetic circuit and the electric circuit is, however, physically incomplete.

To find the magnetomotive force required to produce a specified magnetic flux, using equations (4) and (5), proceed as follows: Divide the total flux by the sectional area of each portion of the magnetic circuit thus finding the flux density for each portion. Knowing the flux density $\mathscr{B}$ for each portion of the circuit, 
take the corresponding values of $\mu$ from the table in Art. 56. Knowing the value of $\mu$, the length $l$, and the sectional area $s$ of each portion of the circuit, calculate the magnetic reluctance $\mathscr{R}$ of each portion. Add these separate reluctances together to get the total reluctance of the entire circuit, and multiply the total flux $\Phi$ by this total reluctance to get the required magnetomotive force $\mathscr{R}$, according to equation (4).

6o. Work required to magnetize iron.-When an iron rod is magnetized by sending an electric current through a coil of wire surrounding the rod, an opposing electromotive force is induced in the coil by the growing magnetism of the rod, and the work done in forcing the current against this opposing electromotive force is the work expended in magnetizing the rod.

The work $W$, in ergs, which is done in magnetizing $V$ cubic centimeters of iron from a given initial flux density $\mathfrak{B}^{\prime}$ to a given final flux density $\mathfrak{B}^{\prime \prime}$, is given by the equation:

$$
W=\frac{V}{4 \pi} \int_{\mathscr{B}^{\prime}}^{\mathscr{B}^{\prime \prime}} \mathscr{H} \cdot d \mathfrak{B}
$$

Proof.-In order to avoid the complications which arise on account of the perceptible demagnetizing action of the poles of a short iron rod, let us consider a very long slim rod $l$ centimeters in length and $s$ square centimeters in sectional area. Suppose this rod to be placed in a long coil having $z$ turns of wire per centimeter of length or $l z$ total turns. When the coil of wire is first connected to the battery or other source of current, the current in the coil (beginning at zero) rises in value during the time that the rod is being magnetized, and during this time the magnetic flux through the rod is growing in value. Let $d \Phi / d t$ be the rate at which the flux is increasing at a given instant, and let $i$ be the value of the current at this instant. Then $l z \times d \Phi / d t$ is the induced electromotive force in the coil which at the given instant is opposing the current $i$, so that $l z \times d \Phi / d t$ $X i$ is the rate, $d W / d t$, at which work is being done at the given instant in magnetizing the rod. That is: 


$$
\frac{d W}{d t}=l z i \cdot \frac{d \Phi}{d t}
$$

so that:

$$
d W=l z i \cdot d \Phi
$$

in which $d W$ is the amount of work done during the time that the flux has increased by the amount $d \Phi$ and while the current has the mean value $i$.

Now $\Phi=\mathscr{B} s$ or $d \Phi=s \cdot d \mathscr{B}$ from equation (I) of Art. 54, and $z i=\mathscr{H} / 4 \pi$ from equation (I) of Art. I 7 , so that the equation (2), above, becomes:

$$
d W=\frac{l s}{4 \pi} \mathscr{H} \cdot d \mathscr{B}
$$

or, since $l s=V$, we have:

or

$$
d W=\frac{V}{4 \pi} \mathscr{H} \cdot d \mathfrak{B}
$$

$$
W=\frac{V}{4 \pi} \int_{\Re^{\prime}}^{\Re \prime} \mathscr{H} \cdot d \mathfrak{B}
$$

In magnetizing a short iron rod, more work is done than is accounted for by equation (I) above. The additional work goes to establish the magnetic field in the neighborhood of the magnetic poles of the rod. Equation (I), above, expresses the work which is spent within the iron.

6r. Graphical representation of work done in magnetizing iron.-Let the curve $o p p^{\prime}$, Fig. 67 , be drawn so that the coordinates represent corresponding values of $\mathscr{B}$ and $\mathscr{H}$ for a given sample of iron. The branch op represents the values of $\mathscr{B}$ and $\mathscr{H}$ when the iron is being magnetized for the first time, and the branch $p p^{\prime}$ represents the values of $\mathscr{B}$ and $\mathscr{H}$ when, after the iron has been magnetized up to the point $p$, the value of $\mathscr{H}$ is slowly reduced to zero. The curve of $\mathscr{B}$ and $\mathscr{H}$ for decreasing values of $\mathscr{H}$ does not coincide with the curve for increasing values of $\mathscr{H}$. Now, as explained later, the total area opa represents the work done upon the iron in magnetizing it up to the point $p$, and the area $p p^{\prime} a$ represents the work which is regained from 
the iron when the magnetizing field drops slowly to zero. The work regained is less than the work required to magnetize the iron.

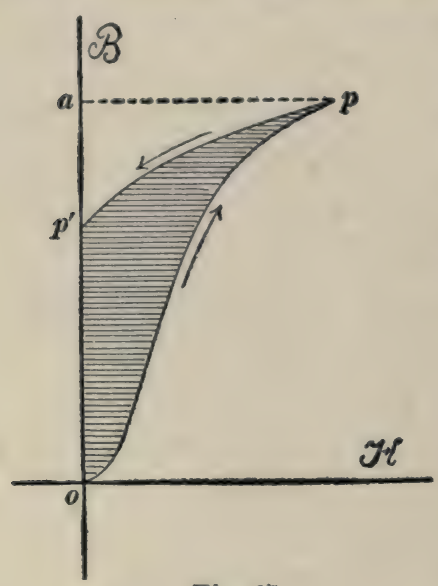

Fig. 67. The work which is lost is represented by the shaded area in Fig. 67.

That area in Fig. 67 represents work may be shown as follows: Abscissas represent values of $\mathscr{H}$ to scale, so that we may write:

$$
\mathscr{H}=a x
$$

Ordinates represent values of $\mathscr{B}$ to scale, so that we may write:

$$
\mathscr{B}=\text { by }
$$

or

$$
d \mathscr{B}=b \cdot d y
$$

Substituting these values of $\mathscr{H}$ and $d \mathscr{B}$ in equation (I) of Art. 60 we have

$$
W=\frac{a b V}{4 \pi} \int x \cdot d y
$$

in which $a$ is the number of units of $\mathscr{H}$ represented by one unit of abscissa, and $b$ is the number of units of $\mathscr{B}$ represented by one unit of ordinate in Fig. 67. Now $\int x \cdot d y$ is the area between any portion of the $\mathscr{B}$ and $\mathscr{H}$ curve and the $y$-axis. Therefore, $a b V / 4 \pi$ is the number of ergs of work represented by each unit of area between the $\mathscr{B}$ and $\mathscr{H}$ curve and the $y$-axis.

62. Magnetic hysteresis. The magnetic cycle.-The divergence of the $\mathscr{B}$ and $\mathscr{H}$ curve for increasing values of $\mathscr{H}$ from the $\mathscr{B}$ and $\mathscr{H}$ curve for decreasing values of $\mathscr{H}$ is called magnetic hysteresis; or, rather, the tendency of iron to retain a previous magnetic state which is the cause of this divergence is called magnetic hysteresis. One effect of magnetic hysteresis is that the work regained when iron is demagnetized is less than the work which must be spent to magnetize the iron, as pointed out in Art. 61 . 
The magnetization of a given portion of the iron core of a transformer is reversed with each reversal of the alternating current which flows through the primary coil, the iron is thus repeatedly carried from a certain degree of magnetization in one direction (a certain positive value of $\mathscr{B}$ ) to the same degree of magnetization in the opposite direction (the same negative value of $\mathfrak{B}$ ) and back to the original degree of magnetization. Such a magnetic double-reversal is called a magnetic cycle. At the end of a cycle the iron comes to precisely the same condition that it had at the beginning of the cycle. The importance of the magnetic cycle in the determination of the amount of heat produced by magnetization will be appreciated from the following two statements:

(a) When a mass of iron is magnetized along the $\mathscr{B}$ and $\mathscr{H}$ curve $o p$ of Fig. 67 and then partially demagnetized along the curve $p p^{\prime}$, a portion of the work done upon the iron during the first stage $o p$ is regained during the stage $p p^{\prime}$, a portion is lost as heat, and a portion remains in the iron as energy of magnetization; and no experimental method has been devised for determining the second and third portions of work separately.

(b) When, however, a mass of iron is carried through a magnetic cycle, the algebraic sum of the work spent upon the iron during the cycle is lost as heat, inasmuch as the magnetic energy in the iron is exactly the same

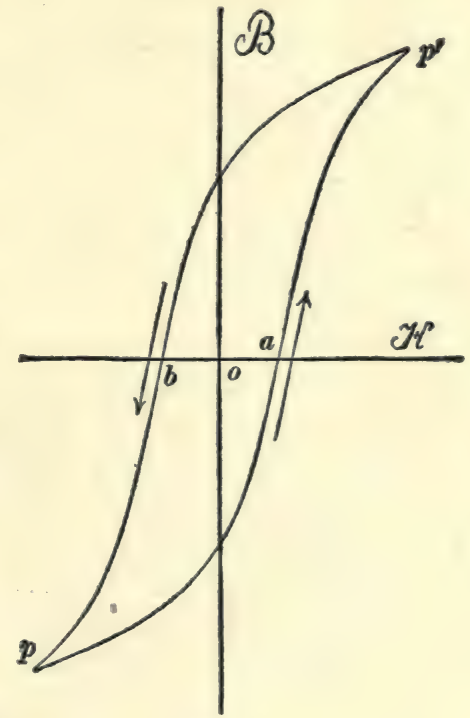

Fig. 68. at the beginning and at the end of the cycle.

Figure 68 shows the relation between $\mathscr{B}$ and $\mathscr{A}$ during a 
complete magnetic cycle. The total work spent on the iron is given by the value of the integral

$$
\frac{a b V}{4 \pi} \int x \cdot d y
$$

extended over the whole cycle; but the value of $\int x \cdot d y$ extended over the whole cycle is the area enclosed by the $\mathscr{B}$ and $\mathscr{H}$ curve. Therefore, the total energy, in ergs, lost in $V$ cubic centimeters of iron per magnetic cycle, is equal to $a b V / 4 \pi \times$ area enclosed by the $\mathscr{B}$ and $\mathscr{H}$ curve. This energy loss is called the hysteresis loss, and it is all converted into heat. The meanings of $a$ and $b$ are explained in Art. 6r.

The hysteresis loss per cycle increases with the range of flux density, and it may be expressed with sufficient accuracy for most practical purposes by the empirical equation:

$$
W=\eta V \mathfrak{B}^{1 \cdot 6}
$$

which is due to Steinmetz. In this equation $W$ is the loss of energy in ergs per cycle, $V$ is the volume of the iron in cubic centimeters, $\pm \mathscr{B}$ is the range of flux density during the cycle, and $\eta$ is a coefficient which is nearly constant for a given kind of iron or steel. The following table gives the approximate values of $\eta$ for different kinds of iron and steel.

TABLE.

Values of hysteretic coefficient $\eta$.

Best quality of sheet iron for transformer cores, annealed...... 0.0015

Sheet iron for armature cores, annealed . . . . . . . . . . . 0.003

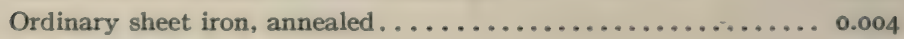

Soft annealed cast iron. . . . . . . . . . . . . . . . . . . . 008

Soft machine steel. . . . . . . . . . . . . . . . . . . . . . 0.0095

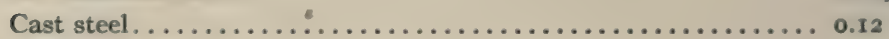

Hardened steel. . . . . . . . . . . . . . . . . . . . . . .

\section{PROBLEMS.}

I. The intensity of the magnetic field in the air gap between the pole face and the armature core of a dynamo is 3,500 gausses and the field is at right angles to pole face and armature surface. 
The distance across the air gap is $3 / 8$ inch. Find the magnetomotive force across the air gap in gilberts and in ampere-turns. Ans. 3,333.5 gilberts or 2,650 ampere-turns.

2. Find the magnetomotive force in gilberts and in ampereturns along a vertical line Io meters long at a place where the intensity of the earth's magnetic field is 0.56 gauss and its dip is $72^{\circ}$. Ans. 533 gilberts or 424 ampere-turns.

3. A slim rod 25 centimeters long is made into a link which passes through a coil of 50 turns of wire in which a current of I5 amperes is flowing. Find the average value along the rod of the component parallel to the rod of the magnetic field due to the coil. Express the result in gausses. Ans. 37.7 gausses.

4. An iron ring is wound with $I, 000$ turns of wire, and the wire has a current of 2 amperes flowing in it. The ring has an air gap in it and the distance across the gap space is 0.75 centimeter. Find the magnetic field intensity in the gap space assuming that the magnetomotive force of the winding is all concentrated on the gap space. Ans. 3,350 gausses.

5. An iron rod 2 centimeters square and 20 centimeters long is magnetized to an intensity of $\mathrm{I}, 000$ units pole per square centimeter section when it is placed in a region which, but for the action of the poles of the rod, would be a uniform field parallel to the rod and of an intensity of 102 gausses. Assuming the poles of the rod to be concentrated at its ends, calculate the net magnetizing field at the center of the rod. Ans. 22 gausses.

6. Find the total magnetic flux through the middle part of the rod specified in problem 5. Ans. 50,353.6 maxwells or lines.

Note.-One part of the flux is $4 \pi m$ and the other part is $\mathscr{H} \mathcal{S}$, where $\mathscr{H}$ is the net magnetizing field at the middle of the rod and $s$ is the sectional area of the rod.

7. A bar magnet of hard steel is 2 centimeters square and 20 centimeters long, and the strength of each pole of the magnet is 2,000 units. The magnet is placed in a region which, but for the presence of the magnet, would not be a magnetic field. 
Find the total magnetic flux through the middle part of the magnet. Ans. 24,972.8 maxwells.

Note.-In this case the net magnetizing force, $\mathscr{H}$, at the middle of the bar is a demagnetizing force, and the flux due to $\mathscr{H}$ is subtracted from $4 \pi m$.

8. A transformer has a sheet iron core of which the uniform sectional area is 120 square centimeters. The mean length of the magnetic circuit formed by the core is Ioo centimeters. How much current must be passed through a winding of 500 turns of wire to produce a magnetic flux of $1,767,000$ lines through the core? Ans. 3.88 amperes.

9. How much flux will be produced through the wrought iron core, specified in problem 8 , by a current of 8.2 amperes through the 500 turns of wire? Ans. I,920,000 lines.

I0. The magnetic circuit of a dynamo consists of wrought iron (in armature core and in field cores), cast iron (in field yoke and pole pieces), and air gap. The wrought iron portion is 50 centimeters long and I2O square centimeters in sectional area, the cast iron portion is 40 centimeters long and 220 square centimeters in sectional area, and the air portion is 2.5 centimeters long and 300 square centimeters in sectional area. How many ampere-turns are required to force $1,600,000$ lines of flux through this circuit, ignoring magnetic leakage? Ans. I2, I00 ampereturns.

Note.-On account of magnetic leakage the magnetic flux through field yoke, field cores and pole pieces is larger, sometimes very much larger, than the flux which flows across the air gaps and through the armature core. Therefore a slightly greater magnetomotive force is required to overcome the magnetic reluctance of field yoke, field cores and pole pieces than would be required if the magnetic leakage were zero.

Ir. How much magnetic flux would be forced through the magnetic circuit specified in problem Io by a magnetomotive force of 13,500 ampere-turns? Ans. 1,723,000 lines.

Note.-This problem is to be solved by calculating a series of values of ampereturns for various assigned values of flux. These results are to be plotted. The flux corresponding to 13,500 ampere-turns may then be taken from this curve. 
I2. (a) Find the work in ergs spent in magnetizing a wrought iron bar 3 inches square and 20 inches long from a neutral condition to $\mathscr{B}=16,000$ lines per square centimeter, using the tabular values of $\mathscr{B}$ and $\mathscr{H}$ given in Art. 56. (b) Find the work in ergs required to magnetize a cast-iron rod of the same size from a neutral condition to $\mathscr{B}=9,000$ lines per square centimeter. Ans. (a) $32.6 \times 10^{6}$ ergs. (b) $33.9 \times 10^{6}$ ergs.

Note.-Plot the $\mathscr{B}$ and $\mathscr{H}$ curves from the tabular values given in Art. 56. Divide the areas between the curves and the $\mathscr{B}$ axis into a number of narrow strips, and calculate the area of each strip. Add these areas to get the total area.

13. A transformer core contains 96 cubic inches of the best quality of transformer iron. The core is carried through I33 magnetic cycles per second between the limits $\mathscr{B}= \pm 3,500$ gausses, by means of an alternating current. Find the hysteresis loss in the core in watts. Ans. 14.7 watts. 


\section{CHAPTER V.}

SHIP'S MAGNETISM AND THE COMPENSATION OF THE COMPASS.*

63. The ship's compass.-The style of ship's compass which is now almost universally used is that which is due to Lord Kelvin. The card of this compass is shown in Fig. 69. The

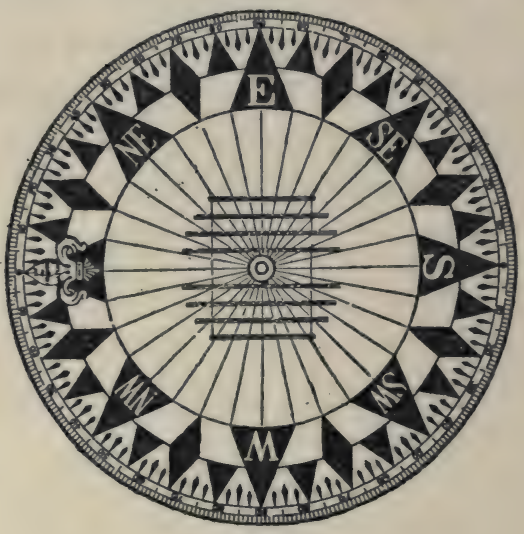

Fig. 69.

points of the compass and the circle divisions are printed on a paper ring to which is attached a light rim of aluminum which keeps it in shape. Radial threads connect the paper ring to a central disk which contains a sapphire cap by which the compass is supported on an iridium point. Eight small magnets of glass-hard steel are tied to the radial threads four on either side

* A good discussion of this subject is given in Gray's Treatise on Magnetism and Electricity, Vol. I, pages 85-100, Macmillan and Company, 1898. For full details, the reader is referred to Lord Kelvin's Instructions for Adjusting the Compass, to be obtained from James White, of Glasgow. The practice in the United States Navy concerning the matter of compass errors and compass adjustments is given in several small pamphlets which are published by the United States Navy Department, and in a book entitled $\boldsymbol{A}$ Treatise on Navigation, by Commander W. C. P. Muir, U. S. Navy, Annapolis, 1906. The practice in the British Navy is given in the Admiralty Manual of Deviations of the Compass. 
of the jewel cap, as shown in the figure. The entire weight of the card, including the magnetic needles, is about I70 grains, and this extreme lightness combined with the relatively large moment of inertia due to the distribution of the mass, insures a long period of free vibration and therefore great steadiness. The lightness of the card also gives a very small frictional resistance at the supporting point. The compass card with its attached needles is supported in a copper bowl which is supported on gimbals, so that the compass remains horizontal in spite of the rolling motion of the ship. The complete instrument is supported on a column which contains or supports the compensating devices which are explained later, and the entire arrangement is called the binnacle.

When a ship contains no iron or steel the compass points in the direction of the magnetic meridian.* When, however, the ship is made of iron or steel, or, when it carries a cargo of iron or steel, the compass is usually deflected by the magnetism of the ship or of its cargo. In order that a compass may be used for purposes of navigation under such conditions, the errors of the compass may be determined by a careful set of observations, or the influence of the ship's magnetism may be compensated so as to reduce the compass errors approximately to zero. The latter method is the one which is usually employed, and in some cases the residual errors which remain on account of incomplete compensation are determined by a careful set of observations and allowed for in the use of the compass.

64. Ship's magnetism. - A ball of iron which is devoid of permanent magnetism is weakly magnetized by the earth's field. This magnetism is not in a fixed direction in the ball, but is always in the direction of the earth's field however the ball may be held or turned. It is called the temporary magnetism of the ball, and it is proportional to the intensity of the earth's field. If the ball is elongated like an ellipsoid its temporary

* Magnetic north. 
magnetism is not in general parallel to the earth's field, and in the case of a long slim iron rod its temporary magnetism is always in the direction of its length and proportional to the component of the earth's field which is parallel to it, inasmuch as that component of the earth's field which is at right angles to a very slim rod produces no perceptible magnetism.

When a ball or rod of iron has a certain amount of permanent magnetism, the effect of the earth's magnetic field upon it is to produce an additional temporary magnetism, that is to say, the magnetism of the ball or rod is the sum of two distinct parts, a permanent magnetism and a temporary magnetism. Of course the permanent magnetism of a rod may be changed by severe mechanical shocks; the word permanent here refers to that part of the magnetism which does not change as the ball or rod is slowly moved around in the earth's field.

Similarly, an iron ship has a certain amount of permanent magnetism which does not change as the ship moves around in the earth's magnetic field, and a certain amount of temporary magnetism which is due to the magnetizing action of the earth's magnetic field.

65. Compass error due to permanent magnetism of a ship.The permanent magnetism of a ship produces at the compass

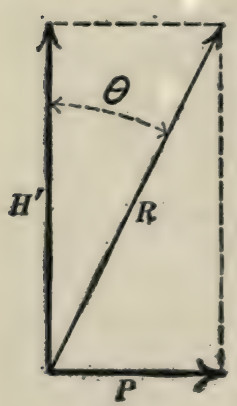

Fig. 70. box a magnetic field which is constant in value and fixed in direction with reference to the ship. The horizontal component of this field combines with the horizontal component of the earth's field to give a resultant field, and the compass needle points in the direction of this resultant. Thus, $H^{\prime}$ in Fig. 70 represents the horizontal component of the earth's field, $P$ represents the horizontal part of the magnetic field at the compass which is due to the permanent magnetism of the ship, $R$ represents the resultant horizontal field at the compass, and $\theta$ represents the compass error due to the ship's permanent magnetism. The 
field $P$ turns around with the ship, and therefore the compass error $\theta$ has a series of positive values (to the east) throughout a half revolution of the ship, and a series of negative values (to the west) throughout a half revolution of the ship. Therefore the compass error due to the ship's permanent magnetism is called the semicircular error.*

66. The semi-circular correctors.-The ideal compensation for the compass errors due to a ship's permanent magnetism would be to place a permanent steel magnet in such a position that it would produce, at the compass box, a magnetic field equal and opposite to the field produced at the compass box by the ship's permanent magnetism. As long as the ship remains on an even keel, however, it is only the horizontal part $P$, Fig. 70, of the field which is produced at the compass box by the permanent magnetism of the ship, which causes the deflection of the compass. Therefore it is sufficient to neutralize this horizontal field $P$ if the ship remains on an even keel. For this purpose, one or more horizontal magnets are placed in a tray in the pedestal of the binnacle and adjusted until they produce a field at the compass box which is equal and opposite to $P$. Usually, two such trays are employed, in one of which magnets are placed parallel to the line of the keel of the ship so as to annul the bow component of $P$, and in the other of which magnets are placed at right angles to the line of the keel so as to annul the athwartship component of $P$. These two trays with their permanent magnets are called the semicircular correctors.

\section{Compass errors due to temporary magnetism of a ship.-} An idea of the character of the compass errors which are due to the temporary magnetism of a ship may be obtained by imagining the ship to be a long slim bar $A B$, Fig. 7I, with a compass box at the point $C$. The earth's horizontal field $H^{\prime}$ may be resolved into two components, one parallel to $A B$ and

* The permanent magnetism of the ship contributes also to the heeling error which is discussed in Art. $7 \mathrm{I}$; and the so-called semicircular error is due partly to the temporary magnetism of the ship as explained in Art. 70. 
the other at right angles to $A B$. The component which is at right angles to $A B$ has no perceptible magnetizing action on $A B$, the component which is parallel to $A B$ causes the end $B$ to become a north pole and the end $A$ to become a south pole, and the magnetic field at $C$ due to these magnet poles is parallel

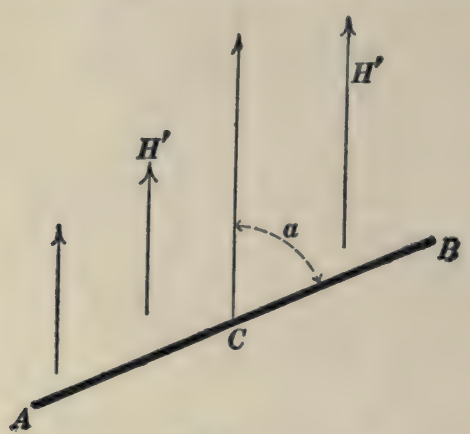

Fig. 71.

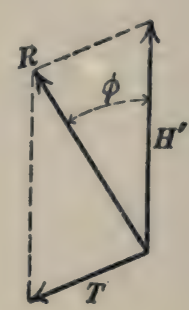

Fig. 72.

to $B A$ and towards $A$. The magnetic field at the compass box which is due to the temporary magnetism of the bar $A B$ in Fig. $7 \mathrm{I}$ is represented by the arrow $T$ in Fig. 72, the earth's field at the compass box is represented by $H^{\prime}$, and the arrow $R$ represents the resultant field at the compass box in the direction of which the compass needle points; therefore the angle $\phi$ is the compass error. Imagine $B$, Fig. $7 \mathrm{I}$, to represent the bow of the ship, and suppose the ship to start with its bow due north and swing around to the east so as to increase the angle $a$ from zero to $360^{\circ}$. A careful consideration of Figs. 71 and 72 will show that the angle $\phi$ has a series of westerly values throughout the first quadrant ( $a$ between zero and $90^{\circ}$ ), a series of easterly values throughout the second quadrant ( $a$ between $90^{\circ}$ and $180^{\circ}$ ), a second series of westerly values throughout the third quadrant ( $a$ between $180^{\circ}$ and $270^{\circ}$ ), and a second series of easterly values throughout the fourth quadrant. The compass error due to the temporary magnetism of a ship is therefore called the quadrantal error.

When the ship's compass is located on the center line of the 
ship so that the iron of the ship is symmetrically placed on the two sides of the compass, then the compass error due to the ship's temporary magnetism is zero when the ship heads north, east, south, or west, as may be shown as follows: When the ship heads magnetic north or south, its temporary magnetism is symmetrical as shown in Fig. 73; the magnetic field at the compass due to the
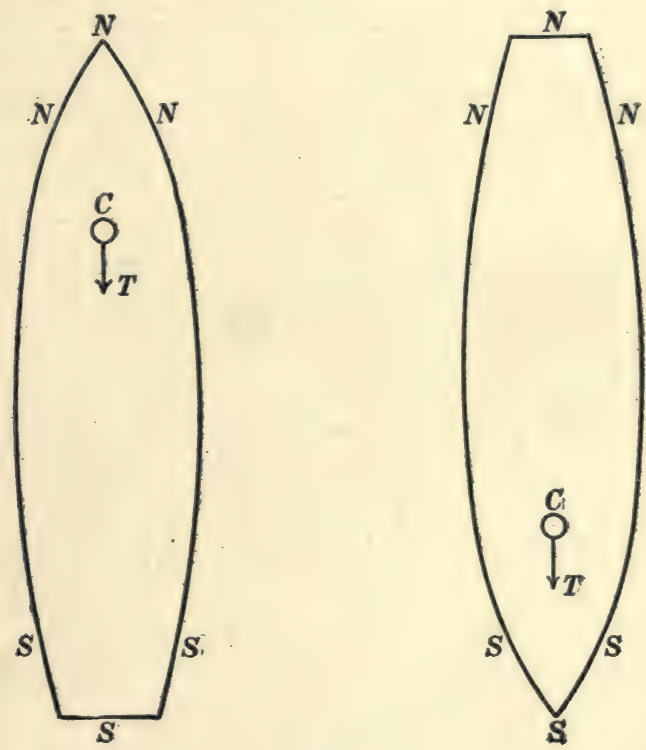

Fig. 73.

temporary magnetism of the ship is therefore due south, and consequently the compass is not deflected. Figure 74 shows a compass box $C$ placed on the center line of a ship of which the dissimilarity of bow and stern is greatly exaggerated. Imagine the vessel to be made of solid iron and consider the transverse slice of iron which lies between the dotted lines in Fig. 74 . When the bow points east or west (magnetic) the transverse slice is magnetized as indicated by the letters $N$ and $S$; and the curved line $f f$, which represents a line of force due to the poles $N$ and $S$, shows that the field, produced at $C$ by the magnetism of the transverse slice, is towards the south. What 
is here said concerning the given transverse slice of the ship is true of every transverse slice, and therefore the field at $C$ due
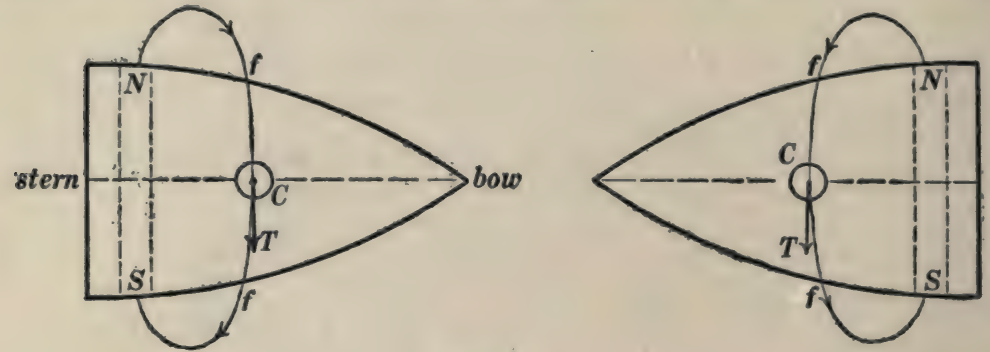

Fig. 74.

to the transverse magnetization of the entire ship when its bow points east or west (magnetic) is towards the south, and consequently the compass is not deflected.

68. Peculiarities of quadrantal error.-From Figs. 73 and 74 it is evident that the temporary magnetism of the ship weakens the field at the compass ( $T$ opposite to $H^{\prime}$ in direction, so that the resultant of $T$ and $H^{\prime}$ is less than $H^{\prime}$ ) when the ship heads

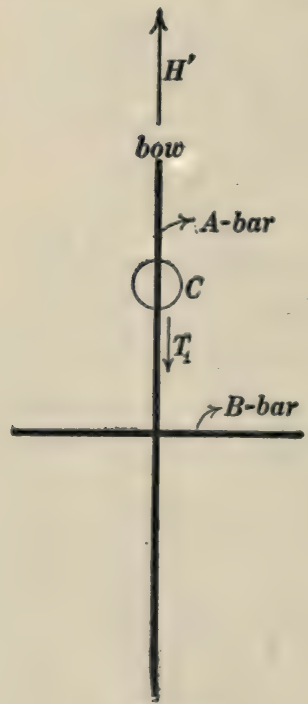

Fig. 75.

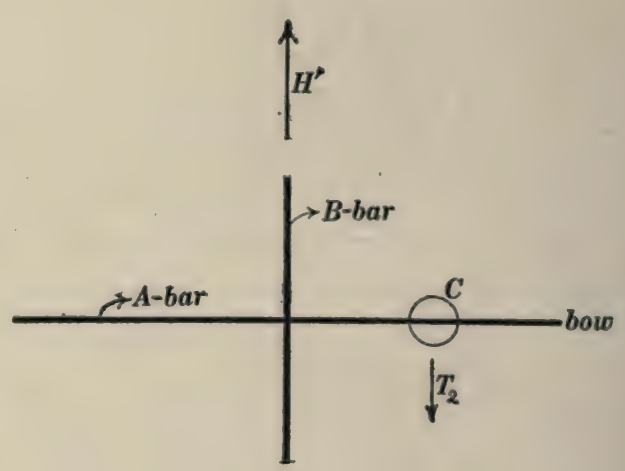

Fig. 76. 
north, east, south or west. If the value of $T$ is the same in Figs. 73 and 74, it can be shown that the temporary magnetism of the ship does not tend to deflect the compass, whatever the direction of the bow of the vessel.

To prove this proposition, we will assume that the iron of the ship is equivalent to two long slim horizontal bars of iron, one parallel to the ship's keel (the $A$-bar) and the other at right angles thereto (the $B$-bar). When the ship is headed north, as shown in Fig. 75, the full value of $H^{\prime}$ acts to magnetize the $A$-bar, and the magnetic field $T_{1}$, which is produced at the compass box by the magnetization of the $A$-bar, is proportional* to $H^{\prime}$ or equal to $k_{1} H^{\prime}$. When the ship is headed east, as shown in Fig. 76 , the full value of $H^{\prime}$ acts to magnetize the $B$-bar, and the magnetic field $T_{2}$, which is produced at the compass box by the magnetization of the $B$-bar, is proportional to $H^{\prime}$ or equal to $k_{2} H^{\prime}$. Therefore, if $T_{1}=T_{2}$, then $k_{1}=k_{2}$. The letter $k$ will be used in what follows for $k_{1}$ and $k_{2}$.

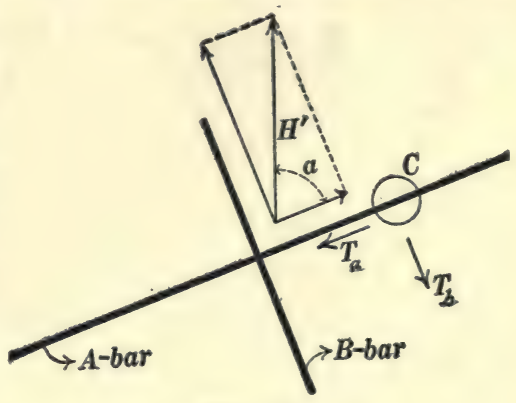

Fig. 77.

Consider the ship when it is headed $a$ degrees east of north as shown in Fig. 77. The component of $H^{\prime}$ which magnetizes the $A$-bar is $H^{\prime} \cos a$, and the magnetic field $T_{a}$ which is produced at $C$ by the magnetization of the $A$-bar is $k \times H^{\prime} \cos a$. The component of $H^{\prime}$ which magnetizes the $B$-bar is $H^{\prime} \sin a$, and the magnetic field $T_{b}$ which is produced at $C$ by the magnetization of the $B$-bar is $k \times H^{\prime} \sin a$. The resultant of $T_{a}$ and $T_{b}$ is

$$
\sqrt{T_{a}^{2}+T_{b}^{2}}=k H^{\prime} \sqrt{\cos ^{2} a+\sin ^{2} a}=k H^{\prime}
$$

Therefore the resultant of $T_{a}$ and $T_{b}$ is constant in value, and,

* Because the magnetization of the $A$-bar is proportional to $H^{\prime}$, and the field $T_{1}$ is proportional to the magnetization of the $A$-bar. 
since $T_{a}=k H^{\prime} \times \cos a$ and $T_{b}=k H^{\prime} \times \sin a$, it is evident that the resultant of $T_{a}$ and $T_{b}$ is always opposite to $H^{\prime}$ in direction, so that the actual field at the compass box is constant in value and always parallel and opposite to $H^{\prime}$, or, in other words, the compass error due to the temporary magnetism of the ship is zero on all headings of the ship when $T_{1}$ in Fig. 75 is equal to $T_{2}$ in Fig. 76.

69. Compensation of quadrantal error. The quadrantal correctors.-The quadrantal error of the ship's compass is eliminated

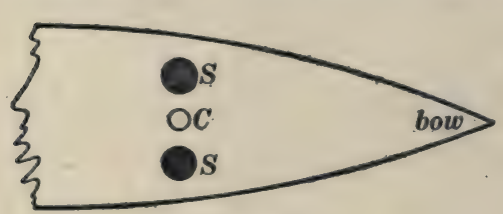

Fig. 78. (that is to say, compensated) by means of two iron spheres $S S$ which are usually* placed on the two sides of the compass, as shown to an exaggerated scale in Fig. 78. These spheres are called the quadrantal correctors, and the practical method of adjusting them is explained in Art. 73. The action of the quadrantal correctors may be understood with the help of Figs. $79 a$ and $79 b$ as follows: When the line joining the centers of the two spheres $S S$ is parallel to $H^{\prime}$ as shown in Fig. 79a, the magnetic field at the point $p$ is more intense than $H^{\prime}$; and when the line joining the centers of the spheres is at right angles to $H^{\prime}$ as shown in Fig. $79 b$, the magnetic field at $p$ is less intense than $H^{\prime}$. Now the weakening of the magnetic field at the compass box by the temporary magnetism of the ship is usually greater when the ship heads east or west than when the ship heads north or south. That is to say, $T$ is usually greater in Fig. 76 than it is in Fig. 75, or the coefficient $k_{2}$ is usually greater than the coefficient $k_{1}$ (see Art. 68). Therefore by placing the quadrantal correctors in the positions shown in Fig. 78 and moving them closer to, or farther away from the compass, the weakening of the magnetic field at the compass by the

* When the coefficient $k_{1}$ in Art. 68 is greater than $k_{2}$ the quadrantal correctors must be placed fore and aft of the compass box. This condition is, however, seldom realized in practice. 
combired temporary magnetism of ship and correctors may be made the same with the ship's head north (or south) as with

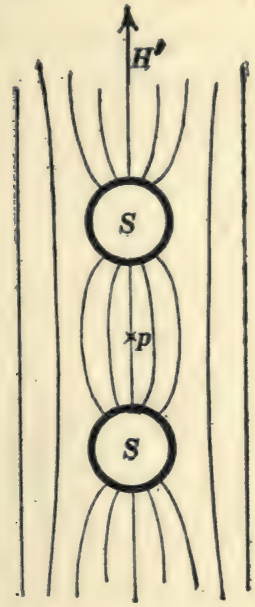

Fig. $79 a$.

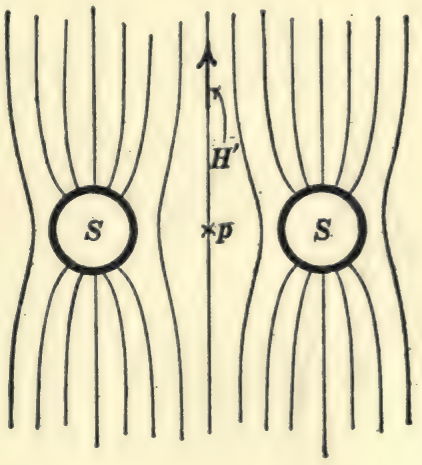

Fig. $79 b$.

ship's head east (or west), and when this condition is reached the quadrantal error of the compass is eliminated as explained in Art. 68.

70. Compass error due to the magnetizing action of the vertical component of the earth's magnetic field.-The vertical component $V$ of the earth's field produces a temporary magnetism of all the vertical iron in the ship; this "temporary" magnetism remains unaltered as long as $V$ remains unchanged, the ship being supposed to stand on even keel; and therefore the "temporary" magnetism due to $V$ merges with the permanent magnetism of the ship in the production of the semicircular compass error.

The temporary magnetism due to $V$ is distinguishable from the permanent magnetism of the ship, however, because it changes when the ship goes from one port to another where the value of $V$ is different. Thus, if the semicircular error is completely compensated at the home port by means of the semicircular 
correctors (permanent magnets), then a perceptible amount of semicircular error will appear when the ship sails to a distant port where the value of $V$ is different. In order to overcome this difficulty, that is, in order to compensate the semicircular error so that the compensation may hold good throughout a wide range of latitude, it is necessary to compensate, by means of the semicircular correctors, only that part of the semicircular

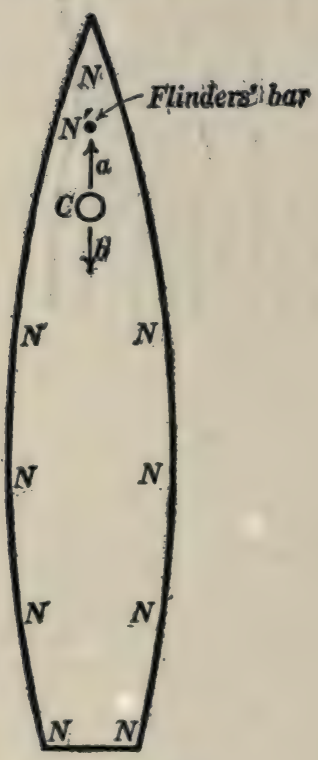

Fig. $80 a$.

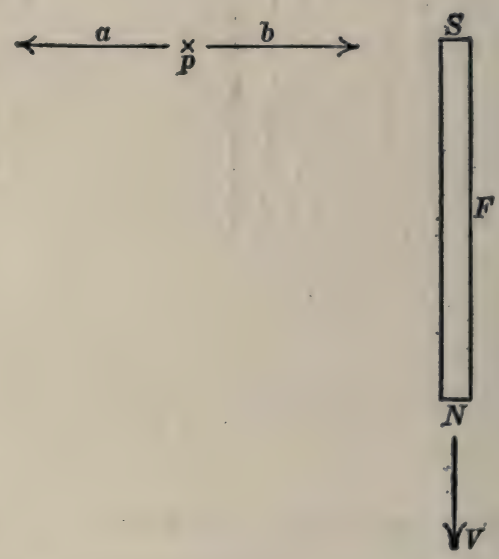

Fig. $80 b$.

error which is due to permanent magnetism, the remainder of the semicircular error (which is due to vertical temporary magnetism) being compensated by means of a vertical soft iron rod properly placed near the compass box. The use of this rod was proposed originally by Captain Flinders and it is usually called Flinders' bar. The action of Flinders' bar may be explained as follows: When $V$ changes in value, the magnetism of Flinders' bar and the vertical temporary magnetism of the ship change together. Flinders' bar being once for all adjusted to compensate the effect of the vertical temporary magnetism of the ship, the 
compensation holds whatever the value of $V$ may be. Flinders' bar is usually about three inches in diameter and from 6 to 24 inches long, according to the amount of iron in the vessel, and it is usually placed forward or aft of the binnacle.

Figure $80 a$ shows the north polarity $N N N N$, etc., on the deck of an iron vessel due to the vertical component of the earth's field. This north polarity is distributed symmetrically with respect to the ship's keel (ship's iron being symmetrical with respect to the ship's keel), and it produces, at the compass, a magnetic field of which the horizontal component is represented by the arrow $a$ which is parallel to the keel. Flinders' bar is placed in the position shown, and its north pole $N^{\prime}$ (upper end of bar), which is on a level with the compass box, produces at the compass box a field $b$ which is equal and opposite to $a$. Figure $80 b$ shows a side view of Flinders' bar $F$ (the compass box is supposed to be placed at the point $p$ ). Flinders' bar is magnetized by the vertical component $V$ of the earth's magnetic field, $a$ is the horizontal part of the field which is produced at the compass box by the vertical temporary magnetism of the ship's iron, and $b$ is the field produced at the compass box by Flinders' bar.

The method of adjusting Flinders' bar is explained in Art. 73.

7I. The heeling error.-Let us suppose that the semicircular and quadrantal errors have been completely compensated by means of the semicircular correctors and quadrantal correctors, the ship being all the time on an even keel. Under these conditions a deflection of the compass is produced when the ship rolls, or heels over, at sea. This deflection of the compass is called the heeling error, and it is due in part to the variation of the temporary magnetism of the ship which accompanies the change of direction of the earth's magnetic field with reference to the ship's iron as the ship rolls, and in part to the permanent magnetism of the ship. The dependance of heeling error upon the ship's permanent magnetism may be explained as follows: The horizontal field $P$, Fig. 7o, is annulled by the semicircular correctors, but the vertical component of the field produced at the compass box by the permanent magnetism of the ship is left unaltered by the semicircular correctors. By vertical component is here meant that component which is perpendicular to the ship's deck; as the ship rolls this component turns out of the true vertical and has a horizontal component at the compass, which deflects the compass. In the following discussion of the 
heeling corrector, the heeling error is assumed to be due entirely to the permanent magnetism of the ship.

Assuming the heeling error to be due entirely to the permanent magnetism of the ship, that is, to be due to the component $P^{\prime}$ (perpendicular to the deck). of the field which is produced at the compass box by the permanent magnetism of the ship, it is evident that the heeling error is a maximum when the ship heads north or south, and zero when the ship heads east or west; for, when the ship heels over with its head to the east or west, the part of $P^{\prime}$ which is projected upon a horizontal plane is directed towards the north or south and does not deflect the compass, whereas, when the ship heels over with its head north or south, the part of $P^{\prime}$ which comes into a horizontal plane is directed towards the east or west and it deflects the compass.

The heeling corrector is a vertical steel magnet placed directly beneath the compass box, and adjusted up or down until it produces at the compass box a vertical field which is equal and opposite to $P^{\prime}$. The practical method of adjusting the heeling corrector is explained in Art. 73.

72. Compass errors due to magnetic lag.-The temporary magnetism of the ship's iron tends to lag behind the magnetic field which produces it. Thus, after a ship has been standing for some time in one direction the magnetism which is produced by the earth's field does not at once disappear when the ship turns around, but tends to persist. This magnetic lag produces a compass error which is known as Gaussin's error. This error is never compensated in practice.

\section{Directions for adjusting the correctors of a ship's compass.} (a) Adjustment of semicircular correctors.-The quadrantal error is zero with ship's head north, east, south, or west. Therefore any deviation of the compass which exists on these headings is due to the semicircular error. With the ship's head north (magnetic), place one or more athwartship magnets in one of the semicircular-corrector trays and move them up or down until 
the compass points north. Then head the ship east (magnetic) and place fore and aft magnets in the other semicircular-corrector tray and move them up or down until the compass points north.

(b) Adjustment of quadrantal correctors. - Having corrected the semicircular deviation of the compass, head the vessel northeast (magnetic) or southeast, southwest, or northwest, and if any deviation of the compass exists, place the quadrantal spheres on the side brackets of the binnacle and move them in or out until the compass reading is correct.

(c) Adjustment of the heeling corrector.-With the ship headed north or south in a heavy sea, place the heeling-corrector magnet in its tube with its proper end upwards, and raise or lower it until the slow motion of the compass due to the rolling motion of the ship is nearly eliminated. The proper end up of the heeling-corrector magnet may be inferred as follows: Suppose that the north end of the compass needle is deflected to the east when the ship rolls to the west. Then it is evident that the perpendicular-to-the-deck component $P^{\prime}$ of the field-which-isproduced-at-the-compass-box-by-the-permanent-magnetism-ofthe-ship is downwards, because the part of it which is projected into a horizontal plane is to the east when the ship's masts roll to the west. In this case the north end of the heeling-corrector magnet is to be placed upwards so as to produce an upward field at the compass box.

(d) Adjustment of Flinders' bar.-Having carefully adjusted the semicircular correctors at the home port so as to annul completely the semicircular error, the ship is taken to a distant port and the semicircular error is observed with the ship's head east or west (magnetic). Let this error be represented by $\phi$; let $V$ and $H^{\prime}$ be the vertical and horizontal components of the earth's magnetic field at the home port and let $V_{1}$ and $H_{1}^{\prime}$ be the vertical and horizontal components of the earth's field at the distant port as determined by observation, or as taken from magnetic charts. The forward (or aft) component of the magnetic field which is produced at the compass box by the vertical 
temporary magnetism of the ship is proportional to the vertical component of the earth's field and it is therefore equal to $a V$ at the home port and equal to $a V_{1}$ at the distant port. The deviation of the compass (ship's head east or west) which is produced by this field is proportional to its intensity and inversely proportional to the horizontal intensity of the earth's field. Therefore this deviation is equal to $b V / H^{\prime}$ at the home port and equal to $b V_{1} / H_{1}^{\prime}$ at the distant port, where $a$ and $b$ are proportionality factors. Therefore the observed compass deviation $\phi$ is equal to $b\left(V / H^{\prime}-V_{1} / H_{1}^{\prime}\right)$, and the compass deviation $\phi_{t}$ which is due to the vertical temporary magnetism of the ship at the distant port is equal to $\frac{V_{1} / H_{1}{ }^{\prime}}{V / H^{\prime}-V_{1} / H_{1}{ }^{\prime}} \times \phi$. With the ship's head east at the distant port (the condition under which $\phi$ was observed), put Flinders' bar into a vertical position in front of, or behind the compass box, and move it towards or away from the compass until the compass is turned through an angle $\phi_{t}$ in a direction opposite to $\phi$, the angle $\phi_{t}$ being reckoned from the deflected position of the compass. Then eliminate the outstanding semicircular error by readjusting the semicircular correctors.

\section{PROBLEMS.}

I. The semicircular error of a compass on board ship is found to have a maximum value of $20^{\circ}$ to the east when the ship heads $36^{\circ}$ west of south. Make a sketch of the outline of the deck of the vessel and draw a line on the deck showing the direction of the horizontal component of the magnetic field at the compass box which is due to the permanent magnetism of the ship, find the value of this horizontal component and find the angle between its direction and the direction of the keel, the earth's horizontal field being equal to 0.2 gauss. Ans. (a) 0.068 gauss, (b) $106^{\circ}$ from bow towards port side (left side).

Note.-When the semicircular error is a maximum, the field $P$ at the compass due to the ship's permanent magnetism is at right angles to the resultant of $\boldsymbol{H}$ and $P$ as shown in Fig. $p r$. 
2. What is the value of the semicircular error of the compass when the ship specified in problem I heads $20^{\circ}$ north of east? Ans. $8^{\circ} 53^{\prime}$, west of north.

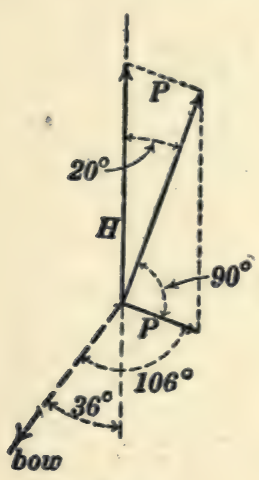

Fig. pr.

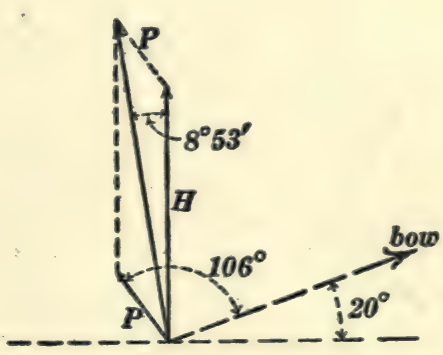

Fig. pa.

Note.-Figure $p_{2}$ shows the position of the boat's bow and the position of $P$.

3. The only error of a ship's compass is that which is due to the ship's permanent magnetism, the quadrantal error being compensated. The semicircular error has a value of $6^{\circ}$ to the west when the ship's head is true magnetic north and $4^{\circ}$ to the west when the ship's head is true magnetic northeast. On what true headings will the error of the compass be zero? Ans. 38 minutes south of east, and 38 minutes north of west.

Note. - The problem is to find the angle $\left(90^{\circ} 38^{\prime}\right)$ between the northerly direction and the line $P$ so that the lines $P$ and $Q$ (of the same length) may subtend angles of $6^{\circ}$ and $4^{\circ}$ respectively as seen from the point $O$.

4. Suppose that the semicircular error of the ship's compass has been completely compensated and suppose that the quadrantal error is ob-

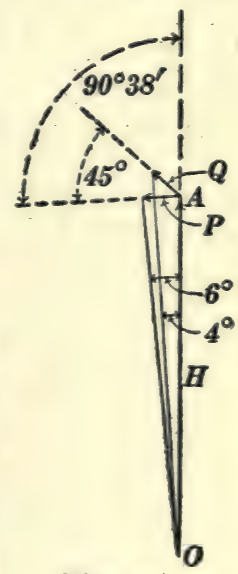

Fig. p3. served to be $4^{\circ}$ to the west when the ship is headed true northeast. What is the deviation of the compass when the ship heads $30^{\circ}$ south of east. Ans. $3^{\circ} 20^{\prime}$ to the east. 
Note.-In this problem treat the ship as one long slim iron bar parallel to the keel. The field $T^{\prime}$ is equal to $k H \sin \theta$ so that the value of the factor $k$ can be found from Fig. $p 4 a, \alpha$ being the known compass error and $H$ being taken as unity. The value of $T^{\prime \prime}$ is $k H \sin \phi$, so that the compass error $\beta$ can be calculated from Fig. $p 4 b$.

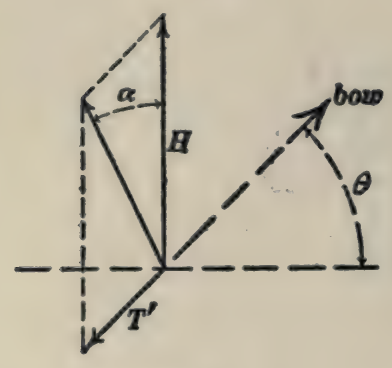

Fig. p4a.

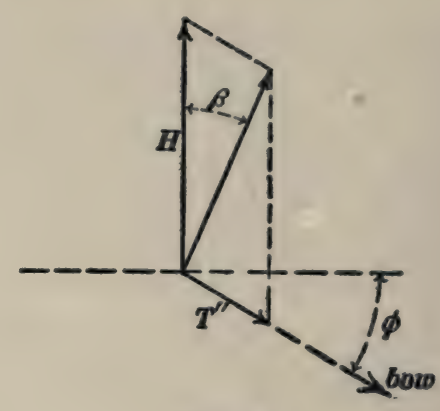

Fig. p4b.

5. A ship's head is due magnetic north and $P^{\prime}$ the perpendicular-to-the-deck component of the field at the compass due to the ship's permanent magnetism is downwards and equal to 0.05 gauss. Find the deflection of the compass due to heeling when the ship heels over $20^{\circ}$ to the west, the horizontal component of the earth's field at the compass being 0.I 6 gauss. Ans. $6^{\circ} 6^{\prime}$ to the east.

6. Find the deflection of the compass due to heeling when the ship heads magnetic northeast and heels $20^{\circ}$ to the southeast, other data being as in the previous problem. Ans. $4^{\circ}$ to the west. 


\section{CHAPTER VI.* \\ ELECTRIC CHARGE AND THE CONDENSER.}

74. Electric charge.-A current of water through a pipe is a transfer of water along the pipe. Let $q$ "be the amount of water which, during $t$ seconds, flows past a given point in a pipe, then the quotient $q / t$ is the rate of flow of water through the pipe, and this rate of flow may be spoken of as the "strength" $i$ of the water current. Therefore, if $i$ is the strength of water current (units of volume per second), then the amount of water flowing past a given point of the pipe in $t$ seconds is given by the equation

$$
q=i t
$$

Similarly, an electric current in a wire may be looked upon as a transfer of electricity along the wire, and the quantity $q$ of electricity which flows past a point on the wire during $t$ seconds may be defined as the product of the strength of the current and the time. That is:

$$
q=i t
$$

If the strength of the current is variable, then equation (I) must be written in the form:

$$
\Delta q=i \cdot \Delta t
$$

in which $\Delta q$ is the amount of charge which flows past a given point on the wire during a short interval of time $\Delta t$.

Volume of water is the fundamental and easily measured thing in hydraulics, and water current is most conveniently defined as cubic feet of water per second. In the case of electricity, however, the fundamental and easily measured thing is electric

* The usual elementary treatment of electrostatics is not sufficient as an introduction to Chapters VII and VIII. Therefore a very brief outline is given in this chapter. The student is urged to read pages I25-I 7I of Franklin and MacNutt's Elementary Electricity and Magnetism. 
current, and quantity of electricity is most conveniently defined as the product of strength of electric current and time.

Units of electric charge.-Quantity of electricity is usually spoken of as electric charge or simply as charge. The amperesecond is the amount of charge which flows in one second through a wire which carries a current of one ampere. The ampere-second is usually called the coulomb. One ampere-hour is the amount of charge flowing in one hour through a wire carrying one ampere. The ampere-hour is extensively used among electrical engineers in specifying the discharge capacity of storage batteries. The abcoulomb is the amount of charge which flows in one second through a wire carrying a current of one ab-ampere. One abcoulomb is equal to ten coulombs.

75. The momentary flow of current in an open circuit. Electrically charged bodies.-Figure $8 \mathrm{I}$ shows a battery maintaining

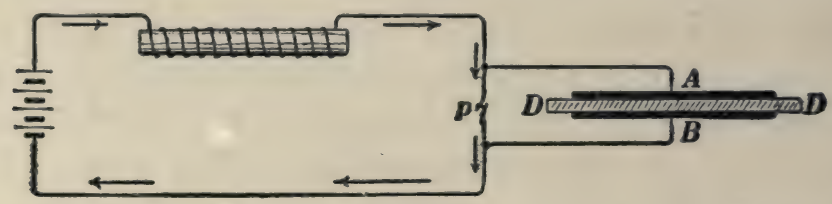

Fig. 81 .

an electric current through a circuit which contains a coil of wire on an iron core, and the circuit can be broken at will at the point $p$. Two metal plates $A$ and $B$ with a thin layer of insulating material $D D$ between them are connected as shown in the figure. When the plates $A B$ are not connected, a spark is produced when the circuit is broken at $p$, showing that the current continues to flow for a short time after the metallic contact at $p$ is broken. When the plates $A$ and $B$ are connected, however, there is no spark at $p$ when the circuit is suddenly broken. In this case the current does not continue to flow across the break at $p$; it flows into plate $A$ and out of plate $B$, and the two plates $A$ and $B$ are said to become electrically charged. The plate into which the momentary current flows is 
said to become positively charged, and the plate out of which the momentary current flows is said to become negatively charged.

The two metal plates $A$ and $B$ together with the layer of insulating material between them constitute what is called a condenser. A condenser is usually made of sheets of tin foil separated by sheets of waxed paper. Thus the heavy horizontal lines in Fig. 82 represent sheets of tin foil, and the finely dotted regions represent insulating material.

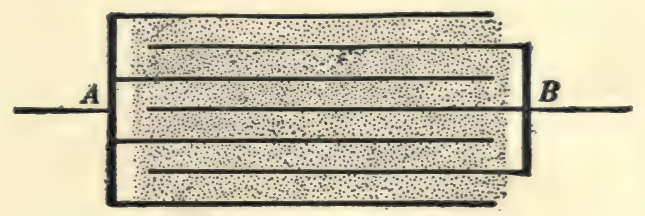

Fig. 82.

The flow of current in an open circuit may be shown by connecting a small incandescent lamp and a condenser in series to alternating-current supply mains. With each reversal of the alternating supply voltage a momentary current flows through the lamp, and the repeated pulses of current heat the lamp filament to incandescence.

The dielectric.-The insulating material between the metal plates $A$ and $B$ in Fig. $8 \mathrm{I}$ is called the dielectric. In general the insulating material surrounding a charged body or between two charged bodies is called a dielectric.

Electrostatic attraction.- The charged metal plates $A$ and $B$ in Fig. 8I have an evident attraction for each other when the dielectric is a fluid like air or oil. This electrostatic attraction of two charged metal bodies may be shown as follows: A gold leaf is hung along side of a vertical brass strip. When the gold leaf is connected to one terminal of a battery and the brass strip to the other terminal of the battery the gold leaf is attracted by the brass. It is necessary in this arrangement to cover the face of the brass plate with a layer of paper to prevent short circuiting the battery by the gold leaf. An electromotive 
force of 100 volts is sufficient to produce a very perceptible attraction with this arrangement.

76. Measurement of electric charge. The ballistic galvanometer.-A very large amount of electric charge may be determined by observing the time during which the charge will maintain a sensibly constant measured current. Thus the discharge capacity of a storage cell may be measured by observing the time during which the cell will deliver a current of, say, Io amperes. The amounts of charge which are most frequently encountered in the momentary flow of electric current in open circuits are, however, exceedingly small; and it is evident that a very small amount of electric charge cannot be measured by the method above suggested. Such small electric charges are measured by means of the ballistic galvanometer. This is a galvanometer usually of the D'Arsonval type with a fairly heavy moving coil. When a momentary pulse of current flows through such a galvanometer, the coil is set in motion, and a certain maximum deflection or throw of the coil is produced. Let $d$ be the measure of this maximum deflection or throw on the galvanometer scale. Then we have:

$$
q=k d
$$

in which $q$ is the amount of electric charge carried through the galvanometer coils by the momentary pulse of current, and $k$ is a constant for the given galvanometer. The value of the factor $k$ is generally determined in practice by sending through the galvanometer a known amount of charge and observing the throw $d$ produced thereby.*

77. The capacity of a condenser.-A ballistic galvanometer $B G$, a condenser and a number of dry cells are connected as shown in Fig. 83. One terminal of the condenser is connected to a flexible wire which is fixed to the end of a glass handle. By touching the wire $W$ to the point $b$, the electromotive force $E$

* The use of a ballistic galvanometer is discussed at some length in Absolute Measurements in Electricity and Magnetism by Andrew Gray, Vol. 2, pages 391-397. 
of one dry cell acts upon the condenser, and the momentary flow of "current which charges the condenser produces a throw of the ballistic galvanometer. The condenser can then be discharged by touching the wire $W$ to the point $a$. By touching the wire $W$ to the point $c$, the electromotive force $2 E$ of two dry cells

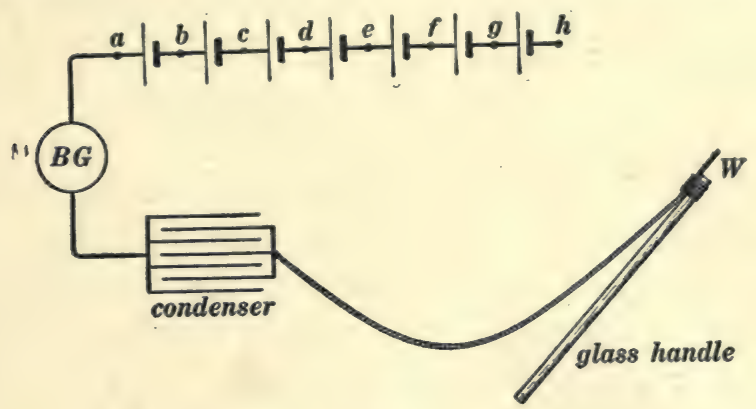

Fig. 83.

acts upon the condenser, and the momentary flow of current which charges the condenser produces a throw of the ballistic galvanometer. The condenser may then be discharged as before. By touching the wire $W$ to the point $d$, the electromotive force ${ }_{3} E$ of three dry cells acts upon the condenser, and the momentary flow of current which charges the condenser causes a throw of the ballistic galvanometer; and so on. In this way the throws of the ballistic galvanometer may be observed when the condenser is charged by an increasing series of voltages, $E, 2 E, 3 E$, $4 E$, and so forth, and it is found that the throw of the ballistic galvanometer becomes larger and larger in proportion to the voltage. But the throw of the ballistic galvanometer is proportional to the charge which is drawn out of one plate and forced into the other plate of the condenser. Therefore the amount of charge which is drawn out of one plate and forced into the other plate of a condenser is proportional to the electromotive force which acts upon the condenser. Therefore we may write:

$$
Q=C E
$$

where $Q$ is the quantity of charge which is drawn out of one 
plate and forced into the other plate of a condenser when an electromotive force of $E$ volts is connected so as to act upon the condenser, and $C$ is a constant for a given condenser. The factor $C$ is adopted as a measure of what is called the capacity of the condenser. Therefore, a condenser would have unit capacity if an electromotive force of one volt would draw one coulomb of charge out of one plate and force one coulomb of charge into the other plate of the condenser.

It is evident from the above equation that $C$, the capacity of a condenser, is expressed in coulombs-per-volt. One coulombper-volt is called a farad, that is to say, a condenser has a capacity of one farad when an electromotive force of one volt will draw one coulomb out of one plate of the condenser and force one coulomb into the other plate of the condenser.

Condenser capacities as usually encountered in practice are very small fractions of a farad. Thus the capacity of a condenser made by coating with tin foil the inside and outside of an ordinary one-gallon glass jar would be about one five-hundredmillionth of a farad, or 0.002 of a microfarad. A microfarad is a millionth of a farad, and in practice capacities of condensers are usually expressed in microfarads.

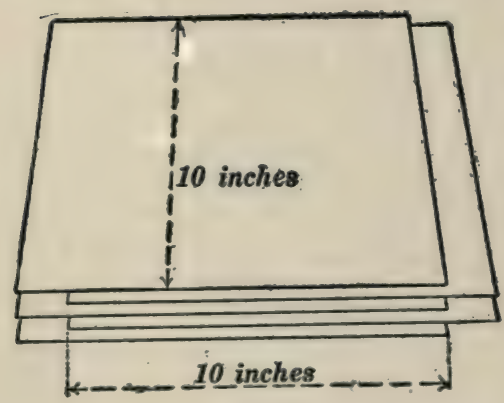

Fig. 84.

The approximate dimensions of a one-microfarad condenser are as follows: 5 or sheets of tin foil separated by sheets of paraffined paper 0.02 inch in thickness, the overlapping portions of the sheets of tin foil being Io inches by ro inches, as shown in Fig. 84 .

Two pieces of metal of any shape separated by insulating material constitute a condenser; the only reason for using sheets of metal with thin layers of insulating material between is to obtain a large capacity in a small space. 
78. Inductivity of a dielectric.-The capacity of a condenser depends upon the size of the plates, upon the thickness of the dielectric and upon the nature of the dielectric. The dependence of the capacity of the condenser upon the nature of the dielectric is a matter which must be determined purely by experiment. Thus Fig. 85 represents two metal plates with air between them, and Fig. 86 represents the same plates immersed in oil. The distance between the plates is understood to be the same in Figs. 85 and 86. Let $C$ be the capacity of the condenser in Fig. 85 with air as the dielectric, and let $C^{\prime}$ be the capacity of the condenser in Fig. 86 with a given kind of oil as the dielectric. The

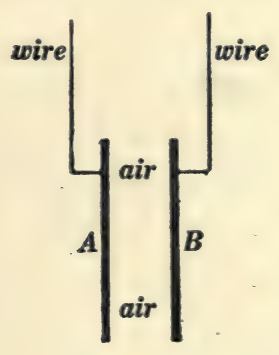

Fig. 85 .

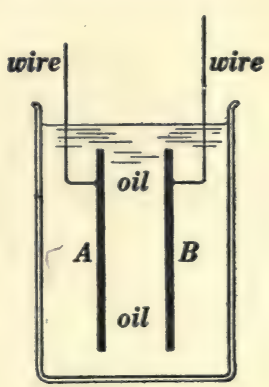

Fig. 86.

ratio $C^{\prime} / C$ is called the inductivity* of the oil. Thus the inductivity of kerosene is about 2.04, that is, the capacity of a given

TABLE.

Inductivities of Various Substances.

Crown glass (according to composition). $\ldots \ldots \ldots .3 .2$ to 6.9

Flint glass (according to composition). . . 6.6 to 9.9

Hard rubber...................... 2.08 to 3.01

Sulphur (amorphous)..................... 3.04 to 3.84

Paraffin................................. to 2.32

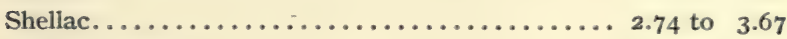

Ordinary $\operatorname{rosin} \ldots \ldots \ldots \ldots \ldots \ldots \ldots \ldots \ldots \ldots \ldots \ldots \ldots \ldots \ldots \ldots+2.48$ to 3.67

Mica (according to composition)........ 5.66 to Io

Petroleum................................ 2.04

Water....................... about 90 .

* What is here called the inductivity of a dielectric is sometimes called dielectric constant, or specific capacity of a dielectric, or specific inductive capacity of a dielectric. 
condenser is 2.04 times as great with kerosene between the plates as with air between the plates. The accompanying table gives the inductivities of a few dielectrics.

\section{Dependence of capacity of a condenser upon size and} distance apart of plates.-When the dielectric of a condenser is of uniform thickness and when the metal plates are large as compared with their distance apart (thickness of dielectric), then the capacity $C$ of the condenser is proportional* to $a / x$ for a given dielectric, where $x$ is the thickness of the dielectric and $a$ is the area of the sheet of dielectric between the plates. Therefore, if we choose a given dielectric, we may write

$$
C=G \cdot \frac{a}{x}
$$

in which $G$ is a constant. When $x$ is expressed in centimeters, and $a$ in square centimeters; when air is chosen as the dielectric; and when $C$ is expressed in farads; then the value of $G$ as found by experiment is $884 \times 10^{-16}$. Therefore we have:

$$
C_{\text {In farads }}=884 \times \mathrm{IO}^{-16} \times \frac{a}{x}
$$

When a dielectric whose inductivity is $k$ is used instead of air, the capacity of the condenser is $k$ times as great, or:

$$
C_{\text {In farade }}=884 \times \mathrm{IO}^{-16} \times \frac{k a}{x}
$$

in which $C$ is the capacity in farads of a condenser of which the plates are separated by a layer of dielectric $x$ centimeters thick and $a$ square centimeters in area (between the plates), and $k$ is the inductivity of the dielectric. The meaning of $a$ may be understood with the help of Fig. 84. If there are 50I sheets of tin foil there will be 500 sheets of dielectric, and $a$ will

* It can be shown from almost purely geometrical considerations that $C$ is proportional to $a / x$, but it is sufficient to accept this proportional relation as the result of experiment. The value of the proportionality factor $G$ must be determined by experiment directly or indirectly. 
be equal to $500 \times$ 10 inches $\times$ 10 inches or 322,500 square centimeters.

80. The work done by an electromotive force $E$ in pushing a given amount of charge, $Q$, through a circuit.-When $Q$ coulombs of electric charge flows through a battery of which the electromotive force is $E$, the amount of work $W$ done by the battery is $E Q$ joules. That is:

$$
W=E Q
$$

This is evident from the following considerations. Imagine a current $I$ flowing through the battery; then $E I$ watts is the rate at which the battery does work, and EIt joules is the amount of work done in $t$ seconds. But the product $I t$ is the amount of charge $Q$ (in coulombs) which has been pushed through the circuit. Therefore the work done, namely EIt joules, is expressible as $E Q$ joules. Therefore we have equation (I).

81. The potential energy of a charged condenser.-A charged condenser represents a store of potential energy in much the same way that a stretched spring represents a store of potential energy, and before considering the amount of potential energy in a charged condenser it is helpful to consider the amount of potential energy in a stretched spring.

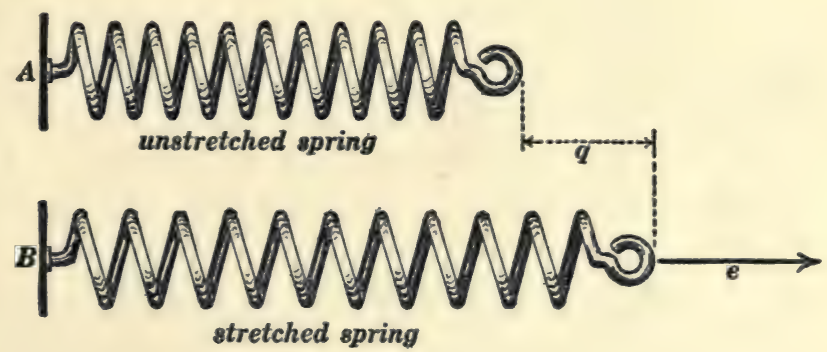

Fig. 87 .

Let $q$ represent the elongation of a spring due to a stretching force $e$ as shown in Fig. 87. As is well known $q$ is proportional to $e$; therefore if we plot corresponding values of $q$ and $e$ as 
abscissas and ordinates respectively, we will get a straight line cc as shown in Fig. 88.

Consider the total amount of work $W$ which is done while the spring is being stretched from $q=0$ to $q=Q$, and while the stretching force is increasing from $e=0$ to $e=E$. The average value of the stretching force is $\frac{1}{2} E$, as may be understood from Fig. 88, and the work done is equal to the product of the total stretch $Q$ and the average stretching force $\frac{1}{2} E$. That is:

$$
W=\frac{1}{2} E Q
$$

and this work $W$ is stored in the stretched spring as potential energy. Thus a stretch of 3 feet $(=Q)$ is produced in a large

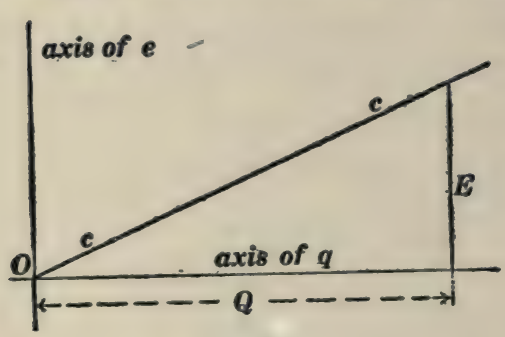

Fig. 88 . spring, and the stretching force rises from zero to 60 pounds $(=E)$. The average value of the stretching force is 30 pounds $\left(=\frac{1}{2} E\right)$, the work done is 90 foot-pounds ( $\left.=\frac{1}{2} E Q\right)$; and this work is stored in the stretched spring as potential energy.

Suppose a condenser to be charged by applying it to an electromotive force which begins at zero and rises to $E$ volts, then the amount of work $W$ which is done in charging the condenser is equal to $\frac{1}{2} E Q$ where $\frac{1}{2} E$ is the average value of the charging electromotive force, and $Q$ is the total charge which is drawn out of one plate of the condenser and pushed into the other plate. This statement is in accordance with equation (I) of Art. 80. Therefore:

$$
W=\frac{1}{2} E Q
$$

where $W$ is the potential energy of a charged condenser, $E$ is the voltage acting on the charged condenser, and $Q$ is the charge which has been drawn out of one plate of the condenser and pushed into the other plate; $W$ is expressed in joules when $E$ is in volts and $Q$, in coulombs. 
We may substitute $C E$ for $Q$ in equation (I), according to equation (I) of Art. 77, and we get:

$$
W=\frac{1}{2} C E^{2}
$$

or we may substitute $Q / C$ for $E$ in equation (I), according to equation (I) of Art. 77, and we get:

$$
W=\frac{1}{2} \frac{Q^{2}}{C}
$$

Following is a rigorous derivation of equation (3) as applied to a stretched spring. Let $\dot{q}$ be the elongation of the spring when the stretching force is $e$. Then $q$ and $e$ are proportional, so that:

$$
q=C e
$$

where $C$ is a constant for the given spring. Let $\Delta q$ be the added elongation due to an increment $\Delta e$ of the stretching force, and let $\Delta W$ be the work done on the spring to produce the added elongation. Then:

and

$$
\Delta W \text { is greater than } e \cdot \Delta q
$$

or

$$
\Delta W \text { is less than }(e+\Delta e) \cdot \Delta q
$$

$$
\frac{\Delta W}{\Delta q} \text { is greater than } e \text { and less than }(e+\Delta e)
$$

Therefore $\Delta W / \Delta q$ approaches $e$ as a limit when $\Delta e$ and $\Delta q$ both approach zero; or, using differential notation, we have $d W / d q=e$; or, using the value of $e$ from equation (4), we have:

$$
\frac{d W}{d q}=\frac{q}{C}
$$

Now the potential energy $W$ of the spring when its elongation is $Q$, is the amount of work done in stretching the spring from $q=0$ to $q=Q$, and this is found by integrating equation (5) from $q=0$ to $g=Q$, which gives:

$$
W=\frac{\mathrm{I} Q^{2}}{2 C}
$$


82. Disruptive discharge. Dielectric strength.-When the electromotive force which charges a condenser is increased more and more, the dielectric of the condenser is eventually broken down; this break down occurs in the form of an electric spark, it discharges the condenser, and it is called a disruptive discharge. By a condenser is here meant two metal bodies of any shape separated by insulating material. The electromotive force required to break down a dielectric depends upon three things, namely, $(a)$ the shape of the metal bodies, $(b)$ the minimum distance* between the metal bodies, and $(c)$ the nature of the dielectric. The dependence upon the shape of the metal bodies is illustrated by the fact that a given electromotive force will produce a much longer spark between points than between flat metal surfaces. In the following discussion the dielectric is assumed to be between flat metal plates.

When the dielectric is perfectly homogeneous like air or oil, the voltage required to break it down is very nearly proportional to its thickness, and the voltage required to break down such a dielectric divided by the thickness of the dielectric is called its specific dielectric strength. Thus the specific dielectric strength of air is about 35,000 volts per centimeter. When the dielectric is non-homogeneous the voltage required to break it down is not even approximately proportional to its thickness. The most familiar example of a non-homogeneous dielectric is the material which is used for insulating the windings of dynamos and transformers. This material is made up of layers of cloth and varnish and mica with occasional layers of air.

If a tank is made with one wall of porous material like unglazed earthenware, the pressure of the fluid in the tank has three important effects upon the wall, namely, $(a)$ a certain amount of fluid soaks through the wall, $(b)$ the wall is slightly elastic and it yields a little to the fluid pressure, and $(c)$ the wall has a certain ultimate strength and it will burst if the pressure exceeds a

* This is not true when the distance is very small or when the bodies are in a very good vacuum. 
certain amount. Similarly the electromotive force which acts on a condenser has three important effects upon the dielectric of the condenser, namely, $(a)$ a certain amount of electric current "soaks" through the dielectric, as it were, because the dielectric is an electrical conductor although a very poor one, (b) the dielectric has a certain amount of electrical "elasticity" (inductivity as it is properly called), and it "yields" a little to the electromotive force and allows a certain amount of charge to be drawn out of one plate and forced into the other plate of the condenser, and $(c)$ the dielectric has a certain ultimate strength and it will be ruptured if the electromotive force exceeds a certain amount.

\section{PROBLEMS.}

I. A variable current which is represented by $i=a t^{2}$ flows through a circuit. Find the charge which passes through the circuit between $t=0$ and $t=10$ seconds; the value of $a$ being such that the current will have reached a value of 18 amperes when $t$ is 3 seconds. Ans. 667 coulombs.

2. A variable current is represented by $i=I \sin \omega t$. (a) Find the amount of charge which passes through the circuit from $\omega t=0$ to $\omega t=\pi$. (b) Find the average value of the current during the time $\omega t=0$ to $\omega t=\pi$. Ans. (a) $2 I / \omega$ coulombs, (b) $2 I / \pi$ amperes.

3. Two condensers of which the capacities are $C$ farads and $c$ farads respectively are connected in parallel as shown in Fig. $p 3$. What is their combined capacity?

Note.-An electromotive force $E$ draws $c E$ coulombs out of one plate and forces it into the other plate of $c$; and the same electromotive force draws $C E$ coulombs out of one plate and forces it into the other plate of $C$. Therefore $E$ draws ce $+C E$ coulombs out of one side of Fig. $p 3$ and forces it into the other side, and from this the total capacity of the arrangement is easily found.

4. Two condensers of which the capacities are $C=$ Io microfarads and $c=2$ microfarads are connected in series as shown in Fig. p4. What is the combined capacity of the arrangement? Ans. I.67 microfarads. 


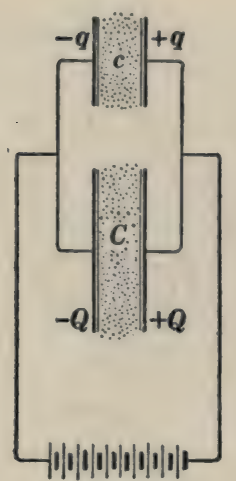

Fig. $p 3$.

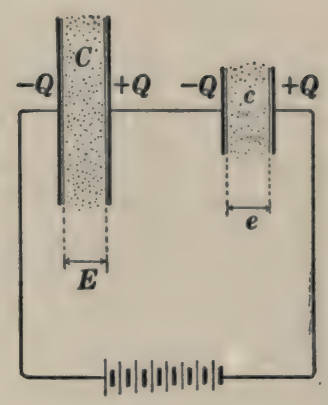

Fig. $p 4$.

Note.-An electromotive force $E+e$ will cause a certain amount of charge $Q$ to circulate around the circuit in Fig. $p 4$; and $Q=C^{\prime}(E+e)$ where $C^{\prime}$ is the combined capacity of the two condensers in series. But $Q=C E$ and $Q=c e$, and, if we substitute the values of $E$ and $e$ from these equations in $Q=C^{\prime}(E+e)$, we get an equation from which $Q$ cancels out and which can be solved to give $C^{\prime}$ in terms of $C$ and $c$.

5. An electromotive force acting on a condenser increases at a uniform rate from zero to Ioo volts during an interval of 0.005 of a second. The capacity of the condenser is 20 microfarads. Find the value of the current during 0.005 of a second. Ans. 0.4 ampere.

6. An alternating electromotive force which is represented by the ordinates of the zigzag line in Fig. p6 acts on a circuit which

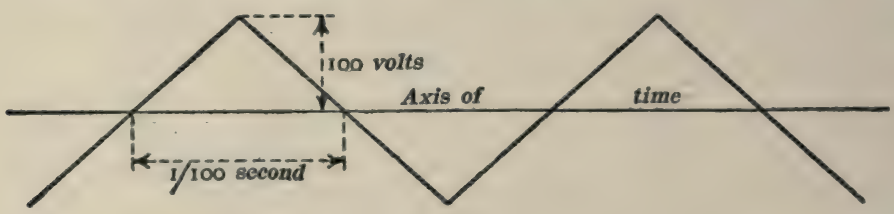

Fig. $p 6$.

contains a condenser. The resistance of the circuit is negligible, and the capacity of the condenser is 20 microfarads. Plot the curve of which the ordinates represent the successive instantaneous values of the current. 
7. A condenser is to be built up of sheets of tin foil 12 centimeters $\times$ I5 centimeters. The overlapping portions of the sheets are $\mathbf{I} 2$ centimeters $\times \mathbf{I} 2$ centimeters. The sheets are separated by leaves of mica 0.05 centimeter thick. How many mica leaves and how many tin foil sheets are required for a onemicrofarad condenser? Assume the inductivity of the mica to be equal to 6 . Ans. Mica, 655; tin foil, 656 .

8. A condenser consists of two flat plates each one meter square and at a distance of 0.5 centimeter apart. A rotating device is arranged to charge this condenser by connecting it to a 15 volt battery, and discharge it through a galvanometer 50 times each second, and the galvanometer deflection shows an average current of $1.33 \times 1 \mathrm{IO}^{-6}$ amperes. Find the capacity of the condenser and calculate the value of the factor $G$ in equation (I) of Art. 79. Ans. $884 \times 10^{-16}$.

Note.-A rotating device for making connections as specified in the problem is called a secohmmeter.

9. A condenser is made of two flat metal plates separated by air. Its capacity is 0.003 microfarad. Another condenser has plates twice as wide and twice as long. These plates are separated by a plate of glass (inductivity 5) which is four times as thick as the air space in the first condenser. What is the capacity of the second condenser? Ans. o.or 5 microfarad.

I0. Two metal plates, Ioo centimeters $\times$ Ioo centimeters, are separated by 2 centimeters of air. This condenser is charged by a battery having an electromotive force of 2,000 volts. What is its energy in joules? Ans. 0.000884 joule.

II. A flat glass plate, inductivity 5 , size 100 centimeters $\times$ Ioo centimeters $\times 2$ centimeters, is slid between the metal plates specified in problem Io, the battery being left connected to the metal plates. What is the energy of the condenser after the glass is in place? Ans. 0.00442 joule.

I2. The 2,000-volt battery is disconnected from the metal plates specified in problem I I after the glass is in place, and the 
metal plates are thoroughly insulated. The glass plate is then withdrawn, the whole charge being left on the metal plates. What is the electromotive force between the metal plates after the glass plate is withdrawn? How much has the energy been increased by withdrawing the glass? How much force was necessary to withdraw the glass, ignoring friction, weight, etc.? Assume that the glass is withdrawn sidewise, not cornerwise. Ans. 10,000 volts, 0.01768 joule, 1,768 dynes.

Note.-The increase of energy of the condenser is equal to the mechanical work done in withdrawing the glass plate.

13. The air condenser specified in problem ro is charged with 2,000 volts, the battery is disconnected and the metal plates are then moved to a distance 3 centimeters apart, charge on the plates remaining unchanged. What is the electromotive force between the plates after the movement? What is the increase of energy due to the movement? How much force was necessary to produce the movement, ignoring friction, weight, etc.? Ans. 3,000 volts, 0.000442 joule, 4,420 dynes. 


\section{CHAPTER VII.}

ELECTRIC FIELD.

83. The electric field.-When a momentary electric current flows through an open circuit, certain important effects are produced in the gap which breaks the circuit, and in order that these effects may be observed, a very high voltage machine must be used. The most convenient device for generating this high voltage is the influence electric machine. The two small black circles in Fig. 89 represent two metal balls which have been

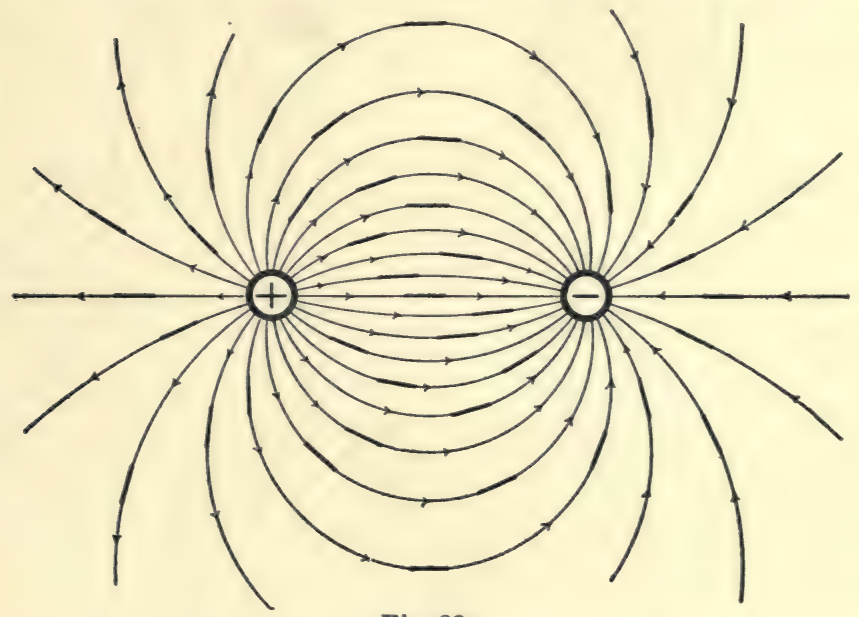

Fig. 89.

charged by drawing a momentary electric current out of one ball and forcing it into the other ball by an influence machine. When an ordinary wooden tooth pick, suspended by a fine thread, is placed in the region between the metal balls, the tooth pick points in a definite direction, at each point, very much as a magnet needle points in a definite direction, at each point, when it is placed between magnet poles. The short black lines in Fig. 89 represent the various positions of the tooth pick. 
The behavior of the tooth pick shows that the whole region surrounding the charged metal balls in Fig. 89 is in a peculiar condition, and this region is called an electric field. The direction of the electric field at each point is indicated by the suspended tooth pick, and lines drawn through the electric field so as to be at each point in the direction of the field at that point are called the lines of force of the electric field.

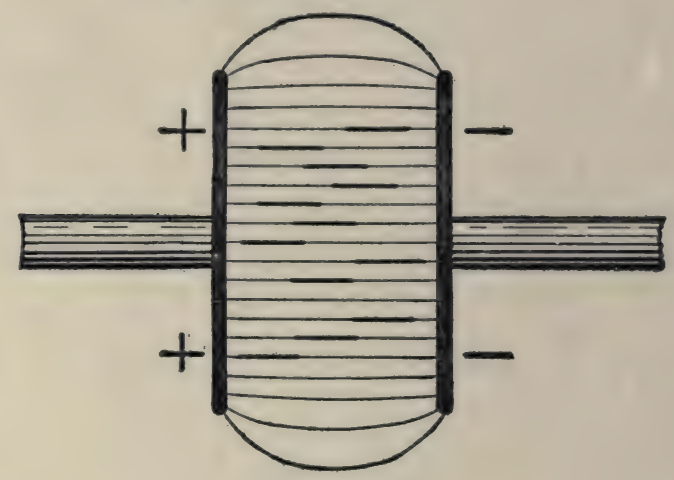

Fig. 90.

Figure 90 shows the lines of force of the electric field between two charged flat metal plates. The short black lines represent the positions of a suspended tooth pick. The lines of force in the region between the plates are straight lines, and the electric field is said to be uniform. The electric field between the charged balls in Fig. 89 is non-uniform.

84. Intensity of electric field.-It is permissible to adopt arbitrarily the ratio $E / x$ as a measure of the intensity of the uniform electric field between parallel flat metal plates, where $E$ is the electromotive force between the plates and $x$ is the distance between the plates. It is desirable, however, to base the definition of electric field intensity upon some observable effect of the field as in the following discussion.

Two metal plates $A$ and $B$, Fig. 9r, are connected to an electric machine giving a high electromotive force $E$ (the electric 
machine is represented in Fig. 9I as a battery). A small metal ball $b$ is suspended between the plates $A$ and $B$ by a silk thread, and if the ball $b$ is started it continues to vibrate back and forth from plate to plate. Regarding the behavior of the ball the following statements may be made:

(a) Work evidently is done to keep the ball $b$ oscillating back and forth, and this work is evidently done by the battery.

(b) The only way the battery can do work is by continuing to draw charge out of one plate and push it into the other plate. It is evident

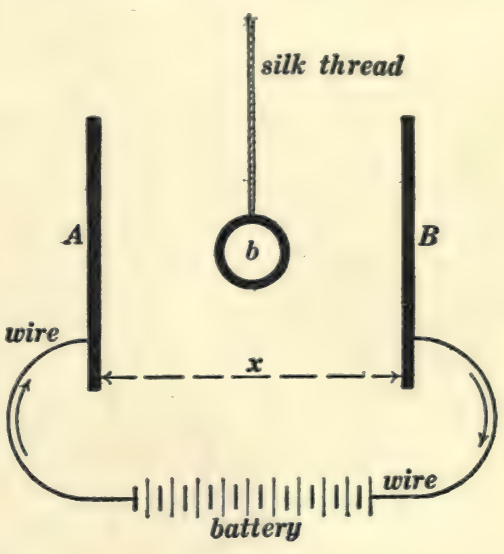

Fig. 91. therefore that the ball carries charge back and forth between the plates.

(c) The successive movements of the ball are similar, and therefore if the ball carries charge at all it must carry a definite amount each time it moves across. Let this definite amount of charge be represented by $q$; this charge is positive when the ball moves from $A$ to $B$, and negative when it moves from $B$ to $A$. At each movement of the ball the battery supplies the definite amount of charge $q$, drawing it out of plate $B$ and pushing it into plate $A$. Therefore, at each movement of the ball, the battery does an amount of work $E q$ according to equation (I) of Art. 80 .

(d) Let $F$ be the average mechanical force acting on the ball $b$ while it is being pulled across from plate to plate. If the ball is very small this force $F$ does not vary as the ball moves across, and if the ball is very small it moves the distance $x$ in travelling from plate to plate. Therefore $F x$ is the amount of work done on the ball while it moves from plate to plate. 
(e) The work $E q$ done by the battery during one movement of the ball is equal to the mechanical work $F x$ done on the ball, therefore we have $F x=E q$, or:

$$
F=\frac{E}{x} \cdot q
$$

Any region in which a charged body is acted upon by a force which depends upon the charge on the body and which does not exist when the body is not charged, is called an electric field. Thus the region between $A$ and $B$ in Fig. $9 \mathrm{I}$ is an electric field because the charged ball $b$ is acted upon by the force $F$.

The force $F$ with which an electric field pulls on a charged body placed at a given point in the field is proportional to the charge $q$ on the body so that we may write:

$$
F=e q
$$

in which $e$ is the proportionality factor, and it is called the intensity of the electric field at the given point.

From equations (I) and (2) we get:

$$
e=\frac{E}{x}
$$

That is to say, the intensity of the electric field between the plates in Fig. 9I is equal to the electromotive force between the plates divided by the distance between the plates.

Direction of electric field at a point.-The direction of an electric field at a point is the direction in which the field would pull on a positively charged body placed at that point.

Tension of the lines of force in an electric field.-Two oppositely charged metal plates attract each other as stated in Art. 75. Thus the oppositely charged plates in Fig. 90 attract each other. This attraction may be thought of as due to a tension of the lines of force; that is, the lines of force may be thought of as if they were filaments of rubber stretching from plate to plate and pulling the plates towards each other. 
If the lines of force in an electric field are like stretched filaments of rubber one would expect the lines of force to pull outwards on every part of the surface of a charged body. In fact, each part of the surface of a charged body is pulled outwards by the surrounding electric field. This outward pull may be beautifully shown by pouring melted rosin in a thin stream from a metal ladle which is supported by an insulated handle and connected to one terminal of an electric machine. The lines of force which emanate from the lip of the metal ladle pull the melted rosin into extremely fine jets which shoot straight outwards from the lip. These jets congeal in the form of excessively fine fibers which float about in the air.

85. Definition of electric flux in air.-Consider an area $a$ at right angles to an electric field of intensity $e$. The product $a e$ is called the electric flux across the area. Compare this with the definition of magnetic flux in Art. 25. If $a$ is expressed in square centimeters and $e$ in volts per centimeter or abvolts per centimeter, it is evident that ae is expressed in volts $\times$ centimeters or in abvolts $\times$ centimeters, respectively. Therefore, in what follows, electric flux will always be expressed in volt-centimeters or in abvolt-centimeters.

86. Amount of electric flux which emanates from $q$ coulombs of electric charge.-Let us consider the simplest example of electric field, namely, the field between two oppositely charged flat metal plates as shown in Fig. 90.

(a) This field is uniform, that is, the lines of force are straight and parallel.

(b) The intensity of the field is $\frac{E}{x}$, and this expression for the field intensity holds true whatever the nature of the dielectric, whether it be air, or oil or glass.

(c) The electric field is to be thought of as directed away from the positively charged plate and towards the negatively charged plate; therefore: 
(d) One may think of electric flux going out from the positively charged plate and as coming into the negatively charged plate.

(e) One of the most important theorems relating to electric field is that the amount of electric flux which goes out from a positive charge or which comes in to a negative charge is proportional to the amount of the charge. This theorem is due to Gauss. We will establish the theorem only for the case of oppositely charged flat metal plates.

Gauss's theorem for oppositely charged flat metal plates with air between them.-According to Art. 79 the capacity of a parallel-plate condenser with air as the dielectric is:

$$
C_{\text {In tarads }}=\frac{\mathrm{I}}{B} \cdot \frac{a}{x}
$$

where $\frac{\mathbf{I}}{B}$ is written for the constant $G$ so that the value of $B$ is:

$$
B=\mathrm{I} .13 \mathrm{I} \times 10^{13}
$$

where $a$ is the area of one of the plates in square centimeters, and $x$ is the thickness of the air layer in centimeters.

Let $E$ be the electromotive force between the plates in volts. Then the positive charge on one plate (or the negative charge on the other plate) in coulombs is $q=C E$. Therefore, using the value of $C$ from equation (I), we have:

$$
q=\frac{\mathbf{I}}{B} \cdot \frac{E}{x} \cdot a
$$

But $\frac{E}{x}$ is the intensity of the electric field between the plates in volts per centimeter, and therefore $\frac{E}{x} \cdot a$ is the electric flux from plate to plate, according to Art. 85. Consequently the electric flux going out from $+q$ coulombs or the electric flux coming in to $-q$ coulombs is $B q$ volt-centimeters.

Gauss's theorem for oppositely charged flat metal plates with any dielectric between them.-According to Art. 79 the capacity of a parallel-plate condenser with any dielectric is: 


$$
C_{\text {In tarads }}=\frac{\mathbf{I}}{B} \cdot k \cdot \frac{a}{x}
$$

where $B, a$ and $x$ are the same as in equation (I), and $k$ is the inductivity of the dielectric.

As before, let $E$ be the electromotive force between the plates; then:

$$
g=\frac{\mathbf{I}}{B} \cdot k \cdot \frac{E}{x} \cdot a
$$

But $\frac{E}{x}$ is the electrical field intensity or the electrical stress in the dielectric, and if we define $k \cdot \frac{E}{x}$ as the electrical flux density in the dielectric, then $k \cdot \frac{E}{x} \cdot a$ will be the total electric flux from plate to plate. Consequently the electric flux going out from $+q$ coulombs or the electric flux coming in to $-q$ coulombs is $B q$ volt-centimeters, where $B$ is a constant whose value is given in equation (2). This is Gauss's theorem, and although it has been established for a very special case, it is entirely general; $B q$ volt-centimeters of electric flux always goes out from $+q$ or comes in to $-q$ coulombs of charge.

87. Electric field intensity and electric flux density. Mechanical, magnetic and electric analogies.-Let $e$ be the intensity of an electric field at a point in a dielectric of which the inductivity is $k$; then

$$
F=k e
$$

where $F$ is the electric flux density at the point.

\section{Magnetic and Electric Parallel.}

$$
\mathscr{B}=\mu \mathscr{H}
$$

Where $\mathscr{H}$ is intensity of magnetic field in gausses, $\mu$ is the permeability of the medium, and $\mathscr{B}$ is the magnetic flux density. See Arts. 54 and 57.

$$
F=k e
$$

Where $e$ is intensity of electric field in volts per centimeter $(E / x), k$ is the inductivity of the medium, and $F$ is the electric flux density in volt-centimeters per square centimeter. 


\section{Electrical Stress and Mechanical Stress.}

The stretching force per unit of sectional area of a rod is called the stress on the rod, and the elongation of unit length of the rod is called the strain; and the strain is proportional to the stress. That is:

$$
\text { strain }=j \times \text { stress }
$$

where $j$ is a constant.

This constant is the reciprocal of what is usually called the modulus of elasticity, and it is large in value for a substance like rubber which is greatly stretched by a moderate stress.
The intensity of an electric field in volts per centimeter (or the volts per centimeter in a layer of dielectric between metal plates) is frequently called electrical stress, and the electric flux density in the dielectric is frequently called the electrical strain. Therefore, from equation (I) we have: electrical strain $=k \times$ electrical stress The inductivity of a dielectric is analogous to the elastic constant of a substance (reciprocal of what is called modulus of elasticity).

88. Continuity of electric flux or of electric flux density. Electric stresses in plane layers of different dielectrics.-Figure 92 shows a layer of oil (inductivity $k_{0}$ ) and a layer of glass (inductivity $=k_{g}$ ) between two flat metal plates $A$ and $B$. The electromotive force between the plates is $E$ volts, the charges on the plates are $+q$ and $-q$ coulombs, and the thicknesses of glass and oil layers are $x$ centimeters and $y$ centimeters respectively as shown; and it is required to find the electrical stress in the glass and in the oil.

The electric flux $B q$ which goes out from $+q$ and comes into $-q$ is independent of the change of dielectric, that is, the same amount of electric flux crosses the oil and the glass, and therefore the electric flux density $F$ is the same in both.

Let $e_{o}$ be the electrical stress in volts per centimeter in the oil, and let $e_{g}$ be the electrical stress in the glass. Then $k_{o} e_{o}$ is the flux density in the oil and $k_{0} e_{0}$ is the flux density in the 
glass, and, since the flux density is the same in both, we have:

$$
k_{o} e_{o}=k_{g} e_{g}
$$

Now $e_{0}$ being the electric field intensity (volts per centimeter) in the glass it is evident that $e_{g} x$ is the total voltage across the glass. Similarly, $e_{0} y$ is the total voltage across the oil. Therefore the total voltage from plate to plate is $e_{g} x+e_{0} y$. That is:

$$
E=e_{0} x+e_{0} y
$$

When $E, k_{g}, k_{o}, x$ and $y$ are given, these two equations enable $e_{g}$ and $e_{0}$ to be calculated.

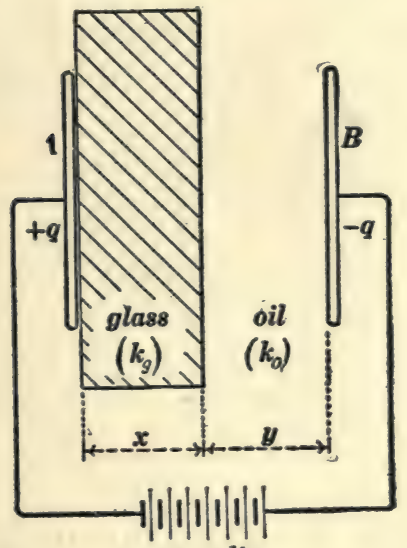

E volts

Fig. 92.

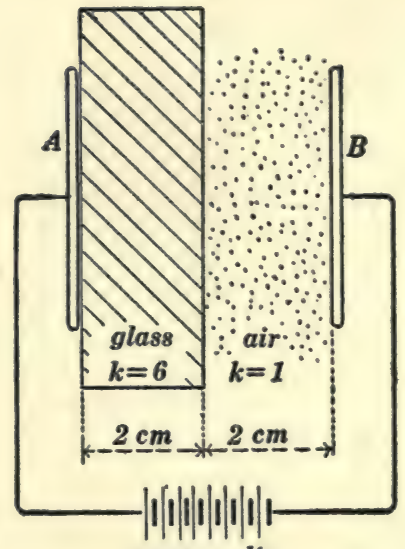

70000 volts

Fig. 93.

Example.-A layer of glass 2 centimeters thick $(k=6)$ and a layer of air 2 centimeters thick $(k=\mathrm{I})$ are subjected to a total electromotive force of 70,000 volts between parallel metal plates, as shown in Fig. 93, and from equations (I) and (2) we find the following: Electrical stress in the glass 5,000 volts per centimeter; Electrical stress in the air 30,000 volts per centimeter.

The presence of a layer of dielectric of large inductivity tirows an excess of stress on a layer of a dielectric of small inductivity. Thus with air alone between the metal plates in Fig. 93 (layer of air 4 centimeters thick), the electrical stress in the air would be 
I7,500 volts per centimeter; and the introduction of a glass plate 2 centimeters thick increases the stress on the remaining e-centimeter layer of air to 30,000 volts per centimeter.

This effect can be shown very beautifully by connecting two metal plates to a high-voltage transformer, and adjusting the plates to a distance such that the intervening air layer is barely sufficient to sustain the voltage. Then if a glass plate be introduced between the metal plates, the electrical stress in the remaining air will be increased sufficiently to break the air down at each reversal of the alternating voltage, as shown by the bluish luminosity of the air layer.

Magnetic analog.-Figure 94 represents a narrow air gap between the ends of two large magnetized iron rods. The

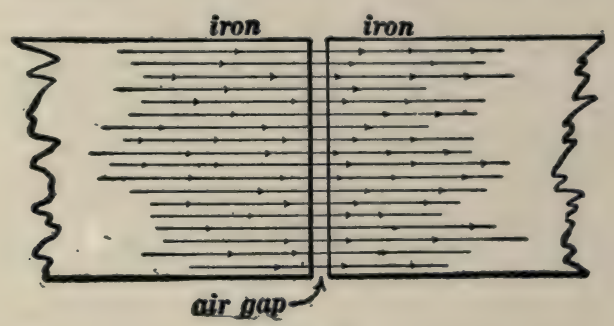

Fig. 94.

magnetic flux density $\mathscr{B}$ is the same in the air gap as in the iron (see Art. 54). The permeability of the iron is, say, I,000 and the permeability of the air is unity (see Art. 57). Therefore the value of $\mathscr{H}$ (the magnetic field intensity) is $\frac{\mathscr{B}}{\text { IOOO }}$ in the iron and $\frac{\mathscr{B}}{I}$ in the air. That is, the magnetic field intensity is $I, 000$ times as great in the low permeability air as in the high permeability iron just as the electric field intensity is 6 times as great in the air (having a low inductivity) as it is in the glass (having a high inductivity) in Fig. 93.

The concentration of the greater part of the voltage upon the air layer in Fig. 93 is exactly analogous to the concentration of 
the greater part of the magnetomotive force of a dynamo fieldwinding upon the air gap in the magnetic circuit; a small portion only of the magnetomotive force is required to force the magnetic flux through the highly permeable iron, and a large portion of the magnetomotive force is required to force the magnetic flux through the less permeable air layer. A small portion of the total voltage is required to force the electric flux through the highly inductive glass in Fig. 93 and a large portion of the voltage is required to force the electric flux through the less inductive air.

Mechanical analog of the above.-The glass and the air layers are in series in Fig. 93 and the electric flux density or electrical strain, or yield, is the same in both; whereas two mechanical elements have the same stress when they are in series; to get the same strain, or yield in two mechanical elements they must be in parallel. Thus Fig. 95 shows a column of steel and a column of rubber equally compressed between two bars $A$ and $B$ (the steel and rubber columns

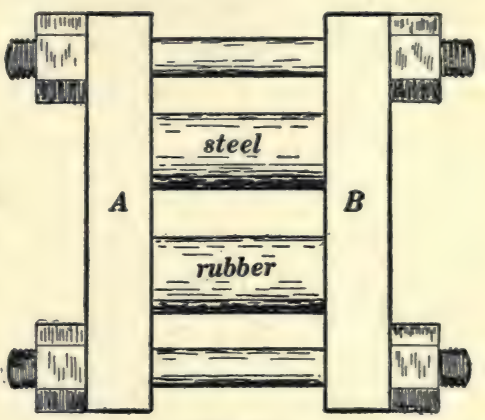

Fig. 95. are in parallel); the easily yielding rubber (high inductivity) supports only a small part of the compressing force, and the stiff steel (low inductivity) supports a large part of the compressing force.

Best shape of insulator.-When the electric flux passes from one insulating substance into another, an excessive concentration of electrical stress is likely to be brought about in the insulating substance of smaller inductivity, as above mentioned. Therefore, whenever possible, the surface of an insulator should be everywhere parallel to the lines of force of the electric field. 
Thus Fig. 96 shows a wire lying in a groove in a glass or porcelain support, and the surface of the porcelain is everywhere parallel to the lines of force of the electric field.

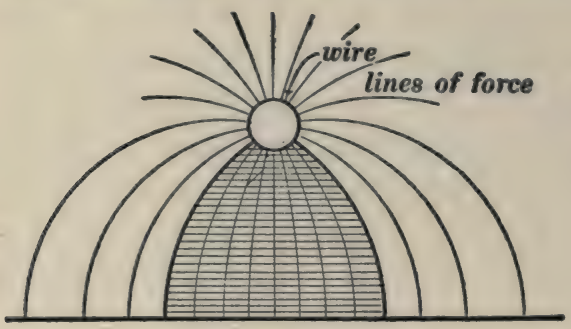

end view

Fig. 96.

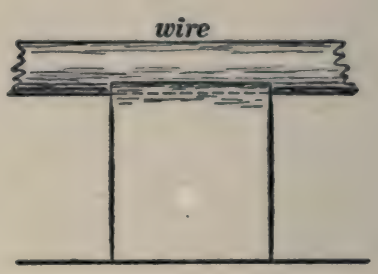

side view

89. Condenser type of insulating bushing.-An insulating bushing is a sleeve of insulating material which surrounds a wire where it passes through a conducting wall. Thus when a transformer is placed in an iron case, the wires which lead out from the transformer pass through insulating bushings in the cover of the transformer case. One difficulty in the design of such a bushing may be appreciated in terms of a mechanical analog as follows: When a very thick walled steel tube is subjected to internal pressure, the metal next to the bore is strained excessively before the outside metal comes into action to any great extent. Thus the full strength of the metal wall is not available for the

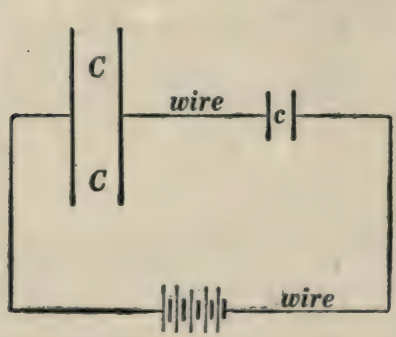

Fig. 97. withstanding of internal pressure. In the case of the bushing, the electrical stress in the insulating material next to the wire may be excessive, while the electrical stress in the outer portion of the bushing is very low, thus the full electrical strength of the bushing is not utilized.

The condenser type of insulating bushing is a bushing designed so as to equalize the electrical stress throughout the material of the bushing, and the principle 
which is made use of may be understood with the help of Fig. 97 which shows two condensers $c$ and $C$ connected in series and acted upon by an electromotive force. The momentary flow of current through the circuit charges both condensers equally, the amount of charge being, let us say, $q$ coulombs. Then the voltage across $c$ is $\frac{q}{c}$ and the voltage across $C$ is $\frac{q}{C}$, that is to say, the voltages across the respective condensers are inversely proportional to their respective capacities, and by varying the capacities (which can be done, say, by changing the sectional area of the dielectric) the total voltage can be subdivided between $c$ and $C$ in any way that is desired.

The design of the condenser type of bushing is shown in Fig. 98. The insulating material is laid on in layers of equal thickness, the length (parallel to the central rod) of each successive layer

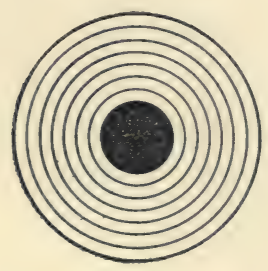

end view

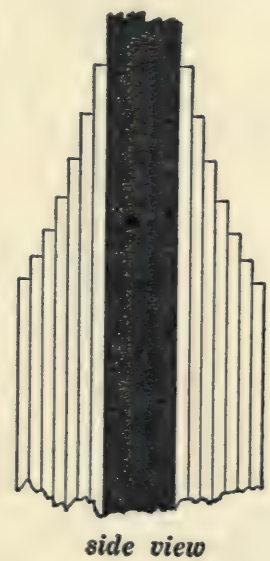

Fig. 98.

being reduced in proportion to the increase of its circumference, so that the area of each layer is the same. Furthermore a sheet of tin foil covers each successive layer of insulating material, and therefore these successive layers of tin foil together with the intervening layers of insulating material constitute a number of condensers of equal capacities all connected in series. In this 
way, the total voltage between the wire and the outside metal sheath is subdivided into equal parts in the various layers of insulating material.

90. Attraction of parallel charged plates.-Two parallel metal plates each $a$ square centimeters in area are placed at a distance of $x$ centimeters apart in an insulating fluid, such as air or oil, of which the inductivity is $k$. The charge on one plate is $+q$. coulombs and the charge on the other plate is $-q$ coulombs, and these charges remain constant, because the battery is disconnected and the plates are insulated. Let $F$ be the force with which the p'ates attract each other; then, if the plates be moved farther apart so that $x$ is increased to $x+\Delta x$, an amount of work equal to $F \cdot \Delta x$ will be done, and this work will all go to increase the energy of the charged condenser; therefore if we can calculate the increase of the potential energy of the condenser due to the movement $\Delta x$, we will arrive at an expression for the force $F$.

The energy of a charged condenser, expressed in terms of its capacity $C$ and charge $q$, is given by equation (6) of Art. 8I, namely:

$$
W=\frac{1}{2} \frac{q^{2}}{C}
$$

and the capacity of a parallel-plate condenser is:

$$
C=\frac{\mathrm{I}}{B} \cdot \frac{k a}{x}
$$

as explained in Art. 79. Therefore, substituting this value of $C$ in equation (I) we have:

$$
W=\frac{1}{2} \frac{B q^{2}}{k a} \cdot x
$$

Differentiating this expression with respect to $x$, we have:

$$
\Delta W=\frac{1}{2} \frac{B q^{2}}{k a} \cdot \Delta x
$$

* $\frac{Y}{B}$ is written for the constant $G$ of Art. 79 so that the value of $B$ is I.131 $\times 10^{13}$. 
This is the increase of potential energy of the condenser due to the movement $\Delta x$, and, as above explained, it is equal to $F \cdot \Delta x$. Therefore we have:

$$
F \cdot \Delta x=\frac{1}{2} \frac{B q^{2}}{k a} \cdot \Delta x
$$

or

$$
F=\frac{1}{2} \frac{B q^{2}}{k a}
$$

Now, $q$ being in coulombs, equation (3) gives $W$ in joules; so that $\Delta W$ in equation (4) is in joules. Therefore $F \cdot \Delta x$ is expressed in joules, and $F$ in equation (5) is expressed in joules per centimeter, one joule per centimeter being the force which will do one joule of work while the body on which it acts moves one centimeter in its direction. One joule per centimeter is equal to ten million dynes.

It is noteworthy that the force of attraction of two parallel charged plates due to given charges $+q$ and $-q$ on the plates is independent of the distance between the plates, provided that the plates are very large as compared to the distance between them.*

The charge $\pm q$ on the two plates is equal to $C E$, where $E$ is the electromotive force between the plates and $C$ is the capacity of the condenser formed by the two plates. Therefore, substituting the value of $C$ from equation (2), we have:

$$
q=\frac{\mathrm{I}}{B} \cdot \frac{k a}{x} \cdot E
$$

and substituting this value of $q$ in equation (5), we have:

$$
F=\frac{I}{2 B} \cdot \frac{k a E^{2}}{x^{2}}
$$

which gives the force of attraction of two plates in terms of the electromotive force between the plates. This equation gives the value of $F$ in joules per centimeter when $a$ is expressed in

\footnotetext{
* Equation (2) is true only when $x$ is small as compared with the size of the plates.
} 
square centimeters, $x$ in centimeters and $E$ in volts; $k$ being the inductivity of the fluid dielectric, and $B$ being equal to I.I3I $\times \mathrm{IO}^{13}$.

It is to be noted that the force of attraction of two parallel plates is inversely proportional to the square of the distance between the plates for a given value of $E$.

The absolute electrometer.-If an electromotive force $E$ to be measured is connected to two parallel metal plates in air, the value of $E$ in volts can be calculated with the help of equation (7), above, if $a$ and $x$ are known and if the force $F$ is measured; $k$ being equal to unity and $B$ being a known constant. A device arranged for measuring an electromotive force in this way is called an absolute electrometer because only mechanical and geometrical measurements need be made.

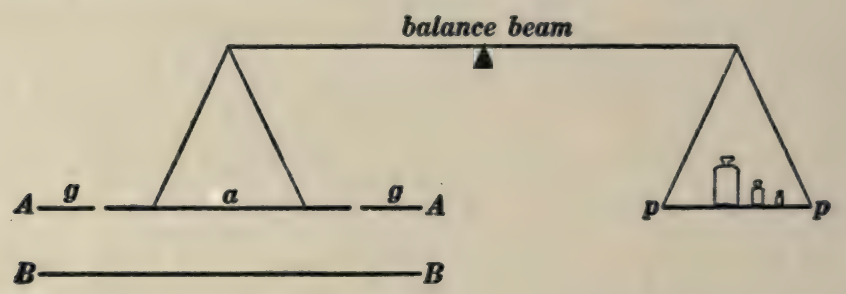

Fig. 99.

Figure 99 shows the essential features of an absolute electrometer. The two attracting metal plates $A$ and $B$ are horizontal, and the upper plate is in two parts, namely, a central circular part of area $a$ which is hung from one end of a balanced beam, and a surrounding part $g g$ which is supported rigidly and which is called a guard ring. The force with which the suspended portion $a$ is attracted by the lower plate is measured by placing weights on the pan $p p$.

Equation (7) is accurately true only when the attracting plates are large as compared with the distance $x$; but equation $(7)$ is accurately true for the central portion $a$ of the upper plate in Fig. 99. 
91. Energy and tension of the electric field in air.-It is a great help in the development of the theory of the electric field if one appreciates as fully as possible the various properties of the electric field. Thus equation (2) of Art. 84 expresses an important property of the electric field. Another interesting property of the electric field is that the lines of force tend to shorten, that is, the electric field is in a state of tension along the lines of force; and another interesting property is that the electric field stores energy. These two properties are here discussed for the electric field in air.

Energy of the electric field in air.-The energy of a charged condenser is:

$$
W=\frac{1}{2} C E^{2}
$$

according to Art. 8I. Substituting the value of $C$ from equation (2) of Art. 79, remembering that $k=\mathbf{I}$ for air, we get:

$$
W=\frac{\mathrm{I}}{2 B} \cdot \frac{a E^{2}}{x}=\frac{\mathrm{I}}{2 B} \cdot a x \cdot \frac{E^{2}}{x^{2}}
$$

but $a x$ is the volume $v$ of the region between the plates, and $\frac{E}{x}$ is the intensity $e$ of the electric field between the plates. Therefore equation (2) may be written:

$$
W=\frac{\mathbf{I}}{2 B} \cdot v \cdot e^{2}
$$

or, dividing both members by $v$, we get:

$$
\text { energy per unit volume }=\frac{I}{2 B} \cdot e^{2}
$$

Now the energy of a charged condenser resides in the electric field, and equation (4) shows that the energy of an electric field in air in joules per cubic centimeter is $\frac{I}{2 B} \cdot e^{2}$, where $e$ is the intensity of the field in volts per centimeter.

* The factor $\frac{I}{B}$ is written for $884 \times 10^{-16}$. 
Tension of the electric field in air.-Putting $k=\mathrm{I}$ and writing $e$ for $\frac{E}{x}$ in equation (7) of Art. 90 we get:

$$
F=\frac{\mathbf{I}}{2 B} \cdot a e^{2}
$$

Now the force $F$ which tends to pull the two plates together shows that the intervening electric field is in tension and the force per unit area is the measure of the tension $T$. Therefore, dividing both members of equation (5) by $a$, we get:

$$
T=\frac{\mathrm{I}}{2 B} \cdot e^{2}
$$

where $T$ is the tension in an electric field of which the intensity is $e$ volts per centimeter.

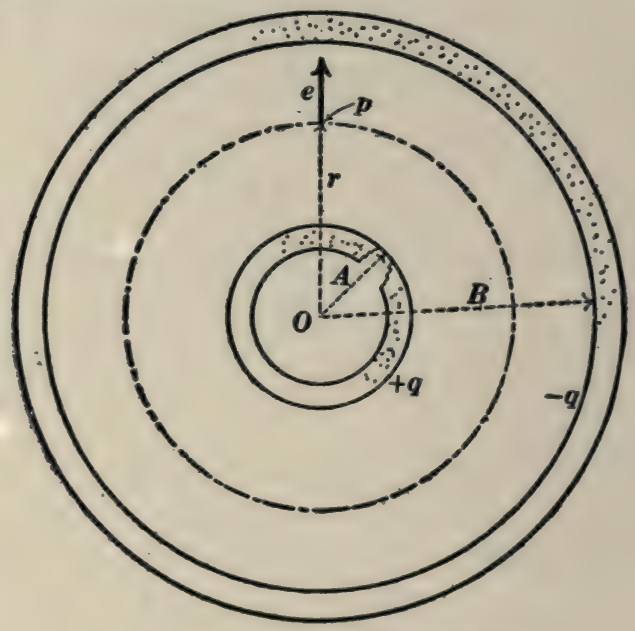

Fig. 100.

Equations (4) and (6) are here derived for a very special case but they give the energy per unit volume and the tension at a point in any electric field in air.

92. The electric field in the region between two concentric metal spheres.-A metal sphere of radius $A$ is surrounded by a hollow metal sphere of internal radius $B$ as shown in Fig. 100. 
The small sphere has a charge of $+q$ coulombs, and the inside surface of the outer sphere has a charge of $-q$ coulombs; and it is required to find the intensity of the electric field at any point $p$ distant $r$ from the common center $O$ of the two spheres. It is evident that the lines of force of the electric field are radial straight lines because of the entire symmetry of the arrangement. Therefore the electric field at $p$ is in the direction of the arrow $e$. Imagine a spherical surface of radius $r$ with its center at $O$. The area of this spherical surface is $4 \pi r^{2}$ and the electric field is everywhere at right angles to its surface and equal to $e$. Therefore the flux across the spherical surface is $4 \pi r^{2} e$, and according to Gauss's theorem this flux must be equal to $B q$ (see Art. 86). Therefore we have:

$$
e=\frac{B}{4 \pi} \cdot \frac{q}{r^{2}}
$$

The expression for $e$ in equation (I) does not depend upon the radius of either sphere in Fig. I0o. Therefore equation (I) gives the electric field intensity $e$ in volts per centimeter at any point at a distance of $r$ centimeters from the center of a sphere of any size over which $q$ coulombs of charge is uniformly distributed, provided the given sphere is surrounded by a hollow concentric metal sphere of any radius greater than $r$. Of course $r$ must be greater than the radius of the inner sphere.

93. Electric field due to a concentrated charge.-Imagine a charge of $q$ coulombs on a sphere of indefinitely small radius. Such an imagined charge is called a concentrated charge. It is evident from what is said above that equation (I) of Art. 92 gives the intensity $e$ of the electric field at a distance of $r$ centimeters from a concentrated charge of $q$ coulombs.

Attraction or repulsion of concentrated charges.-Consider two concentrated charges $q^{\prime}$ and $q^{\prime \prime}$ at a distance of $d$ centimeters apart. The electric field intensity at $q^{\prime \prime}$ due to $q^{\prime}$ is $e^{\prime}=\frac{B}{4 \pi} \cdot \frac{q^{\prime}}{d^{2}}$, and the force $F$ exerted on $q^{\prime \prime}$. by this field is equal 
to the product $e^{\prime} q^{\prime \prime}$ according to Art. 84. Therefore we have:

$$
F=\frac{B}{4 \pi} \cdot \frac{q^{\prime} q^{\prime \prime}}{d^{2}}
$$

where, according to Art. 84, $F$ is expressed in joules per centimeter. The force $F$ is the force with which the two concentrated charges attract or repel each other; it is a repulsion when $q^{\prime}$ and $q^{\prime \prime}$ are alike in sign, and it is an attraction when $q^{\prime}$ and $q^{\prime \prime}$ are opposite in sign.

The "electrostatic" unit of charge.-The amount of concentrated charge which will attract or repel an equal amount of concentrated charge with a force of one dyne at a distance of one centimeter is used as a unit of charge by many writers on electrical theory, and it is called the "electrostatic" unit of charge.

To find the value of the "electrostatic" unit of charge in coulombs substitute $q$ for $q^{\prime}$ and $q^{\prime \prime}$ in equation (I); write $10^{-7}$ for $F, \mathrm{I} . \mathrm{I} 3 \mathrm{I} \times 1 \mathrm{I}^{13}$ for $B$ and $\mathrm{I}$ for $d$; and solve for $q$. This gives

one "electrostatic" unit of charge $=3.33 \times 10^{-10}$ coulombs

94. Electric field in the region between two co-axial metal cylinders.-A metal cylinder of radius $A$ is surrounded by a hollow metal cylinder of which the inside radius is $B$ as shown in Fig. IоI, the space beiween $A$ and $B$ is filled with a dielectric of which the inductivity is $k$, the inside cylinder has $+q$ coulombs of charge pe: centimeter of length and the outside cylinder has on its inner surface $-q$ coulombs of charge per centimeter of length; and it is required to find the intensity $e$ of the electric field at a point $p$ at a distance of $r$ centimeters from the common axis $O$ of the two cylinders.

Imagine a cylindrical surface of radius $r$ with its axis a'ong $O$. The electric field $e$ at the surface of this cylinder is everywhere the same in value and everywhere at right angles to the surface. Let us consider a portion of length $l$ of the two cylinders and of the cylindrical surface of radius $r$. The area of this portion 
of the cylindrical surface is $2 \pi r l$ square centimeters, and the amount of electric flux which crosses it is $2 \pi r l \times k e$. But the amount of charge on $l$ centimeters of the inner cylinder is $l q$. Therefore, according to Gauss's theorem, we have:

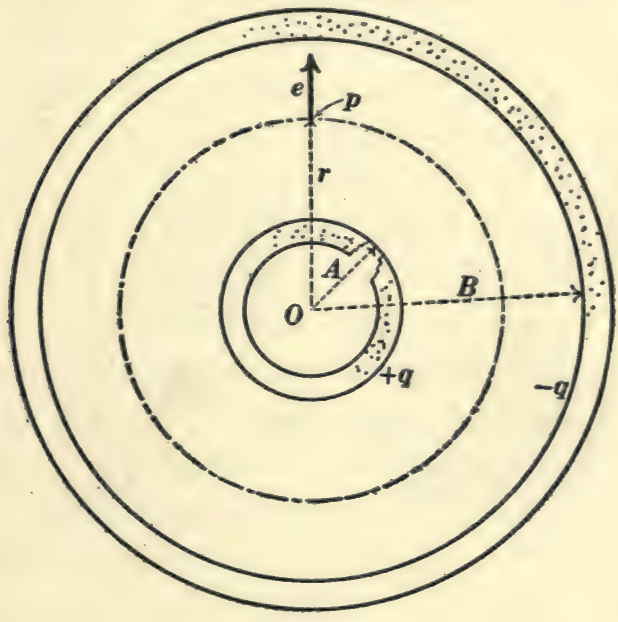

Fig. 101.

$$
2 \pi r l k e=B l q
$$

from which we get:

$$
e=\frac{B}{2 \pi} \cdot \frac{\mathbf{I}}{k} \cdot \frac{q}{r}
$$

For the simplest case, namely when the space between the cylinders is filled with air, then $k=\mathrm{I}$ and this equation becomes:

$$
e=\frac{B}{2 \pi} \cdot \frac{q}{r}
$$

Remark.-This equation also expresses the intensity $e$ of the electric field at a point $r$ centimeters from the axis of a long cylinder which is at a great distance from all other bodies, $q$ being the charge in coulombs on each centimeter of length of the cylinder. This is evident when we consider that equation (2) 
does not contain the radius of the outside cylinder so that this radius may be as large as you please.

95. Graded cable insulation.-Equation (I) of Art. 94 shows how the electrical stress $e$ is distributed throughout an ordinary electric cable which consists of a cylindrical wire core, a covering of rubber or other insulating material and a lead sheath. If $k$ is the same throughout the insulating material, then $e$ is much larger near the central wire where $r$ is small, than near the sheath where $r$ is large; but if it were possible to apply the insulating material in layers with the value of $k$ decreasing as $r$ increases so as to keep the product $k r$ constant, then the value of $e$ would be the same throughout the insulating material of the cable and every portion of the insulating material would carry its full share of the total stress (voltage) between wire core and sheath. Such a gradation of $k$ is however, impracticable, but it is practicable to apply the insulating material in two or three layers, the inside layer having large inductivity, the middle layer having medium inductivity, and the outer layer having small inductivity. Such a cable insulation is called a graded cable insulation.

\section{Magnetic and electric parallels. Magnetic permeability} is analogous to electric inductivity.

The lines of force of a magnetic field converge upon and pass through an iron rod as shown in Fig. I02.
The lines of force of an electric field converge upon and pass through a glass rod as shown in Fig. 103.

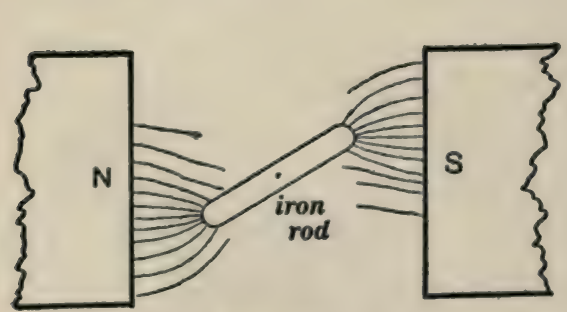

Fig. 102.

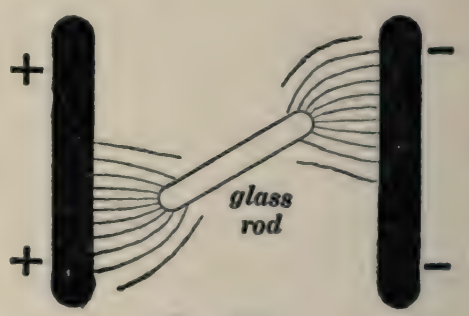

Fig. 103. 
The iron rod in Fig. I02, if free to turn, oscillates back and forth through an equilibrium position parallel to the field.

An iron plate is drawn into the intense magnetic field between two opposite magnet poles as shown in Fig. I04.

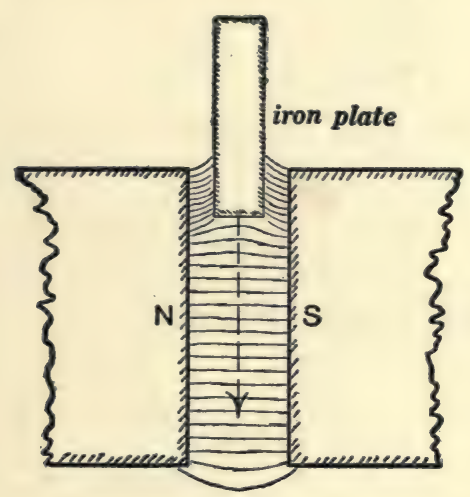

Fig 104.
The glass rod in Fig. 103, if free to turn, oscillates back and forth through an equilibrium position parallel to the field.

A glass plate is drawn into the intense electric field between two charged metal plates as shown in Fig. I05.

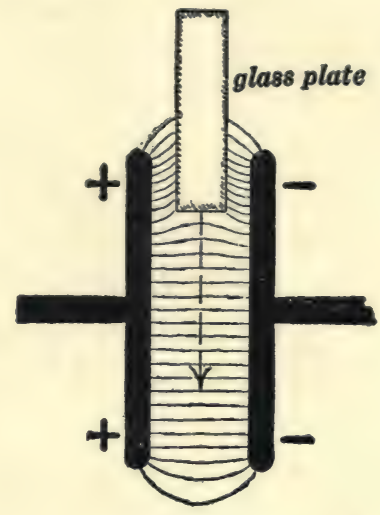

Fig. 105.

97. Dielectric hysteresis.-The most prominent kind of dielectric hysteresis is a kind which is closely analogous to what is technically called elastic lag in mechanics. For example, glass when subjected to a mechanical stress takes on a certain amount of strain (deformation) quickly, after which the strain increases slowly for a time; and when the stress is removed a remnant of the strain persists for a time.

A condenser is charged. The coatings of the condenser are then momentarily connected by a wire which discharges the condenser. The wire is then disconnected, and the con- is then momentarily released.
A rubber tube is stretched. (This stretch corresponds to the electrical strain of the dielectric of a charged condenser.) The end of the rubber tube 
denser is left standing on open circuit. The coatings of the condenser are then again connected and a second slight discharge is obtained.
(This corresponds to the discharge of the condenser.) The end of the tube is then again clamped fast in what seems to be its equilibrium position. (This corresponds to the disconnecting of the wire from the discharged condenser.) After a time the end of the tube is again released and a second slight shortening of the tube takes place. (This corresponds to the second discharge which is obtained from the condenser.)

98. Concentration of electrical stresses by points.

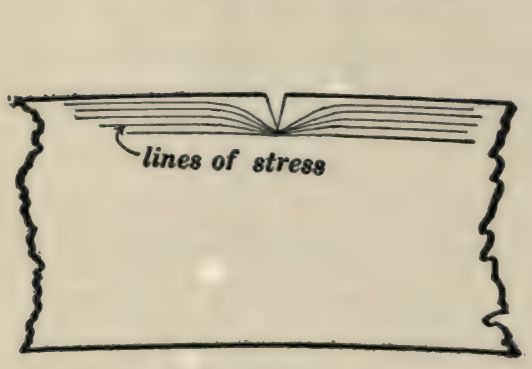

Fig. 106.

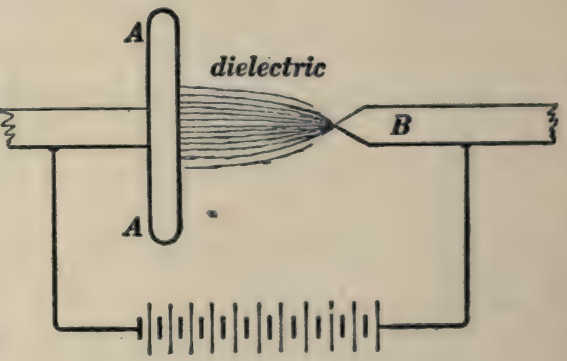

Fig. 107.

Fig. I06 shows the lines of mechanical stress passing around the bottom of a sharp groove in a bent bar. The stress is very greatly concentrated near the bottom of the groove, and the groove deepens by the formation of a crack. The stress is then concentrated

Figure 107 shows the lines of electrical stress between a flat plate and a point. The stress is very greatly concentrated near the point and if the voltage is great enough a rupture of the dielectric begins at the point. The line of incipient rupture is an electrical con- 
at the edge of this crack, and the crack extends farther and farther until the bar is broken in two.

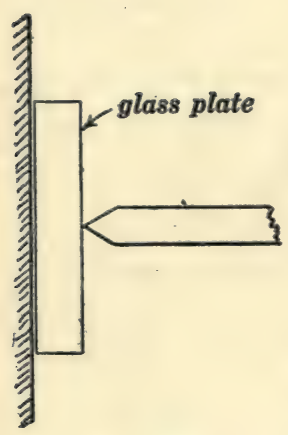

Fig. 108.

Figure 108 shows a hardened steel point pressed against a small glass plate. The concentration of the mechanical stress at the point is so great that a comparatively small force is sufficient to break the plate. ductor, as if it were an extension of the metal point. Therefore the electrical stress is concentrated at the end of the incipient rupture so that the rupture extends farther and farther until the spark reaches from $A$ to $B .^{*}$

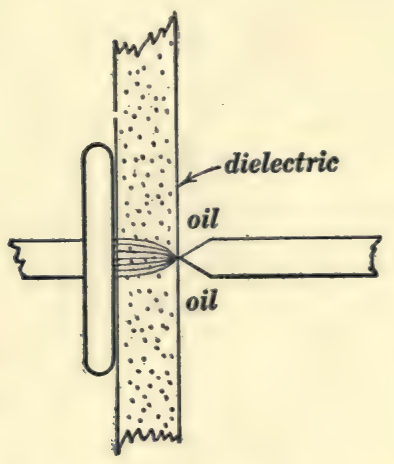

Fig. 109.

Figure I09 shows a plate of glass or other dielectric between a flat metal plate and a metal point. The concentration of the electrical stress near the point is so great that a comparatively small electromotive force between metal plate and metal point is sufficient to puncture the dielectric.

* This purely mechanical view of the formation of a spark is inadequate when one goes into a minute study of the phenomena of electric discharge. The point of view which has shown itself to be the most useful is the point of view which is involved in the electron theory which is briefly discussed in Chapter X. 


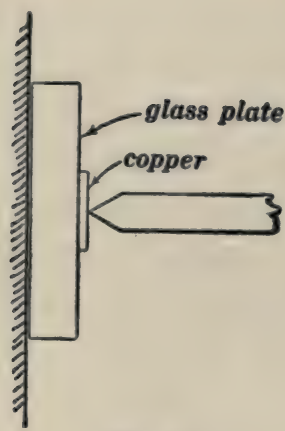

Fig. 110.

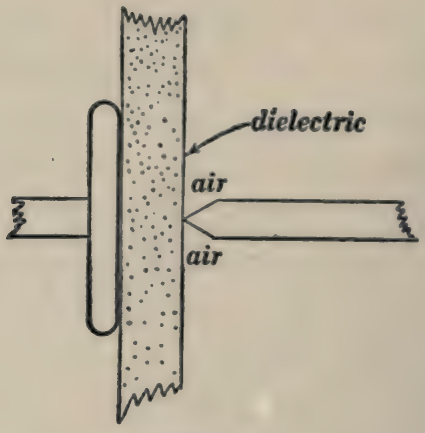

Fig. 111.
Figure IIo is the same as Fig. I08 except that a soft bit of copper is placed under the steel point. In this case the copper gives way, and the stress is not so greatly concentrated. In fact it is the same as if one were pushing a flat ended tool against the glass, when a very great force is required to break the glass.
Figure III is the same as Fig. 109 except that the point is surrounded by air instead of oil. The electricstrength of the air is less than that of the layer of dielectric, and therefore the air breaks down electrically and prevents an extreme concentration of electrical stress near the point. The result is very much as if the plate of dielectric were placed between two flat metal plates, when a very arge voltage is required to puncture the dielectric. 


\section{PROBLEMS.}

I. A very small ball $b$ weighing o.I gram and suspended by a very fine fiber of silk stands as shown in Fig. $p \mathbf{I}$. What is the charge on $b$ ? Ans. $1.78 \times \mathrm{IO}^{-9}$ coulombs.

Note.-Take 980 dynes as the weight of one gram.

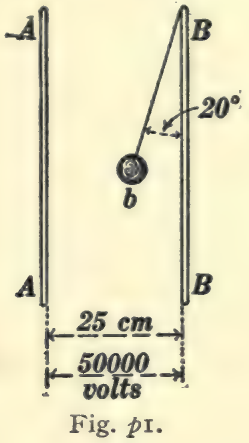

2. Find the maximum charge that can be held on a sphere 200 centimeters in radius, the sphere being surrounded by air of which the dielectric strength is, say, 33,000 volts per centimeter. Ans. 0.001469 coulomb.

Note.-The maximum electric field intensity at the surface of the sphere being known, the corresponding flux from the sphere and charge on the sphere can be calculated.

3. The sphere of the previous problem is surrounded by a dielectric of which the strength is 60,000 volts per centimeter and of which the inductivity is 2.5. Find the maximum charge that can be held on the sphere. Ans. 0.00667 coulomb.

4. Two flat metal plates 7 centimeters apart are separated by a layer of oil 3 centimeters thick (inductivity 2.00), and a layer of glass 4 centimeters thick (inductivity 5). An electromotive force of 20,000 volts is connected to the metal plates. Find the electrical stress in the oil and in the glass. Ans. 4,347 volts per centimeter in the oil; 1,739 volts per centimeter in the glass.

5. Calculate the force of attraction of two parallel flat metal plates 30 centimeters square and one centimeter apart with air 
between when an electromotive force of 10,000 volts is connected to the plates. Ans. 0.00796 joule per centimeter or 79,600 dynes.

6. What would the force of attraction of the plates of problem 5 be with an electromotive force of 100 volts? Ans. 7.96 dynes.

7. The space between the plates in problem 5 is filled with oil of which the inductivity is 2.5. What is the attraction of the plates? Ans. 199,000 dynes.

8. The electric field intensity at a point 200 centimeters from the center of a uniformly charged sphere, say, 50 centimeters in radius, is 250 volts per centimeter. The sphere is surrounded by air. What is the charge on the sphere? Ans. 0.0000 I I coulomb. 


\section{CHAPTER VIII.}

THE IDEA OF POTENTIAL AND ITS USE IN THE STUDY OF THE ELECTRIC FIELD.

99. Definition of velocity potential of a moving fluid.-In the study of a moving fluid it is sometimes* helpful to imagine a sort of a "hill" whose "slope" at each point is equal to the fluid velocity at that point, and the "height" of this imagined hill at a point is called the velocity potential of the moving fluid at that point. Let $\Delta h$ be the change of height of the potential hill corresponding to the distance $\Delta x$ measured along the base of the hill. Then $\frac{\Delta h}{\Delta x}$ is the slope of the hill, and according to the above definition this slope is equal to the velocity of the fluid in centimeters per second. Therefore $\frac{\Delta h}{\Delta x}$ is expressed in centimeters per second, and if $\Delta x$ is expressed in centimeters it is evident that $\Delta h$ must be expressed in centimeters squared per second. It is evident, therefore, that the velocity potential of a moving fluid is not a geometrical hill. Indeed, when a fluid is moving in three dimensions the entire region occupied by the fluid is, as it were, the "base" of the potential hill. A clear idea of velocity potential in this case may be reached by imagining the temperature in a region to vary from point to point in such a way that the temperature gradient or temperature slope at each point may represent the fluid velocity at that point in magnitude and in direction. Then the temperature at each point represents the height of the potential hill at that point.

Definition of electric potential.-In the study of electric field distribution it is sometimes helpful to imagine a sort of hill whose

* The simplification of the mathematical treatment of fluid motion by the use of the idea of velocity potential is exemplified on page 246 of Franklin, MacNutt and Charles's Calculus, published by the authors, South Bethlehem, Pa., I913. 
slope at each point is equal to the intensity of the electric field at that point. The height of this imagined hill at a point is called the electric potential at that point. Thus the fine curved lines with arrow heads in Fig. II 2 represent the lines of force of the

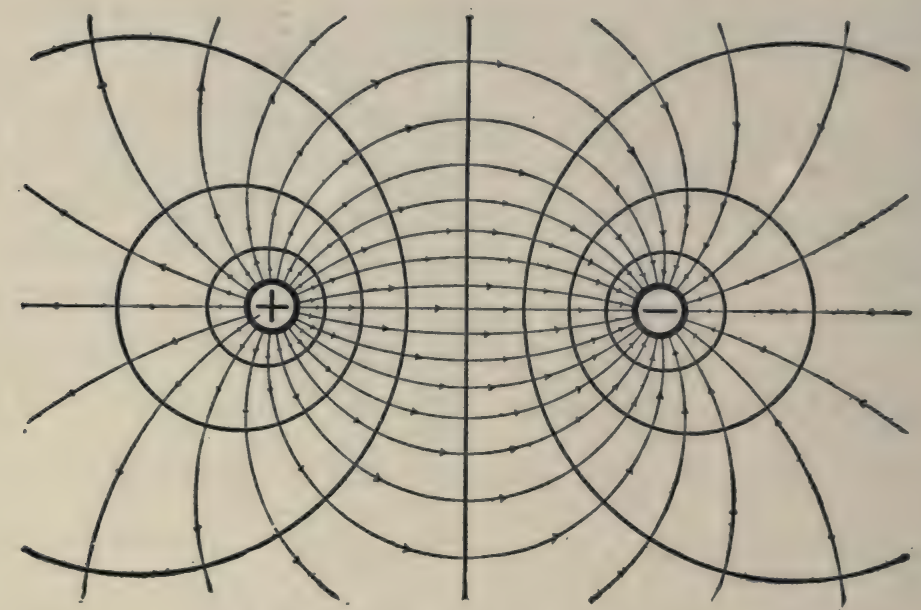

Fig. 112.

electric field between two parallel oppositely charged metal cylinders, the axes of the cylinders being perpendicular to the plane of the paper. The lines of force are the slope lines of the potential hill, and the heavy lines are the contour lines or lines of equal level on the potential hill. The slope lines are everywhere at right angles to the contour lines. The height of the potential hill at each point is expressed in volts.

Equipotential surfaces.-Each of the contour lines in Fig. II2 represents a surface perpendicular to the plane of the paper, the lines of force of the electric field cross these surfaces everywhere at right angles, and each surface is everywhere at the same potential, that is to say, the value of the potential in volts or abvolts is the same at every point on each surface. Such a surface is therefore called an equipotential surface.

A clear idea of what is represented in Fig. II 2 may be obtained by imagining that the positively charged cylinder is at a high 
temperature and the negatively charged cylinder is at a low temperature, the intervening region being filled with a heat conductor. Then the heat-flow through the surrounding region would be along the fine lines with arrow heads, and the heavier lines would be lines of equal temperature. The heavier lines represent surfaces at right angles to the plane of the paper, and each of the surfaces so represented would be an isothermal surface.

Let $V$ be the value of the electric potential at a point, then $\frac{d V}{d x}$ is the gradient or slope of $V$ in the direction of the $x$-axis of reference, and therefore, in accordance with the above definition of potential, we have:

$$
\begin{aligned}
& \frac{d V}{d x}=X, \\
& \frac{d V}{d y}=Y, \\
& \frac{d V}{d z}=Z,
\end{aligned}
$$

where $X, Y$ and $Z$ are the components (parallel to the $x, y$ and $z$-axes respectively) of the electric field at a point. Also it is evident that $d V=X \cdot d x$, that is the increment of potential along $d x$ is found by multiplying the $x$-component of the electric field by $d x$.

A clear understanding of electric potential may be obtained by considering the electric field between the two plates in Fig. 90 or Fig. 91. Let $E$ be the electromotive force between the plates, then $E / x$ is the field intensity or slope of the potential hill, and if we multiply this slope, $E / x$, by the distance $x$ between the plates we get the total rise $E$ of the potential hill from one plate to the other. That is to say, the electromotive force between any two points is the difference in level of the potential hill at these two points or simply the potential difference between these two points. Let $q$ coulombs be an amount of charge which is transferred from one point to another, and 
let $E$ volts be the electromotive force or potential difference between these points Then $E q$ joules is the amount of work done on the charge by the electric field. Of course the charge may be thought of as transferred along a wire.

The actual physical conditions in an electric field are completely specified when the intensity of the field is everywhere given. Therefore the potential hill can be determined only in that its slope can be everywhere known. It is evident, however, that a hill is not completely determined when its slope is everywhere known; its height at some point must be given. Therefore a complete specification of the electric potential in any given case involves an arbitrary and physically meaningless choice of a region of zero potential. When the region of zero potential has been chosen, then the electric potential at any point $p$ is the electromotive force $E$ between that point and the arbitrarily chosen region of zero potential; and if a charge of $q$ coulombs is carried from $p$ to the region of zero potential, the work done on the charge by the electric field will be $E q$ joules, or the work done per unit of positive charge will be equal to $E$. Therefore the electric potential at a point $p$ is equal to the work done by the electric field on one unit of positive charge which is carried from $p$ to the region of zero potential.*

I00. Potential values in the neighborhood of a uniformly charged sphere.-As an example of the use of the idea of potential, let us determine the distribution of potential in the neighborhood of a uniformly charged sphere $S$, Fig. II3; the charged

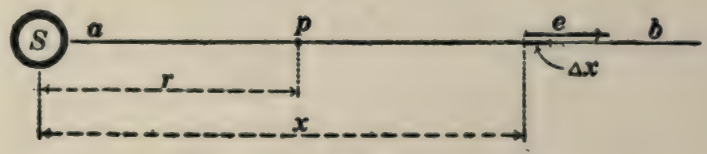

Fig. 113.

sphere being at a great distance rrom all surrounding objects. Let us choose the region infinitely remote from $S$ as the region

* A very good discussion of the mathematical theory of potential is given on pages 210-253 of Franklin, MacNutt and Charles' Calculus. 
of zero potential. The problem is to determine the electric potential at any given point $p$ at a distance of $r$ centimeters from the center of the sphere, and to do this it is sufficient (according to the statement in Art. 99) to find how much work will be done by the electric field on one coulomb of positive charge which is carried from $p$ to an indefinitely great distance. The path over which the charge is carried is a matter of indifference; therefore let us choose the straight line $a b$ as the path over which the charge is to be carried.

The electric field intensity at the element $\Delta x$ due to the charged sphere is:

$$
e=\frac{B}{4 \pi} \cdot \frac{Q}{x^{2}}
$$

according to equation (I) of Art. 92, where $Q$ is the amount of charge on the sphere $S$. Suppose a body carrying $q$ coulombs is moved along $\Delta x$; the force exerted on this body is $F=q e$, according to Art. 84 , or using the above value of $e$, we have:

$$
F=\frac{B Q q}{4 \pi} \cdot \frac{\mathbf{r}}{x^{2}}
$$

and this force is in the direction of $e$ as shown by the arrow in Fig. I13. Now as the charge $q$ is carried along $\Delta x$ in the direction of the electric field $e$ an amount of work $\Delta W=F \cdot \Delta x$ is done by the electric field. Therefore, using the value of $F$ from equation (2), we have:

$$
\Delta W=\frac{B Q q}{4 \pi} \cdot \frac{\Delta x}{x^{2}}
$$

and the work $W$ which is done while $q$ is carried from $p$ to infinity is:

which gives:

$$
W=\frac{B Q q}{4 \pi} \int_{x=r}^{x=\infty} \frac{d x}{x^{2}}
$$

$$
W=\frac{B Q q}{4 \pi} \cdot \frac{I}{r}
$$


but this is the amount of work done by the electric field on $q$ coulombs of charge which is carried from $p$ to an infinite distance; therefore, dividing by $q$ we get the work required per coulomb, and this is by definition the potential at $p$. Therefore:

$$
V=\frac{B}{4 \pi} \cdot \frac{Q}{r}
$$

where $V$ is the desired potential at $p$ in volts, the potential being due to the charge of $Q$ coulombs uniformly distributed over the sphere $S$.

Ior. Capacity of a condenser consisting of concentric metal spheres with air between.-Figure II 4 shows a metal sphere of

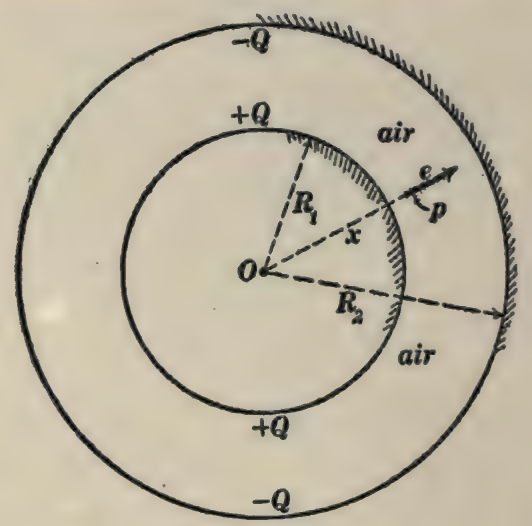

Fig. 114.

radius $R_{1}$ surrounded by a hollow metal sphere of which the inside radius is $R_{2}$. The inner sphere carries a charge of $+Q$ coulombs and the inner surface of the outer sphere carries $-Q$ coulombs; and these charges are uniformly distributed over the respective spherical surfaces because of the complete symmetry. The two spheres constitute a condenser, and therefore:

$$
Q=C E
$$

where $C$ is the capacity of the condenser and $E$ is the electromotive force or potential difference between the spheres. If we 
can calculate the value of $E$ for a given value of $Q$, this equation will give an expression for $C$.

The intensity $e$ of the electric field at the point $p$ in Fig. I I4 is:

$$
e=\frac{B}{4 \pi} \cdot \frac{Q}{x^{2}} \text { volts per centimeter }
$$

(see Art. 92). Multiplying this value of $e$ in volts per centimeter by $\Delta x$ in centimeters we get the electromotive force $\Delta E$ along $\Delta x$. That is:

$$
\Delta E=e \cdot \Delta x=\frac{B Q}{4 \pi} \cdot \frac{\Delta x}{x^{2}}
$$

whence, integrating, we get:

$$
E=\frac{B Q}{4 \pi} \int_{x=R_{1}}^{x=R_{2}} \frac{d x}{x^{2}}=\frac{B Q}{4 \pi}\left(\frac{\mathrm{I}}{R_{1}}-\frac{\mathrm{I}}{R_{2}}\right)
$$

But $C=\frac{Q}{E}$ according to equation (I). Therefore, from equation (4) we get:

$$
C_{\text {farads }}=\frac{4 \pi}{B}\left(\frac{\mathrm{I}}{\frac{\mathrm{I}}{R_{1}}-\frac{\mathrm{I}}{R_{2}}}\right)
$$

where $R_{1}$ and $R_{2}$ are expressed in centimeters, and $B=$ I.I3I $\times \mathrm{IO}^{13}$.

If $R_{2}$ is indefinitely great, then $\frac{I}{R_{2}}$ is negligible as compared with $\frac{\mathrm{I}}{R_{1}}$ and equation (I) becomes:

$$
\left.\begin{array}{l}
\text { Capacity in farads of a sphere at a great } \\
\text { distance from all surrounding bodies. }
\end{array}\right\}=\frac{4 \pi R_{1}}{B}
$$

Note.-Equations (5) and (6) give the values of the capacity when the dielectric is air. For any other dielectric the capacity is $k$ times as great, where $k$ is the inductivity of the dielectric.

I02. Capacity of a condenser consisting of coaxial metal cylinders with air between.-Let Fig. II4 represent a sectional view of two coaxial metal cylinders. The inner cylinder carries 
$+Q$ coulombs per centimeter of length, and the inner surface of the outer cylinder carries $-Q$ coulombs per centimeter of length; and these charges are spread uniformly around the cylinders because of the complete symmetry. The two cylinders constitute a condenser, and therefore:

$$
Q=C E
$$

where $Q$ is the charge per unit length (positive on one cylinder, negative on the other), $C$ is the capacity per unit length, and $E$ is the electromotive force or potential difference between the cylinders. If we can calculate $E$ for a given value of $Q$, this equation will give us an expression for $C$.

The electric field intensity $e$ in volts per centimeter at the point $p$ in Fig. II 4 is:

$$
e=\frac{B Q}{2 \pi} \cdot \frac{\mathbf{I}}{x}
$$

according to Art. 94, where $x$ is the distance of $p$ from the axis $O$ in centimeters. Multiplying the value of $e$ in volts per centimeter by $\Delta x$ centimeters we get the electromotive force along $\Delta x$ in volts, that is, $\Delta E=e \cdot \Delta x$, or:

$$
\Delta E=\frac{B Q}{2 \pi} \cdot \frac{\Delta x}{x}
$$

so that:

$$
E=\frac{B Q}{2 \pi} \int_{x=R_{1}}^{x=R_{a}} \frac{d x}{x}=\frac{B Q}{2 \pi} \log _{\mathrm{e}}\left(\frac{R_{2}}{R_{1}}\right)
$$

But $C=\frac{Q}{E}$ according to equation (I). Therefore, from equation (4), we get:

$$
C=\frac{2 \pi}{B} \cdot \frac{I}{\log _{e}\left(\frac{R_{2}}{R_{1}}\right)}
$$

where $C$ is the capacity in farads of each centimeter of length of the coaxial cylinders, and $B$ is $\mathrm{I}$.I $3 \mathrm{I} \times \mathrm{IO}^{13}$.

Equation (5) refers to coaxial cylinders with air between. 
With any other dielectric the capacity is $k$ times as great where $k$ is the inductivity of the dielectric.

I03. Maximum electrical stress in cable insulation. - Consider a portion $l$ centimeters in length of an electric cable. Let $R_{1}$ centimeters be the radius of the central wire, let $R_{2}$ centimeters be the inside radius of the lead sheath, let $k$ be the inductivity of the dielectric and let $E$ volts be the electromotive force between central wire and sheath. It is desired to find an expression for the electrical stress $e$ in volts per centimeter at the surface of the central wire, this being the place where the electrical stress is a maximum. The capacity of unit length of the cable is given by equation (5) of Art. I02, and the capacity of $l$ centimeters of the cable is $l$ times as great, or $\frac{2 \pi l}{B} \cdot \frac{k}{\log _{\epsilon}\left(\frac{R_{2}}{R_{1}}\right)}$.

Multiplying this by $E$ we get the number of coulombs on $l$ centimeters of the central wire, and multiplying the number of coulombs by $B$ we get the electric flux emanating from the $l$ centimeters of the central wire according to Gauss's theorem (Art. 86). But the electric flux density at the surface of the central wire is $k e$ according to Art. 86, and the area of $l$ centimeters of the central wire is $2 \pi R_{1} l$ so that the electric flux emanating from $l$ centimeters of the central wire is $2 \pi R_{1} l \times k e$. Therefore, placing these two expressions for the electric flux equal to each other we have: -

$$
\text { - } 2 \pi R_{1} l k e=\frac{2 \pi l B E}{B} \cdot \frac{k}{\log _{\epsilon}\left(\frac{R_{2}}{R_{1}}\right)}
$$

from which we get:

$$
e_{\text {at surface of central wire }}=\frac{E}{R_{1}} \cdot \frac{\mathrm{I}}{\log _{e}\left(\frac{R_{2}}{R_{1}}\right)}
$$

I04. Capacity of parallel cylindrical wires in air. - The capacity of a two-wire transmission line in farads per mile of double line is: 


$$
C=\frac{1.943}{10^{8} \times \log _{10}\left(\frac{D-R}{R}\right)}
$$

where $D$ is the distance of the wires apart center to center, and $R$ is the radius of each wire; $D$ and $R$ being both expressed in terms of the same unit. This equation is only approximately true as will appear in the following discussion.*

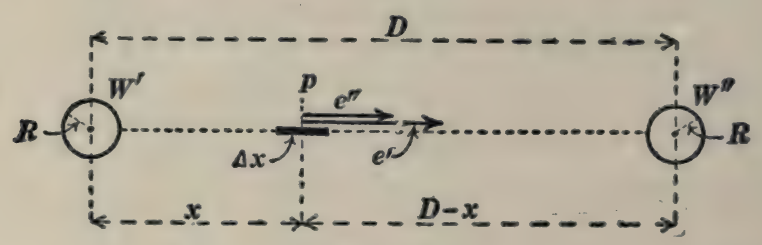

Fig. 115.

The two circles represent sections of the two wires of a transmission line.

Figure II5 is a sectional view of the two wires $W^{\prime} W^{\prime \prime}$ of a transmission line, and to find the capacity per mile it is necessary to calculate the voltage between the wires due to an assumed amount of charge, positive on one wire and negative on the other wire.

The diameter of the wires is small as compared with the distance between them, and therefore each wire may be thought of approximately as a line charge as explained in Art. 94. Therefore according to equation (2) of Art. 94 the electric field intensity in volts per centimeter at the element $\Delta x$ due to the positively charged wire on the left is:

$$
e^{\prime}=\frac{B Q}{2 \pi} \cdot \frac{\mathbf{I}}{x}
$$

and the volts per centimeter at $\Delta x$ due to the negatively charged wire on the right is:

$$
e^{\prime \prime}=\frac{B Q}{2 \pi} \cdot \frac{\mathrm{I}}{D-x}
$$

* A rigorous discussion of the capacity of two parallel metal cylinders is given on pages 273-277 of Franklin's Eleclric Waves, The Macmillan Co., I909. 
where $\pm Q$ is the coulombs per centimeter on the respective wires; $e^{\prime}$ and $e^{\prime \prime}$ are both towards the right as shown in Fig. II 5 and therefore both to be considered as positive.

The total volts per centimerer at $\Delta x$ is $e^{\prime}+e^{\prime \prime}$, and the volts along $\Delta x$ is $\left(e^{\prime}+e^{\prime \prime}\right) \cdot \Delta x$. Therefore:

$$
\Delta E=\frac{B Q}{2 \pi}\left(\frac{\mathrm{I}}{x}+\frac{\mathrm{I}}{D-x}\right) \cdot \Delta x
$$

and by integrating this expression from $x=R$ to $x=D-R$ we get the voltage or potential difference between the wires, namely:

$$
E=\frac{B Q}{\pi} \cdot \log _{e}\left(\frac{D-R}{R}\right)
$$

Now the charge on one mile (I6I,000 centimeters) of the line (positive on one wire and negative on the other) is $16 \mathrm{I}, 000 Q$. Therefore using the value of $Q$ from equation (4) we get:

$$
\text { Charge on one mile of line }=\left[\frac{\mathrm{I} 6 \mathrm{I}, 000 \pi}{B \log _{e}\left(\frac{D-R}{R}\right)}\right] \cdot E
$$

and the expression in the brackets is the capacity in farads of one mile of the double line. To get equation (I) the Naperian logarithm is to be reduced to the common logarithm.

I05. Maximum electric stress between the wires of a transmission line.-The electrical stress at the surface of the wire to the left (or right) in Fig. I 5 may be thought of as being made up of two parts, one part being due to the charge on the wire to the left and the other part being due to the charge on the wire to the right. These two parts are given by equations (I) and (2) of Art. 104 if $R$ be written for $x$. Therefore the total electrical stress at the surface of one of the wires in Fig. II 5 is:

$$
e^{\prime}+e^{\prime \prime}=\frac{B Q}{2 \pi}\left(\frac{\mathrm{I}}{R}+\frac{\mathrm{I}}{D-R}\right)
$$


Therefore, substituting the value of $Q$ from equation (4) of Art. 104, we get:

$\left.\begin{array}{l}\begin{array}{l}\text { Electrical stress at surface of } \\ \text { wires of a transmission line }\end{array}\end{array}\right\}=\frac{E}{2 \log _{e}\left(\frac{D-R}{R}\right)}\left(\frac{\mathrm{I}}{R}+\frac{\mathrm{I}}{D-R}\right)$

This expression gives the electrical stress in volts per centimeter at the surface of either wire in Fig. II5, $E$ being the electromotive force between the wires in volts, $R$ the radius of the wires in centimeters, and $D$ the distance between the wires in centimeters.

I06. Distribution of electric potential in the neighborhood of a line charge.-The electric field at a point distant $r$ centimeters from the axis of a uniformly charged cylinder in air is:

$$
e=\frac{B}{2 \pi} \cdot \frac{Q}{r}
$$

according to equation (2) of Art. 94, and evidently the field does not depend upon the diameter of the cylinder if the charge per unit length $Q$ is given. Therefore we may suppose the charged cylinder to have an indefinitely small diameter, and we thus arrive at the notion of what is called a line charge, namely, a charge of $Q$ coulombs per centimeter distributed uniformly along a straight line. Equation (I) expresses the electric field intensity in the neighborhood of a line charge.

To find the electric potential distribution in the neighborhood of a line charge it is not permissible to choose the infinitely distant region $(r=\infty)$ as the region of zero potential because to do so leads to infinite values of potential everywhere; it is most convenient to choose the region which is at unit distance $(r=\mathrm{I}$ centimeter) from a line charge as the region of zero potential.

The dot $A$ in Fig. II6 is an end view of a line charge of $Q$ coulombs per centimeter, and $O$ is the chosen region of zero potential; and it is desired to find the potential at the point $p$ 
distant $r$ centimeters from $A$. Writing $x$ for $r$ in equation (I), above, we get $\frac{B}{2 \pi} \cdot \frac{Q}{x}$ volts per centimeter as the intensity of the electric field at the element $\Delta x$ in Fig. I I6, and multiplying

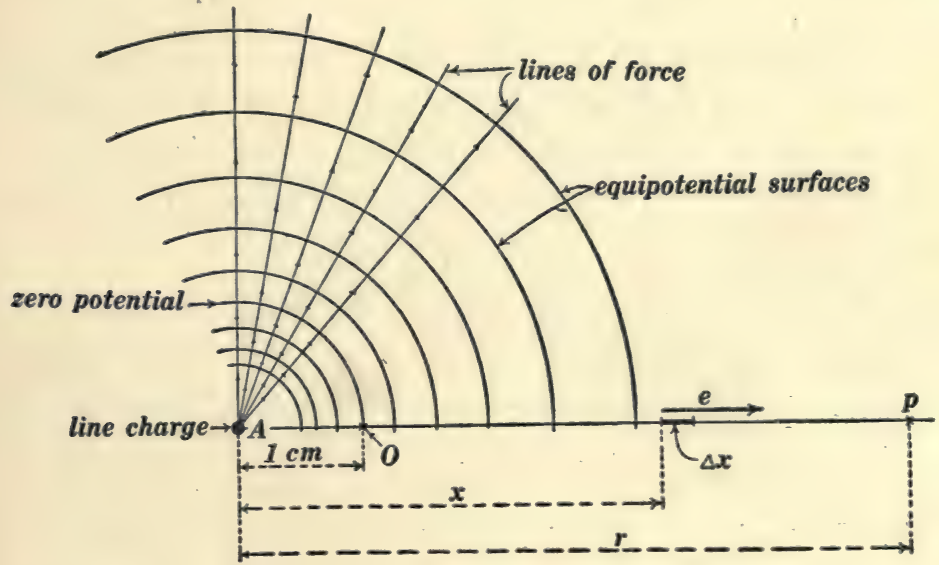

Fig. 116.

this by $\Delta x$ we get the volts along $\Delta x$, namely:

$$
\Delta E=\frac{B Q}{2 \pi} \cdot \frac{\Delta x}{x}
$$

and the total volts between $O$ and $p$ is found by integrating this equation from $x=\mathrm{I}$ to $x=r$ which gives:

$$
E=\frac{B Q}{2 \pi} \cdot \log _{e} r
$$

Now the electric field in Fig. I 16 is directed away from $O$ and towards $p$ all along the path, and therefore work is done by the electric field while a positive charge moves from $O$ to $p$, but the potential at $p$ is defined in Art. 99 as the work done by the electric field while one unit of positive charge is transferred from $p$ to $O$. Therefore the potential at $p$ in Fig. II6 is negative, and from equation (3) we have 


$$
V=-\frac{B Q}{2 \pi} \cdot \log _{e} r
$$

where $V$ is the electric potential in volts at a point $r$ centimeters from a line charge of $Q$ coulombs per centimeter, and $B=$ I.I3I $\times \mathrm{IO}^{13}$.

It is evident that $V$ is a constant for any given value of $r$, and therefore the surface of any circular cylinder with its axis along $A$ is an equi-potential surface. The circles in the upper part of Fig. II6 represent a series of equipotential surfaces.

Distribution of electric potential in the neighborhood of two parallel equal and opposite line charges.-Figure I 7 is a sectional view of two parallel line charges, $+Q$ and $-Q$ coulombs per

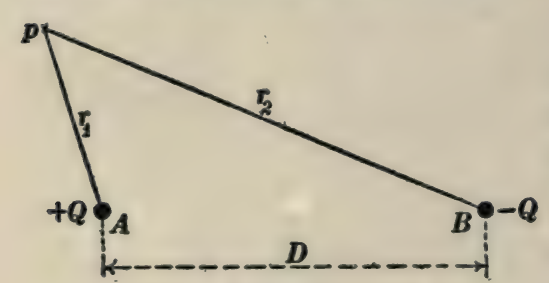

Fig. 117. centimeter respectively. The potential at $p$ due to $+Q$ alone is given by writing $r_{1}$ for $r$ in equation (4), and the potential at $p$ due to $-Q$ alone is given by writing $-Q$ for $Q$ and $r_{2}$ for $r$ in equation (4); and the total potential at $p$ due to both line charges is the algebraic sum of the expressions so found. Therefore:

$$
V=\frac{B Q}{2 \pi} \log _{e}\left(\frac{r_{2}}{r_{1}}\right)
$$

For a series of constant values of $V$ this equation becomes the equation of a series of circles as shown in Fig. II8. These circles represent a series of cylindrical surfaces at right angles to the plane of the paper. These cylindrical surfaces are equipotential surfaces. The lines of force of the electric field due to two parallel equal and opposite line charges are shown in Fig. I19. These lines of force cut the equipotential surfaces at right angles as shown in Fig. II2. 
IDEA OF POTENTIAL.

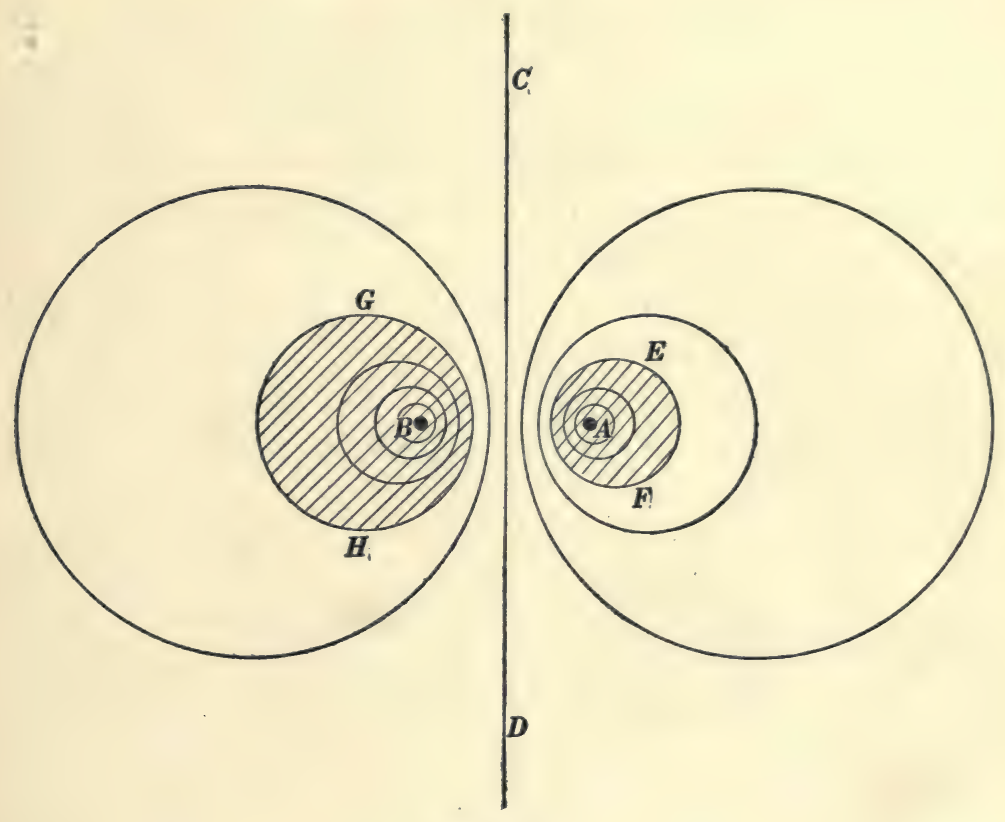

Fig. 118.

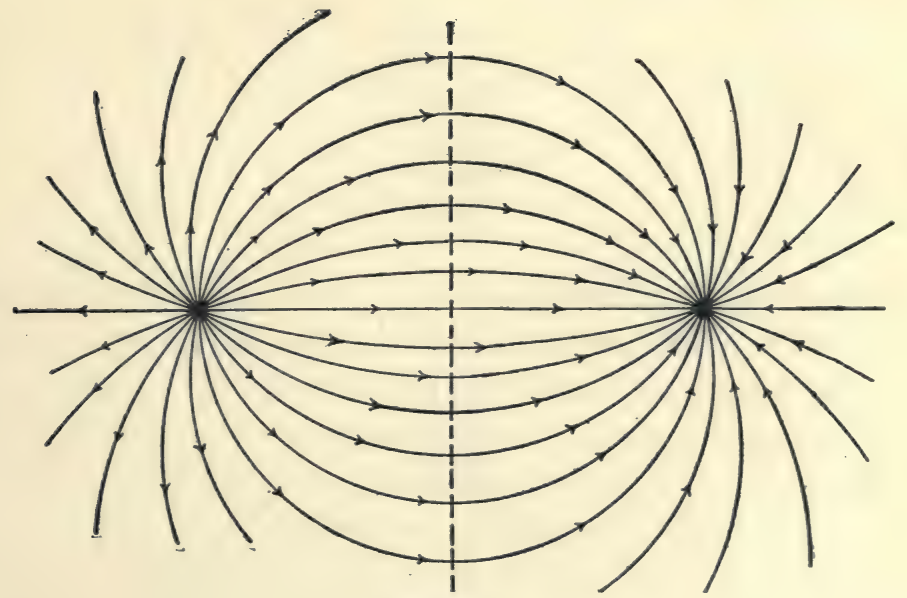

Fig. 119. 
I07. The method of electric images.-Any equipotential surface in an electric field may be wholly or in part replaced by a thin metal shell without altering the electric field distribution; and if an equipotential surface is wholly replaced by a thin metal shell, the electric field on one side of the shell may be obliterated without altering the field on the other side.

Thus one or both of the equipotential surfaces $G H$ and $E F$ in Fig. II 8 may be replaced by metal, and the electric field between them will remain unchanged. In this way it is known that the distribution of electric field between two parallel metal

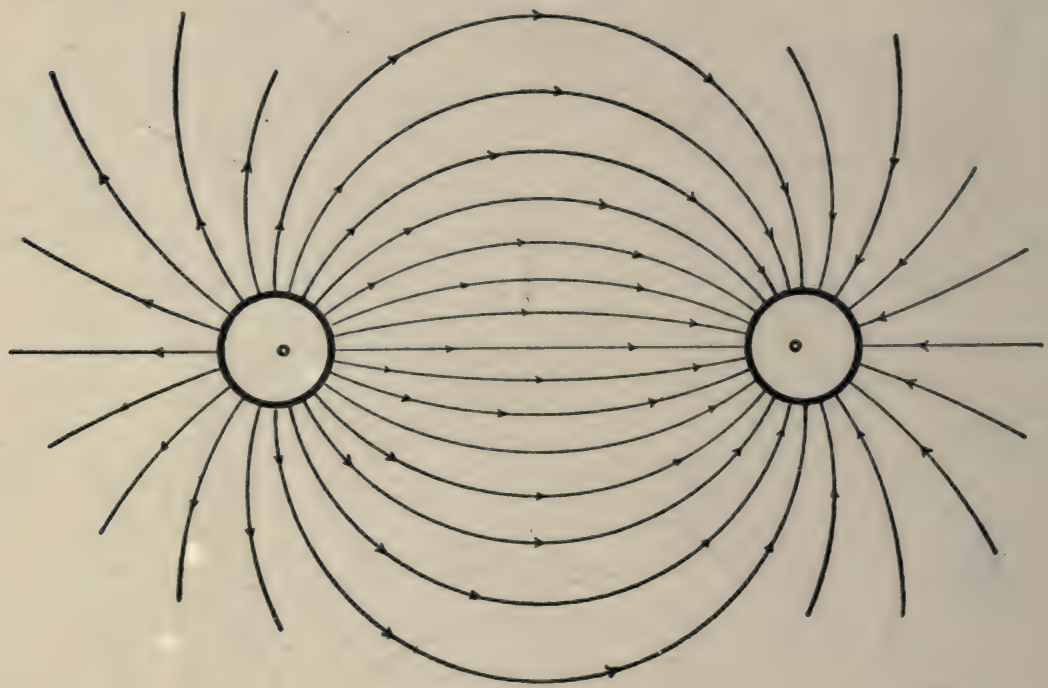

Fig. 120.

cylinders of equal or unequal diameters is identical with a certain part of the electric field between parallel line charges properly spaced. Thus Fig. I20 shows accurately the trend of the electric lines of force between two parallel metal cylinders of the same diameter. The very small circles show the positions of the equivalent line charges. Simi'arly Fig. I2I shows accurately the trend of the lines of force between two parallel metal cylinders one of which surrounds but is not co-axial with the other; the small circles 
$C$ and $C^{\prime}$ show the positions of the equivalent line charges. This method of building up particular cases where the electric field distribution (or potential distribution) is known is called the

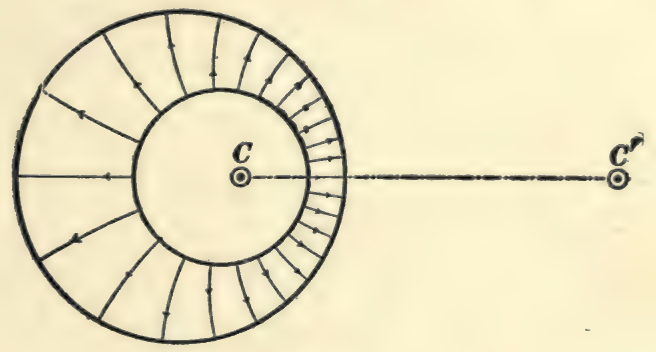

Fig. 121.

method of electrical images. The origin of this term may be illustrated by discussing Fig. I22. A line charge $A$ near and parallel to a metal cylinder $B B$ having an equal and opposite amount of

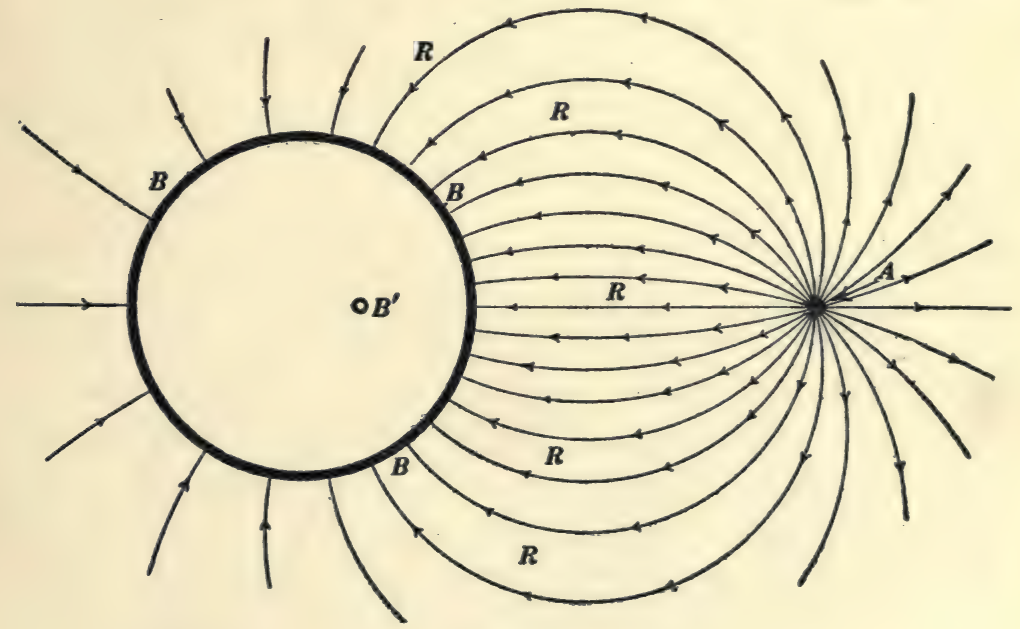

Fig. 122 .

charge per unit length gives in the region $R R R$ an electric field distribution which is exactly the same as would be produced by $A$ and an equal and opposite line charge at $B^{\prime}$ with the metal cylinder removed. Therefore one may think of the field $R R R$ as being 
produced by $A$ and a fictitious line charge at $B^{\prime}$ which is called the electric image of $A$ with respect to the metal cylinder $B B$.

ro8. Another important example of the method of images. A uniform electric field as modified by a cylindrical conductor.Figure 123 shows the trend of the lines of force in the neighbor-

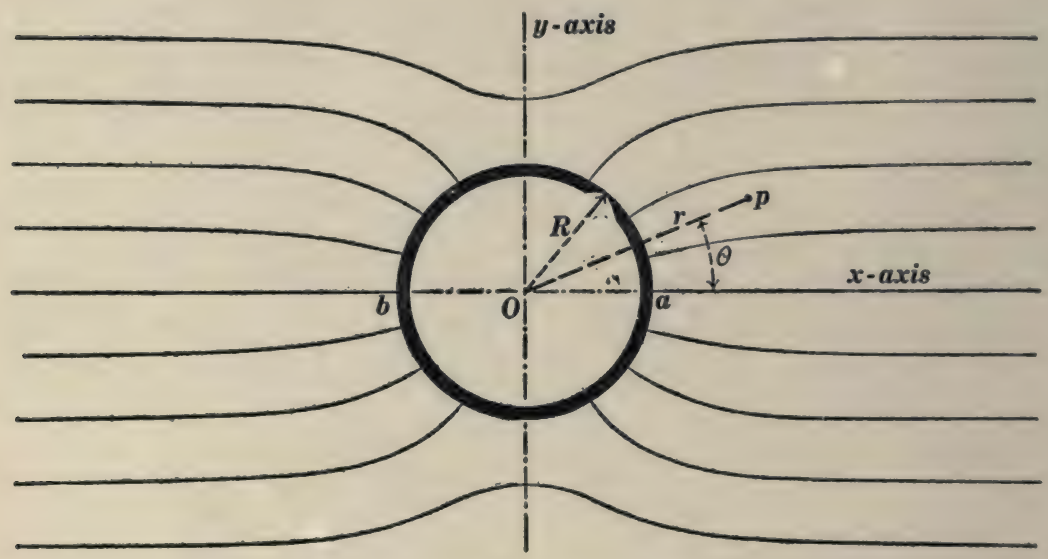

Fig. 123.

hood of a metal cylinder of radius $R$ in a region which would be a uniform electric field parallel to the $x$-axis if it were not for the presence of the metal cylinder. The mathematical formulation of the field distribution in any case is greatly simplified by using the idea of potential. We will proceed therefore to find an expression for the potential at any point $p$ in Fig. I23 of which the polar coördinates with respect to $O$ are $r$ and $\theta, \theta$ being the angle between $r$ and the $x$-axis of reference.

The finding of this mathematical expression depends upon the combining of two potentials, namely, (a) the potential due to the original undisturbed uniform field $e$ parallel to the $x$-axis, and (b) the potential due to what is called a line doublet.

Potential due to a uniform field of intensity $e$ parallel to the $x$-axis.-Let $V^{\prime}$. be the potential which is associated with $e$; 
then $\frac{d V^{\prime}}{d x}=e$, so that $V^{\prime}=e x+$ a constant. Therefore if we choose the $y$-axis as the region of zero potential we must have $V^{\prime}=0$ when $x=0$. Therefore the constant of integration is zero, and we have:

$$
V^{\prime}=e x
$$

where $V^{\prime}$ is the potential at any point whose abscissa is $x$.

Potential due to a line doublet.-Consider two equal and opposite line charges parallel to each other and at a distance $\Delta x$ apart, let $+Q$ and $-Q$ be the charges per centimeter, and let the product $Q \cdot \Delta x$ be a finite quantity $M$. Such a pair of infinitesimally distant, equal and opposite line charges is called a line doublet, and $M$ is called the moment of the doublet.

The potential at a point due to a line doublet is found as follows: The potential at the point $p$ in Fig. I24 due to the line

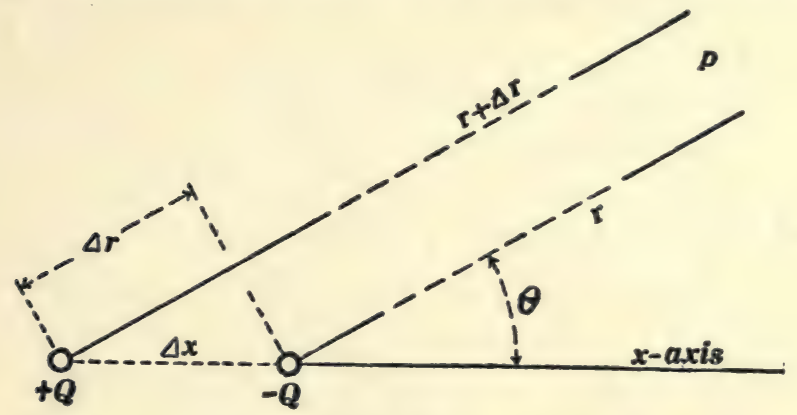

Fig. 124.

The distance $r$ is supposed to be very large in comparison with $\Delta x$.

charge $+Q$ is $-\frac{B Q}{2 \pi} \cdot \log _{e} r$, and the potential at $p$ due to the line charge $-Q$ is $+\frac{B Q}{2 \pi} \cdot \log _{e}(r+\Delta r)$, according to equation (4) of Art. Io6. Therefore the net potential at $p$ due to both line charges is:

$$
\frac{B Q}{2 \pi}\left[\log _{e}(r+\Delta r)-\log _{e} r\right]=\frac{B Q}{2 \pi} \cdot d\left(\log _{e} r\right)=\frac{B Q}{2 \pi} \cdot \frac{\mathbf{I}}{r} \cdot d r
$$


but $\Delta r=\Delta x \cdot \cos \theta$ (see Fig. 124), and $M=Q \cdot \Delta x$; therefore this expression for the potential at $p$ in Fig. I24 reduces to:

$$
V^{\prime \prime}=\frac{B}{2 \pi} \cdot M \frac{\cos \theta}{r}
$$

If the positive and negative line charges are interchanged in Fig. I24 the algebraic sign of the potential $V^{\prime \prime}$. will be changed and we will have:

$$
V^{\prime \prime}=-\frac{B}{2 \pi} \cdot M \frac{\cos \theta}{r}
$$

These expressions [equations (2) and (3)] for the potential of a line doublet give $V^{\prime \prime}=0$ when $\theta=90^{\circ}$ if $r$ is not zero. That is, the potential along the $y$-axis is zero except at the origin $O$ in Fig. I23 when a horizontal doublet is placed at $O$.

The potential in a region where a line doublet is placed and which but for the presence of the doublet would be a uniform electric field at right angles to the doublet.-This potential is found by adding the above potentials as expressed by equations (I) and (2), or (I) and (3); and for $x$ in equation (I) we may write $r \cos \theta$. Thus we get for the combined potential

$$
V=\left(e r \pm \frac{B M}{2 \pi r}\right) \cos \theta
$$

If the negative sign is chosen (which means that the line doublet is the reverse of what is shown in Fig. 124), then for a certain value of $r$ the quantity in the parenthesis will be zero. Therefore $V$ will be zero for this value of $r$ whatever the value of $\theta$ may be. That is to say one of the constant potential surfaces surrounding the line doublet placed in a uniform field will be the surface of a circular cylinder, and this particular cylindrical surface may be replaced by a metal cylinder without altering the field distribution. Therefore

$$
V=\left(e r-\frac{B M}{2 \pi r}\right) \cos \theta
$$


is the potential at any point $[r, \theta]$ outside of a metal cylinder placed in a region which but for the presence of the cylinder would be a uniform field of intensity $e$ at right angles to the axis of the cylinder. The moment $M$ of the line doublet must be such as to give a value of zero to the quantity in the parenthesis when the radius of the cylinder $R$ is substituted for $r$. That is, we must have:

$$
e R-\frac{B M}{2 \pi R}=0
$$

or

$$
M=\frac{2 \pi}{B} \cdot e R^{2}
$$

Substituting the value of $M$ from (6) in (5), we get:

$$
V=\left(e r-\frac{e R^{2}}{r}\right) \cos \theta
$$

which expresses the potential at each point $(r, \theta)$ near a cylinder of radius $R$ placed in a region which but for the presence of the cylinder would be a uniform electric field of intensity $e$ at right angles to the axis of the cylinder, as shown in Fig. 123. The line doublet is the image in the metal cylinder of the distant charges which produce the uniform field.

It is evident from Fig. I23 that the presence of the metal cylinder causes a concentration of electrical stress, and the maximum stress is at $a$ (or $b$ ) where $r=R$ and $\theta=0$. But the electric field at any point is the potential gradient at that point. Therefore the field intensity at $a$, being evidently parallel to the $x$-axis, is found by differentiating $V$ in equation (7) with respect to $r$ and placing $\theta=0$ and $r=R$ in the result, because $r$ is parallel to $x$ when $\theta$ is zero. In this way we get:

$$
\left\{\begin{array}{c}
\text { field intensity at } \\
a \text { in Fig. } 123
\end{array}\right\}=2 e
$$

That is, the field intensity at $a$ in Fig. I23 is twice as great as the intensity $e$ of the original uniform field. 
109. The production of any desired field distribution in a region by the creation of proper potential values everywhere over the boundary of the region.-The distribution of electric field in a region depends upon two things; namely, (a) Upon the distribution of electric charge over the boundary of the region, and $(b)$ upon the distribution of electric charge throughout the region itself. When there is no charge in the region, the boundary distribution alone determines the field throughout the region. In practice it is much easier to produce assigned potential values than to produce assigned distributions of charge. Therefore for practical purposes the following proposition may be used instead of the above. When there are no free electric charges in a region, the potential values over the boundary of the region completely determine the field distribution in the region.

Example 1.-Figure 125 represents the lines of force surrounding a line charge, the line charge being perpendicular to

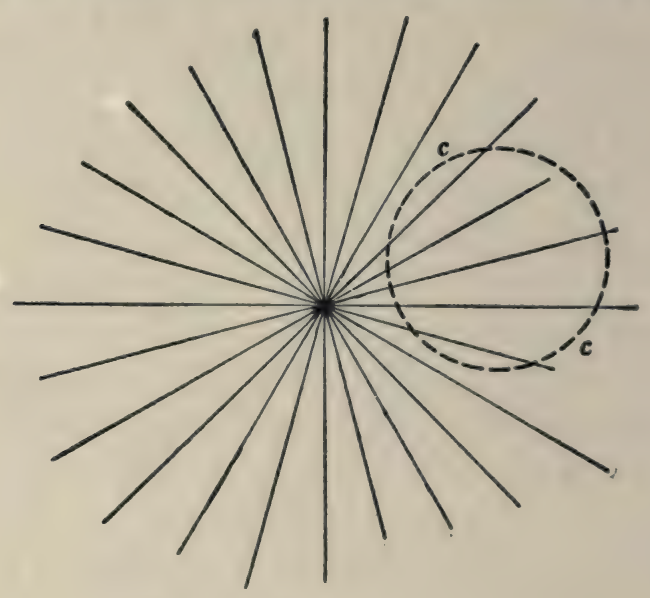

Fig. 125.

the plane of the paper. The dotted circle in Fig. 125 represents a cylinder with its axis parallel to the line charge. Imagine this cylinder to be made of narrow insulated strips of metal as represented by the separate dots of the circumference, and imagine 
earh strip to be brought by proper means to a potential equal to the potential at the strip due to the line charge in Fig. 125. We would thus have the potential fixed or determined at every point on the surface of the dotted cylinder, and the electric field distribution inside of the cylinder would be as shown in Fig. I26. That is, the electric field inside of the cylinder $c c$ would be exactly like the field due to the line charge in Fig. 125,

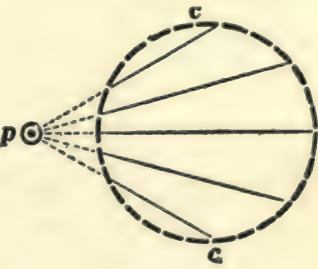

Fig. 126. and this would be the case whatever the state of affairs outside of the cylinder $c c$ might be.

Example 2.-A flat metal sheet $A A$, Fig. 127, is at zero potential, and a series of insulated metal strips $B B$ very close together and perpendicular to the plane of the paper are brought by proper

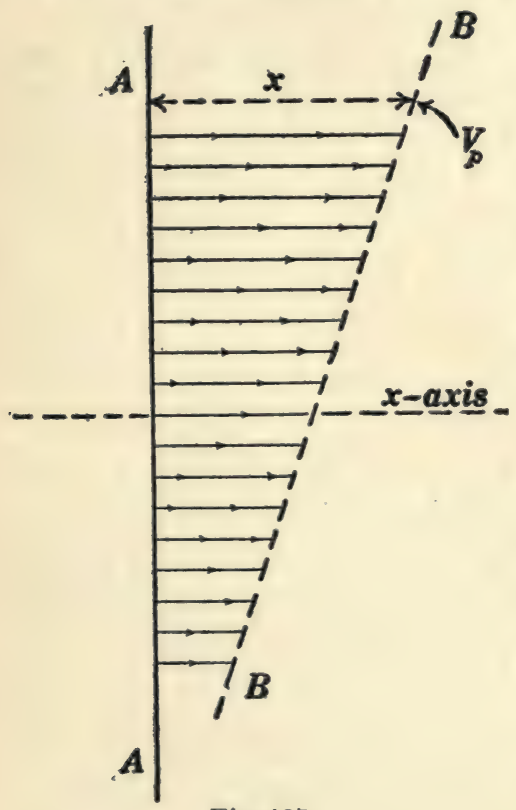

Fig. 127. means to potential values such that the potential $V_{p}$ of any strip is proportional to its distance $x$ from $A A$. Under these conditions the region between $A A$ and $B B$ will be a uniform electric field as indicated by the lines of force in Fig. I27.

Fortescue's method for designing insulating structures. - The method illustrated in Figs. I25 to 127 for producing any assigned electric field distribution in a given region has been proposed by Mr. Charles L. Fortescue as a basis for the design of insulating structures. The essential features of $\mathrm{Mr}$. Fortescue's method may be brought out by the following prob- 
lem: It is required to produce a uniform electric field parallel to the $y$-axis in the region of the coarse lines of force in Fig. 128 , and a cylindrically radiating electric field in the region of the fine lines of force in Fig. 128; and to provide for the proper bounding of

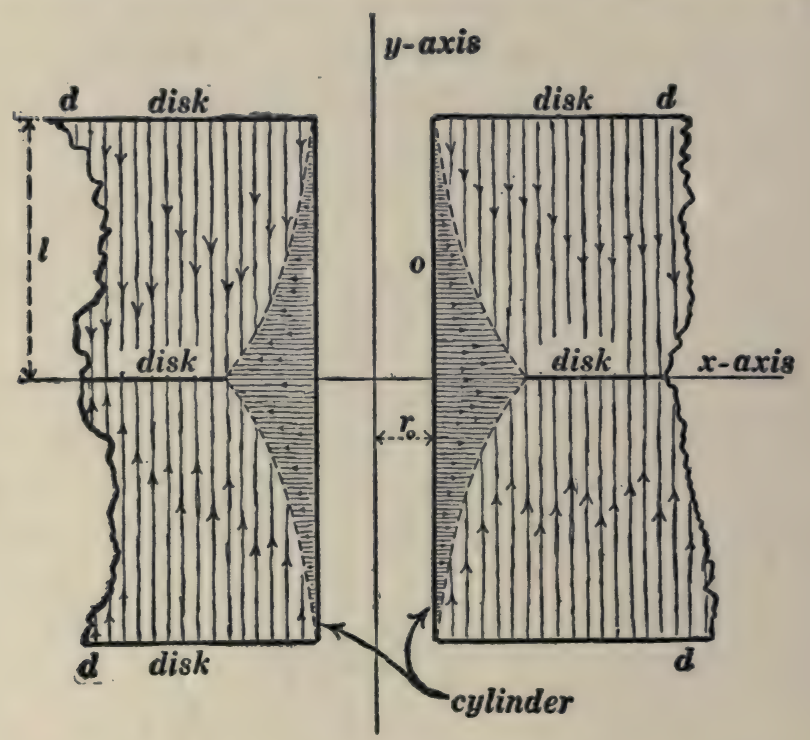

Fig. 128.

these two regions. By cylindrically radiating field is here meant a field like that which would radiate from a line charge lying along the $y$-axis in Fig. 128.

Let us assume that the metal cylinder of radius $r_{0}$ and the metal disks $d d$ are at zero potential, and let us consider the potential at any point $p$ of which the coördinates are $x$ and $y$. The potential at $p$ due to the radiating field, on the assumption that the radial field reaches to $p$; would be:

$$
k \log _{e} x-k \log _{e} r_{0}
$$

where $k$ is a constant; and the potential at $p$ due to the uniform field would be:

$$
e(l-y)
$$


where $e$ is the intensity of the uniform field. The locus of the points where the two fields would give the same potential is shown by the dotted curve in Fig. 128, and the equation to this curve may be found by equating the two expressions for potential given above.

In order that the two fields as indicated by the coarse and fine lines of force in Fig. I28 may actually be obtained, it is sufficient to have the cylinder of radius $r_{o}$ and the end disks at zero potential; to have the middle disk at potential $\mathrm{el}$; and to pro-

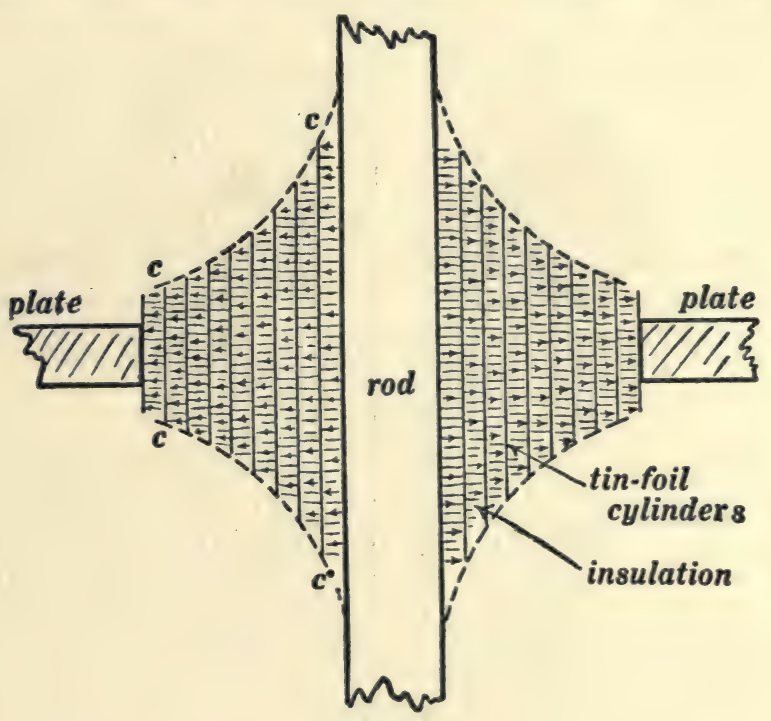

Fig. 129.

vide a number of insulated rings of tinfoil along the common boundary of the two fields (as represented in section by the separate dots of the dotted curve), these tinfoil rings being brought to potentials which conform to both fields. The arrangement shown in Fig. I 28 is such as would be used around one leg of the core of a core-type transformer, and the various parts of the boundary (tinfoil rings, disks, etc.) are brought to the desired potentials by being connected to tap-points on the high-voltage coil of the transformer. 
IIo. The condenser type of insulating bushing again considered.-The radial electric field surrounding a line charge decreases in intensity at increasing distances from the line charge, and to produce a radial field of constant intensity in a homogeneous dielectric free charge would have to be distributed in a certain definite manner throughout the dielectric. This condition is approximately realized in the condenser type of insulating bushing as described in Art. 89, the free charge being on the tin-

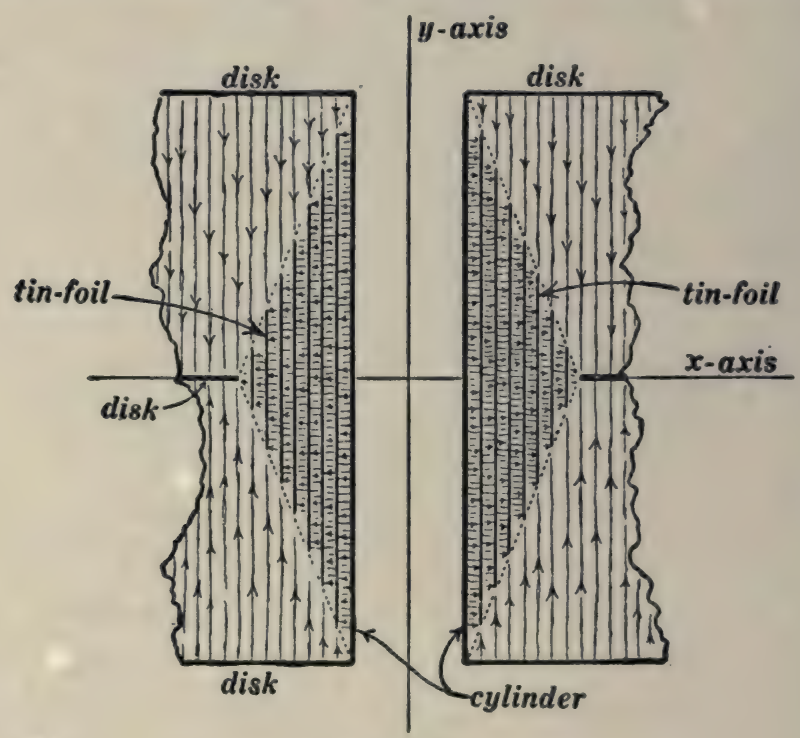

Fig. 130 .

foil sheets. A sectional view of such a bushing is shown in Fig. 129. The theoretically correct shape of the curve $c c$ in Fig. I29 is an equilateral hyperbola, because the successive tinfoil cylinders must have the same area, as explained in Art. 89.

No attempt is made to show the lines of force in the region surrounding the bushing in Fig. 129. As a matter of fact this electric field is greatly concentrated in the region immediately surrounding the rod at the ends of the bushing. This concentration of electrical stress is obviated by Mr. Fortescue's method as follows: 
III. Fortescue's design again.-There is a constant potential difference between successive tinfoil cylinders in Fig. I29, and a constant potential difference between successive tinfoil cylinders is to be provided for in Fig. I30 as explained later; but if the potentials of the edges of the tinfoil cylinders in Fig. I30 are to be conformable to the uniform electric field which is represented by the coarse lines of force in Fig. I30 it is necessary to terminate the cylinders along the dotted straight lines as shown. With this arrangement, however, the tinfoil cylinders do not all have the same area and they do not automatically adjust themselves to equal potential differences as explained in Art. 89. Therefore to provide for equal potential differences between successive tinfoil cylinders in Fig. I3O it is necessary to connect the successive cylinders to proper points in the winding of the transformer (the bushing in Figs. I29 and I30 being used to insulate the wires which pass through a metal case to the highvoltage windings of a transformer).

\section{PROBLEMS.}

I. All space is filled with a fluid moving parallel to the $x$-axis of reference at a uniform velocity of ro centimeters per second. Find an expression for the velocity potential of the fluid. Ans. Iox + any constant.

2. Derive an expression for the electric potential of a uniform electric field which is parallel to the $x$-axis of reference and of which the intensity is $\mathrm{I}, 000$ volts per centimeter. Ans. I,000x + any constant.

3. The entire region throughout a room is a uniform electric field directed vertically upwards and its intensity is 2,000 volts per centimeter. (a) Choosing the floor as the region of zero potential, what is the potential at a point $\mathbf{I} 50$ centimeters above the floor? (b) What kind of lines are the lines of force, straight or curved, and in what direction? (c) What kind of surfaces are the surfaces of equipotential, plane or curved, and in what direction do these surfaces lie? Ans. (a) 300,000 volts. 
Straight lines, perpendicular to floor. (c) Plane surfaces, parallel to floor.

4. Given two parallel metal plates i5 centimeters apart to which a 10,000-volt battery is connected. Imagine a line $X$ drawn straight from plate to plate. Choose the negatively charged plate as the region of zero potential; and plot a curve of which the abscissas are distances measured along the line $X$ and of which the ordinates represent the values of the potential at points along this line.

5. An inclined plane is viewed from above. A series of contour lines and of slope lines are drawn upon the plane. Make a diagram showing the appearance of these lines as projected upon the base plane.

6. A circular cone is viewed from above and a series of contour lines and slope lines are seen projected upon the base plane of the cone. Draw a diagram showing the appearance of these lines on the base plane.

7. A sphere of 100 centimeters radius has upon it a charge of $10^{-6}$ coulombs, and the sphere stands in air at a very great distance from all surrounding bodies. Taking the infinitely distant region as the region of zero potential, find the potential of the sphere. Ans. 8,600 volts.

8. Find the maximum electrical stress in volts per centimeter in the insulating material of a cable of which the wire core is cylindrical and 0.5 centimeter in diameter, the inside diameter of the lead sheath being 3 centimeters and the electromotive force between sheath and core being 20,000 volts. Ans. 44,540 volts per centimeter.

9. A transmission line consists of two No. I B. \& S. copper wires at a distance of 2 feet apart center to center. Find the capacity of 20 miles of this line. Ans. 0.175 microfarad.

I0. With an electromotive force of 50,000 volts between the wires of the previous problem find the maximum electrical stress in the air. Ans. 34,070 volts per inch. 


\section{CHAPTER IX.}

\section{ELECTRIC OSCILLATIONS AND ELECTRIC WAVES.}

\section{II2. Mechanical and electrical parallels.-A great help to the}

clear understanding of the elementary theory of electricity and magnetism is afforded by the identity of many of the equations of mechanics and electricity as exhibited in the following schedule.

Translatory Motion

$$
x=v t
$$

in which $x$ is the distance traveled in $t$ seconds by a body moving at velocity $v$.

$$
W=F x
$$

in which $W$ is the work done by a force $F$ while the body upon which the force acts moves a distance $x$ in the direction of the force.

$$
P=F \vartheta
$$

in which $P$ is the power developed by a force $F$ acting upon a body moving at velocity $v$ in the direction of $F$.

$$
W=\frac{1}{2} m v^{2}
$$

in which $W$ is the kinetic energy of a mass $m$ moving at velocity $v$.

$$
F=m \frac{d v}{d t}
$$

in which $F$ is the unbalanced force required to

\section{Rotatory Motion}

$$
\phi=\omega t
$$

in which $\phi$ is the angle turned in $t$ seconds by a body turning at angular velocity $\omega$.

$$
W=T \phi
$$

in which $W$ is the work done by a torque $T$ while the body upon which the torque acts turns through an angle $\phi$ about the axis of the torque.

$$
P=T \omega
$$

in which $P$ is the power developed by a torque $T$ acting on a body turning at angular velocity $\omega$ about the axis of $T$.

$$
W=\frac{1}{2} K \omega^{2}
$$

in which $W$ is the kinetic energy of a wheel of moment of inertia $K$ turning at angular velocity $\omega$.

$$
T=K \frac{d \omega}{d t}
$$

in which $T$ is the unbalanced torque required to
Electricity and Magnetism

$$
q=i t
$$

in which $q$ is the electric charge which in $t$ seconds flows through a circuit carrying a current $i$.

$$
W=E q
$$

in which $W$ is the work done by an electromotive force $E$ in pushing a charge $q$ through a circuit.

$$
P=E i
$$

in which $P$ is the power developed by an electromotive force $E$ in pushing a current $i$ through a circuit.

$$
W=\frac{1}{2} L i^{2}
$$

in which $W$ is the kinetic energy of a coil of inductance $L$ carrying a current $i$.

$$
E=L \frac{d i}{d t}
$$

in which $E$ is the "unbalanced" electromotive force 
cause the velocity of a body of mass $m$ to increase at the rate $\frac{d v}{d t}$.

$$
\begin{gathered}
x=a F \\
\frac{4 \pi^{2} m}{\tau^{2}}=\frac{I}{a}
\end{gathered}
$$

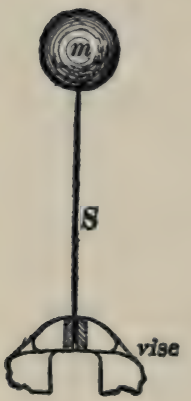

Fig. 131. cause the angular velocity $\mid$ required to cause a curof a wheel of moment of rent in a coil of inductance inertia $K$ to increase at $L$ to increase at the rate the rate $\frac{d \omega}{d t}$.

$$
\begin{gathered}
\phi=b T \\
\frac{4 \pi^{2} K}{\tau^{2}}=\frac{I}{b}
\end{gathered}
$$

$$
\begin{array}{cc}
\frac{d i}{d i} . & \\
& q=C E \\
& \frac{4 \pi^{2} L}{\tau^{2}}=\frac{I}{C}
\end{array}
$$
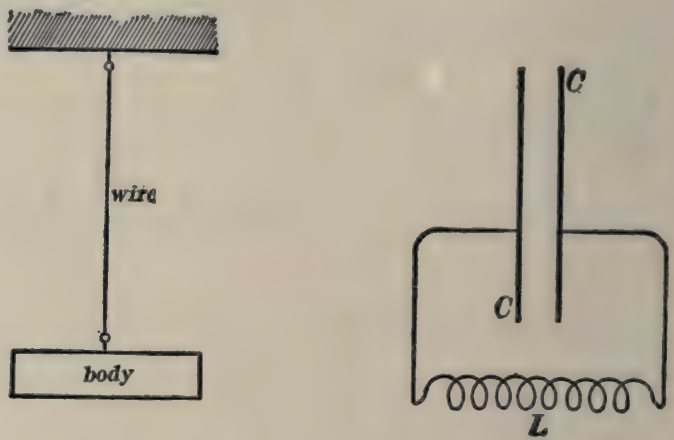

Fig. 132.

Fig. 133.
A body of mass $m$ is supported by a flat spring $S$, clamped in a vise as shown in Fig. I3r. A force $F$ pushing sidewise on $m$ moves it a distance $\boldsymbol{x}$, which is proportional to $F$, according to equation (I6). When started, the body $m$ will continue to vibrate back and forth and the period $\tau$ of its vibrations is determined by equation (I9).
A body of moment of inertia $K$ is hung by a wire as shown in Fig 132 . A torque $T$ acting on the body will turn the body and twist the wire through an angle $\phi$, which is proportional to $T$, according to equation (I7). When started, the body will vibrate about the wire as an axis and the period $\tau$ of its vibrations is determined by equation (20).
A condenser $C$ is connected to the terminals of a coil of inductance $L$ as shown in Fig. 133. An electromotive force $E$ acting anywhere in the circuit pushes into the condenser a charge $q$, which is proportional to $E$, according to equation (I8). When started, the electric charge will surge back and forth through the coil, constituting what is called an oscillatory current and the period of one oscillation is determined by equation (2I).

Note.-Frictional opposition to the motion in Figs. I3I and I32, and the resistance of the circuit in Fig. I33 are supposed to be zero. 
II3. The electric oscillator.-The arrangement shown in Fig. I33 of Art. II2, consisting of an inductance $L$ and a condenser $C$ connected as shown, is called an electric oscillator. This type of oscillator does not radiate energy in the form of electric waves to any appreciable extent, whereas the open oscillator which is shown in Fig. I34 does radiate energy.

Equation (2I) of Art. II2 is true only when the resistance of the circuit in Fig. I33 is negligibly small and when no appreciable energy is radiated in the form of electric

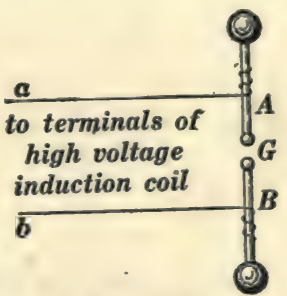

Fig. 134. waves. Under these conditions the differential equation* of "motion" of the arrangement in Fig. I33 is:

$$
L \frac{d^{2} q}{d t^{2}}+\frac{q}{C}=0
$$

The complete solution $\dagger$ of this equation is:

$$
q=Q \sin (\omega t-\theta)
$$

where $Q$ and $\theta$ are constants of integration and

$$
\omega=\frac{\mathbf{I}}{\sqrt{L C}}
$$

The period or time of one complete oscillation is the time required for the angle $\omega t$ to increase from zero to $2 \pi$. That is $\omega \tau=2 \pi$, so that:

$$
\tau=2 \pi \sqrt{L C}
$$

which is the same as equation (2I) of Art. II2.

When the circuit in Fig. I33 of Art. II 2 has an appreciable resistance, the differential equation of its "motion" is:

$$
C L \frac{d^{2} q}{d t^{2}}+C R \frac{d q}{d t}+q=0
$$

* See derivation of equation (4) below.

† See Franklin, MacNutt and Charles's Calculus, pages $18 \mathrm{I}-185$, published by the authors, South Bethlehem, Pa., 1913. 
and the general solution of this equation is:

$$
q=Q \epsilon^{-\alpha t} \sin (\omega t-\theta)
$$

where $Q$ and $\theta$ are constants of integration, and $\alpha$ and $\omega$ are:

and

$$
\alpha=\frac{R}{2 L}
$$

$$
\omega=\sqrt{\frac{\mathrm{I}}{L C}-\frac{R^{2}}{4 L^{2}}}
$$

Derivation of equation (4).- - Let $q$ be the charge on the condenser $C$ in Fig. I33 of Art. II2 at a given instant. Then $\frac{d q}{d t}$ is the current in the circuit, and $\frac{d^{2} q}{d t^{2}}$ is the rate of increase of the current. The electromotive force which acts on the circuit is used (a) in part to hold the charge $q$ on the condenser (the part so used is equal to $\left.\frac{q}{C}\right),(b)$ in part to overcome the resistance of the circuit (the part so used is equal to $R i$ or $R \frac{d q}{d t}$ ), and (c) in part to make the current increase (the part so used is equal to $L \frac{d i}{d t}$ or $\left.L \frac{d^{2} q}{d t^{2}}\right)$. But when the system shown in Fig. I33 is oscillating freely there is no electromotive force acting upon it from outside and therefore the sum of the three parts $(a),(b)$ and $(c)$ is zero.

THE DIFFERENTIAL EQUATION OF WAVE MOTION.

II4. The equation of a traveling curve.-Let $c c$, Fig. I35, be a curve which is stationary with respect to the origin $O^{\prime}$, the equation of the curve with respect to the origin $O^{\prime}$ is:

$$
y=F\left(x^{\prime}\right)
$$

Suppose, however, that the curve $c c$ and the origin $O^{\prime}$ are both traveling to the right at velocity $v$ so that the abscissa of the moving origin $O^{\prime}$ with respect to the stationary origin $O$ is $v t$ as shown. Then the abscissa $x$ of any point on the curve $c c$ 
is $x=x^{\prime}+v t$ so that $x^{\prime}=x-v t$. Therefore, substituting this value of $x^{\prime}$ in equation (I) we have:

$$
y=F(x-v t)
$$

and this is the equation of the traveling curve $c c$ referred to the stationary origin $O$.

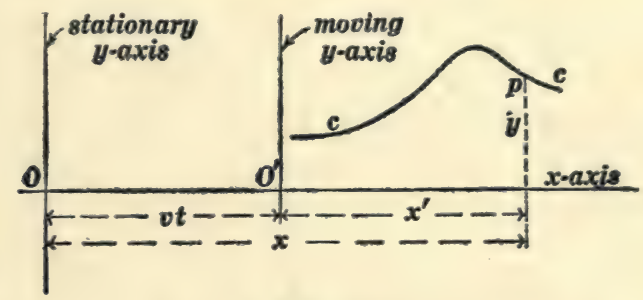

Fig. 135.

Similarly it may be shown that

$$
y=f(x+v t)
$$

is the equation of a curve traveling to the left at velocity $v$ with respect to a stationary origin.

The two equations (2) and (3) satisfy one of the most important of the differential equations of physics, namely, the differential equation of wave motion for the case in which no energy is dissipated by friction or resistance; and it is helpful to derive this differential equation from equation (2) or (3) as follows:

Let the quantity $x-v t$ be represented by the single letter $z$. That is:

and equation (2) becomes:

$$
z=x-v t
$$

$$
y=F(z)
$$

Let $\frac{d y}{d z}$ be represented by $F^{\prime}(z)$ and let $\frac{d^{2} y}{d z^{2}}$ be represented by $F^{\prime \prime}(z)$. Then, according to the rule for differentiating a function of a function, we have:

$$
\frac{\partial y}{\partial x}=\frac{d y}{d z} \cdot \frac{\partial z}{\partial x}
$$

but $\frac{\partial z}{\partial x}=\mathbf{I}$, so that 


$$
\frac{\partial y}{\partial x}=F^{\prime}(z)
$$

Differentiating again, we get:

$$
\frac{\partial^{2} y}{\partial x^{2}}=\frac{d\left[F^{\prime}(z)\right]}{d z} \cdot \frac{\partial z}{\partial x}
$$

but $\frac{\partial z}{\partial x}=\mathrm{I}$ and $\frac{d\left[F^{\prime}(z)\right]}{d z}=F^{\prime \prime}(z)$, therefore, we have:

$$
\frac{\partial^{2} y}{\partial x^{2}}=F^{\prime \prime}(z)
$$

By differentiating twice with respect to $t$ we get:

$$
\frac{\partial^{2} y}{d t^{2}}=v^{2} F^{\prime \prime}(z)
$$

and by combining equations (9) and (Io) we get:

$$
\frac{\partial^{2} y}{\partial t^{2}}=v^{2} \frac{\partial^{2} y}{\partial x^{2}}
$$

This same result would be obtained from equation (3) by proceeding as above. Indeed equations (2) and (3) both satisfy the differential equation (II) and the general solution of (II) is:

$$
y=F(x-v t)+f(x+v t)
$$

This equation represents two curves of any shape superposed upon each other, one traveling to the right at velocity $v$, the other traveling to the left at velocity $v$.

II5. Equation of motion of a stretched string.-It is very helpful to consider wave motion on a stretched string before considering the equations of electric wave motion on a transmission line. When a stretched string is in equilibrium it is, of course, straight. Let us choose this equilibrium position of the string as the $x$-axis of reference as shown in Figs. I 36 and 137 , and let us set up the differential equation which expresses the mode of motion of the string while the string is vibrating or while a bend is traveling along the string as a wave. We will assume that each part of the string moves only up and down (at right 
angles to the $x$-axis in Figs. 136 and 137), and we will assume that the string is perfectly flexible.* Under these conditions the $x$-component of the tension of the string is always and everywhere equal to a constant $T$.

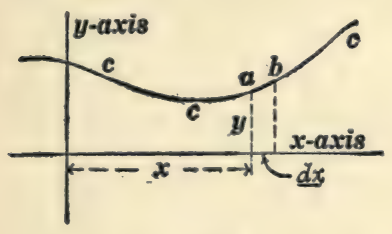

Fig. 136.

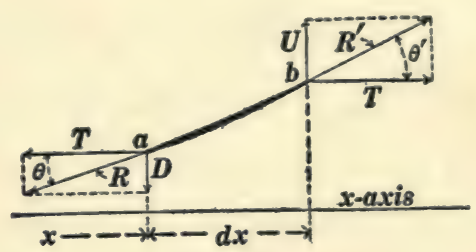

Fig. 137.

Let the curve $c c c$, Fig. 136 , be the configuration of the string at a given instant $t$, that is, $c c c$ is what a photographer would call a snapshot of the moving string. The shape of the curve ccc defines the ordinate $y$ as a function of $x$, and the steepness of the curve $c c c$ at a point is the value of $\frac{d y}{d x}$ at that point.

Consider the very short portion $a b$ of the string. The length of this portion of the string when the string lies along the $x$-axis (in equilibrium) is $d x$, and the mass of the portion is $m \cdot d x$ grams, where $m$ is the mass of one centimeter of the string in grams. An enlarged view of the very short portion of the string is shown in Fig. I37. The adjacent parts of the string pull on the portion $a b$; and the forces $R$ and $R^{\prime}$ thus exerted on $a b$ are parallel to the string at $a$ and at $b$ respectively. The $x$ component of $R$ is the force $T$ to the left, and the $x$-component of $R^{\prime}$ is the force $T$ to the right. Therefore the downward force $D$ is equal to $T \tan \theta$, and the upward force $U$ is equal to $T \tan \theta^{\prime}$; and the net upward force $U-D$ which acts on the portion $a b$ of the string is equal to $T\left(\tan \theta^{\prime}-\tan \theta\right)$. But $\tan \theta$ is equal to the value of $\frac{d y}{d x}$ at $a$ and $\tan \theta^{\prime}$ is equal to the value of $\frac{d y}{d x}$ at $b$. Therefore the difference, $\tan \theta^{\prime}-\tan \theta$,

\footnotetext{
* This means that the only thing that keeps the string straight is its tension.
} 
is the increase of $\frac{d y}{d x}$ from $a$ to $b$, and this increase is equal to $\frac{d^{2} y}{d x^{2}} \cdot d x$. This is evident when we consider that $\frac{d^{2} y}{d x^{2}}$ is the rate of increase of $\frac{d y}{d x}$ with respect to $x$. Therefore, substituting $\frac{d^{2} y}{d x^{2}} \cdot d x$ for $\tan \theta^{\prime}-\tan \theta$, we get $T \frac{d^{2} y}{d x^{2}} \cdot d x$ as the value of the net upward force acting on the portion $a b$ of the string, and this net upward force is equal to the product of the mass, $m \cdot d x$, of the portion and the upward acceleration, $\frac{d^{2} y}{d t^{2}}$, of the portion. Therefore we have:

$$
m \frac{d^{2} y}{d t^{2}}=T \frac{d^{2} y}{d x^{2}}
$$

or

$$
\frac{d^{2} y}{d t^{2}}=\frac{T}{m} \frac{d^{2} y}{d x^{2}}
$$

This equation is identical with equation (II) of Art. II4 and its general solution is:

where

$$
y=F(x-v t)+f(x+v t)
$$

$$
v=\sqrt{\frac{T}{m}}
$$

The term $F(x-v t)$ represents a wave (a bend of any shape) traveling along the string from left to right, and the term $f(x+v t)$ represents a wave (a bend of any shape) traveling along the string from right to left; the velocity of travel being $\sqrt{\frac{T}{m}}$ cencimeters per second in each case.

Another point of view.-It is evident from Art. I I 4 that travel, pure and simple, is about the only thing that is established by the solution of equation (I) above. Therefore one might expect to obtain a perfectly clear and simple insight into the motion of a stretched string by introducing the idea of travel at the 
beginning. With this end in view let us consider a bend of any shape, and let us imagine that this bend is traveling along the string to the right (or left) at any velocity $v$. For example, one can make a bend of definite shape travel along a stretched string by threading the string through a bent tube and moving the bent tube along; but the state of affairs would be entirely unchanged if we imagine the bent tube to be stationary and the stretched string to be drawn through it, as indicated in Fig. I38. It is assumed

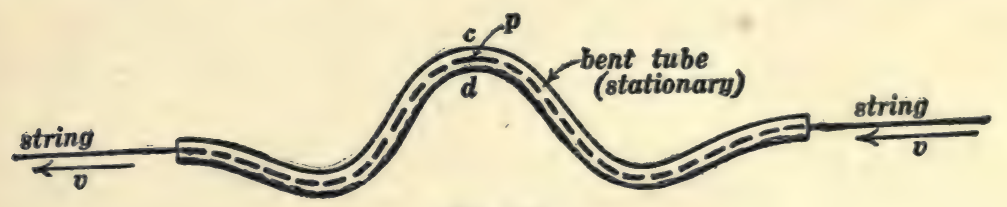

Fig. 138.

that the string slides through the tube without friction. The tension of the string alone would push the string against side $d$ of the tube at any point $p$, and the motion of the string (in the absence of tension) would throw the string against side $c$ of the tube because of centrifugal action. Let $r$ be the radius of curvature of the bent tube at $p$. Then the particles of the moving string may be thought of as traveling along a circular path of radius $r$ as they pass the point $p$. Therefore* the sidewise acceleration (in the direction of the radius $r$ ) of the string at $p$ is $\frac{v^{2}}{r}$. Consider a very short piece of the string of length $d s$ and whose mass is $m \cdot d s$. Then $m \cdot d s \times \frac{v^{2}}{r}$ is the side force (towards the center of curvature of the bent tube at $p$ ) which must act on the short piece of string to produce the specified acceleration $\frac{v^{2}}{r}$. If the string has no tension, then all of this side force is exerted by the side $c$ of the tube.

The stretched string near $p$ is like a barrel hoop under tension, and its tension $T$ produces on the element $d s$ a side force (draw-

* See Franklin, MacNutt and Charles's Calculus, Art. 50, page 70. 
ing it towards the center of curvature of the bent tube at $p$ ) equal to $\frac{T}{r} \cdot d s^{*}$; and if the string is not moving this force is exerted against the side $d$ of the tube.

If, however, the string is moving at a velocity $v$ which satisfies the equation:

or, if :

$$
m \frac{v^{2}}{r} \cdot d s=\frac{T}{r} \cdot d s
$$

$$
v=\sqrt{\frac{T}{m}}
$$

then the radial force (in the direction of $r$ ) due to the tension of the string is just sufficient to produce the necessary acceleration, and no force at all is exerted on the string by the guiding tube. Under these conditions the guiding tube might be removed and the bend would continue to stand in a fixed position on the traveling string, or if the string were standing still the bend would travel along the string at velocity $v$.

II6. Differential equations of electrical wave motion on a transmission line.-The theory of electrical wave motion is usually developed in terms of electric and magnetic field intensities in space, $\dagger$ and the equations are not easy to understand, especially by the electrical engineer who is accustomed to express everything in terms of voltage and current. Therefore the following discussion of electric wave motion on a transmission line is expressed in terms of voltage and current.

Throughout the following discussion the resistance of the line wires is assumed to be negligible and the line wires are assumed to be perfectly insulated.

* See Franklin, MacNutt and Charles's Calculus, Art. 51, page 72.

† A very simple development of this general theory is given on pages $186-195$ of Franklin's Electric Waves, The Macmillan Co., I909. The student must be familiar with the elements of vector analysis to be able to understand this electromagnetic theory. See Electric Waves, pages 158-185, or see Franklin, MacNutt and Charles's Calculus, pages 210-253. 
The horizontal lines in Fig. I39 represent the wires of a transmission line, and the heavy portions represent an element of the line. Let $e$ be the voltage across the transmission line at the

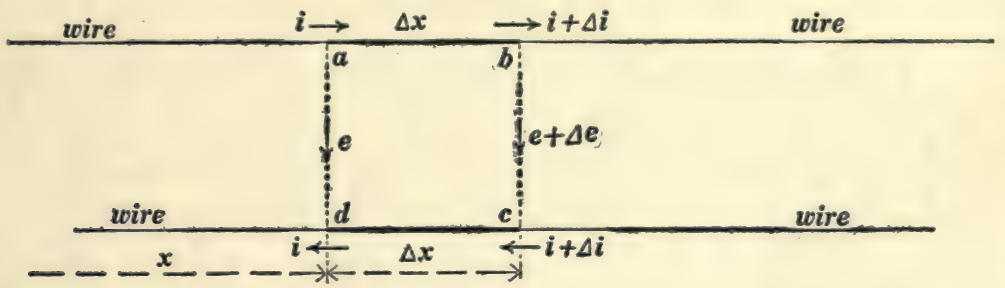

Fig. 139.

point $a d$ and let $i$ be "the current in the line at the same point" (meaning outflowing current in one wire and returning current in the other wire) as shown in Fig. 139. The voltage across the line at the point $b c$ is $e+\Delta e$, and the current in the line at the same point is $i+\Delta i$.

The capacity of the element $a b c d$ is $C \cdot \Delta x$, where $C$ is the capacity of unit length of the line. Therefore the charge $q$ "on the element" (positive charge on $a b$ and negative charge on $c d)$ is $q=C \cdot \Delta x \times e{ }^{*}$ and the rate of decrease of $q$, namely, $-\frac{d e}{d t} \cdot C \cdot \Delta x$, is equal to $\Delta i$. Therefore we have:

$$
C \frac{d e}{d t}=-\frac{d i}{d x}
$$

The net electromotive force around the elementary circuit $a b c d$ is $(e+\Delta e)$ minus $e$, and this electromotive force causes the current in the circuit to decrease ${ }^{2}$ at a definite rate such that $\Delta e=-L \cdot \Delta x \times \frac{d i}{d t}$ according to Art. 33 of Chapter III, where

* The charge is greater than $C . \Delta x \times e$ and less than $C . \Delta x \times(e+\Delta e)$ and when $\Delta x$ approaches zero, the expression for $q$ approaches $C . \Delta x \times e$ as a limit.

$\dagger$ The electromotive force $e+\Delta e$ is associated with an electric field from wire to wire and the arrow shows the direction of this field or the direction of $e+\Delta e$ as it would be indicated by a voltmeter. Evidently, however, an excessive charge on the wires at $b c$ and a large electromotive force from $b$ to $c$ would tend to create a current opposite to $i$. 
$L$ is the inductance of unit length of the line and $L \cdot \Delta x$ is the inductance of the elementary circuit $a b c d$. Therefore we have:

$$
L \frac{d i}{d t}=-\frac{d e}{d x}
$$

Equations (I) and (2) contain the two unknown dependent variables $e$ and $i$, and it is necessary to eliminate one to get an equation involving the other alone. By differentiating equation (I) with respect to $t$ and equation (2) with respect to $x$ we get:

$$
C \frac{d^{2} e}{d t^{2}}=-\frac{d^{2} i}{d x \cdot d t} \text { and } L \frac{d^{2} i}{d t \cdot d x}=-\frac{d^{2} e}{d x^{2}}
$$

But $\frac{d^{2} i}{d x \cdot d t}=\frac{d^{2} i}{d t \cdot d x}$; therefore we get:

$$
\frac{d^{2} e}{d t^{2}}=\frac{\mathrm{I}}{C L} \frac{d^{2} e}{d x^{2}}
$$

By differentiating equation (2) with respect to $t$ and equation (I) with respect to $x$ and eliminating as before, we get:

$$
\frac{d^{2} i}{d t^{2}}=\frac{\mathrm{I}}{C L} \frac{d^{2} i}{d x^{2}}
$$

The general solution of these equations establishes little besides the simple idea of travel, and if we introduce this idea at the start we can get a clear idea of the electric wave very much as we obtained a clear idea of a wave on a string by the discussion at the end of Art. II5.

Another point of view.-Imagine current to be distributed over a transmission line in any arbitrary manner, the current $i$ at any given point ad of the line (outflowing current in one wire, returning current in the other wire) being represented by the ordinate $i$ of any given curve $C C$, Fig. I40, and suppose this current distribution as a whole to travel to the right in Fig. I40 at velocity $V$. Such a traveling current distribution would produce a voltage distribution over the line such that:

$$
e=\operatorname{LiV}
$$


where $e$ is the voltage across the line at the place where the current in the line is $i$. This equation may be established as follows: The inductance of the element $a b c d$ is $L \cdot \Delta x$, and the magnetic flux between the wires $a b$ and $c d$ is equal to the inductance of the element multiplied by the current, everything being expressed in c.g.s. units. Now, the current distribution and the associated flux are assumed to travel to the right at velocity $V$ so that all of the flux between $a b$ and $c d$ will sweep across the line $b c$ in $\Delta x / V$ of a second. Therefore the voltage $e$ induced along $b c$ by the traveling flux is $L i \cdot \Delta x$ divided by $\Delta x / V$ which gives $L i V$ abvolts.

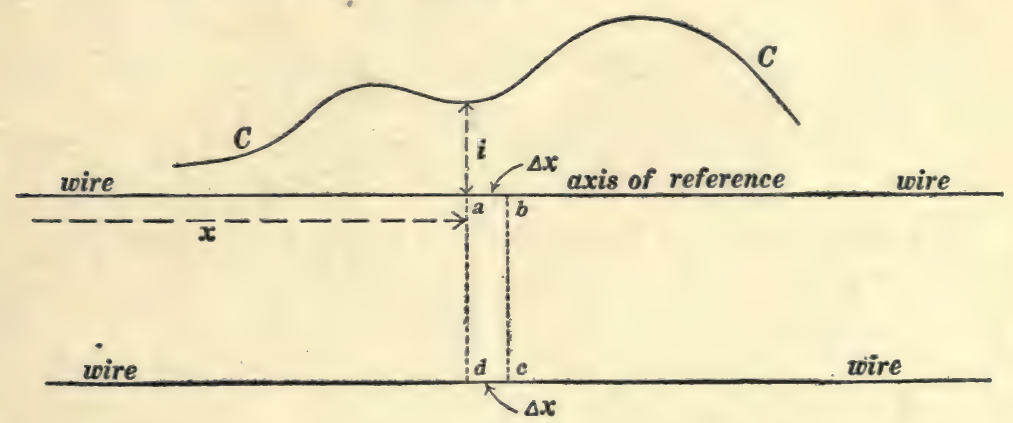

Fig. 140.

Imagine electric charge to be distributed over the transmission line in Fig. I40 (positive charge on one wire and an equal negative charge on the other). This charge means a definite voltage between wires at each point along the line, and we may imagine the voltage $e^{\prime}$ at each point to be represented by the ordinate of a curve $C C$. Imagine the electric charge and the associated voltage distribution to travel along the line at velocity $V^{\prime}$. Such a traveling voltage distribution would produce a definite current distribution over the line such that

$$
i^{\prime}=C e^{\prime} V^{\prime}
$$

where $i^{\prime}$ is the current in the line at the place where the voltage across the line is $e^{\prime}$, and $C$ is the capacity of the line per unit 
of length. This equation may be established as follows: The capacity of the element abcd is $C \cdot \Delta x$ so that $C \cdot \Delta x \times e^{\prime}$ is the charge on $a b$ (or negative charge on $c d$ ). But all of this charge must flow past the point $b$ during $\Delta x / V^{\prime}$ of a second, so that the current in the line is found by dividing $C \cdot \Delta x \times e^{\prime}$ by $\Delta x / V^{\prime}$ which gives equation (6).

Imagine a current distribution and a voltage distribution traveling along together and each sustaining the other according to equations (5) and (6), then $i=i^{\prime}, e=e^{\prime}$, and $V=V^{\prime}$ so that equation (6) may be written:

$$
i=\mathrm{CeV}
$$

Solving equations (5) and ( 7 ) for $V$, we get

$$
V=\sqrt{\frac{\mathrm{I}}{L C}}
$$

And eliminating $V$ from equations (5) and (7), we get

$$
\frac{1}{2} L i^{2}=\frac{1}{2} C e^{2}
$$

This equation means that the magnetic energy per unit length of line $\left(\frac{1}{2} L i^{2}\right)$ is equal to the electric energy per unit length of line $\left(\frac{1}{2} C e^{2}\right)$ in a wave of the kind which is represented by equations (5) and (7).

Equation (8) gives the velocity at which two mutually sustaining current and voltage distributions must travel, and two such mutually sustaining distributions of $e$ and $i$ constitute an electromagnetic wave. If $L$ is expressed in henrys per mile (see Art.39), and if $C$ is expressed in farads per mile (see Art. 104), then equation (8) gives $V$ as 186,000 miles per second.

The voltage and current distributions in a traveling wave must be alike according to equations (5) and ( 7$)$; that is, where $i$ is large, $e$ is correspondingly large. The relation between $e$ and $i$ is shown best by equation (9) from which we get

$$
\frac{e}{i}= \pm \sqrt{\frac{L}{C}}
$$


The plus sign relates to a wave moving to the right in Fig. I40, and the negative sign relates to a wave moving to the left in Fig. I40.

A clear understanding of an electric wave traveling along a transmission line may be obtained from Fig. I4I, resistance of

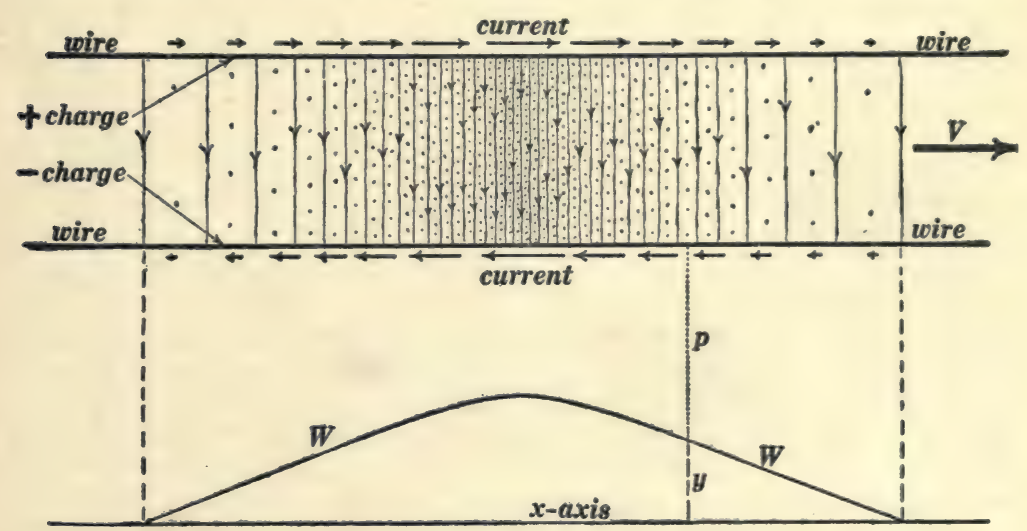

Fig. 141.

Showing an electromagnetic wave on a transmission line.

line wires being negligible and wires being perfectly insulated. The curve $W W$ corresponds to $C C$ in Fig. I40, and the ordinate $y$ represents the voltage $e$ across the line or the current $i$ in the line at the point $f$. The upper wire is positively charged and the fine vertical lines represent the lines of force of the electric field which emanates from the positively charged wire and converges upon the lower wire in the figure which is negatively charged. Current flows to the right in the upper wire and to the left in the lower wire as represented by the short horizontal arrows, and the fine dots represent the lines of force of the magnetic field between the wires, this magnetic field being perpendicular to the plane of the paper in Fig. I4I. The heavy arrow $V$ shows the direction of travel of the wave.

The direction of travel and the directions of $e$ and $i$ may be correlated as follows: That particular wire is positively charged out of which the electric lines of force emanate; the voltage $e$ is 
from positively charged wire to negatively charged wire; and the current in the positively charged wire may be thought of as carrying the positive charge forwards from the back of the wave and laying it down on the wire in front of the wave.

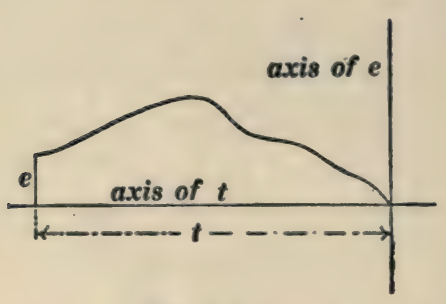

Fig. 142.

Showing voltage $e$ as a function of the time.

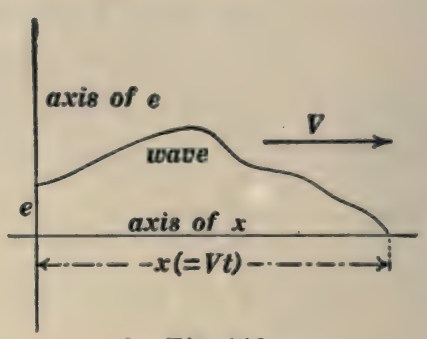

Fig. 143.

Showing shape of wave which shoots out from the end of a transmission line when the voltage of Fig. $\mathbf{I}_{42}$ is connected across the end of the line.

The wave which is represented in Fig. I4I is called an isolated wave or a wave pulse. . Such a wave is produced when an electromotive force is connected across the end of the transmission line for a moment and then disconnected. The relation between the voltage across the end of the line and the shape of the wave produced thereby is shown in Figs. I42 and I43. The ordinates of the curve in Fig. 142 represent the varying value of the voltage $e$ across the end of the line as a function of elapsed time $t$, time being measured to the left along the axis of abscissas. The ordinates of the exactly similar curve in Fig. I43 represent the values of $e$ and $i$ in the wave which shoots out on the line. The line is assumed to be very long so that no complications arise from the reflection of the wave from the distant end of the line. That Fig. 143 shows the actual wave produced when the varying voltage of Fig. $\mathrm{I}_{42}$ is connected across the end of the line is evident from the following considerations: A particular solution of any differential equation must satisfy the equation itself throughout the region to which the solution applies, and it must satisfy the conditions which are impressed at the bound- 
aries to the region. Thus the wave, Fig. I43, which shoots out on the line when the varying voltage of Fig. 142 is connected across the end of the line satisfies the differential equations (3) and (4) because travel, pure and simple, is all that these equations demand; and it satisfies the boundary conditions at the end of the line as is evident from the identity of the curves in Figs. 142 and 143 . It must be remembered that the effects of wire resistance and of imperfect insulation between line wires are ignored in this discussion.

When a periodic electromotive force acts across the end of the line, for example when an alternator is connected to the line, then what is called a wave-train passes out along the line, and the state of affairs (before matters are complicated by the reflection of the waves from the distant end of the line) is shown in Fig. 144. This figure shows a wave-train which is produced by a

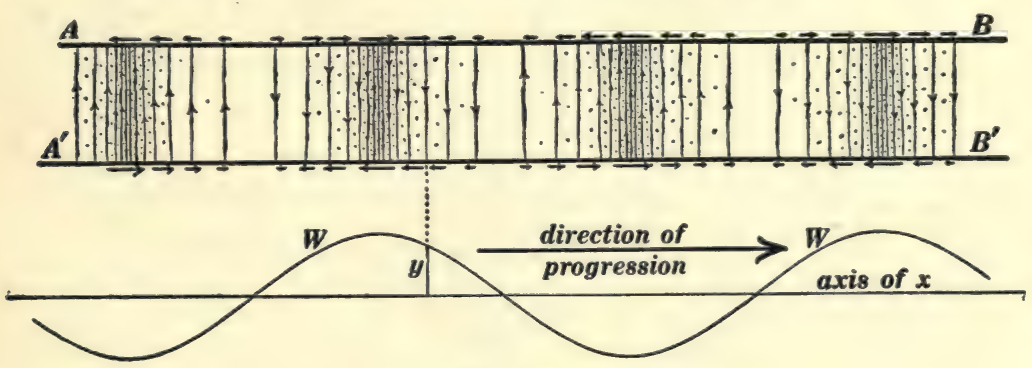

Fig. 144.

harmonic alternating voltage, and the curve $W W$ is a curve of sines. The short horizontal arrows represent the current at various places in the wires, the fine vertical lines represent the lines of force of the electric field, and the dots represent the lines of force of the magnetic field as in Fig. I4I.

The ribbon wave.-When a battery of constant voltage and negligible resistance is connected across the end of a transmission line, a wave shoots out on the line, the voltage $e$ in the wave is everywhere of the same value and equal to battery voltage, and the current is everywhere the same in value and equal to 
$\sqrt{\frac{L}{C}} \times e$. Such a wave we will call a ribbon wave. Thus Fig. I45 represents a ribbon wave $d / V$ seconds after the battery is

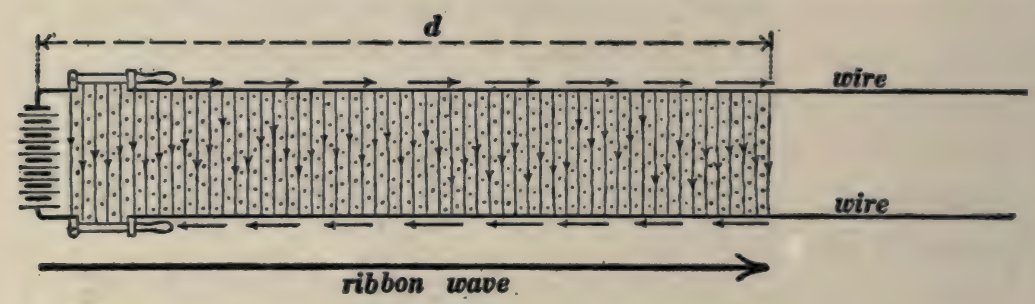

Fig. 145.

Showing the ribbon wave which shoots out from a battery which is suddenly connected to the end of a line.

connected. The short horizontal arrows represent current, the fine vertical lines represent the lines of force of the electric field, and dots represent magnetic lines of force as in Figs. I4I and I44. The rectangular wave pulse.-Figure 146 represents the iso-

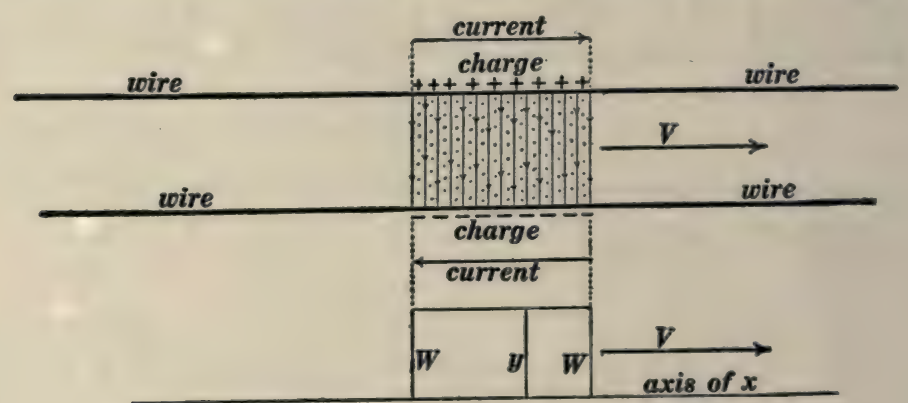

Fig. 146.

A rectangular electromagnetic wave pulse.

lated wave pulse which is produced when a battery of negligible resistance is connected across the end of the line for a moment and then disconnected. The ordinate $y$ of the curve $W W$ represents the voltage $e$ or the current $i$ at each point on the line, and the wave pulse is called a rectangular wave pulse because this curve $W W$ is a rectangle. 
II7. Superposition of waves--A principle of extremely wide application in physics is the so-called principle of superposition. From the physical point of view a general statement of the principle of superposition is scarcely possible and therefore the following examples must suffice:

(a) A person at $A$, Fig. 147 , can see window No. I and another person at $B$ can see window No. 2 at the same time. This means that two beams of light $a$ and $b$ can travel through the same region at the same time without getting tangled up together, as it were, each beam behaving as if it were traveling through the region alone. (b) Two systems of water waves can travel over the same part of a pond simultaneously, each system behav-

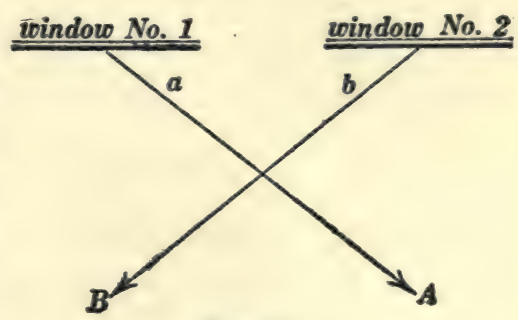

Fig. 147. ing as if the other system were non-existent. (c) Two forces $F$ and $G$ exerted together on an elastic structure produce a deformation which is the sum of the deformations which would be produced by the forces separately, provided the two forces together do not exceed the elastic limit of the structure. Therefore each force may be thought of as producing the same effect that it would produce if it were acting alone.

All of the effects in physics which are thus superposableand this includes the majority of effects in mechanics, heat, electricity and magnetism, light and sound-are expressible in terms of linear differential equations. Thus equations (I), (2), (3) and (4) of Art. I I6 are linear differential equations, and any number of electric waves can travel over a transmission line simultaneously, each behaving as if it were alone. The voltage across the line at any point is the sum of the voltages at that point due to the separate waves, and the current in the line at a point* is the sum of the currents at that point due to the separate waves.

* Outflowing current in one wire and returning current in the other wire. 
II8. Reflection.-For the sake of brevity and clearness we will discuss only the reflection of rectangular wave pulses, and such a wave pulse will be represented by a single heavy horizontal arrow. When such a wave is turned back or reflected at the end of a line, the heavy arrow will be shown as turned back as in Figs. 148, I49 and 150. The voltage and current in the original wave are represented by $E$ and $I$ and the voltage and current in the reflected wave are represented by $E_{r}$ and $I_{r}$ as shown.

Reflection from open end of line.-The doubled arrow in Fig. I48 represents a wave which has been turned back or reflected
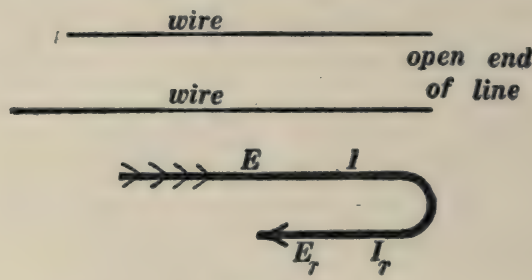

Fig. 148.

Showing a rectangular wave pulse partly reflected from the open end of a line.

at the open end of a transmission line. The necessary condition which must be satisfied at the open end of a line is that the actual current there be zero. Therefore we have:

$$
I+I_{r}=0
$$

In addition to this we must have:

$$
\frac{E}{I}=+\sqrt{\frac{L}{C}}
$$

and

$$
\frac{E_{r}}{I_{r}}=-\sqrt{\frac{L}{C}}
$$

Now the voltage and current in the original wave are supposed to be given and the voltage and current in the original wave do, as a matter of course, satisfy equation (2). From equation (I) we have: 
ELECTRIC OSCILLATIONS AND ELECTRIC WAVES. 213

$$
I_{r}=-I
$$

and from equations (2) and (3) we have:

$$
E_{r}=+E
$$

Reflection at the open end of a line is complete and it takes place with reversal of current.

Reflection from short-circuited end of line.-The doubled arrow in Fig. I49 represents a wave which has been turned back
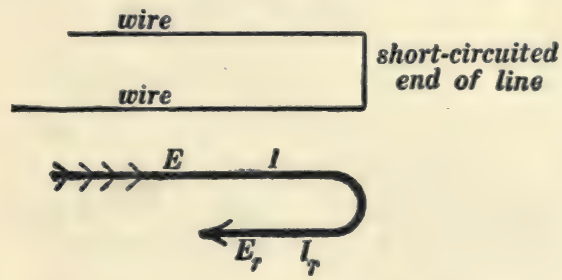

Fig. 149.

Showing a rectangular wave pulse partly reflected from the short circuited end of a line.

or reflected from the short-circuited end of a line. The necessary condition which must be satisfied at the short-circuited end of a line is that the actual voltage across the end be zero. Therefore we have:

whence

$$
E+E_{r}=0
$$

$$
E_{r}=-E
$$

and, since equations (2) and (3) always apply, we get:

$$
I_{r}=+I
$$

Reflection at a short-circuited end of a transmission line is complete and it takes place with reversal of voltage.

Reflection from a non-inductive circuit connected across the end of a transmission line.*-The doubled arrow in Fig. I50 represents a wave which has been turned back or reflected from

* Reflection from inductive circuits is discussed in a paper by W. S. Franklin, Proceedings American Institute of Electrical Engineers, April, I9I4. 
the end of a line which is connected to a non-inductive circuit of resistance $R$. The actual current at the end of the line is $I+I_{r}$ and this current flows through $R$ so that the voltage across the
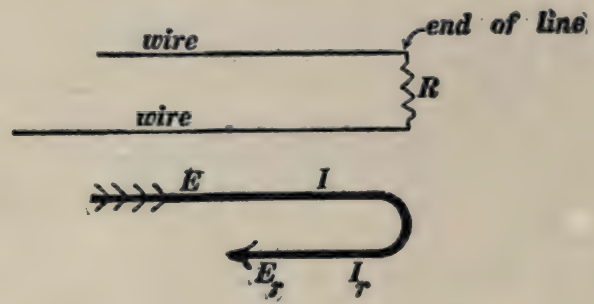

Fig. 150.

Showing a rectangular wave pulse partly reflected from the non-inductive receiving circuit of resistance $R$.

end of the line $\left(E+E_{r}\right)$ must be equal to $R\left(I+I_{r}\right)$. Therefore we have:

Also we have:

$$
E+E_{r}=R\left(I+I_{r}\right)
$$

$$
\frac{E}{I}=+a
$$

and

$$
\frac{E_{r}}{I_{r}}=-a
$$

where $a$ is written for $\sqrt{\frac{L}{C}}$. Solving equations (9), (10) and (I I) we get:

and

$$
E_{r}=E\left(\frac{R-a}{R+a}\right)
$$

$$
I_{r}=I\left(\frac{a-R}{a+R}\right)
$$

(a) When $R=a$ there is no reflection at all; the entire wave is swallowed up, as it were, by the circuit $R$. (b) When $R$ is greater than $a$ there is partial reflection with reversal of current. (c) When $R$ is less than a there is partial reflection with reversal of voltage. 
For example, let $R=3 a$, then $E_{r}=+E / 2$, and $I_{r}=-I / 2$. When $R=a / 3$, then $E_{r}=-E / 2$ and $I_{r}=+I / 2$.

Reflection at a place where the line constants $L$ and $C$ change. - The doubled arrow in Fig. 15I represents a wave which

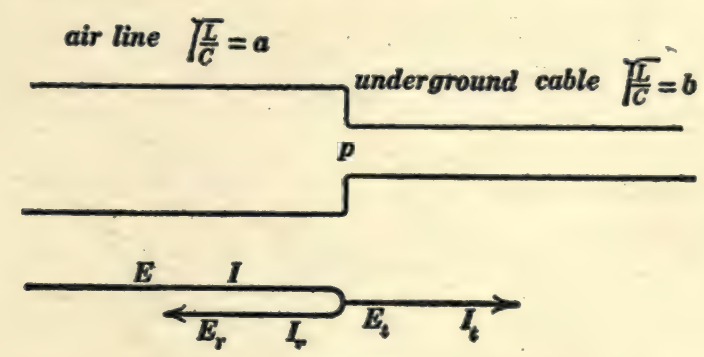

Fig. 151.

Showing a rectangular wave pulse partly reflected and partly transmitted at a point where an air line connects with an underground cable.

has been partly turned back or reflected at a place where an air line connects to an underground cable, and the arrow $E_{t} I_{t}$ represents the wave which enters the cable. In this case we have:

$$
\begin{gathered}
E+E_{r}=E_{t} \\
I+I_{r}=I_{t} \\
\frac{E}{I}=+a \\
\frac{E_{r}}{I_{r}}=-a \\
\frac{E_{t}}{I_{t}}=+b
\end{gathered}
$$

and from these equations we find:

$$
\begin{aligned}
& E_{r}=\frac{b-a}{b+a} \cdot E \\
& I_{r}=\frac{a-b}{a+b} \cdot I
\end{aligned}
$$




$$
\begin{aligned}
& E_{t}=\frac{2 b}{a+b} \cdot E \\
& I_{t}=\frac{2 a}{a+b} \cdot I
\end{aligned}
$$

Reflection at a place where a line branches.-The doubled arrow in Fig. 152 represents a wave which has been partly

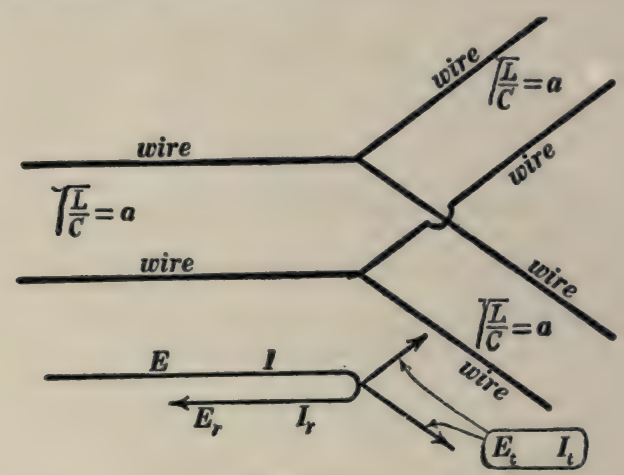

Fig. 152.

Showing a rectangular wave pulse partly reflected and partly transmitted at a point where an air line branches.

reflected at a branch point in a line, and the two short arrows $E_{t} I_{t}$ represent the waves which have entered the branches. The two branches are both assumed to be like the original line for the sake of simplicity. In this case we have:

$$
\begin{gathered}
E+E_{r}=E_{t} \\
I+I_{r}=2 I_{t} \\
\frac{E}{I}=+a \\
\frac{E_{r}}{I_{r}}=-a \\
\frac{E_{t}}{I_{t}}=+a
\end{gathered}
$$

from which we get $E_{r}=-E / 3$ and $I_{r}=+I / 3 ;$ and $E_{t}=+\frac{2}{3} E$ 
and $I_{t}=+\frac{2}{3} I$. This is, of course, a very special problem because both branches are exactly like the main line, and in this case one ninth of the energy of the original wave is reflected back on the main line and four ninths of the energy of the original wave enters each branch.

IIg. Transmission line surges which follow the switching on of a generator.-When a generator of negligible resistance and

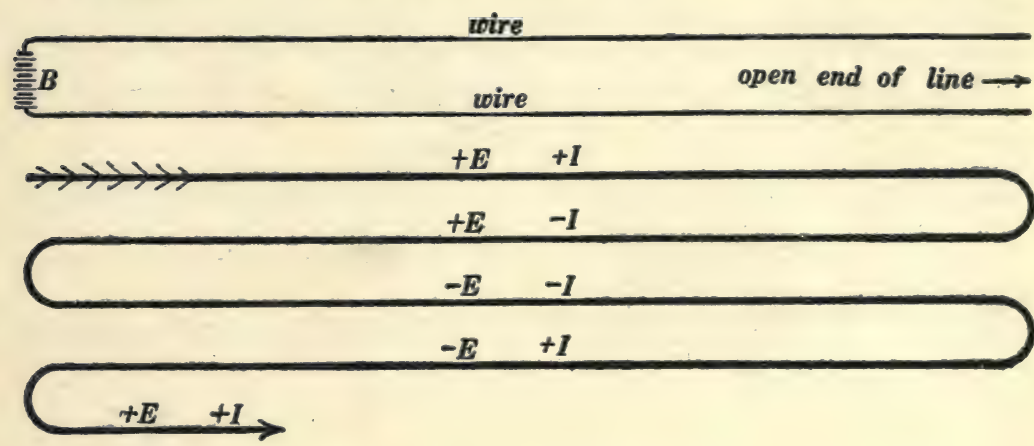

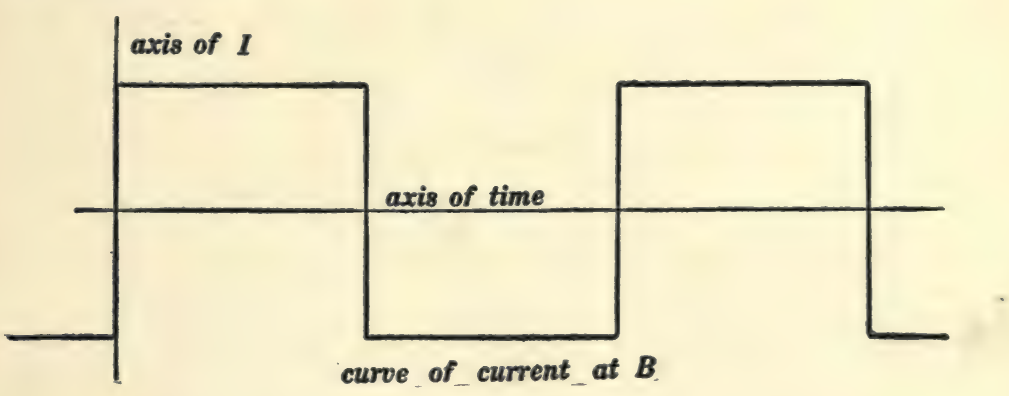

Fig. 153.

The upper part of the figure shows a ribbon wave which shoots out from a suddenly connected generator and is repeatedly reflected at both ends of the line. The lower part of the figure shows the value of the current at $B$ as a function of elapsed time.

inductance is suddenly switched on to a line, a ribbon wave of generator voltage and corresponding current shoots out over the line. Assuming line resistance to be negligible and line insulation to be perfect, this ribbon wave is reflected back and forth as represented in Figs. I53, I54 and I55, and by adding voltages 
and currents in the successive laps of the ribbon wave a precise knowledge of the distribution of voltage and current over the line at any instant may be obtained. Thus, after a thousandth of a second, the total length of the ribbon wave would be 186

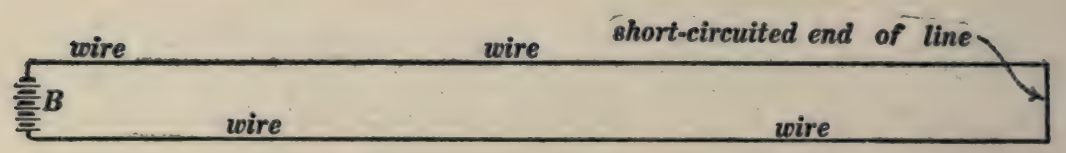

\begin{tabular}{rrr}
\hline$H$ ribbon wave & $+E$ & $+I$ \\
\hline$-E$ & $+I$ \\
\hline$+E$ & $+I$ \\
\hline$-E$ & $+I$
\end{tabular}

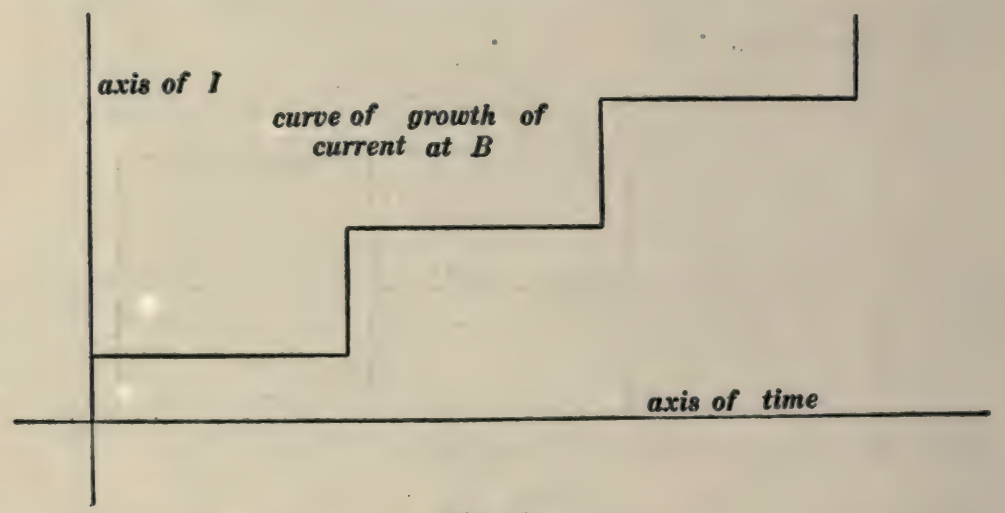

Fig. 154.

Same as Fig. I53 except that distant end of line is short-circuited.

miles which would give a definite number of complete laps and a fraction of the next lap. By adding a number of laps in this way, curves can be plotted showing the actual distribution of voltage and current over the line at any given instant; or values of current and voltage at a given point of the line can be found for successive instants and from this data curves can be plotted 
showing voltage or current at any point as functions of elapsed times. The ampere-time curves in the lower parts of Figs. I53, I54 and I55, and in Figs. I56 and I57 were obtained in this way.

\begin{tabular}{ccc}
\multicolumn{2}{c}{ wire } & wire \\
奒 $B \quad$ wire & wire
\end{tabular}

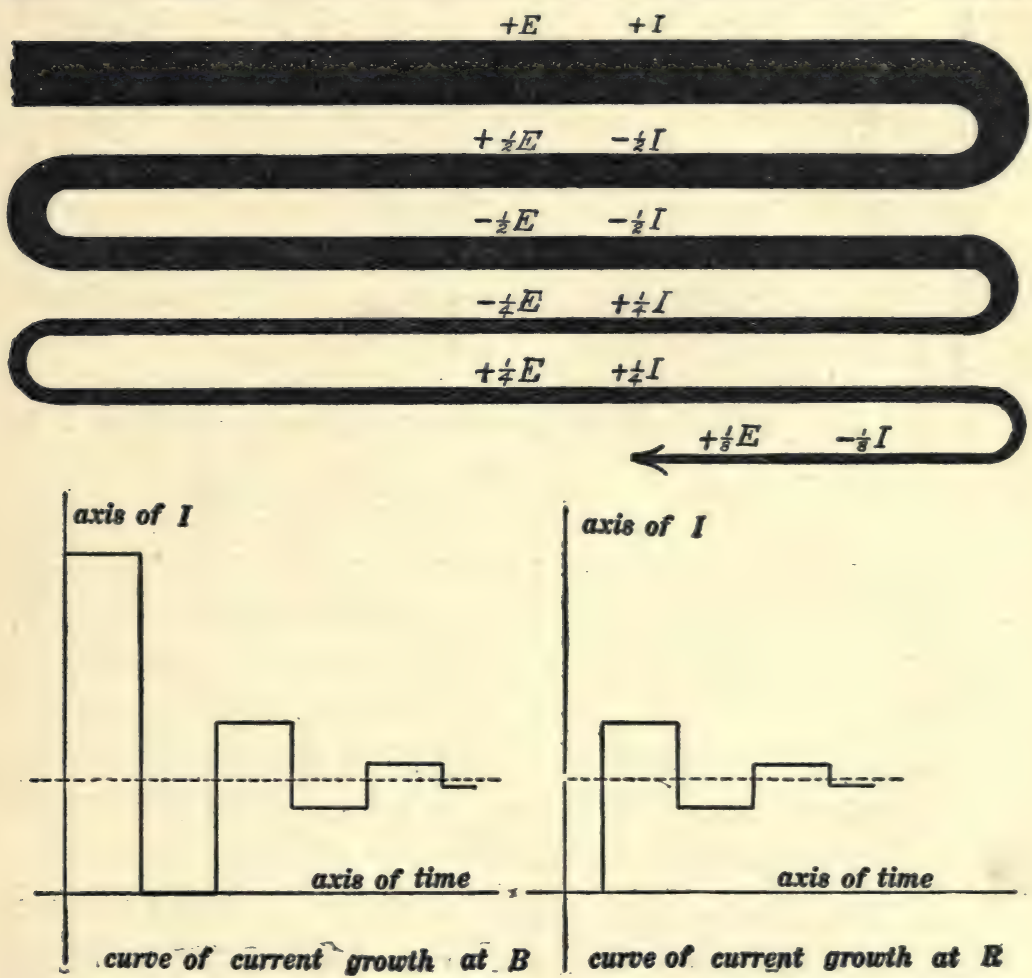

Fig. 155.

Same as Fig. I53 except that distant end of line is connected to a non-inductive circuit of which the resistance is $R=3 \sqrt{\frac{L}{C}}$. In this case the ribbon wave is only partially reflected from the end $R$ and the successive laps of the ribbon wave are thereby greatly reduced in intensity as represented by the shading.

Note.-The character of the reflection at $B$ in Figs. I53, I54 and $\mathbf{I} 55$ is determined by the condition that the sum of the vol- 
tages in the successive laps must always be equal to battery voltage $E$, and of course there is always an odd number of laps at $B$, one, or three, or five, etc.

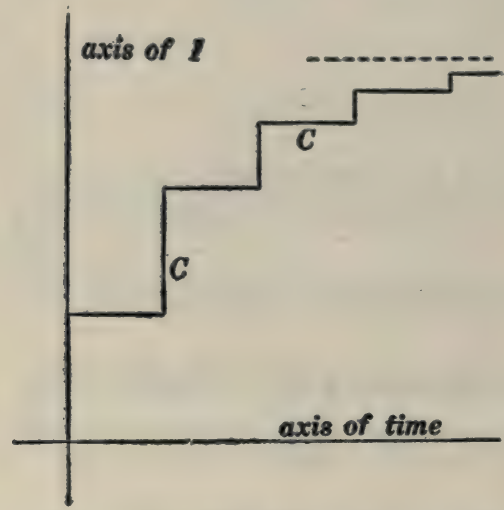

Fig. 156.

Showing growth of current at $B$ in Fig. 155 when $R=\frac{\mathrm{I}}{3} \sqrt{\frac{\bar{L}}{C}}$.

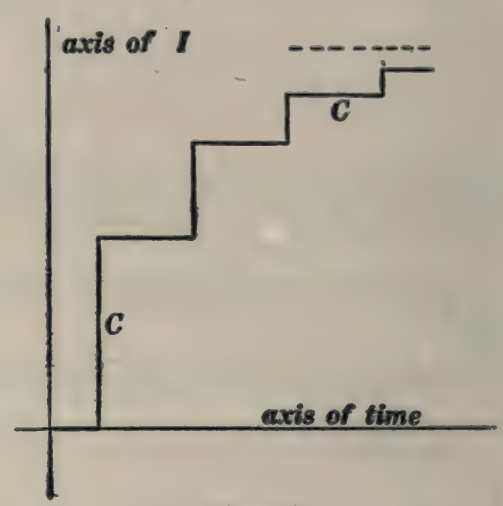

Fig. 157.

Showing growth of current at $R$ in Fig. I55 when $R=\frac{I}{3} \sqrt{\frac{L}{C}}$.

Note.-Figures 153,154 and 155 show what takes place when a direct-current generator or battery is switched on to the line, but on a line of moderate length a number of laps are formed in an excessively short interval of time, and therefore Figs. 153, 154 and I55 show quite accurately what takes place immediately after an alternator is switched on to the line, $E$ being the value of the voltage of the alternator at the instant of closing the switch.

I20. Transmission line surges which are produced when a circuit breaker opens. - When a circuit breaker opens, the arc which is formed persists for a very long time, relatively speaking, and the open gap in the circuit is filled with a fairly good conducting material which slowly loses its conductivity. It is about as nearly impossible to produce characteristic line surges by opening a circuit breaker as it would be to set up an abrupt water wave in a canal by allowing a cubic mile of soft mud to flow into the canal prism to stop a troublesome flow of water in the canal; and yet the moon, as a 60 cycle generator ( 60 cycles per month!), 
might produce a troublesome tidal wash in a large estuary while the attempt was being made to "open-circuit" the estuary in this Brobdignagian fashion! Let the reader consider this hydraulic analog carefully. It reproduces nearly all of the essentials of the electrical case. The conducting vapor in the arc of a circuit breaker is somewhat analogous to mud as a dam building material.

Very little need be said of characteristic line surges in connection with opening of switches, except in the case of very long lines. When a line is short, or only moderately long, what takes place may be described quite accurately in terms of the simple ideas of the elementary alternating-current theory, where current values are supposed to rise and fall simultaneously throughout an entire
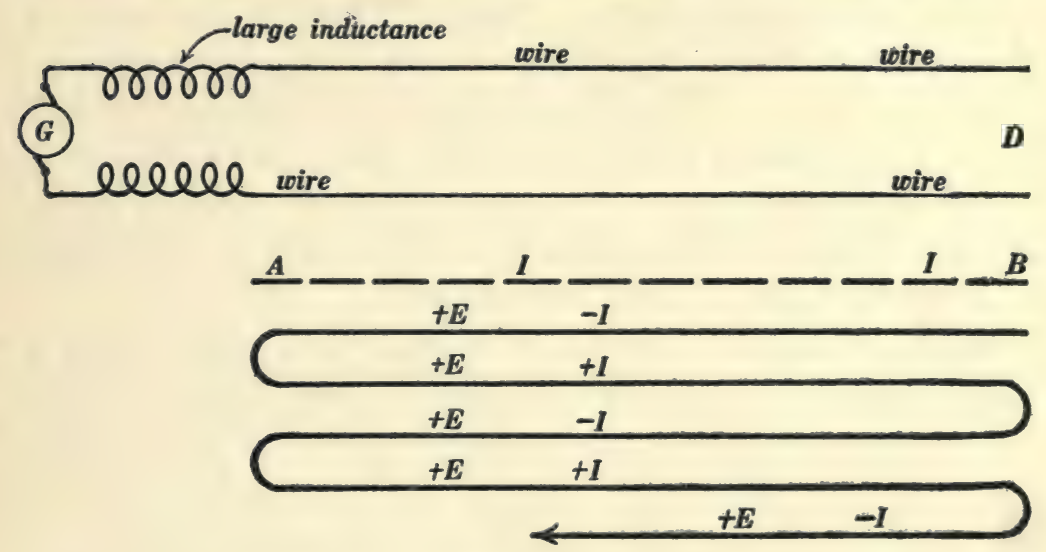

Fig. 158.

The dotted line $A B$ represents the initial current in the transmission line. The long heavy arrow with laps represents the ribbon wave which comes from $D$ after the line at $D$ is opened.

circuit. The formation of a long arc between line wires in air and the quick snapping out of such an arc does, however, produce an electric wave disturbance on a transmission line of moderate length, and the essential features of this case are shown in Fig. 158. Imagine the system to be short-circuited by an arc at the distant end $D$ of the transmission line, voltage being reduced to a 
negligibly small value over the whole line, and a large current $I$ being established in the line. The generator is to be thought of as having a large inductance so that the generator current cannot change perceptibly during the very short time required for the characteristic line surges. When the distant end of the line is opened, a ribbon wave shoots towards the generator, is completely reflected at the generator with reversal of current, again completely reflected at the opened end $D$ of the line with reversal of current, and so on. The first lap of this ribbon wave wipes out the current in the line and lays down a certain voltage $E$ $\left(=\sqrt{\frac{L}{C}}\right.$ times the initial value of the current). The second lap of the ribbon wave lays down a double voltage, and the original current. The third lap wipes out the current again, and lays down a voltage equal to $3 E$, and so on.

Note.-The character of the reflection at the generator in Fig. I $5^{8}$ is determined by the condition that the sum of the currents in the successive laps at $A$ must always be equal to the initial current $I$ because the current in the highly inductive generator does not have time to change perceptibly.

Note.-It must not be imagined that the sending out of a ribbon wave depends upon a continued supply of energy at the point where the ribbon wave originates. Thus in Fig. 158 the ribbon wave is superposed upon the initial current $I$, and the first lap of the ribbon wave wipes out this current. Therefore since the current is zero, there is no energy flow at all from the opened end. The second lap of the ribbon wave lays down the original current $I$ and a doubled voltage $2 E$, and this combination of voltage and current represents a flow of energy from the generator into the line.

I2I. Impure wave and pure wave.-A wave of the type which is represented by equations (5) and (7) of Art. I I6 travels along a transmission line without any change of shape, and in such a wave the magnetic energy per unit length of line $\left(\frac{1}{2} L i^{2}\right)$ is 
equal to the electric energy per unit length of line $\left(\frac{1}{2} C e^{2}\right)$, according to equation (9) or equation (10) of Art. 116. Such $a$ wave is called a pure wave.

When $\frac{1}{2} L i^{2}$ is not equal to $\frac{1}{2} C e^{2}$ we have what is called an impure wave, and an impure wave is always resolvable into two pure waves traveling in opposite directions. For example, the first two laps of the ribbon wave in Fig. I53 give voltage $=2 E$ and current equal to zero over the whole line, and this condition is resolvable into the two oppositely moving waves, namely, the first lap $(+E,+I)$ and the second lap $(+E,-I)$. This resolution may be made in any case as follows: Let $A$ be the voltage across the line and $B$ the current in the line at any point, and let it be required to resolve this state of affairs into a pure wave $E^{\prime} I^{\prime}$ traveling to the right and a pure wave $E^{\prime \prime} I^{\prime \prime}$ traveling to the left. Then we have

$$
\begin{gathered}
\frac{E^{\prime}}{I^{\prime}}=+a \\
\frac{E^{\prime \prime}}{I^{\prime \prime}}=-a \\
E^{\prime}+E^{\prime \prime}=A \\
I^{\prime}+I^{\prime \prime}=B
\end{gathered}
$$

where $a$ is written for $\sqrt{\frac{L}{C}}$. These four equations determine $E^{\prime}, E^{\prime \prime}, I^{\prime}$ and $I^{\prime \prime}$. Of course either $E^{\prime \prime}$ or $I^{\prime \prime}$ must be negative if both $E^{\prime}$ and $I^{\prime}$ are positive.

122. Immediate effects of wire resistance and poor insulation on a wave on a transmission line.-When an electric current dies away in an ordinary circuit of wire (after the electromotive force of a battery, for example, has ceased to act) the magnetic energy of the circuit (see Art. 35) is slowly converted into heat in the wire in accordance with Joule's law, and if we imagine that a pure wave has suddenly entered upon a transmission line of which the wire resistance is not negligible, then the immediate 
effect of the wire resistance is to cause the magnetic energy in the wave to decrease thus causing the wave to become impure.

When a charged condenser is left standing a very small current flows through the imperfectly insulating dielectric of the condenser and the electric energy of the condenser is slowly converted into heat in the insulating material, and if we imagine that a pure wave has suddenly entered upon a transmission line which is not perfectly insulated, then the immediate effect of the imperfect insulation is to cause the electrical energy in the wave to decrease, thus causing the wave to become impure.

Wave distortion.-Imagine a pure rectangular wave pulse to have entered a transmission line which has wire resistance. This wave is represented in Fig. I59. After this wave has traveled a

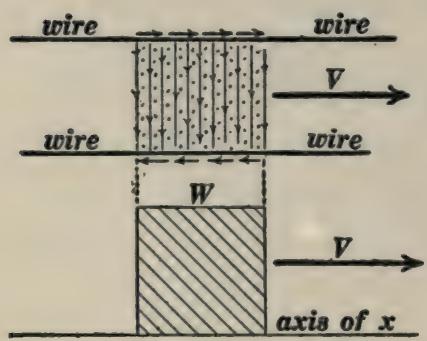

Fig. 159.

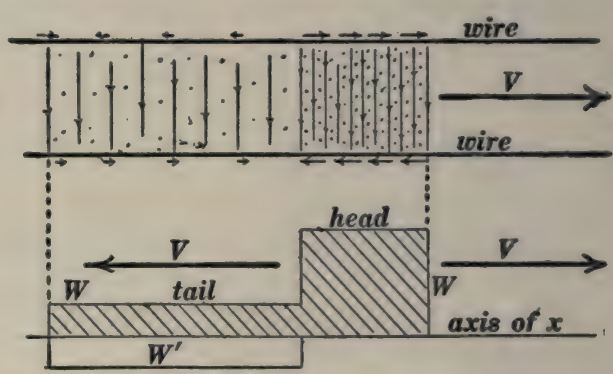

Fig. 160.

Fig. I59 represents a pure rectangular wave traveling along a transmission line, and Fig. 160 represents the state of affairs after an interval of time $\Delta t$ has elapsed. The development of a tail in Fig. I60 is due to wire resistance.

very short distance along the line, the magnetic energy in the wave will have been reduced by the effect of wire resistance and the wave will have become impure. This impure wave then breaks up into two pure waves, one of which, containing nearly all of the energy of the original wave, travels forwards, and the other travels backwards. This action is here described as taking place after the original wave has traveled a very short distance along the line, but in fact the action is continuous; the rectangular wave sends out backwards a long drawn out ribbon wave thus 


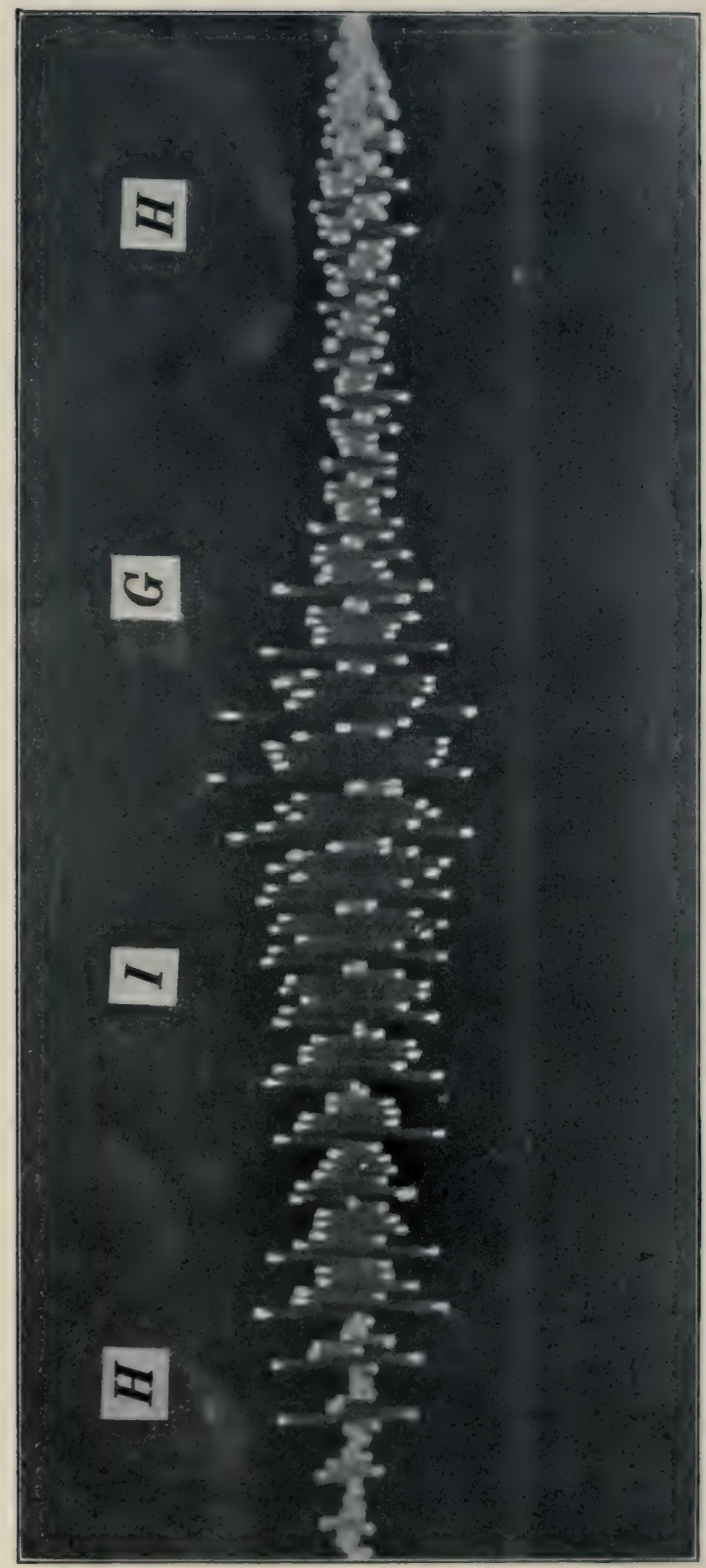

ฮั

"艹

㟧

क् है ह

气

is

วิ

क. 요

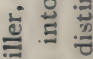

远

ن क्ष

A

. 해

๘ั

은 능

촌

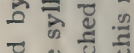

उ

ฮี

它合

म.

运

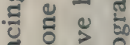

뜬 ปั

음

है 즐

땡요

ค กิ

혐

ก

ํㅐ용

웧 के

एँ क

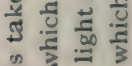

들 छ क

政

จ

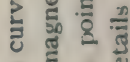

है है

ह 
devcloping a long tail, and the energy of the head of the wave decreases, because of loss of energy in heating the wires, and also because of the carrying away of energy by the long drawn out ribbon wave which constitutes the tail. The state of affairs after the original wave has traveled a short distance is shown approximately in Fig. I60, the initial state of affairs being shown in Fig. 159. The tail of the wave in Fig. I60 is traveling backwards as indicated, and the voltage in the tail is negative* as represented by the curve $W^{\prime}$.

When a rectangular wave pulse enters a line of which the wire resistance is negligible but which is poorly insulated, then the effect is very similar to what is shown in Fig. I60. In this case the electrical energy of the wave is reduced by line leakage and the wave develops a tail. Wire resistance causes a rectangular wave pulse to develop a tail in which voltage is reversed, and line leakage causes a rectangular wave pulse to develop a tail in which current is reversed.

The effect of wave distortion is very serious in long distance telephony. Thus the curve in Fig. I6I represents the complicated wave which enters a telephone line from the transmitter when the syllable high is spoken into the transmitter, and the reproduction of the original sound by the distant telephone receiver depends upon the delivery of this complicated wave with all of its fine detail at the distant end of the line. The effect of wire resistance (or the effect of poor insulation), however, is to cause each shortest portion of this complicated wave to spread over the line by the development of a tail as explained in connection with Fig. I6o. That is, a tail shoots out backwards from each shortest portion of the wave in Fig. 16I, and the result is the smoothing down of the sharp points and the filling in of the deep valleys of the curve so that all fineness of detail of the original wave shape is obliterated and nothing but a dull droning sound is produced in the distant telephone receiver.

* The tail of the wave in Fig. I60 may be thought of as a pure wave at the very beginning, but it soon becomes impure just as the head of the wave itself becomes impure. 
The distortionless line.-Let $I$ be the current and $E$ the voltage in a pure wave which enters a transmission line. Then the rate at which energy is lost in heating the wires of unit length of the line is $R_{w} I^{2}$, where $R_{w}$ is the resistance of the two wires in unit length of the line. Let $R_{l}$ be the leakage resistance between wires of unit length of line, then $E / R_{l}$ is the leakage current from wire to wire because of imperfect insulation, and $E^{2} / R_{l}$ is the rate at which energy is lost in unit length of the line because of imperfect insulation. Now $R_{w} I^{2}$ is the rate of loss of magnetic energy and $E^{2} / R_{l}$ is the rate of loss of electric energy in unit length of line, and if $R_{w} I^{2}=E^{2} / R_{l}$, then magnetic energy and electric energy are lost at the same rate; but electric and magnetic energies are equal at the start, therefore they remain equal and the wave remains pure if $R_{w} I^{2}=E^{2} / R_{l}$. On a line where this condition is satisfied there is no wave distortion, and such a line is therefore called a distortionless line. The condition is best expressed by substituting the value $\frac{L}{C}$ for $\frac{E^{2}}{I^{2}}$ according to equation (9) of Art. II6. This gives $R_{w} R_{l}$ $=\frac{E^{2}}{I^{2}}=\frac{L}{C}$, so that the condition of distortionless transmission is:

$$
R_{w} R_{l}=\frac{L}{C}
$$

where $R_{w}$ is the resistance of the wire* in unit length of the line, $R_{l}$ is the leakage resistance of unit length of the line, $L$ is the inductance per unit length of line and $C$ is the capacity per unit length of line.

123. Reduction of wire loss of energy by loading a line.A telephone line of which the inductance is increased by the insertion of coils of wire at intervals along the line as indicated in Fig. 162 is called a loaded line. The presence of these coils increases the resistance of the line slightly and also its capacity.

* Including both wires. 
In the following discussion this increase of capacity and resistance is ignored. The effect of loading a line (increase of $L$ ) is to decrease the value of the current for a given value of voltage in a wave, according to equation (9) of Art. II6, and therefore the rate of loss of energy because of wire resistance is decreased. A clear idea of the effect of loading may be obtained by considering a particular example:

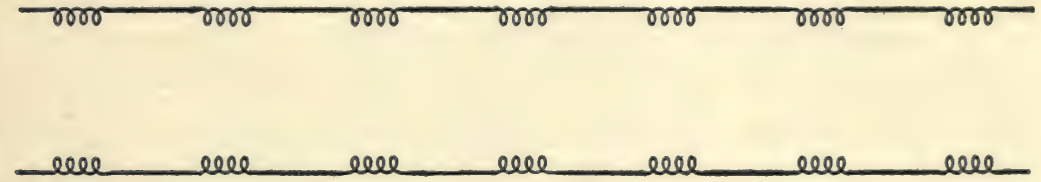

Fig. 162.

I. A battery of voltage $E$ is connected across the end of a line for one one-hundred-and-eighty-six-thousandth of a second, thus producing a rectangular wave pulse one mile long.

2. The total energy of the wave is $\frac{1}{2} L I^{2}+\frac{1}{2} C E^{2}$.

3. The rate of loss of energy in the wires is $R_{w} I^{2}$ watts.

4 The rate of loss of energy in the insulation is $\left(E^{2} / R_{l}\right)$ watts.

5. The time of transit of the wave from end to end of line is, say, $t$ seconds. Therefore:

6. The actual loss of energy in the wires is $R_{w} I^{2} t$ joules.

7. The actual loss of energy in the insulation is $\left(E^{2} / R_{l}\right) t$ joules.

Suppose now that $L$ is quadrupled by loading. Then the wave velocity is halved according to equation (8) of Art. II6 and the current in the wave is halved according to equation (9) of Art. I16, voltage in wave being unchanged. The following statements are therefore easily seen to be true:

I. The battery will have to be connected for two one-hundredand-eighty-six-thousandths of a second to give a wave one mile long, and, supposing this to be done, then:

2. The total energy of the wave is the same as before.

3. The rate of loss* of energy in the wires is one quarter as great as before. 
4. The rate of loss* of energy in the insulation is the same as before.

5. The time of transit of the wave is doubled. Therefore

6. The actual loss* of energy in the wires is half as much as before.

7. The actual loss* of energy in the insulation is twice as much as before.

Therefore, to quadruple the inductance of a line halves the wire loss of energy and doubles the leakage loss of energy for the same amount of energy transmitted.

The practical importance of loading of telephone lines grows out of the fact that in any well built line the insulation is very good so that the wire loss of energy is very much greater than the leakage loss, and the loading of a line produces a two-fold improvement, namely, $(a)$ the actual total loss of energy is reduced and $(b)$ wave distortion is greatly reduced by bringing wire loss of energy and leakage loss of energy more nearly into equality with each other.

Remark.-Pure wave transmission is markedly different from ordinary power transmission in that there is a fixed relation between voltage and current in a pure wave as explained in Art. II6, and a more efficient transmission cannot be obtained by increasing the voltage.

124. Electrical oscillation of a transmission line.-If a wave (like Fig. 146, for example) is set up on a transmission line it is repeatedly reflected at the ends of the line and made to travel back and forth over the line, and the line is said to oscillate. (a) If both ends of the line are open, the wave is reflected from one end with reversal of current, and again reflected from the other end with reversal of current, and thus the wave is brought back to its initial condition. Therefore the line passes through

* These energy losses are what are called first order approximations, inasmuch as they are calculated on the assumption that $E$ and $I$ in the wave keep their initial values during the entire transit of the wave from end to end of the line. 
one complete cycle of electrical "movement" during the time required for the wave to travel over twice the length of the line.

(b) If the line is short-circuited at both ends, the wave is reflected from one end with reversal of voltage, and again reflected from the other end with reversal of voltage, and thus the wave is brought back to its initial condition. Therefore the line passes through one complete cycle of electrical movement during the time required for the wave to travel over twice the length of the line.

(c) If the line is open at end $A$ and short-circuited at end $B$, the wave is reflected from end $A$ with reversal of current, then reflected from end $B$ with reversal of voltage, then reflected from end $A$ with reversal of current, and then reflected from end $B$ with reversal of voltage; and thus the wave is brought back to its initial condition. Therefore the line passes through one complete cycle of electrical movement during the time required for the wave to travel over four times the length of the line.

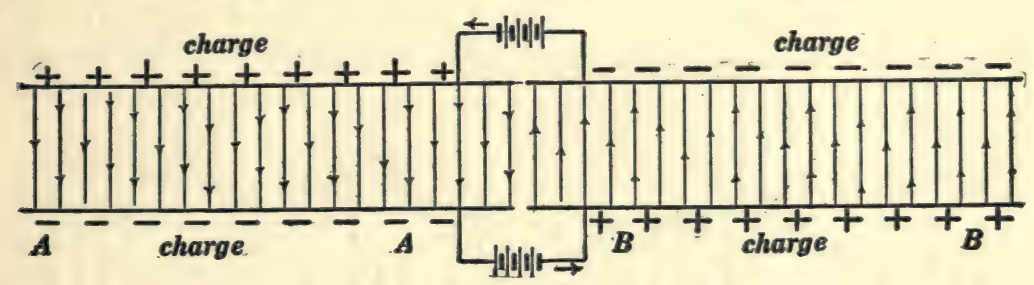

Fig. 163.

The behavior of a transmission line as described in connection with Figs. I53, I54 and I55 furnishes several good examples of transmission line oscillation. Another interesting example is as follows: Figure 163 shows a transmission line open at both ends, cut apart in the middle, and connected to two batteries as shown. One half of the line $A A$ is thus charged so as to give a downward electric field between the wires, and the other half $B B$ is charged so as to give an upward electric field between the wires as shown by the fine vertical lines which represent the lines of force of the electric field. When the two halves of the line $A A$ 
and $B B$ are suddenly connected the line oscillates electrically, * and Figs. I64, I65, I66 and 167 show four successive stages of one half of a complete oscillation. The electrical behavior of

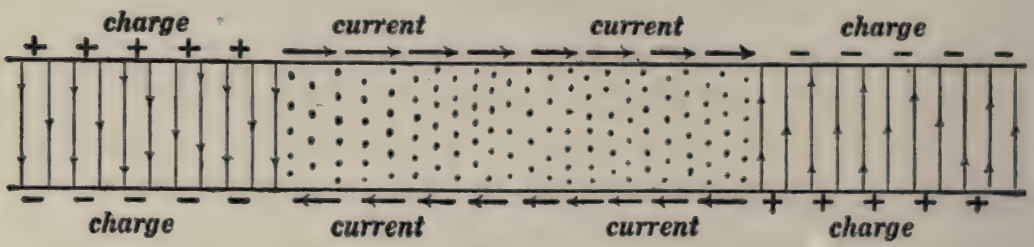

Fig. 164.

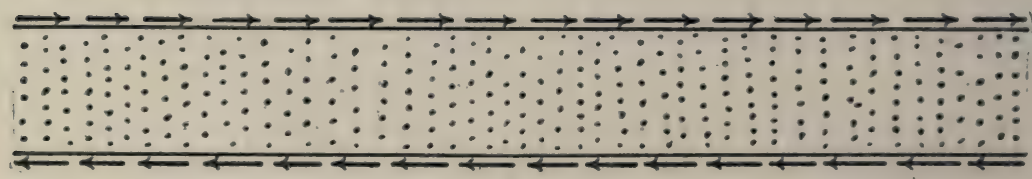

Fig. 165.

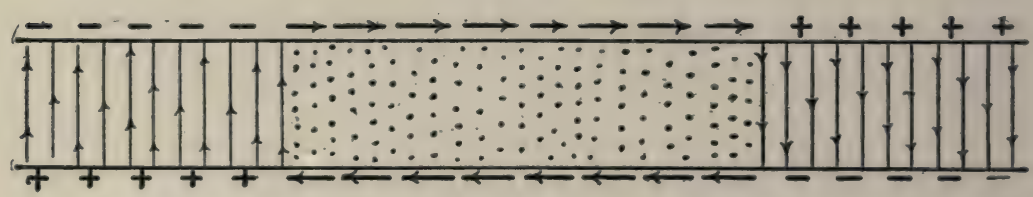

Fig. 166.

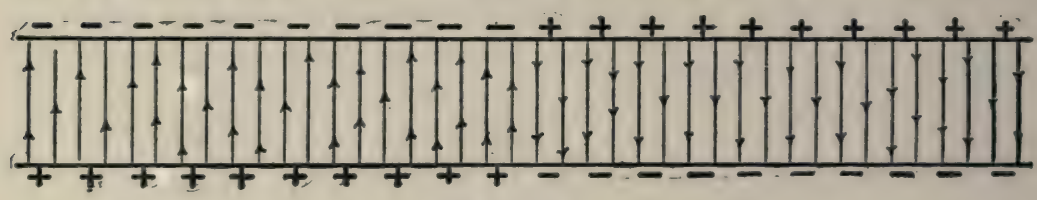

Fig. 167.

the line is completely represented in Fig. I68. When the two halves of the line in Fig. I63 are suddenly connected, a ribbon wave $F F$ shoots to the right from the middle point, and a ribbon wave $D D$ shoots to the left from the middle point; and these ribbon waves are repeatedly reflected or turned back at the open ends of the line, as indicated in Fig. 168. To find voltage distribution over the line at any instant the initial voltage dis-

* This particular case is mathematically identical to the vibration of a guitar string when it is plucked at its middle point. 
tribution as shown in Fig. 163 must be added to the sum of the voltages in the several laps of the two ribbon waves, and to find the current distribution over the line at any instant the currents in the several laps of the ribbon waves must be added.

wire

wire

wire

wire

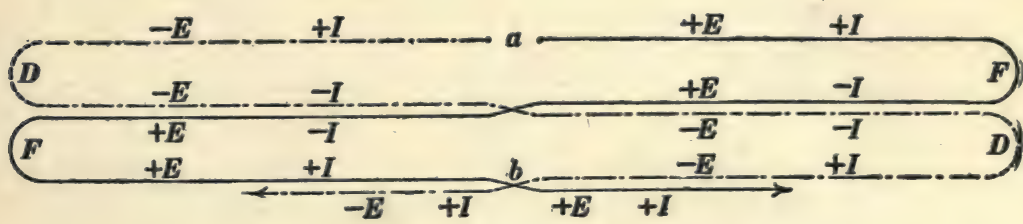

Fig. 168.

125. Moving wave trains.-Before considering the type of transmission line oscillation which is produced when an alternator is connected across one end of a line it is necessary to discuss more at length the type of wave train which is represented in Fig. I44. When such a wave train travels along a transmission line the voltage across the line at any point is a harmonic alternating voltage and the current in the line at any point is a harmonic alternating current of the same frequency, current and voltage are in phase with each other (line resistance and line leakage being negligible), and $\frac{E}{I}=\sqrt{\frac{L}{C}}$ where $E$ is the maximum value of the harmonic voltage, $I$ is the maximum value of the harmonic current, $L$ is the inductance of the line per unit length and $C$ is the capacity of the line per unit length.

Clock diagram models of moving wave trains.*-The parallel lines in the upper part of Fig. 169 represent a portion of a transmission line; $A B$ represents a long cylinder of wood with equi-

* The student is supposed to be familiar with the use of the so-called clock diagram in elementary alternating current theory. See Franklin and Esty's Elements of Electrical Engineering, Vol. II, pages 52-65. 
distant radial rods (arrows) set into it in a helical row; the cylinder with its attached arrows is supposed to be rotating at a speed of $f$ revolutions ( $=2 \pi f$ radians) per second in a counter-clockwise direction as seen from the end $B$; and the projections of the attached arrows upon the plane of the paper represent the instantaneous values at the various points of the transmission line of the alternating current $i$ which is associated with a simple wave train of electromagnetic waves moving along the transmission

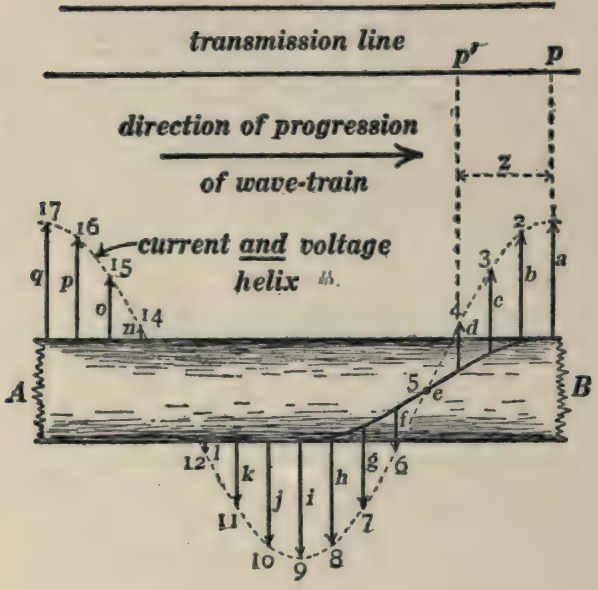

side view of clock-diagram model

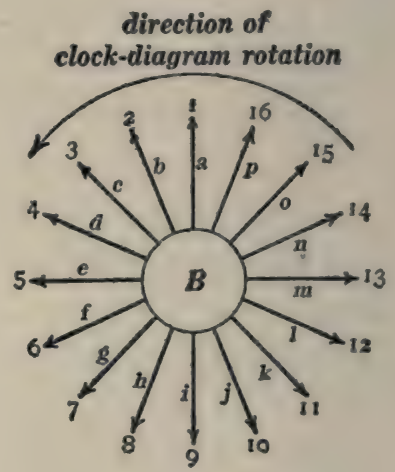

end view of model

Fig. 169.

Clock diagram model of a simple wave train moving to the right.

line from left to right. In a moving wave train, current $i$ and voltage $e$ are in phase with each other everywhere, so that the projections of the radial arrows in Fig. I69 represent the values of $e$ as well as the values of $i$.

We have chosen to consider both $i$ and $e$ positive (or both negative) in an electromagnetic wave moving to the right along the transmission line in Fig. 169. Therefore, in an electromagnetic wave moving to the left, the current and voltage must 
exrerywhere be opposite in sign, that is, wherever the one is positive the other must be negative. Thus the projections upon the plane of the paper of the numbered arrows in Fig. I 70 represent the instantaneous values at the various points of a transmission line of the alternating currents $i$ which are associated

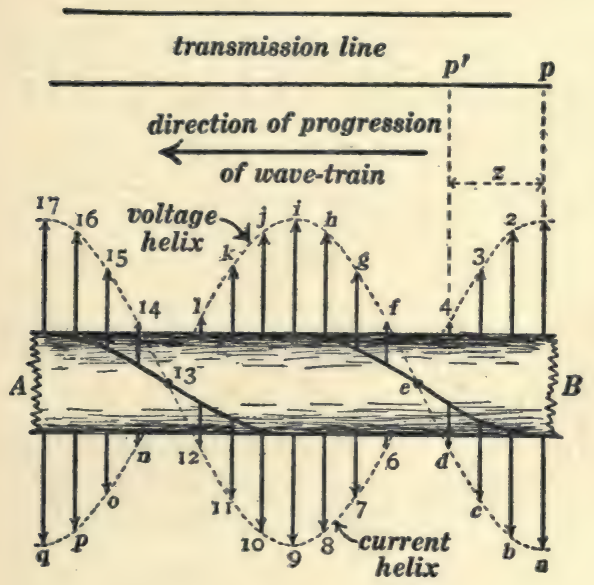

side view of clock-diagram model

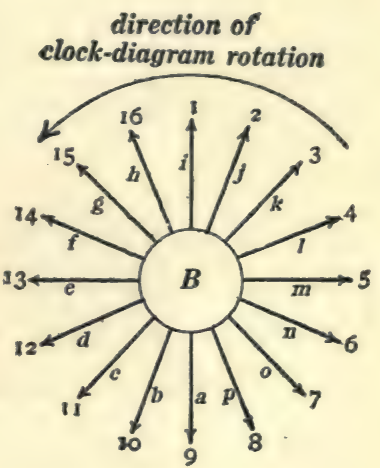

end view of model

Fig. 170.

Clock diagram model of a simple wave train moving to the left.

with a simple wave train moving from right to left, and the projections of the lettered arrows represent the instantaneous values of the alternating voltage $e$.

I26. Clock diagram model of a standing wave train.-When a string vibrates as a whole or in segments, the mode of motion of the string constitutes what is called a standing wave train, and in a simple standing wave train every particle of the string performs simple harmonic motion of the same frequency and of the same (or opposite) phase. The amplitude of the motion varies from zero at the nodes to a maximum at the antinodes. A clear idea of the mode of motion of such a string may be 
obtained with the help of Fig. I I in which $A \dot{B}$ is a wooden cylinder with equidistant radial rods (arrows) set into it in a straight row, that is, all lying in one plane; the wooden cylinder with its attached arrows is supposed to be rotating at a speed of

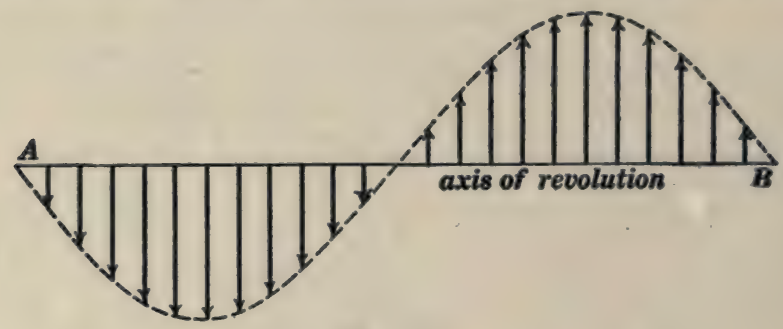

Fig. 171.

$f$ revolutions ( $=2 \pi f$ radians) per second in a counter-clockwise direction as seen from the end $B$; and the projections of the attached arrows upon the plane of the paper represent the up and down movements of the various particles of the vibrating string.

When a simple train of electromagnetic waves, like Fig. I44, travels over a transmission line and is reflected or turned back at the end of the line, the superposition of the original and reflected wave trains constitutes what is called a simple standing

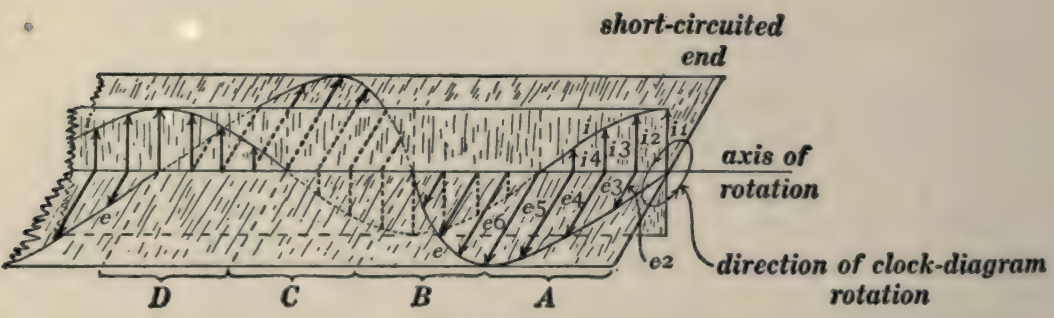

Fig. 172.

Clock diagram model of a standing wave train.

wave train or a simple mode of oscillation of the transmission line. A clear idea of voltage and current distribution over such an oscillating transmission line may be obtained with the help of Fig. 172. This figure represents two sets of equidistant radial 
rods (arrows), one set of rods (or arrows) being in one plane and the other set of rods (or arrows) being in a plane at right angles thereto; the whole is supposed to be rotating at a speed of $f$ revolutions per second in a counter-clockwise direction as seen from right hand end; and the projections of the arrows on the plane of the paper represent the instantaneous values of current and voltage at the various points of a transmission line which is oscillating in a simple mode or on which there is a standing wave train of electromagnetic waves.

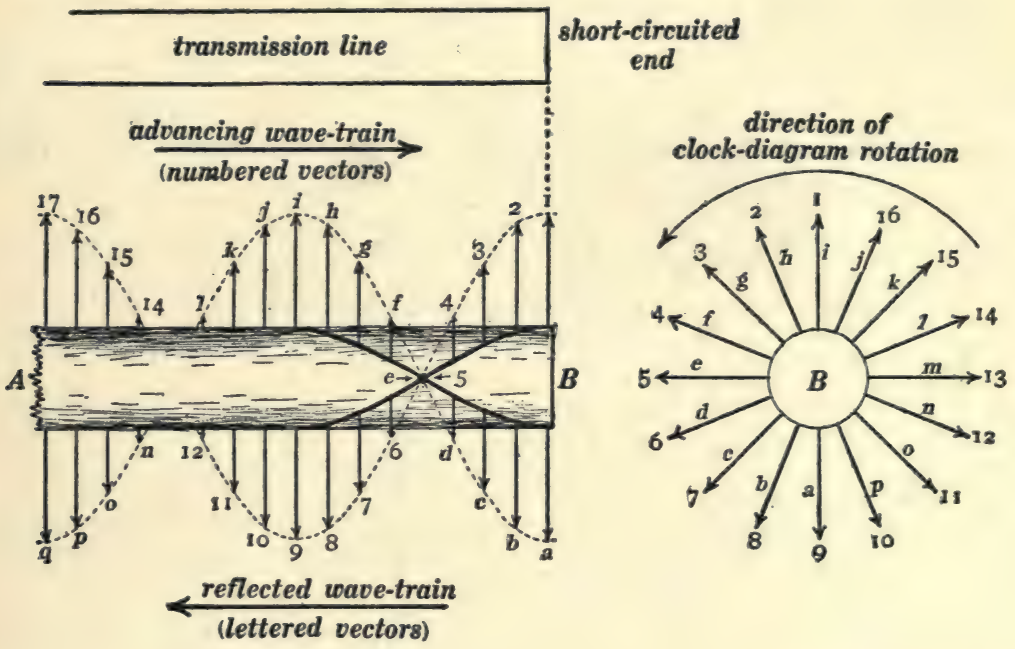

side view

end view

Fig. 173.

Clock diagram model of two simple wave trains, one moving to the right and one moving to the left.

The clock diagram model of Fig. I 72 may be derived from Figs. I69 and I70 by considering that a standing wave train is the superposition of two oppositely moving wave trains of equal amplitudes. Let us consider the voltage distribution in a standing wave train with the help of Fig. I73 in which the numbered arrows constitute the voltage helix from Fig. I69 (that is the numbered arrows in Fig. I73 represent the voltages along the 


\section{z36 ADVANCED ELECTRICITY AND MAGNETISM.}

line in a wave train moving to the right), and the lettered arrows constitute the voltage helix from Fig. I 70 (that is the lettered

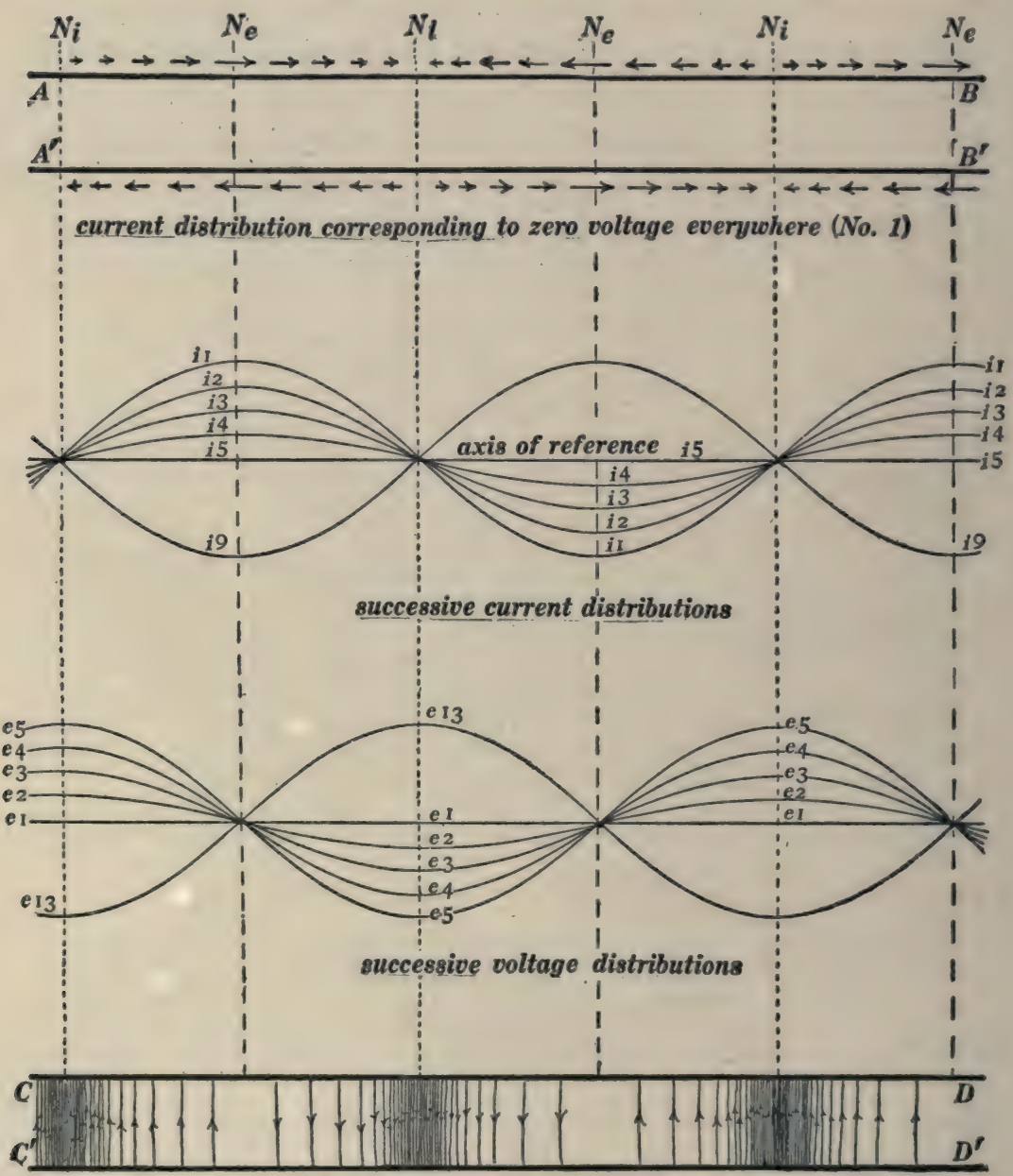

electric field (voltage) distribution corresponding to zero current everywhere (No.5)

Fig. 174.

arrows in Fig. $\mathrm{I} 73$ represent the voltages along the line in a wave train moving to the left). The actual voltage at each point in the standing wave train is the sum of the voltages at that point 
in the two oppositely moving wave trains, and it is represented therefore by a clock diagram arrow which is the vector sum of the clock diagram arrows which represent the voltages at that point in the respective moving trains. Now the vector sum of arrows $\mathrm{I}$ and $a$ in Fig. I 73 is evidently zero, the vector sum of arrows 2 and $b$ in Fig. 173 is the arrow $e_{2}$ in Fig. I72, the vector sum of arrows 3 and $c$ in Fig. 173 is the arrow $e 3$ in Fig. I72, and so on.

Let us now consider the current distribution in the standing wave train with the help of the current helices in Figs. I69 and I70. The vector sum of arrows No. I in Figs. I69 and I 70 is the arrow $i \mathrm{I}$ in Fig. I72, the vector sum of arrows No. 2 in Figs. $\mathrm{I} 69$ and $\mathrm{I} 7 \mathrm{O}$ is the arrow $i 2$ in Fig. I72, the vector sum of arrows No. 3 in Figs. 169 and $I 70$ is the arrow $i_{3}$ in Fig. I72, and so on.

The actual distribution of current and the actual distribution of voltage in a standing wave train on a transmission line is shown in Fig. I74. This figure represents several vibration segments in the middle portion of a long line. The upper part of the figure $A B A^{\prime} B^{\prime}$ represents the current distribution in the wires at the instant when the voltage between the wires is everywhere equal to zero, the flow of current at each point in the line being represented by the short arrows and also by the ordinate of the sine curve $i$. This current rapidly charges the wires (one wire positively and the other wire negatively) in the regions $N_{i}$, and as the charges increase the current values decrease. The ordinates of the successive curves $i_{1}, i_{2}, i_{3}$, $i_{4}$ and $i_{5}$, represent the successive values of the current at each point in the line, and the ordinates of the successive curves $e \mathrm{I}, e_{2}, e_{3}, e_{4}$ and $e_{5}$, represent successive values of the voltage across the line. The lower part of the figure, $C D C^{\prime} D^{\prime}$, represents the distribution of electric field (voltage between the wires) at the instant when the current is everywhere equal to zero.

The sine curves $i \mathrm{I}, i_{2}, i_{3}$, etc., and $e \mathrm{I}, e_{2}, e_{3}$, etc., in Fig. I74 represent the successive current and voltage distributions during one quarter of a cycle, that is, from the instant when 
voltage is everywhere zero to the instant when current is everywhere zero. At the instant which is represented by the curve e5 (when electric field is distributed as shown in the diagram

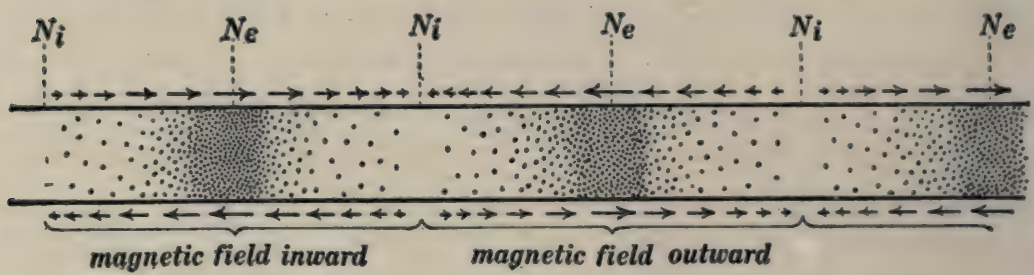

Fig. 175.

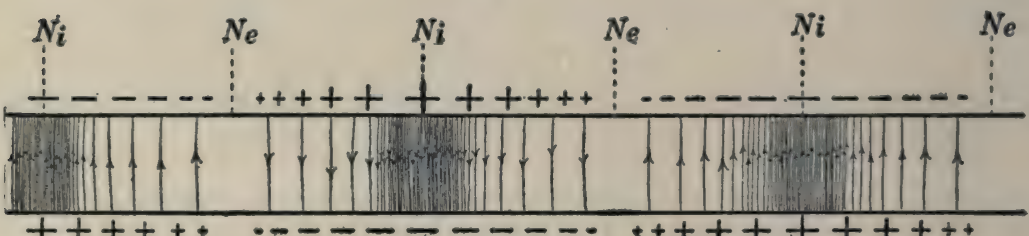

Fig. 176.

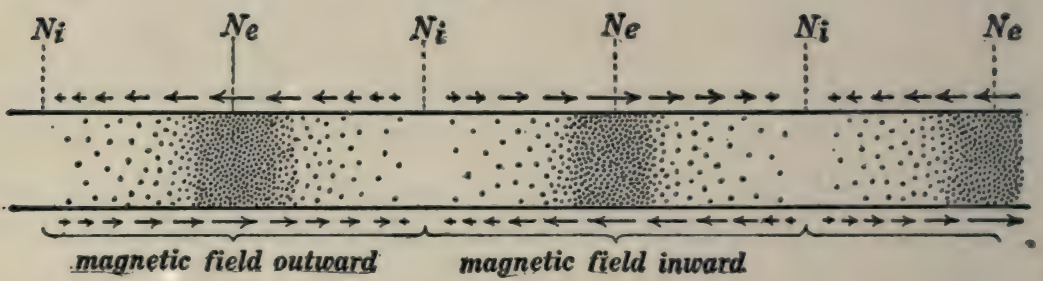

Fig. 177.

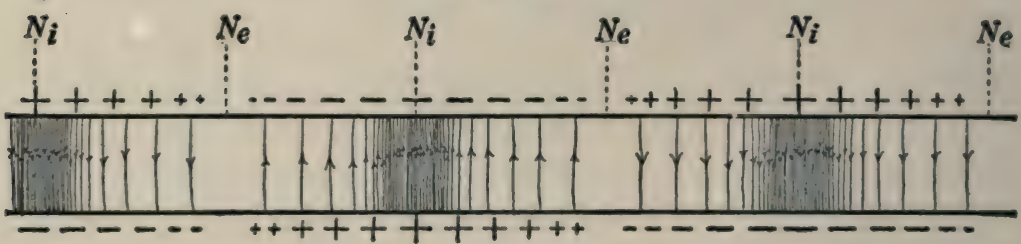

Fig. 178.

$\left.C D C^{\prime} D^{\prime}\right)$ current starts to flow at each point in the transmission line in directions opposite to the currents shown in the diagram $A B A^{\prime} B^{\prime}$, and these currents increase until they reach values represented by the ordinates of the curve is at which instant 
the voltage is again everywhere zero. These reversed currents then begin to produce reversed charges in the regions $N_{i}$, and after another quarter of a cycle these currents are reduced to zero and the voltage distribution over the line is represented by the ordinates of the curve $e \mathbf{I} 3$. The electric field distribution corresponding to the curve $e_{1} 3$ then establishes currents in the line and these currents grow to maximum values thus bringing the line back to initial condition $A B A^{\prime} B^{\prime}$ after one complete oscillation.

The actual distributions of current and magnetic field at the instants represented by curves $i_{\mathrm{I}}$ and $i_{9}$ are shown in Figs. I75 and 177 ; and the actual distributions of electric charge and electric field at the instants represented by curves $e_{5}$ and $e_{1} 3$ are shown in Figs. 176 and 178.

\section{voltage node}

voltage antinode
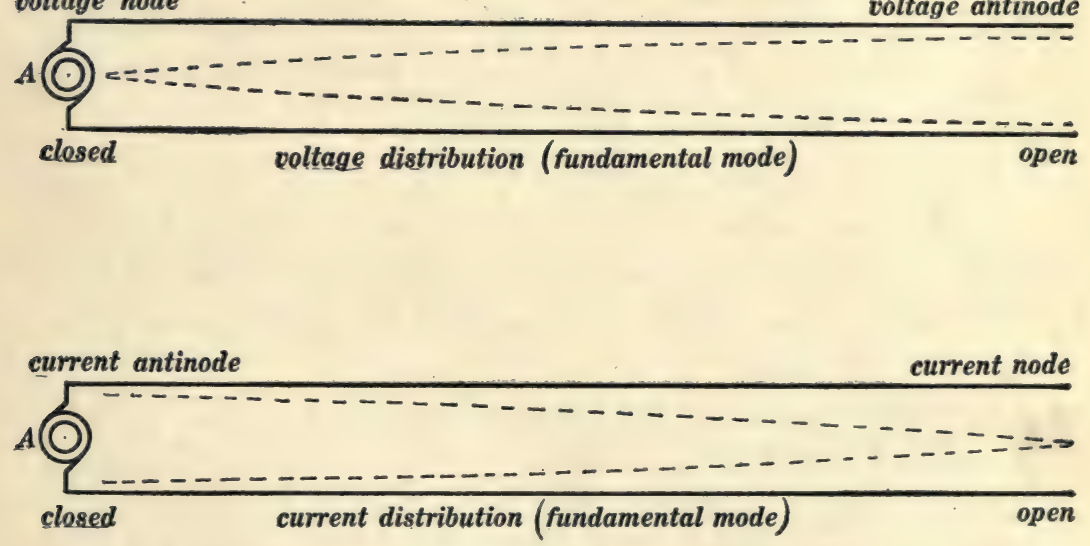

Fig. 179.

The portions of dotted sine curves in Fig. I 79 show the current and voltage distributions over a transmission line, open at the distant end, when the line is oscillating in its fundamental mode (lowest frequency), the oscillations being set up by the alternator $A$ of the proper frequency. When the current is everywhere zero, the voltages across the line are represented by the ordinates 
of one of the dotted curves in the upper half of the figure; and a quarter of a cycle later, when the voltage across the line is everywhere zero, the currents in the line are represented by the ordinates of one of the dotted curves in the lower half of the figure.
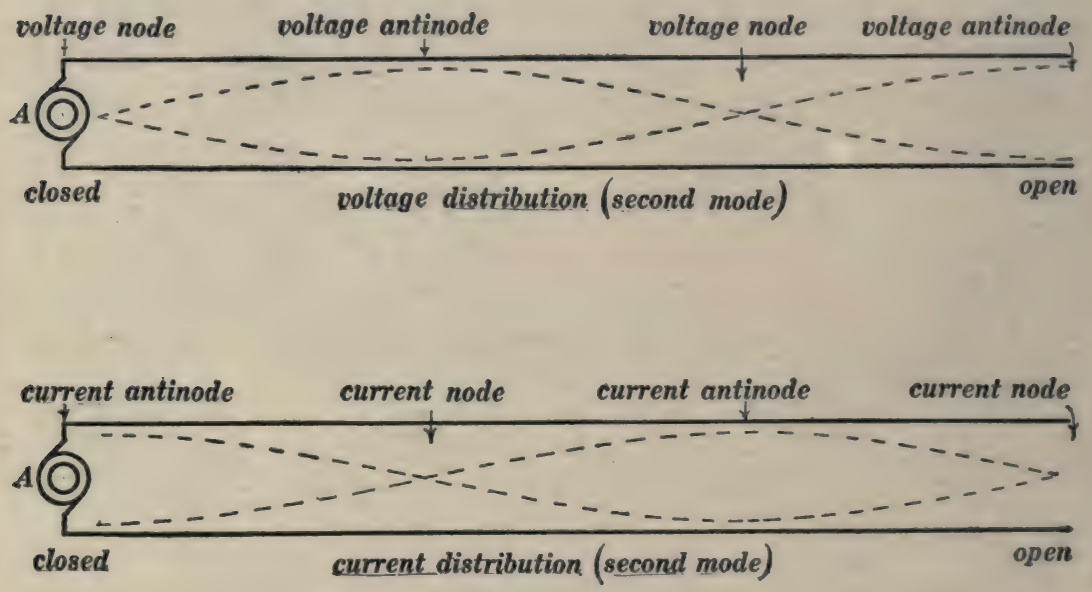

Fig. 180.

The dotted curves in Fig. I80 represent current and voltage distribution over a transmission line when it is oscillating in its second mode (next to lowest frequency), the oscillations being set up by the alternator $A$ of proper frequency.

The time of one complete oscillation of a transmission line is the time required for an electric wave to travel (at a speed of I86,000 miles per second) over one whole wave-length of the standing wave train, and the wave-length of a standing wave train is twice the length of one vibrating segment, from one current node to the next current node. Thus the whole length of the line in Fig. I 79 is one quarter of a wave-length of the standing wave train, the whole length of the line in Fig. I 80 is three quarters of a wave-length of the standing wave train.*

* The student should be able to make diagrams somewhat like Figs. I 79 and 180 but showing current and voltage distributions for the first and second modes of oscillation of a transmission line which is short-circuited at one end and connected 
127. Forced oscillations of a transmission line.-The alternator in Figs. I 79 and 180 is assumed to have a frequency equal to the frequency of free oscillation of the line, and the oscillations which are represented in Figs. I79 and I80 are called free oscillations. When the frequency of the alternator is not the same as the frequency of free oscillation of the line, then we have what is called a forced oscillation of the line, and the alternator is, in this case, not at a voltage node.

If the frequency of the alternator coincides with the frequency of one of the simple modes of oscillation of the transmission line, the violence of the oscillations will become very great if the line losses are small, in fact the violence of the ultimate oscillations which are built up in this case is limited only by the line losses.

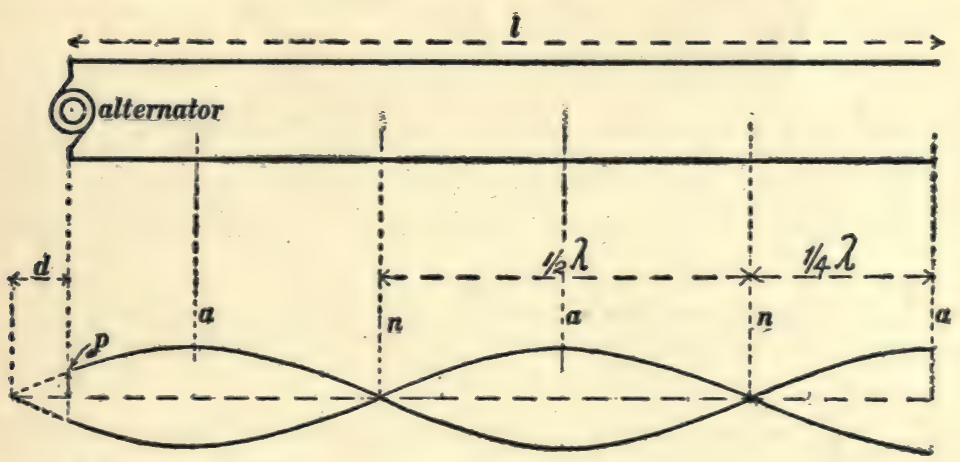

Fig. 181.

Voltage distribution over oscillating transmission line open at far end.

When the frequency of the electromotive force of the alternator does not coincide with one of the simple modes of oscillation of the line, the ultimate steady state of oscillation of the line is determined as follows (line losses being ignored). Figures I8I and 182 represent voltage distributions over a transmission line which is connected to an alternator, the distant end of the

to an alternator of the proper frequency at the other end. The alternator in Figs. 179 and $r 80$ is assumed to have a very low voltage because after the line is once oscillating (its resistance and leakage being assumed to be negligible) no perceptible generator voltage is required to maintain the oscillation. 
line being open; and Figs. 183 and 184 represent voltage distributions over a transmission line which is connected to an alternator, the distant end of the line being short-circuited.

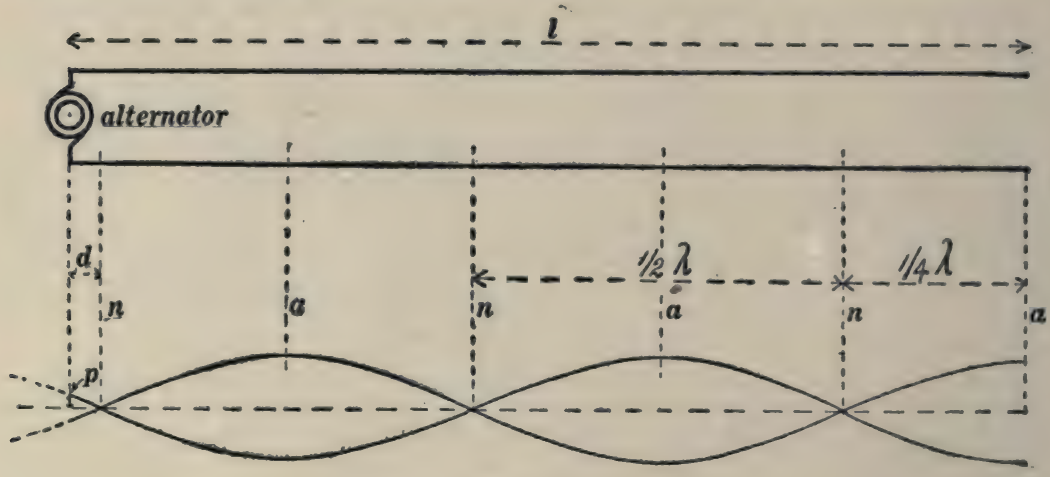

Fig. 182.

Voltage distribution over oscillating transmission line open at far end.

The action which takes place during the building up of the steady state of oscillation of the transmission line is rather complicated and will not be considered. The ultimate state of

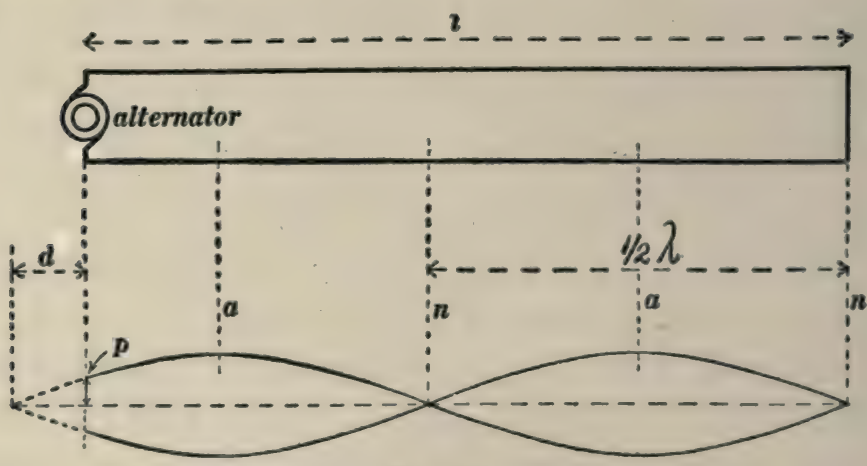

Fig. 183.

Voltage distribution over oscillating transmission line closed at far end.

steady oscillation of the line is a standing wave train of which the wave-length $\lambda$ is equal to the distance traveled by an electromagnetic wave during one complete cycle of the electromotive force of the alternator. For example, if the alternator 
has a frequency of 10,000 cycles per second, then the wavelength $\lambda$ will be 18.6 miles. The voltage nodes (current antinodes) of this standing wave train are at the points $n, n, n$,

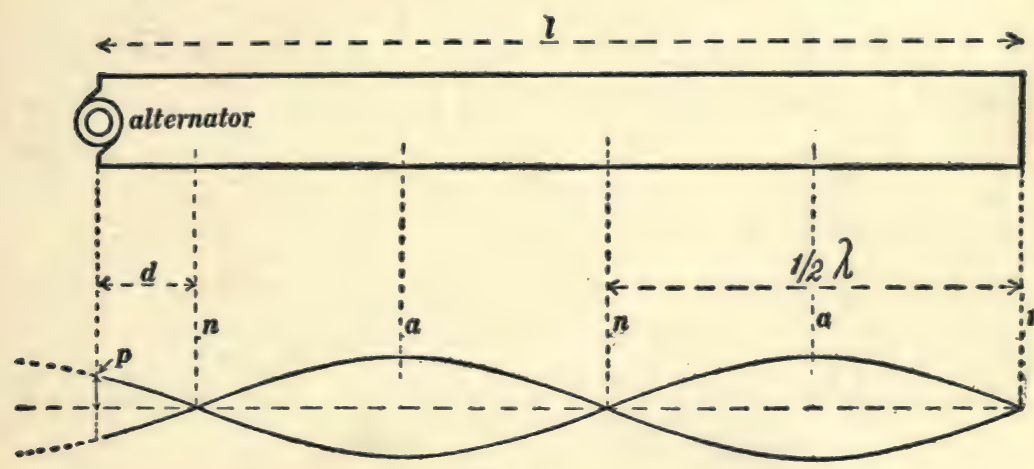

Fig. 184.

Voltage distribution over oscillating transmission line closed at far end.

etc., and the voltage antinodes (current nodes) are at the points $a, a, a$, etc. The voltage across the line at any point is an alternating voltage of which the frequency is equal to the frequency of the alternator, and the maximum value of the voltage across the line at any point is represented by the ordinate at that point of one of the sine curves in Figs. 18I to 184. Let $E$ be the maximum value of the voltage between wires at any voltage antinode. Then the maximum voltage between wires at a point distant $x$ from a voltage antinode is $E \sin \frac{2 \pi x}{\lambda}$. The condition which must be satisfied when the ultimate steady state of line oscillation is reached is that the distribution of voltage over the line as represented by the sine curves in Figs. I8I to I84 must conform to or fit the alternator voltage at the generator end. Therefore the ultimate state of line oscillation may be determined as follows: Begin at the distant end of the line. Lay off a number of quarter wave-lengths and determine the distance $d$ which is left over at the generator end of the line as represented in Figs. I8I to I84. Then the maximum voltage $E$ at one of the voltage 
antinodes and the maximum generator voltage $G$ must satisfy the equation:

$$
G=E \sin \frac{2 \pi d}{\lambda}
$$

128. Experimental demonstration of standing waves on a pair of straight wires. - The velocity of progression of an electromagnetic wave on a plain transmission line is the same as the velocity of light, namely, $\mathbf{1 8 6 , 0 0 0}$ miles per second, and therefore it is necessary to use an alternating electromotive force of extremely high frequency if one is to produce a standing wave train upon a plain transmission line of moderate length. The arrangement used by Lecher is shown in Fig. 185. Two flat plates of

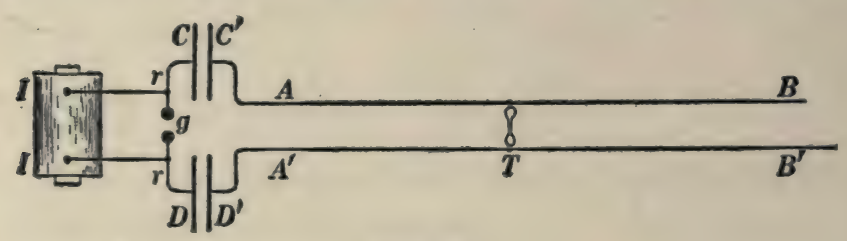

Fig. 185.

metal $C$ and $D$ together with the rods $r$ and the air gap $g$ constitute a Hertz oscillator which is excited from the induction coil $I I$; and $C^{\prime} D^{\prime}$ are two auxiliary metal plates which are connected to the long wires $A B A^{\prime} B^{\prime}$. A rapid reversal of electric charge on the plates $C$ and $D$ (produced by the electric oscillations of the system $C r g r D$ ) induces reversals of charge upon the plates $C^{\prime}$ and $D^{\prime}$, and the result is that a train of waves is sent out along the pair of wires and reflected from its distant end, thus producing a standing wave train. The voltage distribution over the transmission line $A B A^{\prime} B^{\prime}$ may be investigated by bridging a small vacuum tube across the line as shown at $T$ in Fig. 185 . When this vacuum tube is slid along the wires it shows no luminosity at or near the voltage nodes, whereas it shows a maximum luminosity at or near the antinodes. The best form of vacuum tube for this purpose is shown in Fig. 186, the narrow portion of the tube should have a bore of about one 
millimeter and it should be filled with the rare gas neon at a pressure of 2 or 3 millimeters of mercury.

The open end $B B^{\prime}$ of the "transmission line" in Fig. 185 is a voltage antinode, and usually a number of voltage nodes are found by moving the vacuum tube $T$ along. It is interesting to note in this connection that the frequency of the oscillations

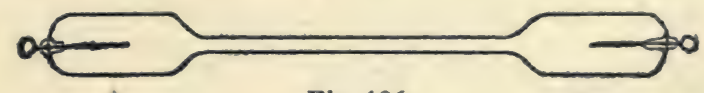

Fig. 186.

of the system $\operatorname{CrgrD}$ may be found by measuring the distance between adjacent voltage nodes on the "transmission line" $A B A^{\prime} B^{\prime}$, remembering that this is the distance travelled by an electromagnetic wave during one-half of a complete oscillation. Thus, if the distance between adjacent voltage nodes is 3 feet the total wave length is 6 feet, and the period of one oscillation is the time required for an electromagnetic wave to travel over this distance, namely 6.I $\times \mathrm{IO}^{-9}$ of a second, which corresponds to a frequency of $320,000,000$ oscillations per second.

\section{ELECTROMAGNETIC ACTION.}

\section{I29. Mechanical conceptions of magnetic and electric fields.*} -It is of the greatest help towards the clear understanding of electric and magnetic phenomena to obtain clear mechanical

* Sir Oliver Lodge's Modern Views of Electricity is perhaps the best elementary treatise on this subject.

The most complete mechanical conception of the electromagnetic field is that which is based upon Lord Kelvin's gyrostatic model of the ether. This gyrostatic model of the ether is a mechanical structure which is capable of reproducing most of the known phenomena of electricity and magnetism and of light. See Aether and Matter, by Joseph Larmor, Appendix E, Cambridge, I900. Lord Kelvin's gyrostatic model of the ether has led to a hydrodynamic conception of the ether, due chiefly to Larmor, in which the ether is assumed to be a perfect fluid endowed with the necessary elastic properties by an indefinitely fine-grained whirling motion. On the basis of Lord Kelvin's gyrostatic conception of the ether and also on the basis of Larmor's turbulent ether, the magnetic field is thought to consist of a simple flow of ether along the lines of force of the magnetic field. This conception of the magnetic field is very different from the conception (Maxwell's) which is outlined in this text. 
ideas of electromagnetic action. Maxwell was the first to work out such mechanical ideas, and Maxwell's conceptions are made use of in the following discussion. Everything in this discussion refers strictly to two dimensions; certain inconsistencies arise in the attempt to extend these conceptions to three dimensions.

Maxwell's model of the ether.-The ether is to be considered as built up of very small cells of two kinds, positive cells and

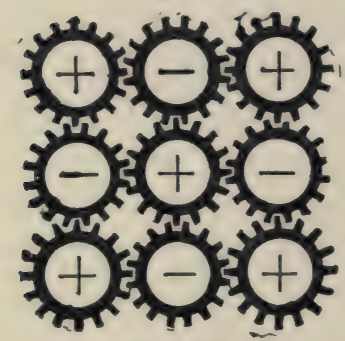

-. Fig. 187. negative cells, in such a way that only unlike cells are in contact." These cells are imagined to be gear wheels provided with rubber-like teeth so that if a cell be turned while the adjacent cells are kept stationary a torque due to the elastic distortion of the gear teeth is brought to bear upon the turned cell. Figure I87 shows five positive cells and four negative cells geared together in the manner specified. In subsequent figures, these cells or cog-wheels are represented by plain circles for the sake of simplicity.

Conception of the magnetic field.-The ether cells in a magnetic field are thought of as rotating about axes which are parallel to the direction of the magnetic field at the point, the angular velocity of the cells being proportional to the intensity of the field. The positive cells rotate in the direction in which a righthanded screw would be turned that it might travel in the direction of the field, and the negative cells rotate in the oppo-

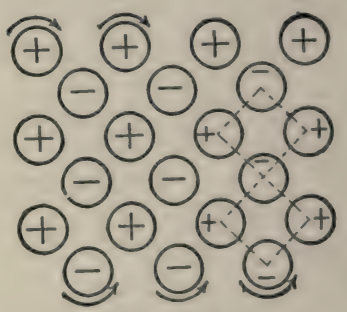

Fig. 188. site direction. This opposite rotation of positive and negative cells is mechanically possible because unlike cells only are geared together. Thus, in Fig. I88, the positive cells are represented as rotating in a clock-wise direction, and the negative cells as rotating in a counter-clockwise direction; this is a state of motion 
which represents a magnetic field perpendicular to the plane of the paper and directed away from the reader. The energy of the magnetic field is represented by the kinetic energy of rotation of the ether cells.

Conception of the electric field.-The positive ether cells in an electric field are thought of as being displaced in the direction of the field while the negative cells are displaced in the opposite direction, and this displacement is assumed to be proportional to the electric field intensity. Thus, Fig. 189 represents the positive cells as being displaced towards the bottom of the page relatively to the negative cells, as shown by the arrows, that is to say, the distortion of the ether struc-

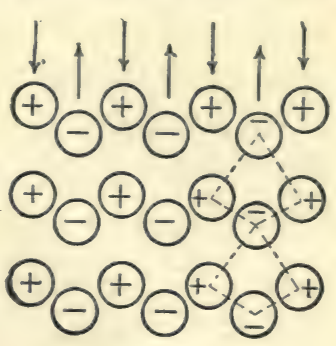

Fig. 189. ture which is shown in Fig. I89 represents an electric field directed towards the bottom of the page. Figure I90 shows two

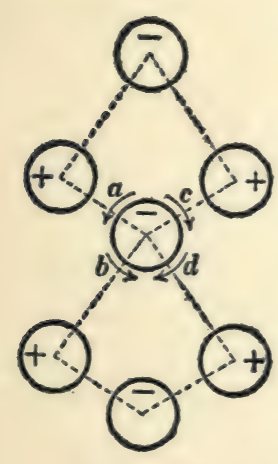

Fig. 190. meshes of the cellular structure of the distorted ether of Fig. I89. These two meshes are square in the undistorted ether as shown in Fig. 188, whereas the downward displacement of the positive cells in Fig. I89 has distorted these meshes as shown in Figs. I89 and 190. Inasmuch as the cell structure of the ether is assumed to be elastic (the gear teeth in Fig. I 87 being made of substance like rubber), the distortion of the ether structure which is shown in Fig. I 89 represents potential energy, and this energy is the energy of the electric field.

An understanding of the magnetic action of what may be called a tapering electric field may be arrived at by considering the torque action which is exerted upon a given cell by the elastic distortion which is represented in Figs. I89 and 190. Consider the two 
positive cells to the left of the middle cell in Fig. I90; these two positive cells have been displaced downwards with respect to the middle cell, and they exert torques upon the middle cell which are represented by the small curved arrows $a$ and $b$. Consider the two positive cells to the right of the middle cell in Fig. 190; these two positive cells have been displaced downwards with respect to the middle cell, and they exert torques upon the middle cell which are represented by the small curved arrows $c$ and $d$. If the electric field is uniform (same value on both sides of the middle cell in Fig. 190), then the torques $a$ and $b$ balance the torques $c$ and $d$, and the distortion which is represented in Fig. I89 has no influence upon the rotatory motion of the cells.

I30. Magnetic action of a tapering electric field.-Imagine an electric field of which the lines of force are straight, as shown in Fig. I9I, but of which the intensity falls off as shown, that is to

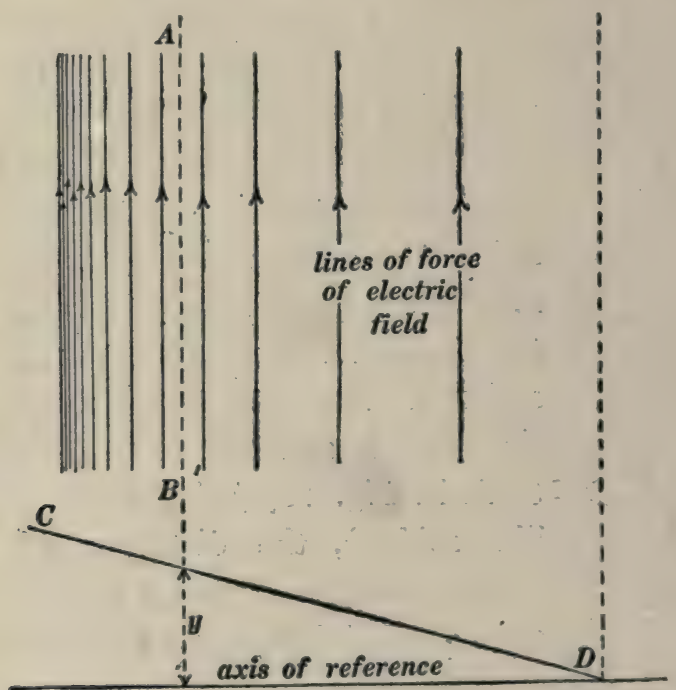

Fig. 191.

say, the intensity of the electric field at any place along the line $A B$ in Fig. I9I is represented by the ordinate $y$ of an inclined straight line $C D$. Such an electric field is called a tapering 
fiuld for the sake of brevity. Imagine the ether cells in Fig. 189 to be displaced in a manner to represent the tapering electric field of Fig. I9I. Then the two positive cells to the left of the middle cell in Fig. I90 are displaced downwards more than the two positive cells to the right of the middle cell in Fig. 190, so that the two torques $a$ and $b$ exceed the two torques $c$ and $d$, that is, the middle cell is acted upon by an unbalanced torque, and, therefore, the middle cell must be gaining angular velocity about an axis perpendicular to the plane of the paper. What is here said of this particular cell is true of all the ether cells in Fig. I89 in the case of a tapering electric field; therefore, $a$ tapering electric field produces a continuously increasing magnetic field at right angles to itself.*

I3I. Electric action of a tapering magnetic field.-Hereafter a chain of geared cells which is free from distortion will be thought of as standing in a straight row as shown in Fig. I92a,

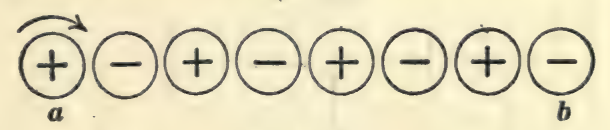

Fig. 192a.

and the opposite displacements of positive and negative cells which constitute an electric field will be thought of as changing such a straight row to a zigzag row, as shown in Fig. 192b. Consider a number of geared cells which tend by elastic action of any kind to stand in a straight row like Fig. 192a. Such a row is converted into a zigzag row as shown in Fig. $192 b$ if the cell $a$ is turned while the cell $b$ is kept stationary.

* This is a modified form of statement of the law of induced electromotive force. It is a verbal statement of the differential equation

$$
\frac{d M}{d t}=A \frac{d Z}{d x}
$$

in which $M$ is the $y$-component of magnetic field, and $Z$ is the $z$-component of electric field. One form of this differential equation is derived and discussed in Art. II6. 


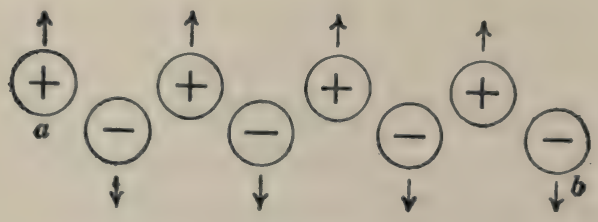

Fig. $192 b$.

Figure 193 represents a tapering magnetic field the intensity of which at any place along the line $A B$ is represented by the ordinate $y$ of the inclined straight line $C D$. Looking endwise at the lines of force of the magnetic field one may, as it were,

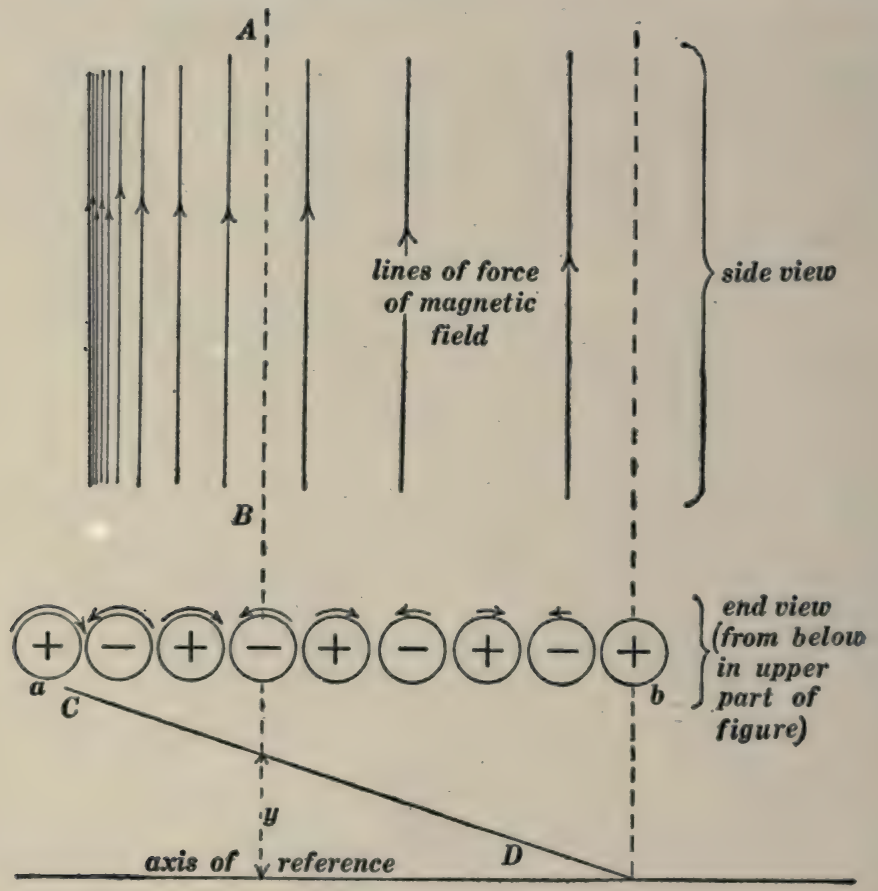

Fig. 193.

see the ether cells rotating as shown by the curved arrows in the end view, Fig. 193, cell $a$ rotating at high speed and cell $b$ being stationary. The result of this difference of speed is to 
carıse a continually increasing zigzag distortion of the chain of ether cells $a b$, and this zigzag distortion constitutes an electric field at right angles to the magnetic field in Fig. 193, that is to say, a tapering magnetic field produces a continuously increasing electric field at right angles to itself.*

I32. The electric wave.-Figure I94 represents an electric wave traveling along a transmission linet in the direction of the

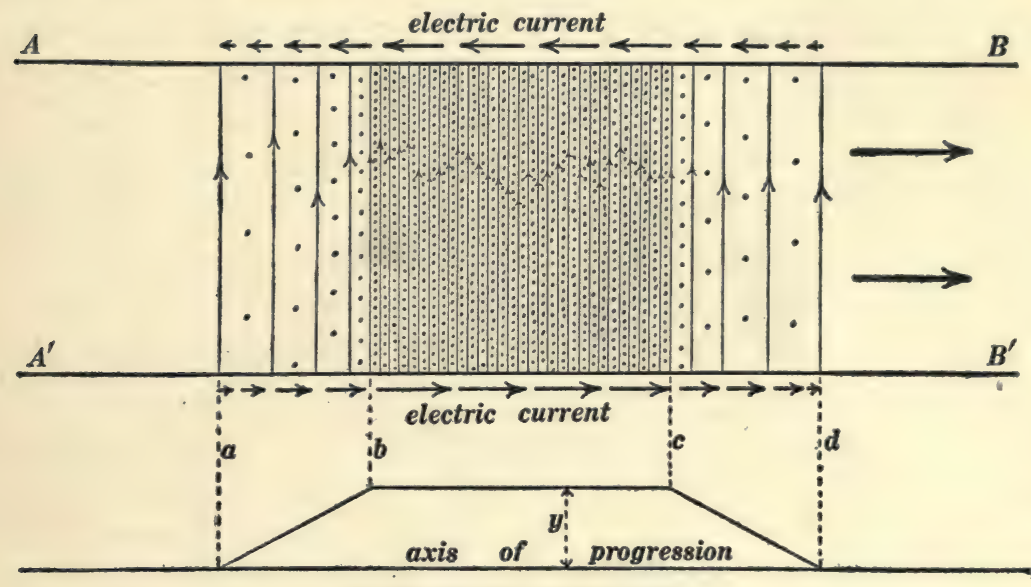

Fig. 194.

heavy arrows. The fine vertical lines represent the lines of force of the electric field and the dots represent the lines of force of the magnetic field which is perpendicular to the plane of the paper. The tapering electric field in the front part of the wave causes a continual increase or building up of magnetic field in that part of the wave, the tapering magnetic field in the front part

* This is a modified form of statement of the law of induced magnetomotive force. It is a verbal statement of the differential equation

$$
\frac{d Y}{d t}=A \frac{d N}{d x}
$$

in which $\boldsymbol{Y}$ is the $y$-component of electric field and $N$ is the $z$-component of magnetic field. One form of this differential equation is discussed in Art. I 6 .

† The wires in Fig. 194 are supposed to be broad flat metal ribbons. 
of the wave causes a continual building up of the electric field in that part of the wave, the tapering electric field in the back part of the wave causes a continual decrease or letting down of the magnetic field in that part of the wave, and the tapering magnetic field in the back part of the wave causes a continual letting down of the electric field in that part of the wave. Thus the wave is being continually let down in the rear and built up in front and energy is continually streaming from the back part of the wave where the wave is being let down to the front part where the wave is being built up.

133. The electric current.-Consider a wire* $A B$, Fig. I95, along which an electric current is flowing steadily from $B$ towards $A$. The magnetic field on opposite sides of $A B$ is in opposite directions, so that the positive ether cells at $p$ and $p^{\prime}$ are rotating in opposite directions as indicated. A steady

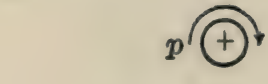

A

\section{B}

car
cannot be accommodated by

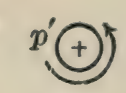

Fig. 195. electric current may be maintained for an indefinite length of time, but the opposite rotations of positive ether cells on the two sides of $A B$, Fig. I95, an ever-increasing ether distortion (distortion of the rubber-like teeth of the ether cells as shown in Fig. 189), there must be a slip between adjacent cells somewhere between $p$ and $p^{\prime}$. This slip between adjacent ether cells takes place in the material of the wire and constitutes the electric current.

Steady electric currents flow in closed circuits.-Maxwell's conception of the ether shows that this must be true as follows: Consider the opposite rotations of like ether cells at $p$ and $p^{\prime}$, Fig. 195, and consider a chain of geared cells passing from $p$

* Strictly, a broad metal sheet. Maxwell's conceptions apply to two dimensions only. 
to $p^{\prime}$ around the end $A B$ as shown by the curved line in Fig. 196. The current in $A B$ is assumed to be maintained indefinitely, and therefore the opposite rotation of $p$ and $p^{\prime}$ is assumed to continue indefinitely, but since this continued opposite rotation of $p$ and $p^{\prime}$ cannot be accommodated by everincreasing distortion of the elastic gear teeth of the ether cells along the chain which passes around the end of $A B$, a slip must take place between adjacent cells at some point along this chain. There-

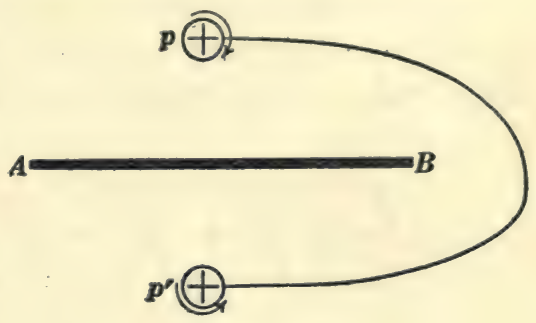

Fig. 196. fore the line of flow of the current $A B$ (line of slip of geared cells) must form a closed circuit which cuts across every possible chain of geared cells extending from $p$ to $p^{\prime}$. When a current flows along a path which does not form a closed circuit, then an increasing ether distortion (electric field) is produced around the end portions of the path as explained in Art. I35.

\section{r34. The flow of energy in the electromagnetic field. Poyn-} ting's theorem.-It has been shown by J. H. Poynting* from theoretical considerations that energy streams through an electromagnetic field in a direction at right angles both to the electric field and to the magnetic field at each point, and that the amount of energy per second which streams across one square centimeter of area is proportional to the product of the electric and magnetic field intensities. If the electric and magnetic fields are not at right angles to each other, the energy stream is proportional to the product of the intensities of the two fields and the sine of the included angle.

Conception of the energy stream.-Consider a row of gear wheels, as shown in Fig. 197. Imagine the wheel $W$ to be turned

* See Philosophical Transactions, Vol. I75, Part II, page 343, I884. This original paper by Poynting is well worth reading. 
steadily by a crank, and the wheel $W^{\prime}$ to be hindered by a brake. Under these conditions energy would be continuously transmitted along the chain of gear wheels from $W$ to $W^{\prime}$. Each wheel of the chain would be acted upon by equal and opposite torques by the wheels on either side of it, the transmission of energy along the chain would depend upon this torque action and the rotatory motion of the wheels, and the rate at which energy would

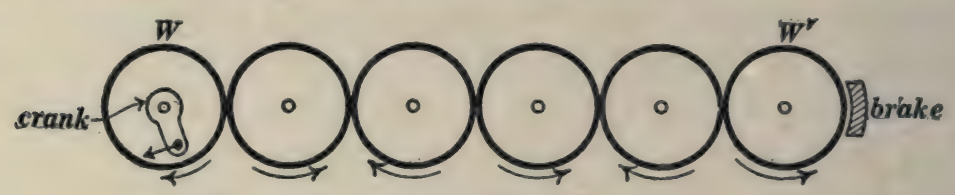

Fig. 197.

be transmitted along the chain would be proportional to the product of the speed of the wheels and the torque action between adjacent wheels.

Imagine the ether cells in Fig. I 89 to be rotating, positive cells in one direction and negative cells in the other direction, about axes perpendicular to the plane of the paper. This rotatory motion constitutes a magnetic field perpendicular to the plane of the paper and perpendicular to the electric field which is towards the bottom of the page in Fig. I89. On account of the torque action between the cells (as explained in connection with Fig. 190) combined with the rotation of the cells, energy is transferred to the right (or left) by each horizontal chain of geared cells in Fig. 189 at a rate which is proportional to the product of the intensity of the magnetic field and the intensity of the electric field; and the energy per second flowing across an area (at right angles to both electric and magnetic fields) is proportional to the product of the respective field intensities and proportional to the area, inasmuch as the area determines the number of rows of cells which participate in the transfer of the energy.

Examples of Poynting's theorem. (a) The flow of energy in the neighborhood of a wire carrying an electric current when no electric charge resides on the surface of the wire.-In general, the 
surface of a wire which carries an electric current is charged,* and this charge is associated with the lines of force of electric field which is perpendicular to the surface of the wire. There is always a point on any wire circuit, however, where the wire is not charged or, in other words, where the electric field is parallel to the surface of the wire. In any case, however, it is permissible to consider that part of the energy flow which depends upon the component of the electric field parallel to the wire. Figure 198 shows a straight wire carrying electric current. The lines of force of the electric field are parallel to the-wire as shown in the side view, and the lines of force of the magnetic field encircle the wire as shown in the end view. The dotted lines represent the energy stream which flows in towards the wire from all sides.

The equation for the energy stream in an electromagnetic field may be established by considering the inward flow of energy in the neighborhood of a wire carrying an electric current as follows: Let $R$ be the resistance of the wire in abohms per

* The component of the electric field which is parallel to the surface of a wire is always equal to $R I$, where $R$ is the resistance of the wire per centimeter of length, and $I$ is the current flowing in the wire; but the component of the electric field at right angles to the surface of the wire may have any value whatever. The electric lines of force which terminate on the surface of the wire on account of the existence of this normal component of the electric field involve a stationary electric charge on the surface of the wire. The electric current is of course considered to be a transfer of electric charge along a wire, but the stationary charge here referred to has nothing directly to do with the current. When a voltaic cell is on open circuit, the electric field in the surrounding region may be such that the volts per centimeter along a given path may vary in the most irregular way; but when this path is occupied by a wire through which the voltaic cell produces current, then the electric field in the whole surrounding region is modified by the stationary charge on the surface of the wire so as to make the component of the electric field parallel to the wire everywhere equal to $R I$, as above specified. Energy appears in each unit length of the wire at the rate of $R I^{2}$ ergs per second. This amount of energy must flow into every unit length of the wire, and the electric field in the neighborhood of the wire must be so distributed as to give this necessary distribution of the energy stream. It is to be remembered that the trend of the magnetic field in the neighborhood of an electric circuit depends only on the shape of the circuit but not at all on the relative resistances of the various parts of the circuit, and, therefore, the proper distribution of the energy stream to supply the $R I^{2}$ losses at each part of a circuit depends, one might say, chiefly upon the modification of the electric field due to surface charges on the wire. 
centimeter of length, and let $I$ be the current in the wire in abamperes, then $R I$ is the intensity of the surrounding electric field in abvolts per centimeter. The intensity of the magnetic field at a distance of $r$ centimeters from the axis of the wire is $2 I / r$ gausses.* The intensity of the energy stream (ergs of

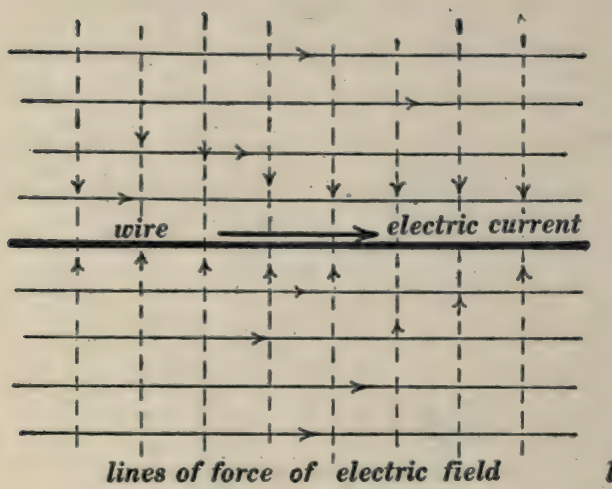

side view

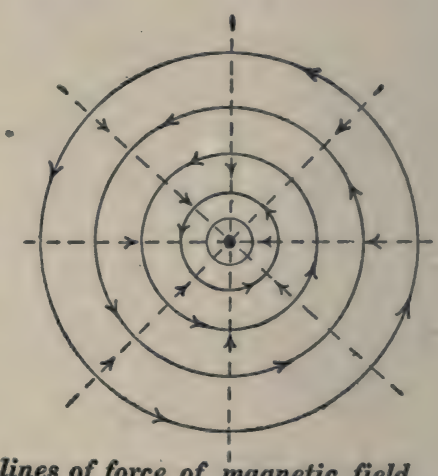

end view

Fig. 198.

energy per second per square centimeter) at points distant $r$ centimeters from the axis of the wire is proportional to the product of the electric and the magnetic field intensities, and it may therefore be written $k \times R I \times 2 I / r$, where $k$ is an unknown proportionality factor. Multiplying this expression for the intensity of the energy stream by the area $a$ of a cylindrical surface $l$ centimeters in length and $r$ centimeters in radius (cylindrical surface coaxial with wire), we have the total ergs per second streaming into $l$ centimeters of the wire, and this must be equal to $l \times R I^{2}$. Therefore we have

$$
2 \pi r l \times k \times R I \times \frac{2 I}{r}=l \times R I^{2}
$$

whence

$$
k=\frac{\mathbf{I}}{4 \pi}
$$

* See Art. 19. 
Therefore, we have in general

$$
S=\frac{\mathrm{I}}{4 \pi} \mathscr{H} e
$$

in which $S$ is the energy in ergs per second which streams across one square centimeter of area at right angles to a magnetic field of which the intensity is $\mathscr{H}$ gausses and at right angles to an electric field of which the intensity is $e$ abvolts per centimeter, $\mathscr{H}$ and $e$ being at right angles to each other.

(b) The flow of energy in the neighborhood of wires assumed to be of zero resistance.-In this case the component of the electric field parallel to the surface of the wires is equal to zero, and the only electric field which exists, if any, is that which is associated with charges on the surfaces of the wires or ribbons. An ideally simple case is shown in Fig. 199, in which $A A$ and $B B$ are straight wires (ribbons), assumed to be of zero resistance, which deliver current from a voltaic cell to a fine resistance wire (ribbon) $w$. The electric field between the ribbons $A A$ and $B B$ is uniform, and the lines of force of the electric field are represented by the fine horizontal lines in the figure; the intensity of the electric field is equal to the electromotive force of the voltaic cell divided by the distance between the ribbons. The magnetic field between the ribbons is uniform, and the lines of force of the magnetic field are perpendicular to the plane of the paper in Fig. 199 as indicated by the dots between $A A$ and

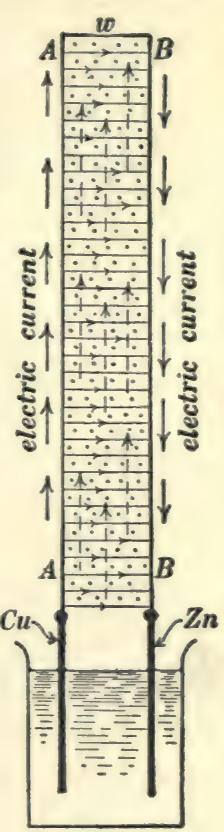

Fig. 199. $B B$. The energy stream, being everywhere at right angles to the electric and magnetic fields, is straight upwards as indicated by the dotted arrows. If the wires (ribbons) in Fig. I 99 have resistance, then the lines of force of the electric field turn slightly downwards (in the figure) near each ribbon on account of the $R I$ 
drop along the ribbons, and the energy stream, instead of flowing straight upwards, turns to some extent into the ribbons where it appears as the $R I^{2}$ loss. This is shown roughly in Fig. 200 which represents the flow of energy from a generator along a transmission line $A B$ to a distant lamp $L$. It is impracticable in this case to represent the exact trend of the lines of force of the electric and magnetic fields in the neighborhood of the generator and in the neighborhood of the lamp.*

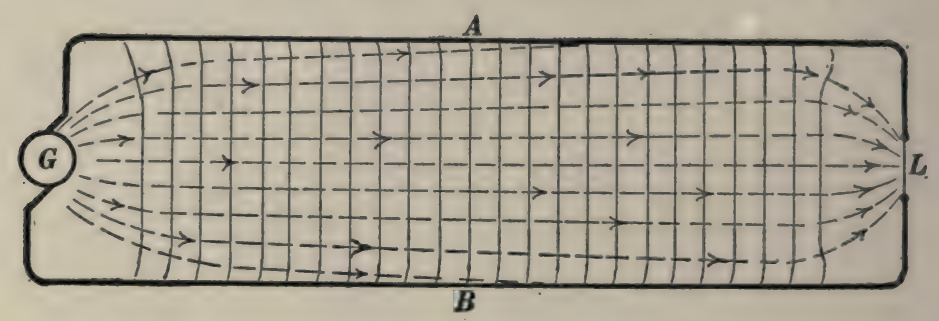

Fig. 200.

(c) The flow of energy in an electromagnetic wave.-The energy in an electromagnetic wave flows continuously from the back part of the wave to the forward part of the wave, in Fig. 194, and this energy flow is due to the coexistence of electric and magnetic fields at right angles to each other in the wave.

135. The electric oscillator.-A clear understanding of the details of action of the electric oscillator depends upon an insight into what takes place when a condenser is charged and discharged. Before discussing the electric oscillator, therefore, it is necessary to consider the charge on a condenser and its mode of disappearance when the condenser plates are connected by a wire. Consider a closed (endless) chain of gear wheels $A B$, Fig. 20I,

* Some examples of the theorem of energy flow are given in Poynting's original paper in the Philosophical Transactions for 1884 . Some interesting examples of Poynting's theorem are given by W. S. Franklin, in Physical Review, Vol. XIII, pages $165-181$, 1901. The details of field distribution and energy flow in the neighborhood of two long parallel cylindrical conductors (line wires) are given by G. Mie, Zeitschrift fur Physikalische Chemie, vol. XXXIV, page 522. 
which tend to stand in a smooth row. If the gears are allowed to slip at any point $s$, the gear $f$ being held stationary and the gear $e$ being turned in the direction of the arrow, then the chain of gears will be distorted into a zigzag row, as shown in Fig. 202. Conversely, a chain of geared cells which by elastic action tend to stand in a smooth row, will be relieved from such a zigzag

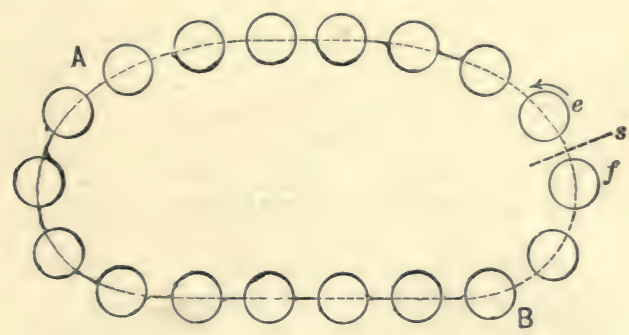

Fig. 201.

distortion as shown in Fig. 202 by permitting the gears to slip at any point $s$, and the potential energy stored in the distorted chain will be geared towards $s$ from both sides.

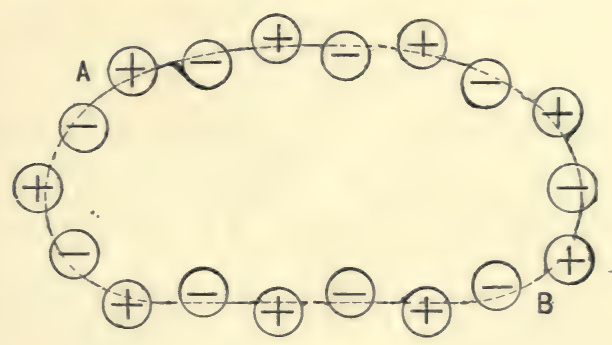

Fig. 202.

Let $A$ and $B$, Fig. 203, be two metal plates, and let the dotted lines represent closed chains of geared ether cells, each chain being like Fig. 20I. Imagine the two plates $A$ and $B$ to be connected by a wire, ${ }^{*}$ and imagine an electric current to be forced through this wire by means of a voltaic cell, thus causing

* Strictly the wire in Fig. 203 must be thought of as a broad ribbon. All figures in this discussion represent two dimensional distributions of electric and magnetic fields. 
the plates $A$ and $B$ to become charged. The forcing of electric current through the wire means a forced slipping of ether cells of each chain where it is cut by the wire, and each chain of geared cells, initially like Fig. 20I, becomes distorted like Fig. 202. Throughout the region between $A$ and $B$, the positive ether cells are displaced downwards and the negative ether

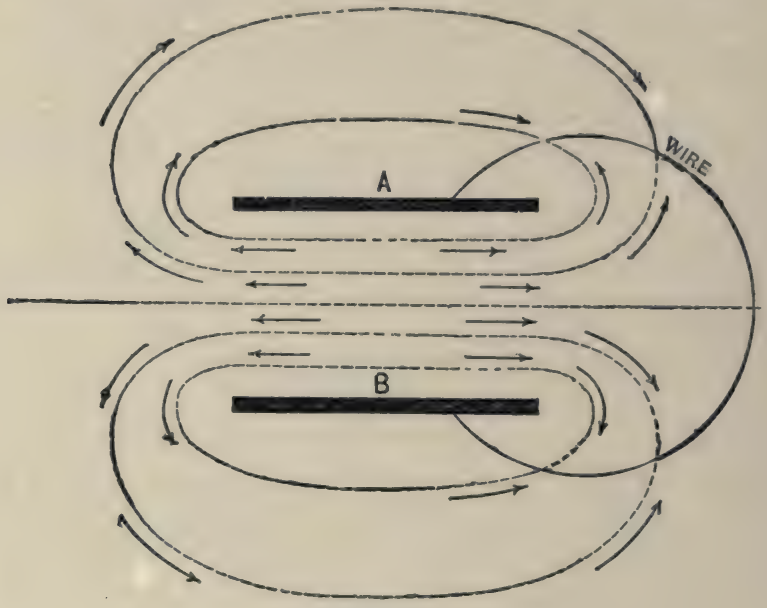

Fig. 203.

cells are displaced upwards, that is, the region between $A$ and $B$ becomes an electric field, the direction of which is downwards from the positively charged plate $A$ to the negatively charged plate $B$.

Given two charged metal plates $A$ and $B$ as above explained, each dotted curve in Fig. 203 being supposed to represent an endless zigzag chain of ether cells like Fig. 202. Then a wire (ribbon) connected from $A$ to $B$ will cut across every one of the zigzag chains of geared ether cells, slipping will begin where each chain is cut by the wire, each zigzag chain of cells will begin to be relieved from its zigzag distortion, the energy of each distorted chain will be transmitted along the chain to the wire where it will appear as heat, and the entire region between and surrounding the plates $A$ and $B$ will be relieved from 
electrical stress. The explanation here given of the entire relief of the electrical stress between two plates by the establishment of a conducting line (line of slip) between them applies to two adjacent oppositely charged bodies of any shape. An electric spark is a line of slip (a conducting line), and the energy of the electric field flows in upon the spark as it does upon a wire. The slipping of ether cells in a conductor is imagined to be opposed by a frictional drag.

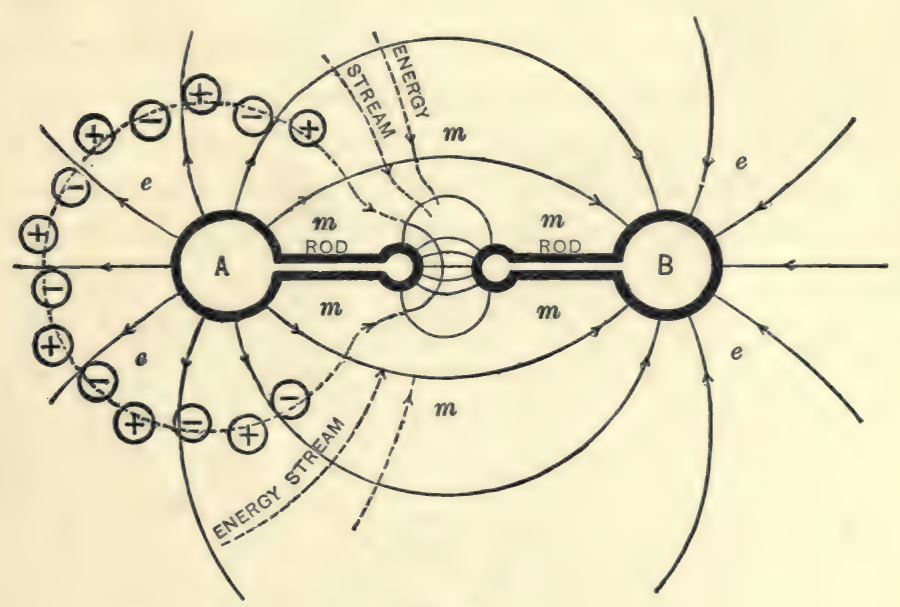

Fig. 204.

The Hertz oscillator.-Let $A$ and B, Fig. 204, be two metal balls connected to two short rods between which is an air gap (spark gap). Imagine charge to have been collecting on $A$ and $B$, positive charge on $A$, negative charge on $B$, until a spark jumps across the air gap, thus establishing a conducting path from $A$ to $B$, and causing $A$ and $B$ to be discharged. This discharge is usually oscillatory like the movement of a string which is pulled to one side and suddenly released. Consider a chain of geared ether cells which, when undistorted, lies smoothly along the dotted line in Fig. 204, this dotted line being everywhere perpendicular to the lines of force of the electric field. When $A$ is positively charged, this chain is 
distorted as shown (in part), but, inasmuch as it is a closed chain, its distortion is fixed as explained in connection with Fig. 202. When a spark is formed across the gap, however, a line of slip is established which cuts across the distorted chain, and the distortion disappears as explained in connection with Figs. 201, 202 and 203. What is said of the single chain of ether cells is true of every chain which surrounds $A$ or $B$ in Fig. 204.

If the slip which relieves the distortion of the chain of ether cells takes place with great friction (great electrical resistance of the conducting path formed by the spark), the cells near the spark begin turning slowly and the entire energy of the distorted chain is geared into the spark and converted at once into heat. If the slip which relieves the distortion of the chain of ether cells is almost frictionless (low electrical resistance of the conducting path formed by the spark), then the energy of the distorted chain is used mainly in overcoming the inertia of the cells in the neighborhood of the spark as they are set rotating, and after a very short interval of time the whole of the electrical energy will have been converted into kinetic energy of the rotating cells (magnetic energy). During this conversion the energy, streaming along the dotted lines in Fig. 204, largely disappears from the regions $e e$ and $e e$ and is distributed mainly in the region $\mathrm{mm}$. When the chains of ether cells have been relieved from distortion, the rotatory motion of the ether cells in the region $m m$ will have reached a maximum, and the cells will continue to rotate because of their momenta, thus producing reversed distortion of each chain of ether cells. During the time that this reversed distortion is being produced the energy streams back from the region $m m$ to the regions $e e$ and $e e$, being converted again into potential energy of ether distortion, and the balls $A$ and $B$ become charged in a reversed sense. This reversed distortion of the chains of ether cells is then relieved by a reversed slip (a reversed current in the rods and along the spark), and the above described action is repeated over and over again until the original energy of the electric field has been dissipated. 
When one end of a stretched rubber tube is held in the hand and moved up and down slowly, the entire tube has time to accommodate itself to the changing position of the hand. If, however, the hand is moved up and down rapidly, the portions of the tube remote from the hand do not follow the changing position of the hand promptly, and the result is that waves are produced which pass out from the moving hand. The oscillatory changes above described in connection with Fig. 204 take place so rapidly that the portions of the distorted ether which are remote from the oscillator do not follow the changes promptly. This gives rise to electrical waves which pass out from the oscillator. In the immediate neighborhood of the oscillator the action is rather complicated, but at a distance from the oscillator the wave motion becomes very simple.*

\section{PROBLEMS.}

I. A Leyden jar has walls 2 millimeters thick, the area of each tinfoil coating is 500 square centimeters, and the inductivity of the glass is 5 . What is the capacity of the jar? Ans. 0.00 I I microfarad.

2. The Leyden jar of problem I is discharged through a circuit which contains a coil of wire which consists of a winding of $\mathrm{I}, 000$ turns of wire on a cylindrical wooden rod 6 centimeters in diameter and I20 centimeters long. What is the inductance of the circuit and what is the frequency of the electrical oscillations which follow the discharge? Ans. 0.00296 henry; 882,000 cycles per second.

Note.-The influence of resistance on the frequency is to be ignored.

* Hertz's researches on electric waves, experimental and theoretical, have been published in book form (see Electric Waves by Heinrich Hertz, translated by D. E. Jones, Macmillan and Company, 1893).

A very good discussion of Hertz's experimental researches is given by J. A. Fleming on pages 306-326 of his Principles of Electric Wave Telegraphy, Longmans, Green and Company, I908.

A good discussion of the mathematical theory of the Hertz oscillator is given by Fleming on pages $326-352$ of his Principles of Electric Wave Telegraphy. This theoretical discussion of Fleming's follows the original paper by Hertz which was published in 1889 (see pages I37-I59 of Hertz's Electric Waves). 
3. The tension of a string is 25 million dynes and each centimeter of the string has a mass of 0.2 gram. What is the velocity of a wave or bend on the string? Ans. II,280 centimeters per second.

4. A transmission line consists of two copper wires each 0.5 inch in diameter and the wires are 24 inches apart center to center. (a) What is the inductance of the line per mile of the double line? (b) What is the capacity of the line per mile of double line? (c) What is the velocity of wave propagation on the line? Ans. (a) 0.00294 henry per mile; (b) $9.798 \times 10^{-9}$ farads per mile; (c) I86,000 miles per second.

5. A 10,000 volt generator of negligible resistance and inductance is switched on to the end of the line of problem 4. What is the voltage and current in the ribbon wave which shoots out along the line? Ans. Io,000 volts, I.835 amperes.

6. A battery of which the resistance is $I, 500 \mathrm{ohms}$ and of which the electromotive force is 10,000 volts is switched on to one end of the line of problem 4. What is the voltage and current in the ribbon wave which shoots out along the line? Ans. 7,852 volts and I.433 amperes.

Note.-Let $E$ be the required voltage and $I$ the required current. Then $E / I=\sqrt{L / C}$ and $E+R I=10,000$ volts, where $R$ is the resistance of the battery.

7. The line in problem 5 is 18.6 miles long and it is open at the distant end. The 10,000 volt generator is switched on to the line and disconnected after 0.00015 second. What is the current and voltage distribution over the line at the instant when the generator is disconnected? Ans. Over the half of the line next the generator the voltage is everywhere 10,000 volts and the current is everywhere 1.835 amperes; over the distant half of the line the voltage is 20,000 volts and the current is zero.

8. The distant end of the $\mathbf{I} 8.6$ mile line in problem 5 is shortcircuited and the 10,000 volt generator is disconnected (leaving the generator end of the line open) $0.000 \mathrm{I}$ second after it was 
switched on to the line. What is the current and voltage distribution over the line 0.0002 second after the generator is disconnected? Ans. Io,ooo volts opposite to generator voltage, and I.835 amperes opposite to current which first starts in the line.

9. The 18.6 mile line in problem 5 has connected across its distant end a non-inductive circuit of which the resistance is I, 826 ohms. The Io,000 volt generator is connected for 0.0015 second. What is the voltage and current distribution over the line? Ans. 10,000 volts and I.835 amperes over the half of the line next to the generator; and 5,000 volts and 2.752 amperes over the other half of the line.

10. The distant end of the 18.6 mile line in problem 5 has connected across it a non-inductive circuit of which the resistance is $16,43 \mathrm{I}$ ohms. The 10,000 volt generator is connected to the line for o.0ooro second and then disconnected. What is the voltage and current distribution over the line 0.00005 second after the generator is disconnected? Ans. Zero voltage and zero current over the half of the line next to the generator; I5,000 volts and 0.917 ampere over the distant half of the line.

II. The value of $\sqrt{L / C}$ for an air line (two wire line) is 706 "ohms," and the value of $\sqrt{L / C}$ for an underground cable is 100 "ohms." A ribbon wave in which $E=\mathrm{I}, 4 \mathrm{I} 2$ volts comes over the air line to the point where the air line connects to the cable. (a) What is the voltage and current in the wave which enters the cable, and $(b)$ what is the voltage and current in the reflected wave? Ans. $(a)+349$ volts and +3.49 amperes, (b) $-\mathrm{I}, 063$ volts and $+\mathrm{I} .5 \mathrm{I}$ amperes.

12. A battery of which the resistance is $500 \mathrm{ohms}$ and of which the electromotive force is 8,000 volts is switched on to a line for which the value of $\sqrt{L / C}$ is $\mathrm{I}, 000$ "ohms." Find the values of voltage and current in the ribbon wave which shoots out on the line. Ans. 5,333 volts, 5.333 amperes.

Note.-Let $E$ and $I$ be voltage and current in the wave. Then $E / I=1,000$ and $E+R I=8,000$, where $R$ is the resistance of the battery. 
I3. A battery of which the resistance is equal to the value of $\sqrt{L / C}$ for a given transmission line is suddenly connected to one end of the line. Describe what takes place $(a)$ when the distant end of the line is short-circuited, and $(b)$ when the distant end of the line is open.

I4. The 10,000 volt generator of problem 5 is connected to the I 8.6 mile line of which the distant end is open. Plot a curve showing the voltage across the middle of the line as a function of elapsed time, generator being left connected to the line.

I5. Plot a curve showing the current at the middle of the line in problem 14 as a function of elapsed time.

I6. Plot a curve showing voltage across the middle of the line in problem $\mathrm{I} 4$ as a function of elapsed time, the distant end of the line being short-circuited.

I7. Plot a curve showing the current in the line of problem I4 at a point Io miles from the generator as a function of elapsed time, the distant end of the line being short-circuited.

I8. Plot curves showing voltage and current at middle of line of problem 14 as functions of elapsed time, the distant end of the line being connected to a non-inductive circuit of which the resistance is $I, 826$ ohms.

I9. Plot curves showing voltage and current at a point io miles from the generator in problem 14 as functions of elapsed times, the distant end of the line being connected to a noninductive circuit of which the resistance is $16,431 \mathrm{ohms}$.

20. A single-wire line with ground return has a wire 0.25 inch in diameter hung 25 feet above the ground. What are the values of the inductance per mile and the capacity per mile of this singlewire line? Ans. 0.00274 henry per mile; 0.0105 microfarad per mile.

Note - The inductance per mile of the single-wire line with ground return is one-half the inductance per mile of a two-wire line consisting of wires 0.25 inch in diameter and 50 feet apart center to center; and the capacity per mile of the single-wire line is twice as great as the capacity per mile of the two-wire line as specified. This is on the assumption that the surface of the earth is equivalent to an infinite conducting sheet. 
21. A wave disturbance from a lightning stroke travels along the single-wire line of problem 20. Find the resistance of a lightning arrester ground connection which, if placed at the end of the line, would absorb the wave disturbance completely and reflect not at all. Ans. 5II ohms.

22. The value of the short-circuit current in Fig. 158 is, say, Ioo amperes and the value of $\sqrt{L / C}$ for the line is, say, Ioo "ohms." The inductance of the generator is assumed to be indefinitely large so that line current at the generator retains its value of roo amperes for a fairly long time after the shortcircuited line is snapped open at the distant end. The line is I 86 miles long. Plot a curve showing the voltage across the middle of the line as a function of elapsed time, neglecting effects of line resistance and leakage, and assuming the insulation strength of the line to be indefinitely great.

23. The line of problem 20 is 93 miles long and open at both ends, and it is uniformly charged (one wire positively and the other wire negatively) so that the voltage across the line is everywhere 10,000 volts and the current in the line is everywhere zero. One end of the line is suddenly closed with a switch of negligible resistance. Plot curves showing voltage and current at the middle of the line as functions of elapsed time.

24. Two oppositely moving rectangular waves $A$ and $B$ each two miles long come into superposition. The voltage in $A$ is $+5,000$ volts and the voltage in $B$ is $+2,500$ volts, and the value of $\sqrt{L / C}$ for the line is Ioo "ohms." What is the total voltage and total current in the two miles of line where the two waves come into exact superposition? Ans. $+7,500$ volts and +25 amperes.

25. Imagine a two-mile section of the line of problem 24 to have $+10,000$ volts across it everywhere and -50 amperes in it everywhere. What is the state of affairs on the line $t$ seconds later, where $t$ is the time for an electric wave to travel three miles? Ans. A section of the line two miles long in each 
direction from the center of the initial distribution will be entirely devoid of voltage and current; a two mile section beyond the 4-mile blank section and to the right will have over it a uniform voltage of $+2,500$ volts and a uniform current of +25 amperes; and a two mile section beyond the 4-mile blank section and to the left will have over it a uniform voltage of $+7,500$ volts and a uniform current of -75 amperes.

26. Calculate the leakage resistance (insulation resistance) of one mile of the line in problem 4 in order that the line might give distortionless transmission. Ans. 670,000 ohms.

Nole.-The leakage resistance here required is very much less than the leakage resistance of a well-constructed line in good condition in dry weather. In wet weather, however, the leakage resistance falls off and may give rise to approximately distortionless transmission. This is the cause of the remarkable clearness of telephonic transmission which is sometimes obtained over a moderately long line in wet weather.

27. A current of Io amperes is left to die away in a circuit of which the resistance is $2 \mathrm{ohms}$ and the inductance is $0.0 \mathrm{I}$ henry. What is the value of the current after 0.06 second? Ans. 0.0000605 ampere.

Note.-See Art. 37.

28. A ro-microfarad condenser is charged by a roo-volt battery and allowed to stand and lose its charge by leakage. The insulation resistance of the condenser is $\mathrm{I}, 000,000 \mathrm{ohms}$. What is the voltage across the condenser after 23.25 seconds. Ans. Io volts.

Note.-Let $q$ be the decreasing charge on the condenser $t$ seconds after it is left standing and let $e$ be the corresponding electromotive force across the condenser terminals. Then $q=C e$ and $\frac{d q}{d t}=C \frac{d e}{d t} ;$ but $-\frac{d q}{d t}$ is the leakage current and it is equal to $\frac{e}{R}$, where $R$ is the insulation resistance of the condenser. Therefore $\frac{e}{R}=-C \frac{d e}{d t}$ or $\frac{d e}{e}=-\frac{d t}{C R}$ so that $\log _{e} e=-\frac{t}{C R}+$ a constant.

29. A 20-mile metallic circuit telephone line consists of two No. 14 B. \& S. copper wires 18 inches apart center to center. Find the resistance of the line per mile. Find inductance and capacity of the line per mile. Find leakage resistance between 
wires of one mile of the line to give a leakage loss equal to 2 per cent of the wire loss in a pure wave. Ans. 0.00408 henry per mile, 0.00707 microfarad per mile, nnd I,032,000 ohms.

30. A I0,000-volt battery of negligible resistance is connected across the end of the line of problem 29 so as to produce on the line a rectangular wave o.I mile long. Find $(a)$ energy of wave, $(b)$ wire loss in watts, $(c)$ leakage loss in watts, $(d)$ total energy lost in wires during passage of wave to distant end of line, $(e)$ total energy lost by leakage during passage of wave to distant end of line. Ans. (a) 0.0707 joule, (b) 485 watts, $(c) 9.7$ watts, (d) $0.052 \mathrm{I}$ joule, and $(e) 0.001042$ joule.

Note.-It must not be forgotten that the total energy loss is to be calculated approximately by assuming that the rate of loss remains constant and equal to its initial value.

3r. Inductance is added to the 20-mile line of problem 30 to give a total inductance four times as great as the bare line alone. The 10,000-volt battery is connected for a sufficient time to give on this loaded line a rectangular wave o.I mile long, as in problem 30. Find $(a)$ energy of wave, $(b)$ wire loss in watts, $(c)$ leakage loss in watts, $(d)$ total energy lost in wires during passage of wave to distant end of line, and (e) total energy lost by leakage during passage of wave to distant end of line. Ans. (a) 0.0707 joule, $(b)$ I2I watts, $(c) 9.7$ watts, $(d) 0.026$ joule, and $(e) 0.00208$ joule.

32. Inductance is added to the line of problem 30 to give distortionless transmission, and the Io,000-volt battery is connected so as to give on the line a rectangular wave o.I mile long. Find $(a)$ energy of wave, $(b)$ wire loss in watts at the start, $(c)$ leakage loss in watts at the start, $(d)$ total energy lost in wires during transit of wave, assuming rate of wire loss to keep its initial value, and $(e)$ total energy lost by leakage during transit of wave, assuming rate of leakage loss to keep its initial value. Ans. (a) 0.0707 joule, $(b) 9.7$ watts, $(c) 9.7$ watts, $(d) 0.00737$ joule, and (e) 0.00737 joule. 
33. Calculate the exact values of $(d)$ and $(e)$ in problem 32 . Ans. (d) 0.00655 joule, and (e) 0.0065 joule.

Note.-The decay of current in a wave on a distortionless line takes place exactly as in an ordinary inductive circuit containing resistance, and the decay of voltage in a wave on a distortionless line takes place exactly as in a condenser that is allowed to stand. See problems 27 and 28.

34. Two No. I4 B. \& S. copper wires are laid I 8 inches apart in pure water, and the leakage resistance between wires of one mile of this line is about Io ohms. (a) Find inductance

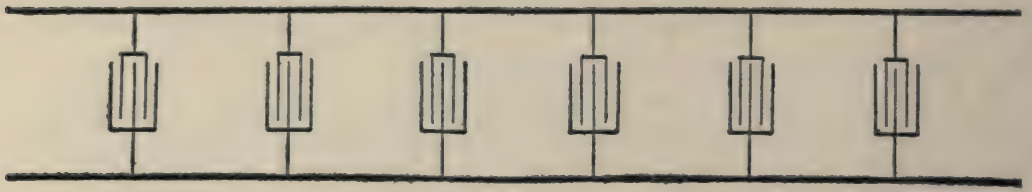

Fig. $p 33$.

and capacity of the line per mile, the inductivity of water being 90. (b) Find capacity which would have to be added per mile of line (as shown in Fig. p33) to give distortionless transmission. Ans. (a) 0.00408 henry per mile and 0.636 microfarad per mile; (b) 0.857 microfarad.

35. What fraction of the initial energy of a wave would be delivered to the end of a ten-mile line like that specified in problem 34 with capacity loading sufficient to give distortionless transmission? Ans. $2.17 \times 10^{-30}$.

Note.-See note to problem 32.

36. The string in problem 3 is 2 meters long between supports. How many vibrations per second does it make when plucked like a guitar string? Ans. 28.2 complete vibrations per second.

37. A 20-mile two-wire line is uniformly charged and set oscillating by closing a switch at one end of the line. What is the frequency of the oscillations? Ans. 2325 cycles per second.

38. What would be the frequency of the oscillations in problem 37 if both ends of the line could be closed at the same instant? Ans. 4650 cycles per second. 
39. A 20-mile transmission line consisting of two No. 8 B. \& S. wires 18 inches apart center to center and short-circuited at the distant end is connected to a I0,000-cycle alternator of which the electromotive force is 500 volts effective. Find positions of voltage nodes, and find maximum voltage between the line wires at the voltage antinodes when the ultimate steady state of oscillation of the line is established, line resistance being neglected. Also find positions of current nod es andfind maximum value of current in the line at the current antinodes. Ans. Voltage nodes at distances of $0,9.3$ and 18.6 miles from short-circuited end of line; maximum voltage at voltage antinodes I, 552 volts. Current nodes at distances of 4.65 and $\mathrm{I} 3.95$ miles from shortcircuited end of line; maximum current at current antinodes 2.293 amperes.

40. An electric field like that which is represented in Fig. I9I decreases in intensity at the space-rate of roo volts-per-centimeter per centimeter of distance along the axis of reference. (a) Find the electromotive force around a rectangle in the plane of the paper in Fig. 19I, the rectangle being 30 centimeters long (parallel to $A B$, Fig. I9I) and 20 centimeters wide. (b) Find the rate at which the magnetic flux through this rectangle must be changing in order to produce this electromotive force around it. (c) Find the rate at which the magnetic field in Fig. I9I perpendicular to the paper is changing because of the tapering of the electric field. Ans. (a) 60,000 volts; (b) $6 \times 10^{12}$ maxwells per second; $(c) \mathrm{I} \times \mathrm{IO}^{10}$ gausses per second.

4I. Consider two line wires (ribbons) $A A^{\prime}, B B^{\prime}, \quad$ Fig. $p 4 \mathrm{I}$, and imagine the current in these wires (ribbons) to be distributed so that the current at any point $p$ is represented by the ordinate $y$ of a straight line $C C$. The magnetic field between the ribbons is a tapering field as described in connection with Fig. 193 on page 250 , the lines of force of the field being perpendicular to the paper in Fig. $p 40$ and directed away from the reader. The intensity of the magnetic field in gausses at any point $p$ is equal 
to $4 \pi$ times the abamperes of current per centimeter of width of ribbons. The current at the point $p p$ is 50 amperes per unit of width, and the current at point $p^{\prime} p^{\prime}$ is zero. Find the rate at which the electric field from ribbon $A A^{\prime}$ across to ribbon $B B^{\prime}$ is increasing in volts per centimeter per second.

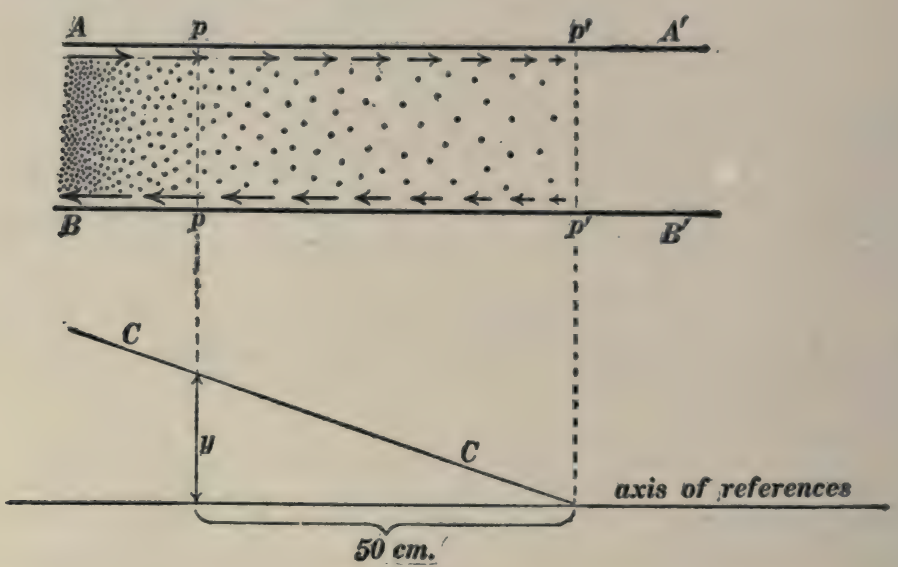

Fig. p4r.

Note.-The simplest method of handling this problem is to calculate the rate at which charge is accumulating on each square centimeter of the inner face of the ribbons due to the tapering current, positive charge on ribbon $A A^{\prime}$ and negative charge on ribbon $B B^{\prime}$, and to consider, with the help of Gauss's theorem, the intensity of the electric field which is associated with these charges as follows. Consider one square centimeter of the inner face of the upper ribbon. The current which flows into this area across one side is one ampere greater than the current which flows out of it on the other side according to the data of the problem, and therefore one coulomb of charge is collecting per second upon each square centimeter of the inner faces of the ribbons. The total electric flux which emanates from one coulomb of charge is $1.131 \times$ Io ${ }^{13}$ lines, where one line is the amount of flux crossing one square centimeter at right angles to an electric field of which the intensity is one volt per centimeter. Therefore, since one coulomb of charge is collecting per second on each square centimeter of the ribbon $A A^{\prime}$, it follows that the electric field between the ribbons is increasing at the rate of $1.131 \times 10^{13}$ volts per centimeter per second.

42. A long wire of which the resistance per centimeter of length is $0.02 \mathrm{ohm}$ carries a current of 30 amperes. (a) Find the rate at which energy flows in upon each centimeter of length of the wire in ergs per second. (b) Find the intensity of the energy 
stream in ergs per second per square centimeter at a point distant I5 centimeters from the axis of the wire. (c) Find the intensity of the electric field parallel to the wire in abvolts per centimeter. (d) Find the intensity of the magnetic field in gausses at a point distant 15 centimeters from the axis of the wire. Ans. (a) $18 \times 10^{7}$ ergs per second; (b) $\mathbf{I} .9 \mathrm{I} \times 10^{6}$ ergs per square centimeter per second; $(c) 6 \times 10^{7}$ abvolts per centimeter; $(d) 0.4$ gauss.

43. Consider two line wires in the form of two flat metal ribbons 50 centimeters wide and 3 centimeters apart. At a given point $p$ (see Fig. p43) the electromotive force between the ribbons is 100 volts and the current in each ribbon is 10 amperes. (a) Find the rate in ergs per second at which energy flows past

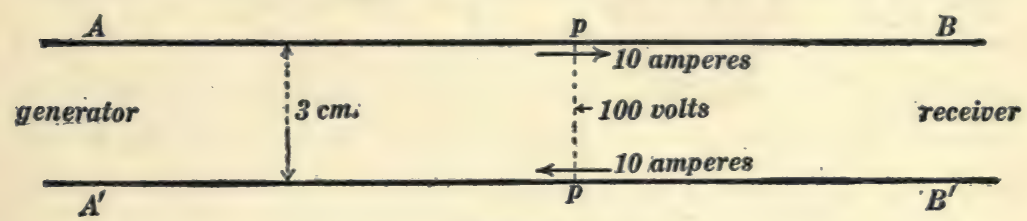

Fig. $p 43$.

the point $p$ from the generator towards the receiver using the ordinary formula, $P=E I$. (b) The electric field intensity between the ribbons at the point $p$ is 33.3 volts per centimeter and the magnetic field between the ribbons is uniform and perpendicular to the plane of the paper in Fig. p43. Find the intensity of the magnetic field in gausses. Ans. (a) $10^{10}$ ergs per second; (b) $0.25 \mathrm{I}$ gauss.

Note.-The energy which flows past the point $p$ streams through the entire region between the ribbons and therefore the sectional area of the energy stream is I50 square centimeters. Therefore the intensity of the energy stream (ergs per square centimeter per second) can be calculated; and then knowing the electric field intensity at $p$ the intensity of the magnetic field can be calculated from equation (I) of Art. I34. 


\section{CHAPTER X.}

\section{THE ELECTRON THEORY.}

\section{The mechanical theory of electricity and the atomic}

theory of electricity.-The study of electricity and magnetism as represented in the foregoing chapters is independent of any consideration of the nature of the physical action which leads to the production of electromotive force in a voltaic cell or dynamo; it is independent of any consideration of the nature of the physical action which constitutes an electric current in a wire; it is independent of any consideration of the nature of the disturbance which constitutes a magnetic field; and it is independent of any consideration of the nature of the disturbance or stress which constitutes an electric field. This kind of study of electricity and magnetism may very properly be called electro-mechanics.

Simple mechanics is the study of ordinary bodies at rest or in visible motion, and one of the most important ideas in mechanics is the idea of force; but the science of mechanics is not concerned with, and indeed it sheds no light upon the question as to the physical nature of force. Thus, the science of mechanics is not concerned with the question as to the nature of the action which takes place in a gas and causes the gas to exert a force on a piston; the science of mechanics is not concerned with the question as to the nature of the action which takes place in the material of a stretched wire causing the wire to exert a pull upon each of the two supports at its ends; the science of mechanics is not concerned with the nature of the action between the earth and a heavy weight which causes the earth to exert a force on the weight; the science of mechanics is not concerned with the nature of the action which takes place between two bodies which slide over each other and which leads to the production of the force of friction. It is sufficient for the science of mechanics 
that these actions are what may be called states of permanency of the respective systems. To speak of these actions as states of permanency is to call to mind the fact that they remain unchanged under given conditions. To say that a gas in a cylinder pushes with a force of 100 pounds on the piston thus compressing a spring is to refer to a state of affairs in which there is a clearly defined and maintained relationship between the condition of the spring and the condition of the gas. The science of mechanics owes its existence to the legitimacy and usefulness of the idea of force irrespective of the nature of the physical process upon which force action depends.

Similarly it is sufficient for the science of electro-mechanics that the physical actions that underlie electromotive force, electric current, magnetic field and electric field are what may be called states of permanency. Thus to say that a dynamo produces a current of 100 amperes through a circuit is to refer to a state of affairs in which there is a definite and maintained relationship between the dynamo and the circuit, the dynamo delivers energy at a certain rate and the circuit receives energy at a certain rate, and the circuit exhibits to a definite degree the various effects which are associated with what we call an electric current. The science of electro-mechanics owes its existence to the legitimacy and usefulness of the ideas of electromotive force, electric current, magnetic field and electric field irrespective of the nature of the physical actions upon which these various things depend.

The superficial character of the science of simple mechanics and of the science of electromechanics may be further exemplified as follows: A sample of steel is broken by a tension of 120,000 pounds, but the exact character of the action which takes place in the steel when it is placed under tension is not a matter for consideration. Neither does one need to consider the action which takes place in the furnace of the boiler which supplies steam to the engine which drives the dynamo which supplies current to the motor which drives the testing machine! A plate 
of glass is broken down and punctured by an electromotive of 95,000 volts, but the exact character of the action which takes place in the glass when it is subjected to the electromotive force is not a matter for consideration, nor is it necessary to consider the changes which take place in the battery, for example, which may be used to produce the given voltage. Simple mechanics is concerned with the correlation of what may be called lump effects, such as the relationship between the size of a beam and the load it can carry, the size of a fly wheel and the work it can do when stopped, the thickness and diameter of a boiler shell and the pressure it can stand, the size of a submerged body and the buoyant force which acts upon it, the size and shape of the air column in an organ pipe and its number of vibrations per second, the thickness of a glass plate and the electromotive force it can stand, the size of a copper wire and the current it can carry with a given rise of temperature and so forth.

THE ATOMIC THEORY.-The atomic theory depends upon the development of more or less hypothetical conceptions of the minute details of physical action. Thus the burning of hydrogen is thought of as the joining together of atoms of hydrogen and oxygen; a gas is thought of as made up of an enormous number of particles which fly around at random and exert pressure on an exposed surface by bombardment.

The phenomena of chemical action and those physical phenomena which have to do with the minute details of physical processes have been studied heretofore almost solely on the basis of the atomic theory. Thus nearly the whole of chemistry is based upon the atomic theory, the kinetic theory of gases is a branch of atomic theory; the theory of crystal structure is a branch of atomic theory, and the atomic theory is used to a very considerable extent in studying electricity and magnetism and especially in the study of the discharge of electricity through gases and in the study of radio-activity.

137. Electrons and ions. - The loss of electricity by a charged body has long been known to be due in part to a leakage of 
the electricity through the surrounding air and in part to a leakage of the electricity through the insulating supports of the charged body, that is to say the air conducts electricity to some extent. Ordinarily, however, the air is an extremely poor conductor, but there are a number of influences which cause the air (or any gas) to become a fairly good electrical conductor. Thus gas which is drawn from the neighborhood of a flame or from the neighborhood of glowing metal or carbon is a fairly good conductor; gas which has been drawn from a region through which an electric discharge has recently passed is a fairly good conductor; a gas becomes a fairly good conductor under the action of Roentgen rays or under the action of the radiation from radioactive substances. The conductivity which is imparted to a gas by these various agencies may be destroyed by filtering the gas through glass wool or by placing the gas for a few moments between electrically charged metal plates. This effect of filtration seems to show that the conductivity of the gas is due to something which is mixed with the gas, and the effect of the electric field (between the two charged plates) seems to show that this something is charged with electricity and is dragged out of the gas by the electric field. From some such considerations as these the hypothesis was originated that the electrical conductivity of a gas is due to electrically charged particles floating around in the gas. These particles are called ions, and the process by which a gas is made into a conductor is called ionization. This hypothesis has been used extensively and with remarkable success in the study of electrical discharge through gases and in the study of radio-activity, and it is no longer thought of as a mere hypothesis.

The electron is a negatively charged particle of which the mass is about $I / 800$ of the mass of a hydrogen atom. The cathode rays, which are described later, are electrons thrown off from the cathode of the Crooks' tube at high velocity, the $\beta$-rays from a radio-active substance such as uranium are electrons which are expelled at extremely high velocity from the atoms of the substance. 
An ion is an atom of a gas from which a negatively charged electron has been detached leaving the remainder of the atom positively charged, or an atom to which an extra electron has become attached thus giving the atom a negative charge. For example the canal rays in a Crooks' tube consist of positively charged atoms of the residual gas and the $\alpha$-rays which are given off by a radio-active substance such as uranium are positively charged atoms.

I38. Ionization by the electric field.-According to the kinetic theory of gases, a molecule of a gas travels on the average a certain distance between successive collisions with neighboring molecules. This distance is called the mean free path of the molecule. The mean free path of an electron in a gas is about $4 \sqrt{2}$ times as great as the mean free path of the molecule of the gas, ${ }^{*}$ because of the very small size and great velocity of the electron, whereas the mean free path of an ion is equal to or even less than the mean free path of the molecule of the gas. When a gas is subjected to an electric field by being placed between two oppositely charged metal plates, a certain amount of energy is imparted by the electric field to the electrons between successive collisions, and a much smaller amount of energy is imparted to the ion between successive collisions (because of their shorter mean free path). If the energy imparted to an electron between successive collisions exceeds a certain value, the electron is able to ionize an atom of the gas at its next collision, and when an atom of the gas is thus ionized a new electron and a new ion are produced. Similarly if the energy imparted to an ion between successive collisions exceeds a certain value, the ion is able to ionize an atom of the gas at its next collision thus producing a new ion and a new electron. Experiment shows that an electron must fall freely through a difference of potential of about 30 volts

* The discussion of the electron theory in this chapter is necessarily very brief. The student who is interested in the subject should read Sir J. J. Thomson's Conductivity of Electricity through Gases, and Sir Ernest Rutherford's Radio-active Transformations. 
in order to receive enough energy to enable it to ionize an atom of oxygen or nitrogen, and an ion must fall freely through a difference of potential of about 440 volts in order to receive enough energy to enable it to ionize an atom of oxygen or nitrogen.

I39. The electric spark in a gas.-When a gas is subjected to an electric field of which the intensity is sufficient to cause both* the electrons and the positive ions to ionize the gas, an extremely rapid and unlimited increase in the number of electrons and ions takes place, and the result is the production of an electric spark. The mean free path of the positive ions in a gas is inversely proportional to the pressure of the gas so that the electric strength of a gas should be approximately proportional to the pressure. This is, in fact, the case. Thus, the dielectric strength of air at normal atmospheric pressure is about 32,000 volts per centimeter, at a pressure of ro atmospheres the strength is about 320,000 volts per centimeter, and at a pressure of 0.1 atmosphere the dielectric strength is about 3,200 volts per centimeter. The dielectric strength of air reaches a minimum, however, at a pressure of about 2 millimeters of mercury, and increases when the pressure falls below this value. An electromotive force, sufficient to produce a spark $\mathrm{I} / 8$ of an inch long in air at atmospheric pressure, will produce a discharge through 18 or 20 inches of air at 2 millimeters pressure.

The idea of dielectric strength is based on the assumption that the electromotive force required to produce a discharge is proportional to the length of the spark, so that the quotient, volts divided by spark length, may be a constant. This is only

* When the intensity of an electric field is sufficient to cause only the electrons to ionize the gas, then all of the electrons form new ions and new electrons as they travel towards the positive electrode, and when they reach the positive electrode the action ceases except for the occasional formation of a stray electron by outside influences. When the electric field is sufficiently intense to cause electrons and positive ions both to produce ionization, then new ions and electrons are formed everywhere between the electrodes, and the number of free ions and electrons increases indefinitely. It is a well-known fact that an electric field must continue to act for an appreciable time before a spark is produced. 
approximately true in gases under moderate or high pressure, and when the pressure is very low a greater electromotive force is required to strike across a short gap than is required to strike across a long gap. This curious behavior of gas at low pressure

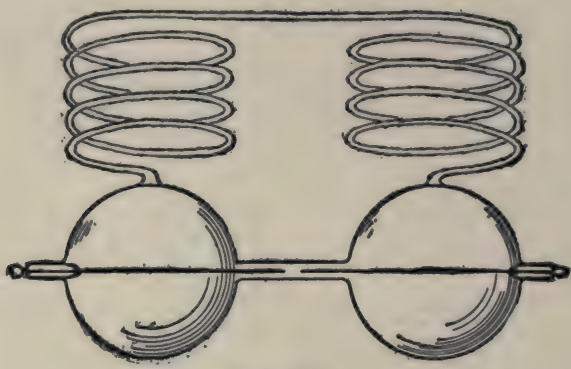

Fig. 205.

is illustrated by a famous experiment due to Hittorf. Two electrodes were sealed into the walls of two glass bulbs and the tips of the electrodes were one millimeter apart, as shown in Fig. 205. The two bulbs were connected together by a spiral tube 375 centimeters long, and, when the pressure of the gas in the bulbs was reduced to a very low value, the discharge took place through the long tube and not across the one millimeter gap space between the points of the electrodes.*

I40. The Geissler tube and the Crookes tube.-The discharge of electricity through gases at low pressures is usually studied by means of a glass bulb through the walls of which are sealed platinum wires which terminate in metal plates called electrodes. The current enters at one electrode, the anode, and passes out at the other electrode, the cathode. This bulb, which is called a vacuum tube, is filled with the gas to be studied and the pressure is reduced to any desired value by exhausting the tube by means of an air pump.

Before exhaustion the discharge through the tube is in the form of a sharply-defined spark similar to the spark in the open

* This behavior of a gas at low pressure is fully explained by the atomic theory. See J. J. Thomson's Conduction of Electricity through Gases, pages 430-527. 
ai:- When the pressure of the gas in the bulb has been reduced to a few centimeters of mercury, the spark begins to be nebulous, and a continued reduction of pressure causes the luminosity ultimately to fill the entire tube. When the pressure has been reduced to a few millimeters of mercury the discharge presents the following features, as shown in Fig. 206. There is a thin layer of luminosity spread over the surface of the cathode $C$, and beyond this there is a comparatively dark space $D$ called the Crookes dark space, the width of which depends upon the pressure of the gas, increasing as the pressure of the gas diminishes. This Crookes dark space extends to a boundary which is approximately a surface traced out by lines of constant length drawn normally to the surface of the cathode. Beyond the Crookes dark space is a luminous region $N$ called the negative glow, and beyond the negative glow is another comparatively dark region $F$ which is called the Faraday dark space. Beyond the Faraday dark space is a luminous column $P$ extending to the anode $A$ and called the positive column. This positive column usually exhibits alternations of bright and dark spaces which are called striations. The effects here descibed are exhibited at their best in a vacuum tube in which the pressure has been reduced to a few millimeters of mercury. Such a vacuum tube is called a Geissler tube. When the exhaustion of the vacuum tube is carried further, the dark space which surrounds the cathode (the

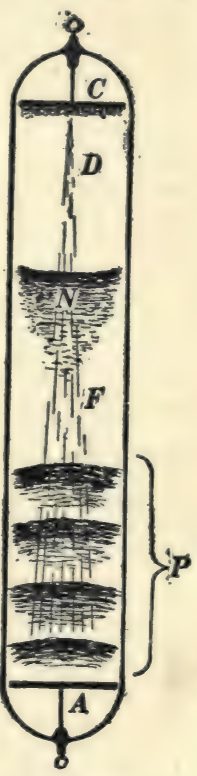

Fig. 206. Crookes dark space) expands until it fills the entire tube. The glass walls of the tube then show a yellowish-green or blue luminescence (according as the tube is made of soda glass or lead glass) and a slight negative glow may remain in portions of the tube remote from the cathode. These effects, which were first studied by Crookes in England and by Plücker and Hittorf in Germany, are exhibited at their best in a vacuum 
tube in which the pressure has been reduced to a few thousandths of a millimeter of mercury. Such a vacuum tube is called a Crookes tube.

I4I. Cathode rays and canal rays. - In order that a steady discharge may flow through a vacuum tube, it is necessary that the electric field intensity reach a value sufficient to impart to the positive ions enough energy between collisions to enable them to ionize the molecules of the residual gas, because if the electrons (negative ions), only, produce ionization, the discharge through the tube ceases very soon after all of the negative ions have moved across to the neighborhood of the anode. In fact, ionization by positive ions must take place in the neighborhood of the cathode, ${ }^{*}$ and it is this necessity which gives rise to the Crookes dark space. The action which takes place in the Crookes dark space is as follows: Electrons (negative ions) are thrown off from the cathode at very high velocity by the intense electric field in the Crookes dark space, very energetic ionization takes place in the negative glow $N$, Fig. 206, and the positive ions that are produced in this region attain sufficient velocity in traveling towards the cathode to enable them to ionize the gas in the immediate neighborhood of the cathode. That is, ionization by positive ions takes place in the faint glow which covers the cathode. The mutual dependence of the ionization which takes place in the negative glow and the ionization which takes place in the faint luminosity in the immediate neighborhood of the cathode is shown by placing a small obstacle in the Crookes dark space. This obstacle screens a portion of the cathode surface from bombardment by the positive ions which move from the negative glow towards the cathode so that the region so shaded is free from even faint luminosity because ionization does not take place there. In the same way the obstacle also screens a certain portion of the negative glow from bombardment by the electrons which are thrown from the cathode and this portion of

* A detailed discussion of this matter may be found in J. J. Thomson's Conduction of Electricity through Gases, pages 529-603. 
the negative glow ceases to exist because ionization is no longer produced there. That is to say, the obstacle casts a shadow on the cathode and it also casts a shadow into the negative glow.

The electric field intensity in the Crookes dark space, being necessarily sufficient to enable the positive ions to produce ionization at the surface of the cathode, is able to impart very much greater velocity to the electrons than is necessary to enable them to produce ionization. The result is that the electrons which are thrown off from the cathode travel in straight lines through a long portion of the tube. These high velocity electrons constitute what are called cathode rays. The cathode rays are faintly visible throughout the tube because of occasional collisions with the molecules of the gas.

When the cathode has a small hole through it, the positive ions which move towards the cathode from the negative glow pass

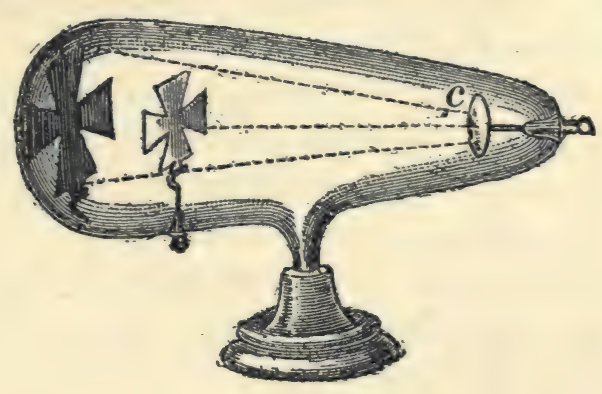

Fig. 207.

through this hole in the form of a stream of rays which is made visible by the luminosity which accompanies the collisions of the positive ions with the molecules of the gas. Such a stream of positive ions constitutes what has been called the canal rays.

An object of any kind placed in the Crookes tube casts a sharp shadow upon the wall of the tube, as shown in Fig. 207. The wall of the tube shows a brilliant luminescence everywhere except where it is screened by the obstacle from bombardment by the cathode rays. 


\section{I42. Magnetic deflection of cathode rays and canal rays.-} A moving charged body is equivalent to an electric current, and when a charged body moves across a magnetic field the magnetic field pushes sidewise upon the charged body and causes the charged body to describe a curved path. The magnetic deflection of the cathode rays is easily shown by placing a horse-shoe magnet with its poles placed on opposite sides of the tube shown in Fig. 207. The shadow of the cross is thrown up or down according to the arrangement of the magnet. The magnetic deflection of the canal rays is very slight; a very strong magnetic field is necessary to produce a perceptible deflection. The direction of the magnetic deflection of the cathode rays shows that these rays are negatively charged particles, and the direction of the magnetic deflection of the canal rays shows that these rays are positively charged particles. The magnitude of the deflection of the cathode rays shows that the mass of the cathode particles (electrons) is very small and that their velocity is very great. The magnitude of the deflection of the canal rays shows that the mass of the canal ray particles is relatively great and that their velocity is less than the velocity of the cathode rays. This matter is explained in detail in Art. 148.

An object upon which the cathode rays* impinge is heated, it may be, to a very high temperature. Many substances, however, emit light (without being made perceptibly hot) when subjected to bombardment by the cathode rays. Such substances are said to be luminescent. For example, lead sulphate emits a deep violet light, zinc sulphate emits white light, magnesium sulphate, with a slight admixture of manganese sulphate, emits a deep red light under the action of cathode rays.

The cathode rays pass quite readily through thin metal plates, especially through thin plates of aluminum. By using a Crookes

* The cathode rays produce effects which are practically important and which can be easily observed. The effects of the canal rays, however, are so slight as to be scarcely perceptible even under the most favorable conditions. Therefore further discussion of the canal rays is not warranted in this brief outline. 
tube of which a portion of the wall is made of thin sheet aluminum, the cathode rays may be made to pass through into the outside air. The properties of cathode rays thus obtained in the air were first studied by Lenard who found that they produce a very high degree of ionization of the air making it a fairly good electrical conductor. Lenard found the cathode rays capable of traversing from to to 20 centimeters of air at atmospheric pressure, he found them capable of exciting luminescence, and he found them capable of affecting a sensitive photographic plate.

I43. The Roentgen rays.-Objects upon which the cathode rays impinge, not only become heated and luminescent as described above, but they emit a type of radiant energy which was discovered by Roentgen in 1894 . Roentgen rays are of the same physical nature as light rays, that is, they consist of waves in the ether, and they are related to light waves very much as a sharp "razor" wave on the surface of water would be related to a long ocean swell as shown in Fig. 208.* Helmholtz pointed out

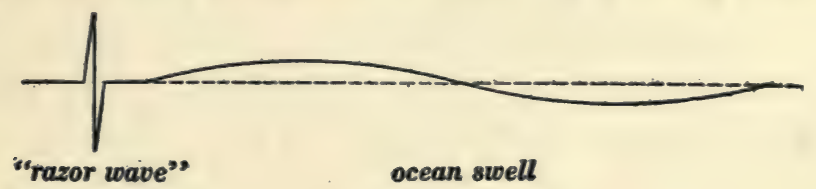

Fig. 208.

in I89I that abrupt wave pulses of this kind in the ether would have certain properties, the properties, in fact, which are exhibited by Roentgen rays, as follows: These rays are not reflected in a regular way by the surface of a mirror, and they are not refracted by a lens. They pass through all substances, subjected to a certain amount of absorption which is greater the greater the density of the substance, and subject to a certain amount of diffused scattering. The Roentgen rays affect an ordinary photographic plate and they have a powerful ionizing effect on gases.

*Very recent investigations seem to show that the Roentgen rays are not isolated abrupt rays but trains of waves of very short wave length. 
The fluoroscope.-Many substances such as barium platinocyanide and calcium tungstate become luminescent under the action of Roentgen rays. This effect is utilized in the fluoroscope which consists of a cardboard screen covered with a layer of barium platinocyanide. When the Roentgen ray shadow of an object, such as the hand, falls on this screen the shadow becomes visible; where the Roentgen rays have been greatly reduced in intensity by the bones of the hand the screen remains dark, where the Roentgen rays have been slightly reduced in intensity by the flesh the screen is moderately luminous, and where the rays have not been reduced at all in intensity the screen is highly luminous. The Roentgen ray shadow of an object may be rendered visible by allowing it to fall upon a photographic plate and developing the plate as in ordinary photography.

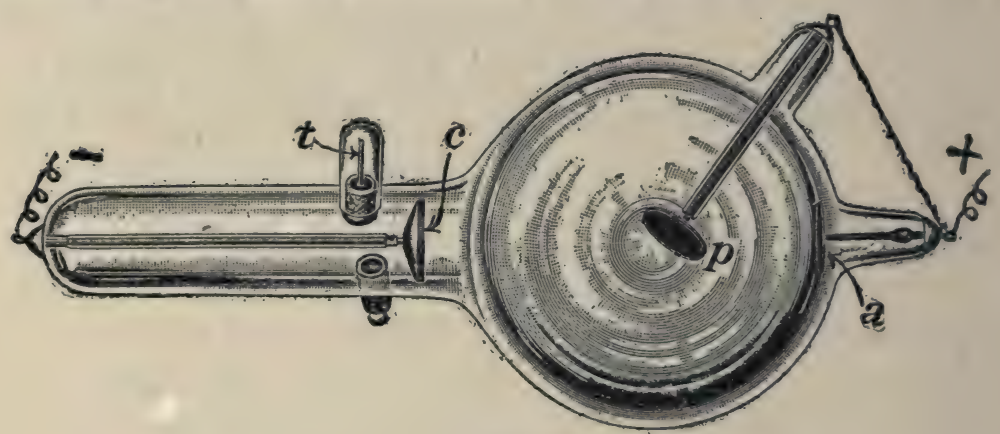

Fig."209.

The focusing tube. - In order that a shadow may be sharply defined the radiation which produces the shadow must emanate from a very small source. Figure 209 shows a Crookes tube with a concave cathode $c$ from which the cathode rays are thrown off in a convergent stream which strikes a small spot on a platinum plate $p$. This small spot is the source of the Roentgen rays. Such a Crookes tube is called a focusing tube, and, by the use of such a tube, very sharply defined Roentgen ray shadows may be produced. The platinum plate $p$ is usually connected as shown to the aluminum anode $a$. An interesting feature of the Crookes 
tube, which is shown in Fig. 209, is the small platinum tube $t$ which is sealed through the glass wall. When the vacuum in the Crookes tube becomes too high (presumably by the transformation of the residual gases into non-volatile products), the small tube $t$ is held for a few seconds in the flame of an alcohol lamp and a sufficient amount of hydrogen passes through the hot platinum to replenish the supply of gas in the Crookes tube.

144. Conductivity of flames.-A flame is a fairly good electrical conductor and this conductivity has been found to be due to the presence of free ions.* The conductivity of a flame is shown by the fact that a charged glass rod may be completely discharged by passing the flame of a Bunsen burner rapidly over its surface.

I45. The electric arc.-In order to produce a perceptible discharge of electricity (flow of current) through a gas, a very high electromotive force must be used because of the necessity of producing ionization in the gas by the collision of the moving ions with the gas molecules; and the amount of current which can be made to flow through a gas is usually very small because of the comparatively small number of these ions. When, however, metal or carbon electrodes are heated to a very high temperature they emit electrons (negative ions) in great numbers $\dagger$ and a very considerable current may then be made to flow through the intervening gas. Thus, a current of an ampere or more may be made to flow between a cold metal anode and a very hot metal cathode in a vacuum tube. When two carbon rods are connected to a battery or dynamo, brought into contact and then separated, the current which begins to flow across the indefinitely small gap between the two carbon rods raises the tips of the carbons to a very high temperature so that electrons (negative ions) are emitted in great numbers. The result is that the current continues to flow between the carbon tips. The column of hot

* See J. J. Thomson's Conduction of Electricity Through Gases, pp. 228-249.

† See J. J. Thomson's Ccnduction of Electricity Through Gases, pp. 188-227. 
ionized vapor between the carbon tips is called an electric arc, and the intense heating of the two carbon tips is due to their bombardment by the ions which move across the arc and carry the electric current. The electric arc may be easily maintained between a hot carbon cathode and a rapidly rotating disk (a cold

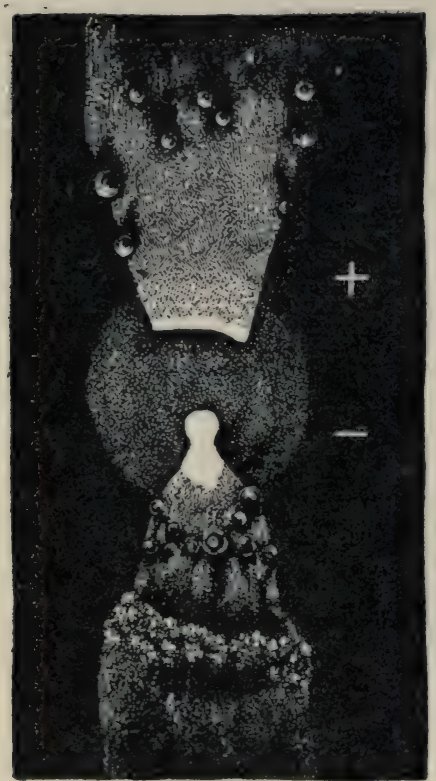

Fig. 210. anode), but not between a cold cathode and a hot anode. This shows that the emission of negative ions (electrons) by the hot cathode is essential to the formation of the electric arc. The appearance of the arc between carbon electrodes is shown in Fig. 210.*

146. Chemical effect of the discharge through gases.-The discharge of electricity through gases is accomplished by the ionization of the gas as above explained. This ionization means not only the breaking down of the molecules of a compound gas but also the separation of electrons from individual atoms. The ionization of mixed gases promotes chemical combination. Thus the nitrogen and oxygen of the air combine slowly under the action of the electric spark.

When oxygen (or air) is ionized, the recombination of the oxygen ions results in the production of ozone. Thus the peculiar odor which is given off by a Toepler-Holtz machine or a Wimshurst machine is due to the ozone which is formed. The action which takes place in the formation of ozone from oxygen

* The properties of the electric arc are discussed in great detail in a paper by C. P. Steinmetz, Transactions International Electrical Congress, Vol. II, pages 7 ro730, St. Louis, 1904; in a paper by W. R. Whitney, Transactions American Electrochemical Society, Vol. 7, pages 291-299, 1905; and in J. J. Thomson's Conduction of Electricity Through Gases, pages 604-620. 
\&s as follows: Ordinary oxygen is bi-atomic, that is, it contains two atoms of oxygen in the molecule. Ionization causes the disintegration of these bi-atomic molecules forming ions of monoatomic oxygen, and this mono-atomic oxygen recombines forming a large proportion of bi-atomic oxygen again and a small proportion of tri-atomic oxygen, or ozone. In the production of ozone for commercial purposes a blast of air is driven between two metal plates which are connected to a high voltage alternator. The repeated reversals of the high electromotive force between the plates ionizes the intervening air repeatedly, and the recombination of the ions is accompanied by the formation of a certain percentage of ozone, as above explained. In order to produce a high degree of ionization throughout the entire region between the two metal plates, it is necessary to place a thin plate of glass between the metal plates so as to prevent the formation of a single spark from plate to plate. The effect of this glass plate is to cause a corona discharge to take place throughout the entire region. Without the glass plate a single brilliant spark passes through the air; with the glass plate, a diffused violet luminosity is produced throughout the region between the metal plates.

I47. Radio-activity. *-The chemical elements uranium, thorium, and radium and their compounds have the property of making a surrounding gas an electrical conductor. Thus, one ten-millionth of a gram of radium bromide which is left as a residue upon a metal plate by evaporating a small quantity of a dilute solution of radium bromide on the plate, causes a gold leaf electroscope to be discharged in a few seconds when the radiumcovered plate is held near to the metal plate of the electroscope. Uranium and thorium have the same effect but the discharge

* The student is referred to the following books for a full discussion of radioactivity: Radioactivity, by E. Rutherford, Cambridge, 1905 (second edition); Radioactivity, by Frederick Soddy, London, I904; and Radioactive Transformations by E. Rutherford, New York, I906. See also Rutherford's Radioactive Substances and their Radiations, Cambridge, I9r3. 
which they produce is not so rapid unless a large quantity of material is employed. This property of these metals and of their compounds is called radio-activity, a name which originated because of the peculiar radiations which are given off by these substances and to which the discharging action is due. These radiations are of three distinct kinds, which are called the $\alpha$-rays, the $\beta$-rays, and the $\gamma$-rays, respectively. The $\gamma$-rays penetrate through a foot or more of solid metal or through many feet of air; the $\beta$-rays penetrate through a moderate thickness of a light metal, such as aluminum; whereas the $\alpha$-rays are stopped by a very thin layer of aluminum or by a layer of air two or three inches in thickness.

The $\alpha$-rays consist of positive ions each about four times as massive as a hydrogen atom. These ions are projected from the radioactive substance at a velocity of about 20,000 miles per second, and each of them ionizes about Ioo,000 air molecules before it is brought to rest by repeated collision. After traveling two or three inches through the air, the velocity of these $\alpha$-particles is reduced to so low a value as to render them no longer perceptible by their ionizing effects.

The $\beta$-rays consist of electrons (negative ions) each about I/800 as massive as a hydrogen atom. These electrons are projected from the radio-active substance at a velocity which in some cases is nearly as great as the velocity of light (I86,000 miles per second). The $\beta$-particles also have the property of ionizing the gas through which they pass but not to so great an extent as the $\alpha$-particles, and they travel several feet through the air before their velocity is reduced to so low a value as to render them no longer perceptible by their ionizing effects.

The $\gamma$-rays are extremely abrupt waves in the ether essentially the same in character as Roentgen rays, but much more penetrating than ordinary Roentgen rays. The $\gamma$-rays also have the property of ionizing a gas.

The $\alpha$-rays and the $\beta$-rays are deflected by the magnetic field and by the electric field. The direction of the deflection of the 
$\alpha$-rays is in each case opposite to the direction of deflection of the $\beta$-rays, and therefore it is known that the $\alpha$-particles are positively charged and that the $\beta$-particles are negatively charged. The $\gamma$-rays are not deflected by a magnetic field or by an electric field.

The present hypothesis regarding radio-activity is that the atom of any substance is a system of excessively small negatively charged particles, called electrons, revolving around a small positive charge or in a small region containing positive charge, the atom of each element being a characteristic group or system. These systems of electrons (atoms) are supposed to be to some extent unstable, and when instability occurs, the system (atom) collapses into a new configuration, and at the same time expels one or more positively or negatively charged electrons or groups of electrons which constitute the

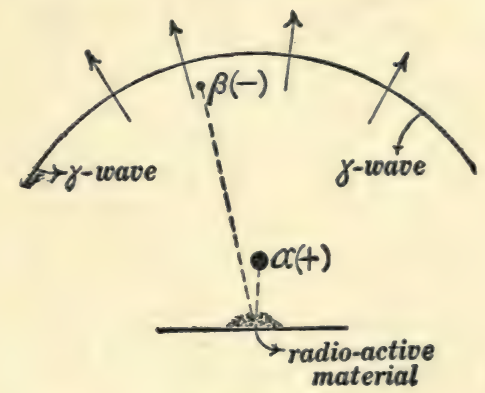

Fig. 211.

Showing the relative distances traveled by the $\gamma$-wave, by the $\beta$-particle and by the $\alpha$-particle in a given time.

$\alpha$-rays and the $\beta$-rays. According to this hypothesis the $\gamma$-rays consist of abrupt ether waves which are produced by the sudden collapse of the atomic structure when instability occurs. ${ }^{*}$ A clear representation of the nature of $\alpha-, \beta$-, and $\gamma$-rays is shown in Fig. 2II. Imagine an atom of the radio-active material to

* A very instructive discussion of the electron theory is given by Sir Oliver Lodge in a book entitled Electrons, published by Geo. Bell \& Sons, London, 1906. The method of measuring the degree of radio-activity of a radio-active substance is explained in the books of Soddy and Rutherford above referred to. 
collapse at a given instant sending out a $\gamma$-wave, an $\alpha$-particle, and a $\beta$-particle. The relative positions reached by the $\gamma$-wave, the $\alpha$-particle, and the $\beta$-particle at a given instant are shown in the figure. The $\alpha$-particle is a large positively charged particle and the $\beta$-particle is a small negatively charged particle.

148. Determination of velocity and mass of the particles which constitute canal rays (or $\alpha$-rays) and cathode rays (or $\beta$-rays).-A narrow stream of rays from a radio-active substance may be obtained by the arrangement shown in Fig. 212 in which

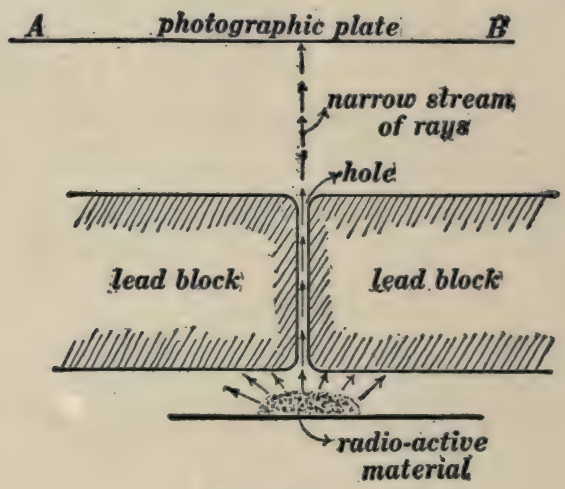

Fig. 212.

$A B$ is a sensitive photographic plate upon which the narrow stream of rays impinges. Figure 213 shows the effect of an electrical field upon a thin stream of rays from a radio-active substance. The direction of the electric field is shown by the fine horizontal arrows (the lines of force of the electric field pass from the positively charged plate to the negatively charged plate). The effect of the electrical field is to deflect the $\alpha$-particles in the direction of the field and the $\beta$-particles in the opposite direction, while the $\gamma$-rays are not deflected at all. The amount of deflection in each case may be determined by developing the photographic plate upon which the rays impinge. The effect of the magnetic field upon the rays from a radio-active substançe is shown in Fig. 214 in which the fine horizontal lines 
represent the lines of force of a magnetic field between the two large magnet poles.
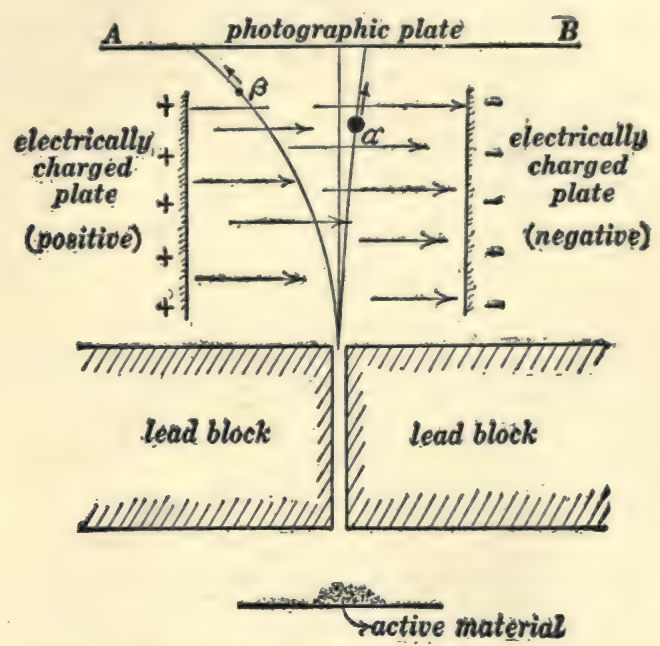

Fig. 213.

The determination of the velocity of the $\alpha$ - and $\beta$-particles is somewhat analogous to the following method of determining the velocity of a cannon ball. The curved line in Fig. 215 repre-

A. photographic plate B

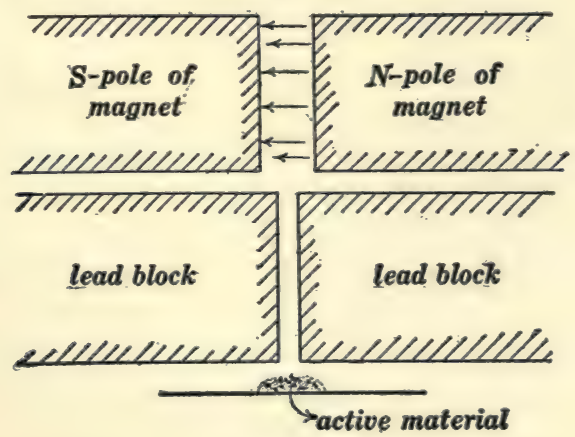

Fig. 214.

$\alpha$-particles deflected towards the reader and $\beta$-particles deflected away from the reader. 
sents the orbit of a cannon ball, $D$ being the horizontal distance traveled by the ball in a given time and $d$ being the vertical distance fallen by the ball under the action of gravity. If $D$

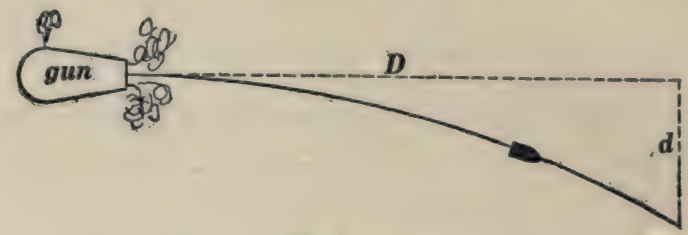

Fig. 215.

is known and $d$ observed, then the velocity of the cannon ball is given by the equation:

$$
v^{2}=\frac{g D^{2}}{2 d}
$$

in which $g$ is the acceleration of gravity.

Action of the electrical field on a moving charged particle.Consider a charged particle moving upwards through an electrical field as shown in Fig. 2I6. Let $g$ be the charge on the

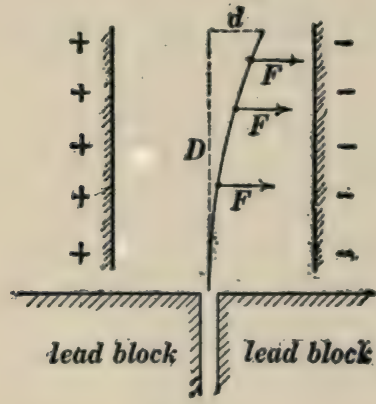

Fig. 216.

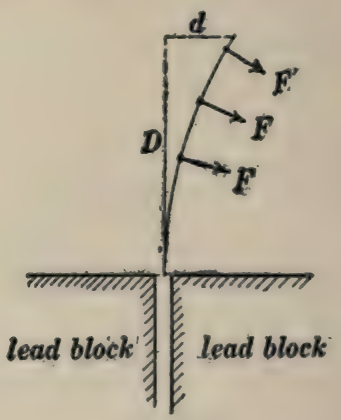

Fig. 217.

Electric field from right to left.

particle in abcoulombs and $e$ the intensity of the electrical field in abvolts per centimeter. Then the force $F$ in dynes pulling sidewise on the particle is equal to $q e$, so that the acceleration of the particle in the direction of $F$ is $q e / m$, where $m$ is the mass of the particle in grams. It is evident that the particle moves 
in the same sort of an orbit as a cannon ball, and that the acceleration $q e / m$ corresponds to the acceleration of gravity $g$ in the case of a cannon ball. Therefore, using $q e / m$ for $g$ in equation (I), we have

$$
v^{2}=\frac{D^{2} q e}{2 d m}
$$

or

$$
\frac{q}{m}=\frac{2 d v^{2}}{D^{2} e}
$$

Action of the magnetic field on a moving charged particle.Figure 217 represents a charged particle moving upwards through a magnetic field, the lines of force of which are perpendicular to the plane of the figure. The moving particle is equivalent to an electric current, and the side force $F$ is equal to $q v h$ where $q$ is the charge on the particle in abcoulombs, $v$ is its velocity in centimeters per second, and $h$ is the intensity of the magnetic field in gausses. Therefore the acceleration of the particle in the direction of $F$ is $q v h / m$. The force $F$ is continuously at right angles to $v$ so that the particle describes a circular orbit. But the acceleration of a particle moving in a circular orbit is $v^{2} / r$, and the relation between the radius of the circle $r$, the semichord $D$, and the versed sine $d$ is

Therefore we have

$$
r=\frac{D^{2}}{2 d}
$$

$$
\frac{q v h}{m}=\frac{2 d v^{2}}{D^{2}}
$$

whence

$$
\frac{q}{m}=\frac{2 d v}{h D^{2}}
$$

Determination of velocity of particles.-Reduced to the simplest terms, the method of determining the velocity may be described as follows: An electric field $e$ in the plane of the paper, Fig. 216, and a magnetic field $h$ at right angles to the plane of the paper in Fig. 217 are adjusted so that together they produce 
no deflection of the particles which are being studied. When this condition is realized, the force $q e$ with which the electrical field acts on the moving particles is equal and opposite to the force qvh with which the magnetic field acts on the moving particles, so that, disregarding sign, we have

or

$$
q e=q v h
$$

$$
v=\frac{e}{h}
$$

that is to say, the velocity of the particles is equal to the ratio of the electric field intensity $e$ in abvolts per centimeter to the magnetic field intensity $h$ in gausses, on the condition that the fields together produce no deflection of the moving particles.

"Electrochemical equivalent" of $\alpha$ - and $\beta$-particles.-According to the dissociation theory of electrolysis each atom of hydrogen, for example, in a dilute solution of sulphuric acid is isolated and carries a definite amount of charge, and the ratio $\mathrm{m} / \mathrm{q}$ is called the "electrochemical equivalent" of hydrogen, where $m$ is the mass of a hydrogen atom (ion) and $q$ is the charge upon it. The ratio $(\mathrm{m} / \mathrm{q})$ of the mass of a gas ion to the charge upon the ion is called the "electrochemical equivalent" of the gas ion. This ratio is determined by equation (3) or (4) when the electric or magnetic deflection of the particle has been observed and when the velocity of the particle is known. The value so determined is given in grams per abcoulomb and it is equal to $5.36 \times 10^{-8}$ grams per abcoulomb for the $\beta$-particles (electrons), from which it follows that the particles have a mass $\mathrm{I} / 800$ as great as the mass of a hydrogen atom if the charge $q$ is the same in both cases.*

* In regard to the equality of charge on mono-valent ions in electrolytes and on gas ions, see Oliver Lodge's Electrons, pages 77-90, where a simple account is given of the work which has been done by J. J. Thomson in the determination of the value of $q$ (or $m$ ). 


\section{INDEX}

Abampere, Definition of, I9

Abohm, Definition of, 20

Absolute electrometer, The, 152

Abvolt, Definition of, 20

Alternating current transformer, Electromotive force relations of, 45

Ampere, Definition of, 19

turn, Definition of, $\mathbf{8 2}$

Arc, The electric, 287

Atomic theory and mechanical theory, 274

of the electric spark, 279

Attraction of parallel charged plates, I5O

Ballistic galvanometer, The, I24

Binnacle, The, 105

Bunched windings and distributed windings, 75

Bushing, Insulating, Condenser type of, I 48 , I90

Cable insulation, graded, I $_{5} 8$

Maximum electrical stress in, I 73

Canal rays and cathode rays, 282

Velocity of, 292

Capacity of a condenser, I 24

of transmission line, I73

Cathode rays and canal rays, 282

Charge, Electric, I2I Velocity of, 292

Concentrated, 155

Units of, 122

Electrostatic unit of, ${ }_{5} 6$

Clock diagram models of moving wave trains, 231

of a standing wave train, 233

Coil, Action of magnetic field upon, 28 Magnetomotive force of, 78

Compass errors, 106-107

The ship's, 104

Concentrated and distributed magnet poles, 2

Conception of electric wave, 25 I

Condenser, Capacity of, 124

Cylindrical, capacity of, I 7 I

energy of, 129

Spherical, capacity of, 170

The, I23

type of insulating bushing, 148
Condenser, type of insulating bushing, I90

Condensers in parallel, 134

in series, 134

Coulomb's law, 4

Crookes tube, 280

Current, Decay of, in a circuit, 56

Growth of, in a circuit, 54

electric, Kinetic energy of, 53

Curve, Travelling, Equation of, 196

Decay of current in a circuit. 56

Dielectric constant. See Inductivity. hysteresis, 159

Inductivity of, 127

strength, 132

The, 123

Differential equation of wave motion, 196

Direct current dynamo, Fundamental action of, $4 \mathrm{I}$

Distortionless line, 226

Distortion of wave, 224

Distributed and concentrated magnet poles, 2

windings and bunched windings, 75

Dynamo, Direct current, Fundamental equation of, $4 \mathrm{I}$

Electrical and mechanical parallels, I93

Electric arc, The, 287

charge and the condenser, I2I

Concentrated, 155

Units of, 122

current, magnetically defined, I8

Mechanical conception of, 252

field between co-axial cylinders, 156

between concentric spheres, I54

Energy and tension of, ${ }_{53}$

Intensity of, I38

Mechanical conception of, 247

Tapering, Magnetic action of, 248

The, 137

flux, Definition of, I4I

images, Method of, 180

oscillations and electric waves, I93

oscillator, The, I95

The mechanical conception of, 258 
Electric arc, potential, Definition of, 165 due to a line doublet, 183 due to line charge, 176 in the neighborhood of a charged sphere, 168

spark in a gas, atomic theory of, 279

wave, Conception of, 25 I pulse, 208

waves and electric oscillations, 193 Essential features of, 207 reflection of, 2 I 2

Electrodynamometer, The Siemen's, 3I

The Weber, 33

Electrometer, The absolute, 152

Electromagnetic action, 245

Electromagnetism and ferromagnetism, I

Electromotive force, Induced, Law of, 37

Self induced, 49

Electron theory, The, 274

Electrons and ions, 276

Electrostatic attraction of charged plates, 150

unit of charge, The, 156

Energy, Flow of, in the electromagnetic field, 253

stream, Mechanical conception of 253

Equipotential surfaces, 166

Farad, Definition of, 126

Ferromagnetism and electromagnetism, I

Field, electric, between co-axial cylinders, 156

between concentric spheres, I 54

Energy and tension of, 153

Intensity of, 138

magnet, Definition of, 4

Magnetic, Mechanical conception of, 246

The electric, 137

Flames, Electric, Conductivity of, 287

Flinders' bar, Ir4

Flow of energy in the electromagnetic field, 253

Fluoscope, The, 286

Flux density in iron, Magnetic, 87

electric, Definition of, $\mathbf{I}_{4} \mathrm{I}$

Magnetic, Definition of, 39

turns, Discussion of, 48

Focusing tube, Crookes, The, 286

Forced oscillation of a transmission line, $24 \mathrm{I}$

Fortesque's method for designing insulating structures, 187
Galvanometer, The ballistic, r24

The tangent, 22

Gauss' method for measuring earth's magnetic field, II

theorem, $\mathrm{I}_{42}$

Geissler tube, The, 280

Gilbert, The, Definition of, $8 \mathrm{I}$

Graded cable insulation, ${ }_{5} 8$

Growth of current in a circuit, 54

Heeling error of ship's compass, II 5

Hertz oscillator, Mechanical conception of, 26I

Hysteresis, Dielectric, I59

loss in iron, 100

Magnetic, 98

Hysteretic coefficient, Values of, 100

Images, Electric, Method of, 180

Impure waves and pure waves, 222

Induced Electromotive force, Law of, 37

Inductance, Definition of, 47

Mutual, Definition of, 64

of transmission line, 60

Induction, Magnetic. See Flux Density.

Inductivities, Table of, 127

Inductivity of a dielectric, 127

Insulating bushing, Condenser type of, I 48

Condenser type of, 190

structures, Design of, 187

Insulator, Best shape of, 147

Intensity of Magnetization, 85

Ionization of a gas by collision, 278

Ions and electrons, 276

Iron, Magnetization of, 75

Kinetic energy of a current in a circuit, 53

Lenz's principle, 37

Line charge, Electric potential due to, 176

doublet, 183

of force, Definition of as a unit of flux, 39

Geometrical definition of, 6

oscillation, Electric, 228

surges, Transmission, 217

Transmission, Forced oscillations of, 241

Inductance of, 60

Load on a telephone line, 226

Lorenz's method for the absolute measurement of resistance, 7 I

Magnet, Behavior of in a magnetic field, 8 
Magnetic Field, 4

pole, Strength of, 3

poles, I

Distributed and Concen-
trated, 2

circuit, Calculations of, 93

definition of electric current, 18

field, action of upon a coil, 28

Action of upon a magnet, 8

in a long coil, 24

Mechanical conception of, 246

near a long wire, 27

Tapering, Electric equation of, 249

flux, Definition of, 39

through iron, 85

induction. See Flux Density.

hysteresis, 98

permeability, 9I

reluctance, Definition of, 95

Magnetism of a ship, 104

Residual, 87

Magnetization curves, 88

Energy of, 96

Intensity of, 85

of iron, 75

Work required for, 96

table, 9r

Magnetizing force in iron, 82

Magnetomotive force, Definition of, 76 of a coil, 78

Units of, 8I

Maxwell's model of the ether, 246

Maxwell, the, Definition of, 39

Mechanical analogue of transformer, 62 of electric oscillator, 258

of energy stream, 253

of Poynting's theorem, 253

of the electric current, 252

of magnetic and electric fields, 245

and electrical parallels, 193

conception of electric field, 247

theory and atomic theory, 274

Mutual inductance, Definition of, 64

Ohm, Definition of, 20

Oscillation of a transmission line, Electric, 228

Oscillations, electric, 193

forced, of a Transmission line $24 \mathrm{I}$

Oscillator, Electric, Mechanical conception of, 258

The Electric, 195

Ozone machine, The, 288

Permeability, Magnetic, 9I

Poles of a magnet, I
Potential, Definition of, 165

due to a line doublet, 183

Electric, Due to a line charge, I 76

values in the neighborhood of a charged sphere, 168

Poynting's theorem, 253

Examples of, 254

Pure waves and impure waves, 222

Quadrantal correctors, II 2

error of ship's compass, 108

Radioactivity, 289

Reluctance, magnetic, Definition of, 95

Residual magnetism, 87

Ribbon rave, The, 209

Roentgen rays, 285

Secohmmeter, The, I35

Self induced electromotive force, 49

Semi-circular correctors, 107

error of compass, 107

Ship's compass, Adjustment of, II6

The, 104

magnetism, 104

permanent magnetism, 106

temporary magnetism, 106

Siemen's Electrodynamometer, The, 3 I

Simple mode of oscillation of transmission line, 234

Specific capacity of a dielectric. See Inductivity.

inductive capacity. See Inductivity.

Standing wave trains, clock diagram models of, 233

waves, Experimental demonstration of, 244

Strength of a dielectric, 132

of pole, Definition of, 3

Superposition, Principle of, 2 I I

Surges on a transmission line, 2 I 7

Tangent galvanometer, The, $\mathbf{2 2}$

Tapering electric field, Magnetic action of, 248

magnetic field, Electric action of, 249

Transformer, Alternating current, Electromotive force relations of, 45

Mechanical analogue of, 62

Transmission line, Capacity of, I 73 Electric oscillation of, 228 Forced oscillations of, 24I

Inductance of, 60

Maximum electrical stress between the wires of, I75 
Transmission line, Simple mode of oscillation of, 234

surges, 217

Wave motion on, 202

Wave train on, 209

Travelling curve, Equation of, I96

Unit pole, Definition of, 2

Volt, Definition of, 20

Wave distortion, 224 motion, Differential equation of, 196
Wave motion on a transmission line, 202

The ribbon, 209

train, Electric, 209

trains, Moving, Clock diagram model of, 23I

Standing, Clock diagram models of, 233

Waves, Electric, I93

Reflection of, 212

pure and impure, 222

Standing, Experimental Demonstration of, 244

Superposition of, 2 II

Weber Electrodynamometer, The, 33

Windings, Distributed and bunched, 75 








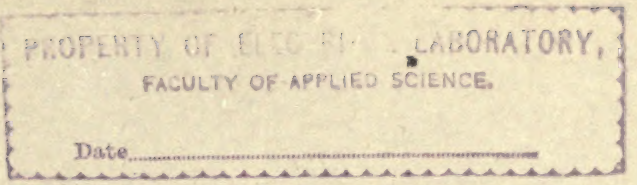

\section{PLEASE DO NOT REMOVE CARDS OR SLIPS FROM THIS POCKET}

\section{UNIVERSITY OF TORONTO LIBRARY}

S\&M

A

127 
